\title{
Copper-Free Sandmeyer-Type Reaction for the Synthesis of Sulfonyl Fluorides
}

\author{
Tao Zhong, Meng-Ke Pang, Zhi-Da Chen, Bin Zhang, Jiang Weng, ${ }^{*}$ and Gui Lu \\ Guangdong Provincial Key Laboratory of Chiral Molecule and Drug Discovery, School of \\ Pharmaceutical Sciences, Sun Yat-sen University, Guangzhou, 510006, (P.R. China). \\ E-mail: wengj2@mail.sysu.edu.cn
}

\section{Table of Contents}

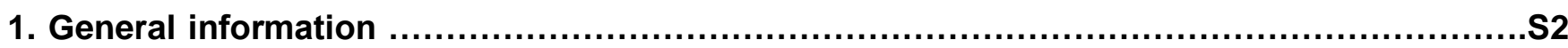

2. Typical procedure for the synthesis of aryldiazonium salts.................................... 2

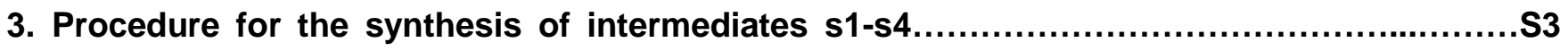

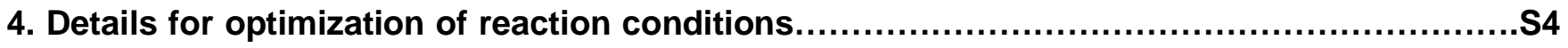

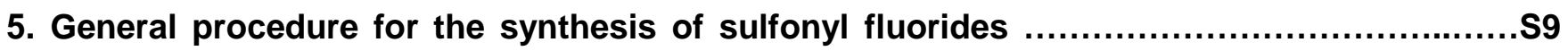

6. General procedure for the one-pot synthesis of sulfonyl fluorides............................ 9

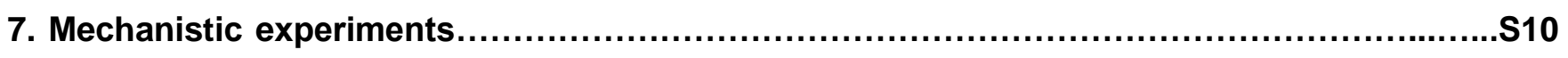

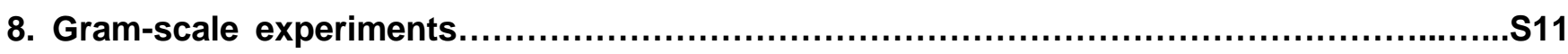

9. Characterization data of compounds 2a-2z, 2aa-2ai, 2ak, 3aj and s1-s4.....................S12

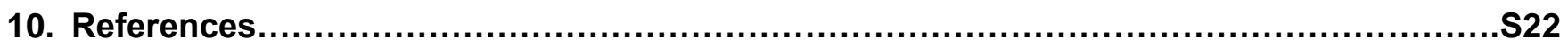

11. ${ }^{1} \mathrm{H},{ }^{13} \mathrm{C}$ and ${ }^{19} \mathrm{~F}$ NMR Spectra of Compounds 2a-2z, 2aa-2ai, 3aj and 3aj s1-s4................S23 


\section{General Information}

All the commercial reagents were used as such without further purification. All solvents were used as commercial anhydrous grade without further purification. The flash column chromatography was carried out over silica gel $(230-400 \mathrm{mesh}) .{ }^{1} \mathrm{H},{ }^{13} \mathrm{C}$ and ${ }^{19} \mathrm{~F}$ NMR spectra were recorded on a Bruker Avance- $300 \mathrm{MHz}$ spectrometer or Bruker Avance- $400 \mathrm{MHz}$ spectrometer or Bruker Avance$500 \mathrm{MHz}$ spectrometer. Chemical shifts in ${ }^{1} \mathrm{H}$ NMR spectra were reported in parts per million (ppm, $\delta$ ) downfield from the internal standard $\mathrm{Me}_{4} \mathrm{Si}$ (TMS, $\delta=0 \mathrm{ppm}$ ). Chemical shifts in ${ }^{13} \mathrm{C}$ NMR spectra were reported relative to the central line of the chloroform signal $(\delta=77.0 \mathrm{ppm})$. Peaks were labeled as singlet $(\mathrm{s})$, doublet $(\mathrm{d})$, triplet $(\mathrm{t})$, quartet $(\mathrm{q})$, and multiplet $(\mathrm{m})$. Melting points were measured on OptiMelt automated melting point system apparatus and uncorrected. The ionization method of the HRMS was electron ionization (EI) or electrospray ionization (ESI), and the mass analyzer type was TOF for ESI and Double focusing mass analyzer for El. The $\mathrm{m} / \mathrm{z}$ ratios are reported in Daltons; high resolution values are calculated to four decimal places from the molecular formula Every reaction should be performed behind a safety shield. Chemical yields refer to pure isolated substances.

\section{Typical procedure for the synthesis of aryldiazonium salts ${ }^{1}$}

Method A: In a $25 \mathrm{~mL}$ round-bottom flask, the arylamine $(5 \mathrm{mmol})$ was dissolved in a mixture of ethanol $(1.5 \mathrm{~mL})$ and $\mathrm{HBF}_{4}(\mathrm{aq} .48 \%$, $1.25 \mathrm{~mL})$. Then tert-butyl nitrite $(1.35 \mathrm{~mL})$ was added dropwise to the solution at $0{ }^{\circ} \mathrm{C}$. The mixture was stirred at room temperature for $1 \mathrm{~h}$ before diethyl ether $(5 \mathrm{~mL})$ was added to precipitate the arenediazonium tetrafluoroborate. (Note: If there is no solid formd after adding diethyl ether, put the flask in the refrigerator overnight). The solid was filtered off and washed with diethyl ether $(3 \times 5$ $\mathrm{mL}$ ). The arenediazonium tetrafluoroborate was dried in vacuo for 25 minutes and was then directly used without further purification (Scheme S1).

Scheme S1. Preparation of aryldiazonium tetrafluoroborates (Method A)

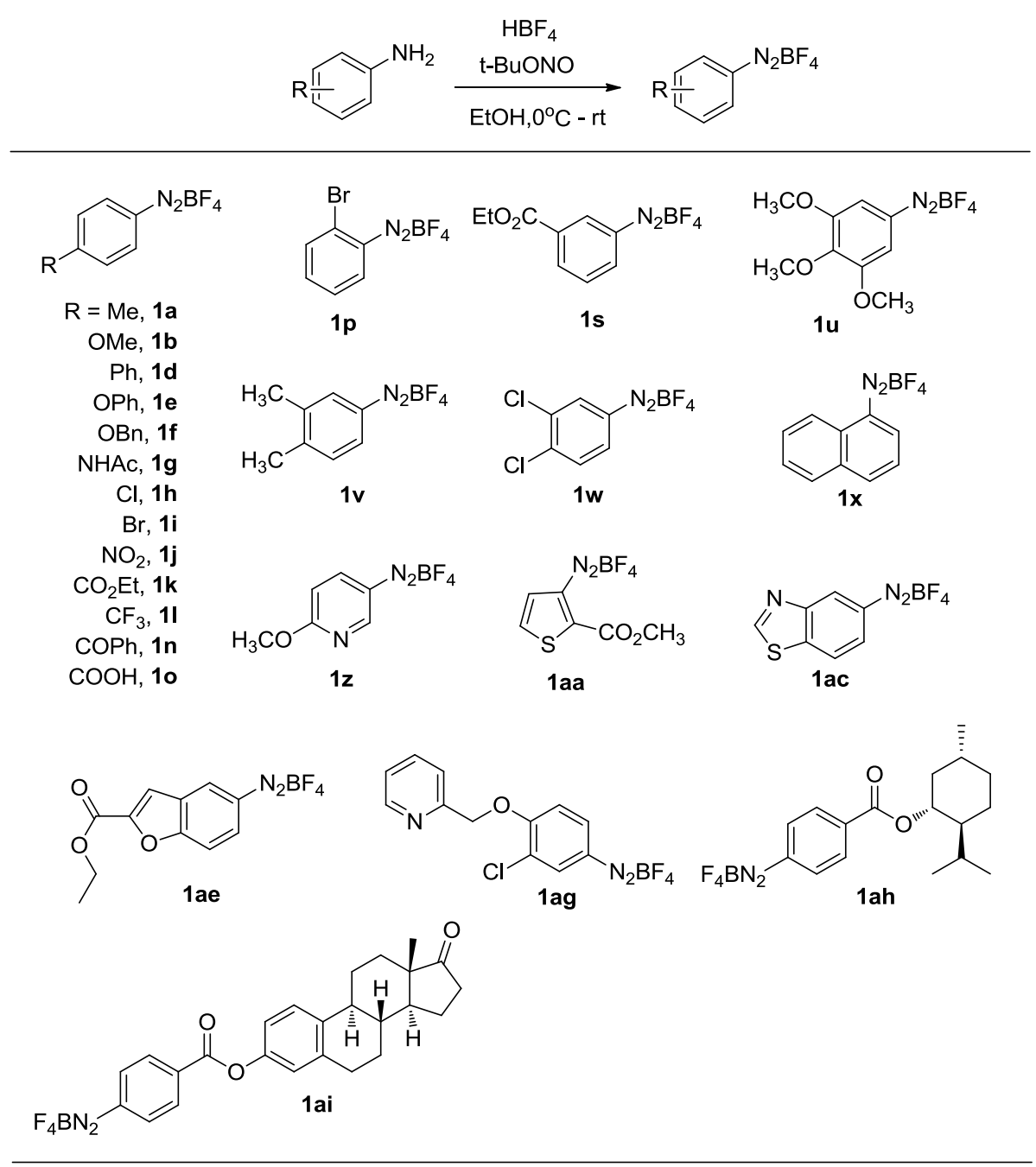

Method B: In a $25 \mathrm{~mL}$ round-bottom flask, the arylamine $(5 \mathrm{mmol})$ was dissolved in a mixture of $\mathrm{H}_{2} \mathrm{O}(2.5 \mathrm{~mL})$ and $\mathrm{HBF}_{4}(\mathrm{aq} .48 \%$, $1.5 \mathrm{~mL})$. After stirring for 15 minutes, the solution of $\mathrm{NaNO}_{2}\left(379.5 \mathrm{mg}, 1.1\right.$ equiv, in $\left.1 \mathrm{~mL} \mathrm{H}_{2} \mathrm{O}\right)$ was added dropwise at $0{ }^{\circ} \mathrm{C}$. The mixture was stirred for another $30 \mathrm{~min}$ at $0{ }^{\circ} \mathrm{C}$. Then, the arenediazonium tetrafluoroborate was removed by filtration and washed 
successively twice with diethyl ether $(5 \mathrm{~mL})$. The crude product was dried in vacuo for 25 min and was then directly used without further purification (Scheme S2).

Scheme S2. Preparation of aryldiazonium salts (Method B)<smiles>CC(C)Nc1ccc(N)cc1</smiles>

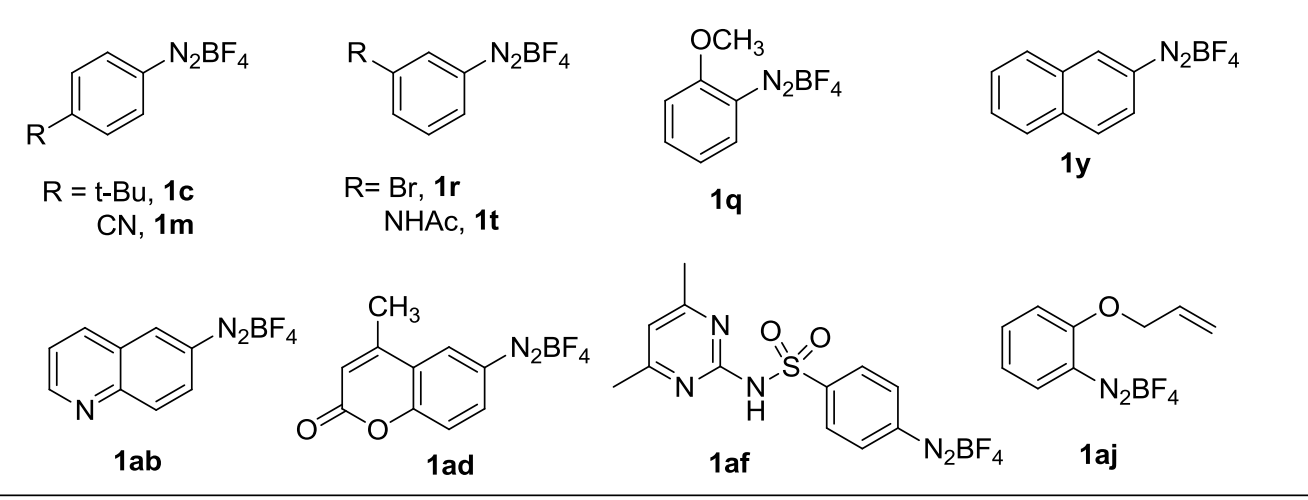

\section{Procedure for the Synthesis of Intermediates $s 1^{2}, s 2^{3}, s 3, s 4^{4}$}

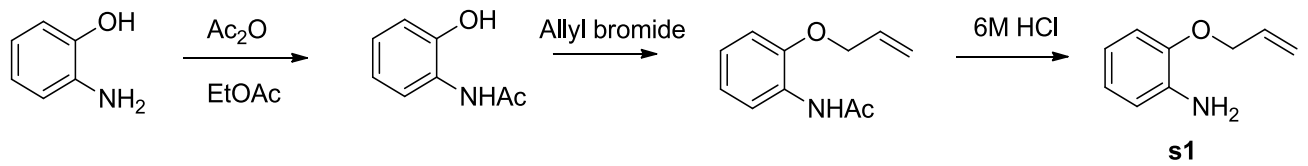

To a solution of 2-aminophenol $(4.06 \mathrm{~g}, 37.2 \mathrm{mmol})$ in EtOAc $(40 \mathrm{~mL})$ was added dropwise $\mathrm{Ac}_{2} \mathrm{O}(8.2 \mathrm{~mL}, 84 \mathrm{mmol})$ at $0{ }^{\circ} \mathrm{C}$, and then the mixture was stirred at room temperature for $4 \mathrm{~h}$. The mixture was concentrated in vacuo, and the residual solid was recrystallized from $\mathrm{MeOH}$ /acetone (1:1) and $\mathrm{CH}_{2} \mathrm{Cl}_{2}$ at $0{ }^{\circ} \mathrm{C}$. 2-Hydroxyacetanilide $(4.73 \mathrm{~g}, 31.3 \mathrm{mmol}, 84 \%)$ was isolated as a light yellow powder.

To a solution of 2-hydroxyacetanilide $(2.92 \mathrm{~g}, 19.3 \mathrm{mmol})$ and $\mathrm{K}_{2} \mathrm{CO}_{3}(42.67 \mathrm{~g}, 19.3 \mathrm{mmol})$ in anhydrous acetone $(7 \mathrm{~mL})$ was added dropwise allyl bromide $\left(1.67 \mathrm{~mL}, 19.3 \mathrm{mmol}\right.$ ) at $0{ }^{\circ} \mathrm{C}$. The mixture was warmed to reflux (oil bath) and stirred for $20 \mathrm{~h}$. The organic phase was extracted with $\mathrm{Et}_{2} \mathrm{O}(2 \times 50 \mathrm{~mL})$, washed with water $(50 \mathrm{~mL})$, with a solution of $\mathrm{NaOH}(10 \%)$ and dried over anhydrous $\mathrm{Na}_{2} \mathrm{SO}_{4}$. The crude product was purified by flash column chromatography (petroleum ether/ ethyl acetate 10:1). $\mathrm{N}$-(2-(allyloxy) phenyl) acetamide was isolated in $96 \%$ yield.

A solution of $\mathrm{N}-(2$-(allyloxy) phenyl) acetamide $(3.55 \mathrm{~g}, 18.57 \mathrm{mmol})$ in $6 \mathrm{M} \mathrm{HCl}(4.46 \mathrm{~mL})$ was refluxed (oil bath) for $4 \mathrm{~h}$. The mixture was basified with $4 \mathrm{~N}$ sodium hydroxide solution until $\mathrm{pH}=14$. This basified solution was extracted with EtOAc $(2 \times 20 \mathrm{~mL})$ and dried over anhydrous $\mathrm{Na}_{2} \mathrm{SO}_{4}$. The organic solvent was evaporated and the crude product was purified by flash column chromatography to give the desired product $\mathbf{s} 1$ (95\% yield) as orange oil.<smiles>O=C(Cl)c1ccc([N+](=O)[O-])cc1</smiles><smiles>CC(C)C1CC[C@@H](C)CC1O</smiles>

D-Menthol<smiles>CC(C)C1CC[C@@H](C)CC1OC(=O)c1ccc([N+](=O)[O-])cc1</smiles>

$4,86 \%$<smiles>CC(C)C1CC[C@H](C)CC1OC(=O)c1ccc(N)cc1</smiles>

Under nitrogen atmosphere, compound $3(1.8 \mathrm{~g}, 9.7 \mathrm{mmol})$ was dissolved in dry pyridine $(20 \mathrm{~mL})$, then D-menthol $(1.5 \mathrm{~g}, 9.7 \mathrm{mmol})$ was added in one portion and the resulting mixture was stirred at room temperature for $16 \mathrm{~h}$, after removal of pyridine under reduced pressure, the residue was dissolved in dichloromethane $(30 \mathrm{~mL})$ and washed successively with $1 \mathrm{M} \mathrm{HCl}$, saturated $\mathrm{NaHCO}_{3}$ aqueous solution and brine, the separated organic layer was dried over anhydrous $\mathrm{Na}_{2} \mathrm{SO}_{4}$ and concentrated under reduced pressure, the residue was purified by column chromatography (silica gel, 10:1 petroleum ether to ethyl acetate, $\mathrm{v} / \mathrm{v}$ ) to afford the desired compound 4 as a yellow solid $(2.40 \mathrm{~g}, 86 \%$ yield). 
To the methanol $(20 \mathrm{~mL})$ solution of $4(2.555 \mathrm{~g}), 10 \%$ palladium on active carbon $(202 \mathrm{mg})$ was added under nitrogen atmosphere. Hydrogen gas was bubbled into the suspension with stirring at ambient temperature for $15 \mathrm{~h}$. After the palladium on carbon was removed by filtration, the solvent was removed under reduced pressure, to afford pale orange syrup s2 (2.23 g, 96\%).

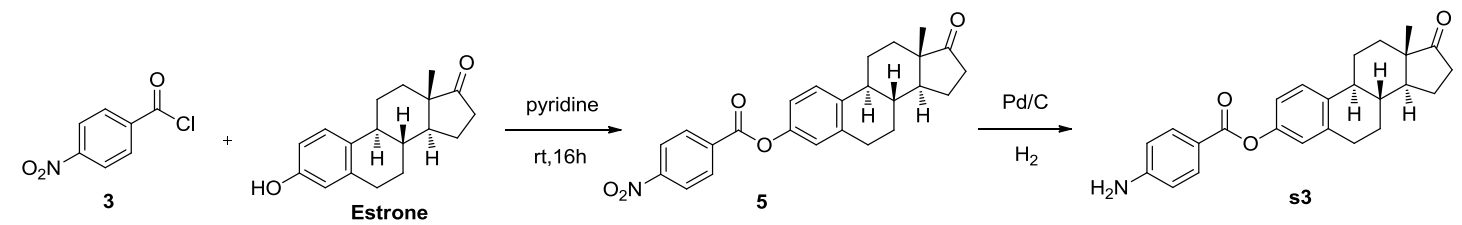

s3 is synthesized according to the same procedures as s2 by using Estrone (1,3,5(10)-Estratrien-3-ol-17-one).

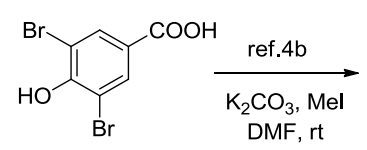<smiles>COc1c(Br)cc(C(=O)O[Na])cc1Br</smiles><smiles>COc1c(Br)cc(C(=O)NN)cc1Br</smiles><smiles>Nc1ccc(C(=O)O)cc1</smiles><smiles>COc1c(Br)cc(-c2nnc(-c3ccc(N)cc3)o2)cc1Br</smiles>

s4 was synthesized according to the literature method. ${ }^{4}$

\section{Details for optimization of reaction conditions}

Table S1. Evaluation of solvents (DABSO as the $\mathrm{SO}_{2}$ surrogate)

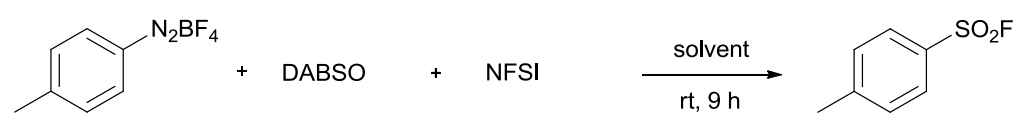

$1 \mathrm{a}$

$2 \mathbf{a}$

\begin{tabular}{|c|c|c|c|c|}
\hline Entry $^{\text {[a] }}$ & $\mathrm{SO}_{2}$ surrogate & F source & Solvent & Yield (\%) ${ }^{[b]}$ \\
\hline 1 & DABSO $(0.6$ eq) & NFSI (1.2 eq) & $\mathrm{CH}_{3} \mathrm{CN}$ & $N D^{[c]}$ \\
\hline 2 & DABSO (0.6 eq) & NFSI (1.2 eq) & DMSO & ND \\
\hline 3 & DABSO $(0.6 \mathrm{eq})$ & NFSI (1.2 eq) & $\mathrm{PhCH}_{3}$ & trace \\
\hline 4 & DABSO (0.6 eq) & NFSI (1.2 eq) & THF & trace \\
\hline 5 & DABSO (0.6 eq) & $\mathrm{NFSI}$ (1.2eq) & Acetone & ND \\
\hline 6 & DABSO (0.6 eq) & NFSI (1.2 eq) & 1,4-dioxane & ND \\
\hline 7 & DABSO (0.6 eq) & NFSI (1.2 eq) & DCM & 10 \\
\hline 8 & DABSO (0.6 eq) & $\mathrm{NFSI}(1.2 \mathrm{eq})$ & $\mathrm{MeOH}$ & 5 \\
\hline 9 & DABSO ( $0.6 \mathrm{eq})$ & NFSI (1.2 eq) & $\mathrm{CHCl}_{3}$ & 13.5 \\
\hline
\end{tabular}

[a] The reaction conditions: $1 \mathrm{a}(0.2 \mathrm{mmol}, 1.0 \mathrm{eq}), \mathrm{SO}_{2}$ surrogate, $\mathrm{F}$ source, solvent $(2 \mathrm{~mL}), \mathrm{T}, \mathrm{N}_{2}, 9 \mathrm{~h}$;

[b] ${ }^{19} \mathrm{~F}$ NMR yield calculated with $\mathrm{PhCF}_{3}$ as internal standard;

[c] $p$-acetotoluidide as the product in $60 \%$ yield. 
Table S2. Evaluation of fluorine sources and additives (DABSO as the $\mathrm{SO}_{2}$ surrogate)

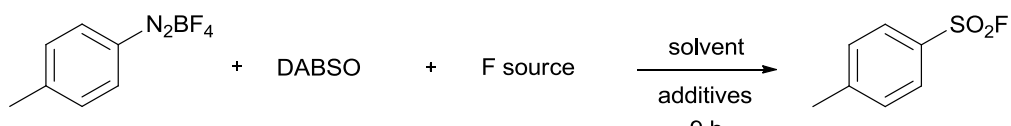

$9 \mathrm{~h} 2 \mathrm{a}$

\begin{tabular}{|c|c|c|c|c|c|c|}
\hline Entry $^{\text {[a] }}$ & $\mathrm{SO}_{2}$ surrogate & F source & Solvent & $\mathbf{T}\left({ }^{\circ} \mathbf{C}\right)$ & $\begin{array}{l}\text { Additive } \\
\text { (10 mol\%) }\end{array}$ & Yield (\%) ${ }^{[\mathrm{DT}}$ \\
\hline 1 & DABSO (0.6 eq) & KFHF & $\mathrm{CHCl}_{3}$ & $\mathrm{rt}$ & - & ND \\
\hline 2 & DABSO (0.6 eq) & TBAF & $\mathrm{CHCl}_{3}$ & $r \mathrm{t}$ & - & ND \\
\hline 3 & DABSO (0.6 eq) & $\mathrm{AgF}$ & $\mathrm{CHCl}_{3}$ & $\mathrm{rt}$ & - & ND \\
\hline 4 & DABSO (0.6 eq) & NFSI (1.2 eq) & $\mathrm{CH}_{3} \mathrm{CN}$ & $\mathrm{rt}$ & $\mathrm{CuBr}_{2}$ & $<5$ \\
\hline 5 & DABSO (0.6 eq) & NFSI (1.2 eq) & $\mathrm{CH}_{3} \mathrm{CN}$ & $\mathrm{rt}$ & Cul & $<5$ \\
\hline 6 & DABSO (0.6 eq) & NFSI (1.2 eq) & $\mathrm{CH}_{3} \mathrm{CN}$ & 80 & $\mathrm{CuBr}_{2}$ & $<5$ \\
\hline 7 & DABSO (0.6 eq) & NFSI (1.2 eq) & $\mathrm{CH}_{3} \mathrm{CN}$ & 80 & Cul & $<5$ \\
\hline 8 & DABSO (0.6 eq) & NFSI (1.2 eq) & DCE & 60 & $\mathrm{Cu}(\mathrm{OAc})_{2}$ & ND \\
\hline 9 & DABSO (0.6 eq) & NFSI (1.2 eq) & DCE & 60 & $\mathrm{Cu}(\mathrm{acac})_{2}$ & ND \\
\hline
\end{tabular}

[a] The reaction conditions: 1a $(0.2 \mathrm{mmol}, 1.0 \mathrm{eq}), \mathrm{SO}_{2}$ surrogate, $\mathrm{F}$ source, solvent $(2 \mathrm{~mL}), \mathrm{T}, \mathrm{N}_{2}, 9 \mathrm{~h}$;

[b] ${ }^{19} \mathrm{~F}$ NMR yield calculated with $\mathrm{PhCF}_{3}$ as internal standard.

Table S3. Evaluation of solvents (Rongalite as the $\mathrm{SO}_{2}$ surrogate)

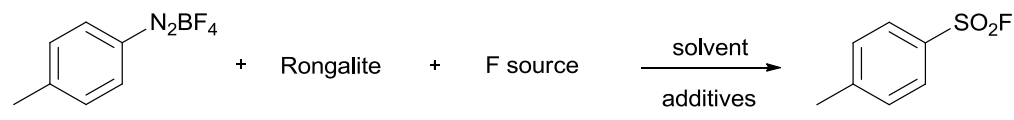

$1 a$

$9 \mathrm{~h}$

\begin{tabular}{|c|c|c|c|c|c|c|}
\hline Entry $^{[\text {a] }}$ & $\mathrm{SO}_{2}$ surrogate & F source & Solvent & $\mathrm{T}\left({ }^{\circ} \mathrm{C}\right)$ & $\begin{array}{c}\text { Additive } \\
\text { (10 mol\%) }\end{array}$ & Yield (\%) ${ }^{[\mathrm{b}]}$ \\
\hline 1 & Rongalite (1.2 eq) & NFSI (1.2 eq) & DMSO & rt & - & ND \\
\hline 2 & Rongalite (1.2 eq) & NFSI (1.2 eq) & $\mathrm{DCM}$ & rt & - & 10 \\
\hline 3 & Rongalite (1.2 eq) & Selectfluor (1.2 eq) & DCM & rt & - & ND \\
\hline 4 & Rongalite (1.2 eq) & Selectfluor (1.2 eq) & DMF & 60 & - & 15 \\
\hline 5 & Rongalite (1.2 eq) & Selectfluor (1.2 eq) & $\mathrm{MeOH}$ & 60 & - & 28 \\
\hline 6 & Rongalite (1.2 eq) & Selectfluor (1.2 eq) & $\mathrm{EtOH}$ & 60 & - & 10 \\
\hline 7 & Rongalite (1.2 eq) & Selectfluor (1.2 eq) & $\mathrm{MeOH}$ & 60 & TBAB & ND \\
\hline 8 & Rongalite (1.2 eq) & Selectfluor (1.2 eq) & $\mathrm{DCM}$ & 60 & TBAB & ND \\
\hline 9 & Rongalite (1.2 eq) & Selectfluor (1.2 eq) & DMF & 60 & TBAB & ND \\
\hline
\end{tabular}

[a] The reaction conditions: $1 \mathrm{a}(0.2 \mathrm{mmol}, 1.0 \mathrm{eq}), \mathrm{SO}_{2}$ surrogate, $\mathrm{F}$ source, solvent $(2 \mathrm{~mL}), \mathrm{T}, \mathrm{N}_{2}, 9 \mathrm{~h}$;

$[\mathrm{b}]{ }^{19} \mathrm{~F}$ NMR yield calculated with $\mathrm{PhCF}_{3}$ as internal standard 
Table S4. Optimization of reaction conditions $\left(\mathrm{Na}_{2} \mathrm{~S}_{2} \mathrm{O}_{5}\right.$ as the $\mathrm{SO}_{2}$ surrogate)

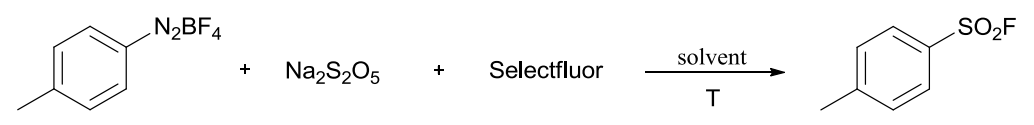

$1 \mathrm{a}$

2

\begin{tabular}{|c|c|c|c|c|c|c|}
\hline Entry $^{\text {[a] }}$ & $\mathrm{SO}_{2}$ surrogate & F source & Solvent & $\mathrm{T}\left({ }^{\circ} \mathrm{C}\right)$ & Time & Yield (\%) ${ }^{[D]}$ \\
\hline 1 & $\mathrm{Na}_{2} \mathrm{~S}_{2} \mathrm{O}_{5}(1.2 \mathrm{eq})$ & Selectfluor (1.2 eq) & DMF & $\mathrm{rt}$ & $9 \mathrm{~h}$ & 10 \\
\hline 2 & $\mathrm{Na}_{2} \mathrm{~S}_{2} \mathrm{O}_{5}(1.2 \mathrm{eq})$ & Selectfluor (1.2 eq) & DMF & 60 & $9 \mathrm{~h}$ & 12 \\
\hline 3 & $\mathrm{Na}_{2} \mathrm{~S}_{2} \mathrm{O}_{5}(1.2 \mathrm{eq})$ & Selectfluor (1.2 eq) & $\mathrm{MeOH}$ & $\mathrm{rt}$ & $9 \mathrm{~h}$ & 23 \\
\hline 4 & $\mathrm{Na}_{2} \mathrm{~S}_{2} \mathrm{O}_{5}(1.2 \mathrm{eq})$ & Selectfluor (1.2 eq) & $\mathrm{MeOH}$ & 60 & $9 \mathrm{~h}$ & 32 \\
\hline 5 & $\mathrm{Na}_{2} \mathrm{~S}_{2} \mathrm{O}_{5}(2 \mathrm{eq})$ & Selectfluor (2 eq) & $\mathrm{MeOH}$ & 60 & $9 \mathrm{~h}$ & 46 \\
\hline 6 & $\mathrm{Na}_{2} \mathrm{~S}_{2} \mathrm{O}_{5}(1.5 \mathrm{eq})$ & Selectfluor (3 eq) & $\mathrm{MeOH}$ & 60 & $9 \mathrm{~h}$ & 20 \\
\hline 7 & $\mathrm{Na}_{2} \mathrm{~S}_{2} \mathrm{O}_{5}(3 \mathrm{eq})$ & Selectfluor (2 eq) & $\mathrm{MeOH}$ & 60 & $9 \mathrm{~h}$ & 58 \\
\hline 8 & $\mathrm{Na}_{2} \mathrm{~S}_{2} \mathrm{O}_{5}(3 \mathrm{eq})$ & Selectfluor (2 eq) & $\mathrm{MeOH}$ & 60 & $24 \mathrm{~h}$ & 32 \\
\hline 9 & $\mathrm{Na}_{2} \mathrm{~S}_{2} \mathrm{O}_{5}(3 \mathrm{eq})$ & Selectfluor (2 eq) & $\mathrm{MeOH}$ & 60 & $6 \mathrm{~h}$ & 56 \\
\hline 10 & $\mathrm{Na}_{2} \mathrm{~S}_{2} \mathrm{O}_{5}(4 \mathrm{eq})$ & Selectfluor (2 eq) & $\mathrm{MeOH}$ & 60 & $9 \mathrm{~h}$ & 42 \\
\hline 11 & $\mathrm{Na}_{2} \mathrm{~S}_{2} \mathrm{O}_{5}(3 \mathrm{eq})$ & Selectfluor (3 eq) & $\mathrm{MeOH}$ & 60 & $9 \mathrm{~h}$ & 44 \\
\hline 12 & $\mathrm{Na}_{2} \mathrm{~S}_{2} \mathrm{O}_{5}(3 \mathrm{eq})$ & Selectfluor (2 eq) & $\mathrm{MeOH}$ & 50 & $9 \mathrm{~h}$ & 55 \\
\hline 13 & $\mathrm{Na}_{2} \mathrm{~S}_{2} \mathrm{O}_{5}(3 \mathrm{eq})$ & Selectfluor (2 eq) & $\mathrm{MeOH}$ & 70 & $9 \mathrm{~h}$ & 63 \\
\hline 14 & $\mathrm{Na}_{2} \mathrm{~S}_{2} \mathrm{O}_{5}(3 \mathrm{eq})$ & Selectfluor (2 eq) & $\mathrm{MeOH}$ & 80 & $9 \mathrm{~h}$ & 62 \\
\hline $15^{[c]}$ & $\mathrm{Na}_{2} \mathrm{~S}_{2} \mathrm{O}_{5}(3 \mathrm{eq})$ & Selectfluor (2 eq) & $\mathrm{MeOH}$ & 70 & $9 \mathrm{~h}$ & $65^{[\mathrm{d}]}$ \\
\hline $16^{[\mathrm{e}]}$ & $\mathrm{Na}_{2} \mathrm{~S}_{2} \mathrm{O}_{5}(3 \mathrm{eq})$ & Selectfluor (2 eq) & $\mathrm{MeOH}$ & 70 & $9 \mathrm{~h}$ & 32 \\
\hline
\end{tabular}

[a] The reaction conditions: $1 \mathrm{a}(0.2 \mathrm{mmol}, 1.0 \mathrm{eq}), \mathrm{SO}_{2}$ surrogate, $\mathrm{F}$ source, solvent $(2 \mathrm{~mL}), \mathrm{T}, \mathrm{N}_{2}, 9 \mathrm{~h}$;

[b: ${ }^{19} \mathrm{~F}$ NMR yield calculated with $\mathrm{PhCF}_{3}$ as internal standard;

[c] In $1 \mathrm{~mL} \mathrm{MeOH;}$

[d] $66 \%$ isolated yield;

[e] Without $\mathrm{N}_{2}$ protection. 
Table S5. Selected optimization experiments

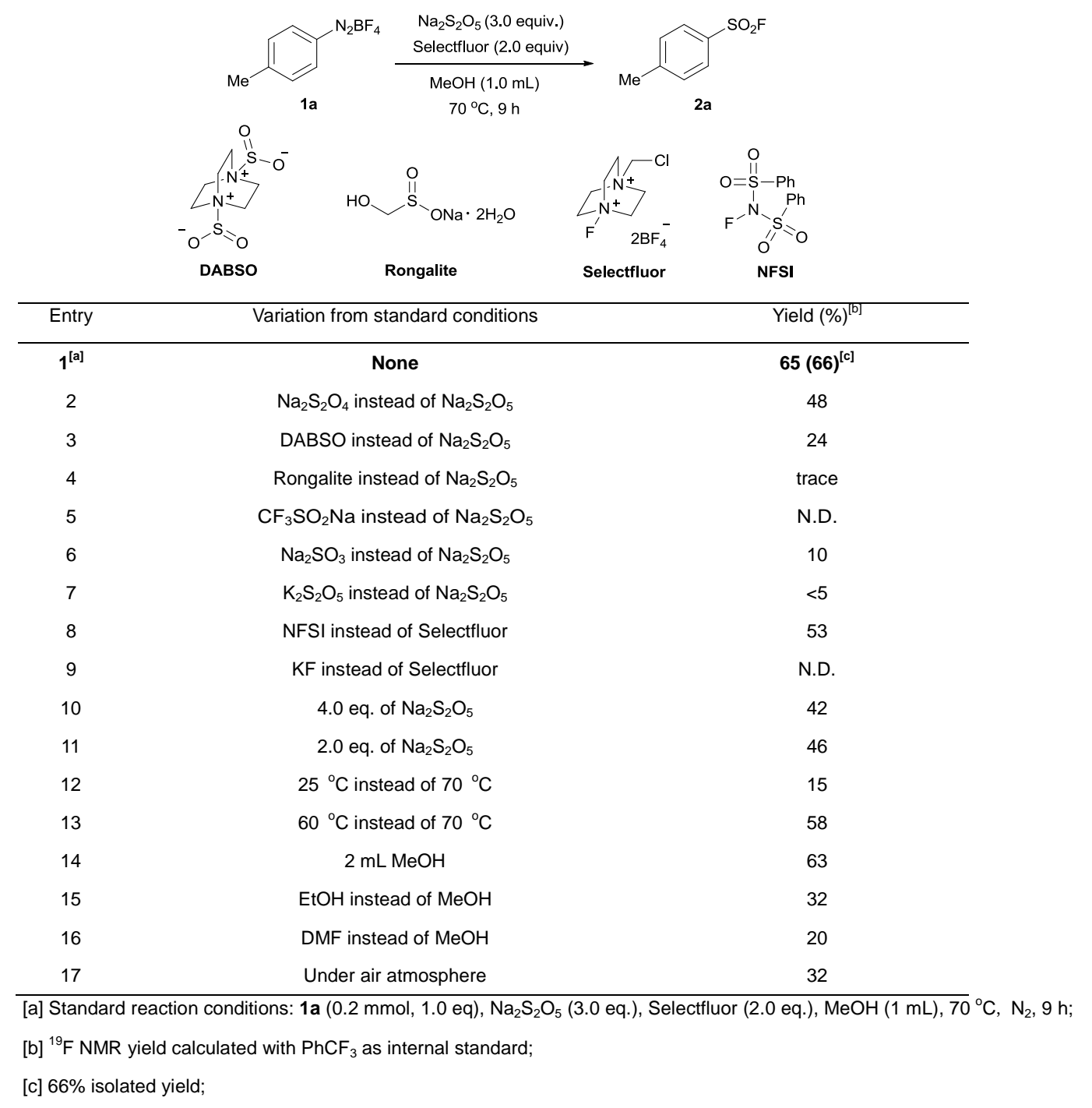


Table S6. Reevaluation of the reaction conditions using $1 \mathrm{j}$ as the model substrate

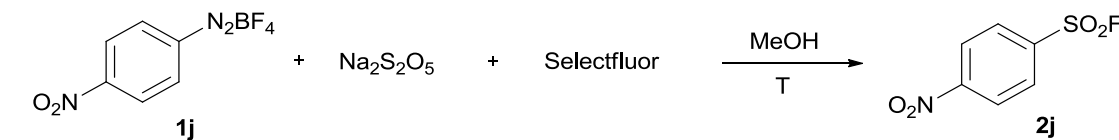

\begin{tabular}{|c|c|c|c|c|c|}
\hline Entry $^{[\text {a] }}$ & $\mathrm{SO}_{2}$ surrogate & F source & Solvent & $\mathrm{T}\left({ }^{\circ} \mathrm{C}\right)$ & Yield (\%) ${ }^{[D]}$ \\
\hline 1 & $\mathrm{Na}_{2} \mathrm{~S}_{2} \mathrm{O}_{5}(1.2 \mathrm{eq})$ & Selectfluor (1.2 eq) & $\mathrm{MeOH}$ & 70 & 41 \\
\hline 2 & $\mathrm{Na}_{2} \mathrm{~S}_{2} \mathrm{O}_{5}(3 \mathrm{eq})$ & Selectfluor (2 eq) & МeOH & 70 & $34^{[c]}$ \\
\hline 3 & $\mathrm{Na}_{2} \mathrm{~S}_{2} \mathrm{O}_{5}(1.2 \mathrm{eq})$ & Selectfluor (1.5 eq) & $\mathrm{MeOH}$ & 70 & 40 \\
\hline 4 & $\mathrm{Na}_{2} \mathrm{~S}_{2} \mathrm{O}_{5}(1.2 \mathrm{eq})$ & Selectfluor (2 eq) & $\mathrm{MeOH}$ & 70 & 43 \\
\hline 5 & $\mathrm{Na}_{2} \mathrm{~S}_{2} \mathrm{O}_{5}(1.5 \mathrm{eq})$ & Selectfluor (1.2 eq) & $\mathrm{MeOH}$ & 70 & trace \\
\hline 6 & $\mathrm{Na}_{2} \mathrm{~S}_{2} \mathrm{O}_{5}(1.5 \mathrm{eq})$ & Selectfluor (1.5 eq) & $\mathrm{MeOH}$ & 70 & 40 \\
\hline 7 & $\mathrm{Na}_{2} \mathrm{~S}_{2} \mathrm{O}_{5}(1.5 \mathrm{eq})$ & Selectfluor (2 eq) & $\mathrm{MeOH}$ & 70 & 60 \\
\hline 8 & $\mathrm{Na}_{2} \mathrm{~S}_{2} \mathrm{O}_{5}(2 \mathrm{eq})$ & Selectfluor (1.2 eq) & $\mathrm{MeOH}$ & 70 & trace \\
\hline 9 & $\mathrm{Na}_{2} \mathrm{~S}_{2} \mathrm{O}_{5}(2 \mathrm{eq})$ & Selectfluor (1.5 eq) & $\mathrm{MeOH}$ & 70 & trace \\
\hline 10 & $\mathrm{Na}_{2} \mathrm{~S}_{2} \mathrm{O}_{5}(2 \mathrm{eq})$ & Selectfluor (2 eq) & $\mathrm{MeOH}$ & 70 & 37 \\
\hline 11 & $\mathrm{Na}_{2} \mathrm{~S}_{2} \mathrm{O}_{5}(1.1 \mathrm{eq})$ & Selectfluor (1.6 eq) & $\mathrm{MeOH}$ & 70 & 38 \\
\hline 12 & $\mathrm{Na}_{2} \mathrm{~S}_{2} \mathrm{O}_{5}(1.1 \mathrm{eq})$ & Selectfluor (2 eq) & $\mathrm{MeOH}$ & 70 & 40 \\
\hline 13 & $\mathrm{Na}_{2} \mathrm{~S}_{2} \mathrm{O}_{5}(1.5 \mathrm{eq})$ & Selectfluor (2 eq) & $\mathrm{MeOH}$ & 50 & 48 \\
\hline 14 & $\mathrm{Na}_{2} \mathrm{~S}_{2} \mathrm{O}_{5}(1.5 \mathrm{eq})$ & Selectfluor (2 eq) & $\mathrm{MeOH}$ & 80 & 54 \\
\hline 15 & $\mathrm{Na}_{2} \mathrm{~S}_{2} \mathrm{O}_{5}(1.5 \mathrm{eq})$ & Selectfluor (2 eq) & $\mathrm{MeOH}$ & 90 & 54 \\
\hline 16 & $\mathrm{Na}_{2} \mathrm{~S}_{2} \mathrm{O}_{5}(1.5 \mathrm{eq})$ & Selectfluor (2 eq) & $\mathrm{MeOH}^{[\mathrm{d}]}$ & 70 & 54 \\
\hline
\end{tabular}

[a] The reaction conditions: $\mathbf{1 j}(0.2 \mathrm{mmol}, 1.0 \mathrm{eq}), \mathrm{Na}_{2} \mathrm{~S}_{2} \mathrm{O}_{5}$, Selectfluor, $\mathrm{MeOH}(1 \mathrm{~mL}), \mathrm{T}, \mathrm{N}_{2}, 9 \mathrm{~h}$;

[b] ${ }^{19} \mathrm{~F}$ NMR yield calculated with $\mathrm{PhCF}_{3}$ as internal standard;

[c] nitrobenzene as the byproduct in $45 \%$ yield;

[d] absolute anhydrous $\mathrm{MeOH}$ was used.

Scheme S3. Unsuccessful and unsatisfactory results

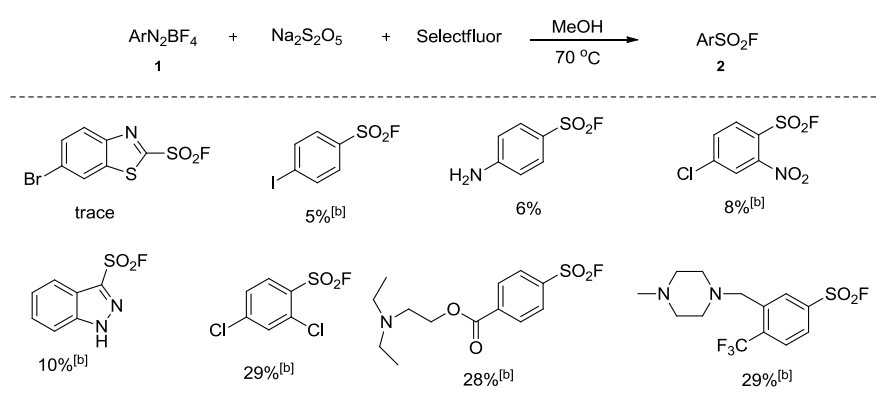

${ }^{a}$ Reaction conditions: 1 ( $0.2 \mathrm{mmol}, 1.0$ eq.), $\mathrm{Na}_{2} \mathrm{~S}_{2} \mathrm{O}_{5}(0.6 \mathrm{mmol}, 3.0$ eq.), Selectfluor $(0.4 \mathrm{mmol}, 2.0$ eq. $)$,

$\mathrm{MeOH}(1.0 \mathrm{~mL}), 70^{\circ} \mathrm{C}$, under $\mathrm{N}_{2}, 9 \mathrm{~h}$, isolated yield. ${ }^{b} \mathrm{Na}_{2} \mathrm{~S}_{2} \mathrm{O}_{5}(0.3 \mathrm{mmol}, 1.5 \mathrm{eq}$.), $\mathrm{Ar}-\mathrm{H}$ was isolated as the major product. 


\section{General Procedure for the Synthesis of sulfonyl fluorides 2}

$$
\underset{1}{\mathrm{ArN}_{2} \mathrm{BF}_{4}}+\mathrm{Na}_{2} \mathrm{~S}_{2} \mathrm{O}_{5}+\text { Selectfluor } \underset{2}{\stackrel{\mathrm{MeOH}, 70}{\circ} \mathrm{C}} \underset{2}{\mathrm{ArSO}_{2} \mathrm{~F}}
$$

General Procedure A: Under $\mathrm{N}_{2}$ atmosphere,a $25 \mathrm{~mL}$ schlenk tube was charged with aryldiazonium salts 1 (0.2 mmol, 1.0 eq), $\mathrm{Na}_{2} \mathrm{~S}_{2} \mathrm{O}_{5}$ (114 mg, $0.6 \mathrm{mmol}, 3.0$ equiv), Selectfluor (142 mg, $0.4 \mathrm{mmol}, 2.0$ equiv) and $\mathrm{MeOH}(1.0 \mathrm{~mL})$. After stirring at $70{ }^{\circ} \mathrm{C}$ (heating block, IKA RCT basic) for $9 \mathrm{~h}$, the reaction mixture was filtered through a pad of celite, eluted with ethyl acetate, concentrated, and purified by flash column chromatography (eluent: petroleum ether/EtOAc) on silica gel to give the desired product 2.

General Procedure B: Under $\mathrm{N}_{2}$ atmosphere,a $25 \mathrm{~mL}$ schlenk tube was charged with aryldiazonium salts 1 (0.2 mmol, 1.0 eq), $\mathrm{Na}_{2} \mathrm{~S}_{2} \mathrm{O}_{5}$ ( $57 \mathrm{mg}, 0.3 \mathrm{mmol}, 1.5$ equiv), Selectfluor (142 mg, $0.4 \mathrm{mmol}, 2.0$ equiv) and $\mathrm{MeOH}\left(1.0 \mathrm{~mL}\right.$ ). After stirring at $70{ }^{\circ} \mathrm{C}$ (heating block, IKA RCT basic) for $9 \mathrm{~h}$, the reaction mixture was filtered through a pad of celite, eluted with ethyl acetate, concentrated, and purified by flash column chromatography (eluent: petroleum ether/EtOAc) on silica gel to give the desired product 2.

\section{General procedure for the one-pot synthesis of sulfonyl fluorides from amines}

To a $25 \mathrm{~mL}$ round-bottom flask equipped with a magnetic stirring bar, t-BuONO (135 uL, $1.0 \mathrm{mmol}, 2.0$ equiv) was added dropwise at $0{ }^{\circ} \mathrm{C}$ to a solution of $\mathrm{ArNH}_{2}\left(0.5 \mathrm{mmol}, 1.0\right.$ equiv) and $\mathrm{HBF}_{4}(132 \mu \mathrm{L}$, aq. $48 \%, 1.0 \mathrm{mmol}, 2.0$ equiv) in $0.75 \mathrm{~mL} \mathrm{EtOH}$. After the mixture was stirred at room temperature for $45 \mathrm{~min}$, the solvents were removed under vacuum at $35^{\circ} \mathrm{C}$. Then $\mathrm{Na}_{2} \mathrm{~S}_{2} \mathrm{O}_{5}(\mathrm{Condition}$ : $0.6 \mathrm{mmol}, 3.0$ equiv or Condition b: $0.3 \mathrm{mmol}, 1.5$ equiv), Selectfluor (142 mg, $0.4 \mathrm{mmol}, 2.0$ equiv) and $\mathrm{MeOH}(1.0 \mathrm{~mL})$ were added successively. After the reaction mixture was refluxed at $70^{\circ} \mathrm{C}$ (heating block, IKA RCT basic) for another $9 \mathrm{~h}$, the mixture was diluted with water and extracted with DCM. The combined organic layers were dried over $\mathrm{Na}_{2} \mathrm{SO}_{4}$, and concentrated under reduced pressure. The residure was purified by flash column chromatography (eluent: petroleum ether/EtOAc) on silica gel to give the desired product 2.

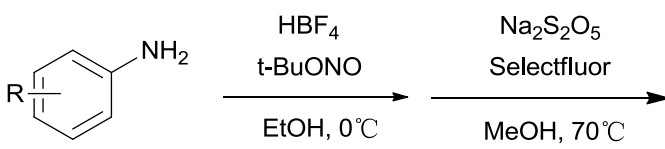

$\mathbf{s}$

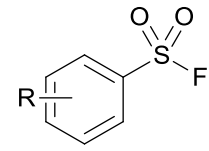

$$
\begin{aligned}
& \mathbf{2 a}^{\mathbf{a}}: \mathrm{R}=4-\mathrm{CH}_{3}, 60 \% \text { yield } \\
& \mathbf{2 b}^{\mathbf{a}}: \mathrm{R}=4-\mathrm{OCH}_{3}, 78 \% \text { yield } \\
& \mathbf{2 h}^{\mathbf{b}}: \mathrm{R}=4-\mathrm{Cl}, 61 \% \text { yield } \\
& \mathbf{2 j}^{\mathbf{b}}: \mathrm{R}=4-\mathrm{NO}_{2}, 56 \% \text { yield }
\end{aligned}
$$




\section{Mechanistic Experiments}

(a) Radical clock experiments<smiles>C=CCOc1ccccc1NC(F)(Br)Br</smiles>

1aj

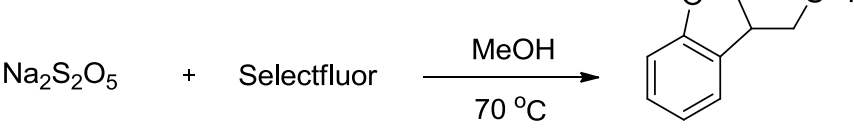

3aj, $40 \%$<smiles>C=CCOc1ccccc1S(=O)(=O)F</smiles>

2aj

not detected

To a $25 \mathrm{~mL}$ schlenk tube equipped with a magnetic stirring bar, aryldiazonium salts 1 aj $\left(49 \mathrm{mg}, 0.2 \mathrm{mmol}, 1.0\right.$ equiv), $\mathrm{Na}_{2} \mathrm{~S}_{2} \mathrm{O}_{5}(114$ $\mathrm{mg}, 0.6 \mathrm{mmol}, 3.0$ equiv), Selectfluor (142 mg, $0.4 \mathrm{mmol}, 2.0$ equiv) and $\mathrm{MeOH}\left(1.0 \mathrm{~mL}\right.$ ) were added successively under $\mathrm{N}_{2}$ atmosphere. The reaction mixture was stirred at $70{ }^{\circ} \mathrm{C}$ (heating block, IKA RCT basic) overnight. After stirring at $70{ }^{\circ} \mathrm{C}$ for $9 \mathrm{~h}$, the reaction mixture was filtered through a pad of celite, eluted with ethyl acetate, concentrated, and purified by flash column chromatography (eluent: petroleum ether/EtOAc) on silica gel to give the product 3 aj in $40 \%$ yield.

\section{(b) Radical trapping experiments ${ }^{5}$}

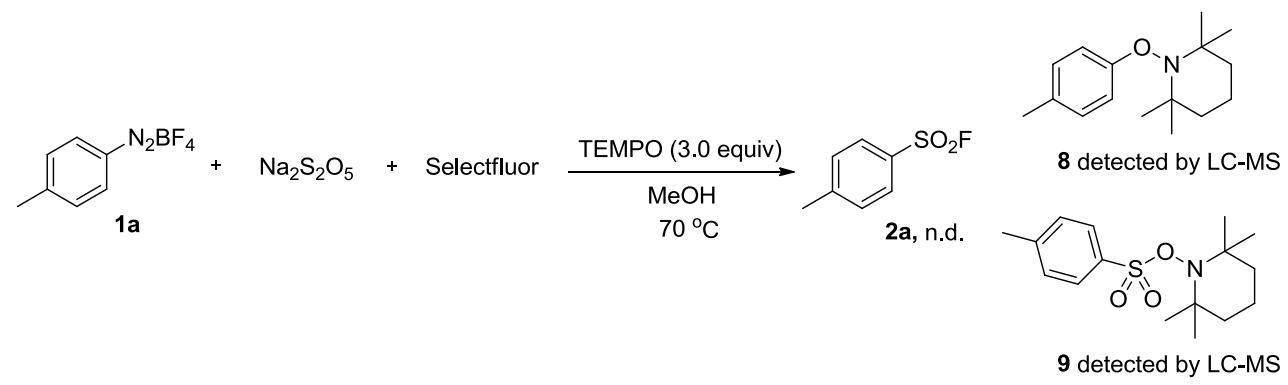

To a $25 \mathrm{~mL}$ schlenk tube equipped with a magnetic stirring bar, aryldiazonium salts $1 \mathrm{a}\left(41 \mathrm{mg}, 0.2 \mathrm{mmol}, 1.0\right.$ equiv), $\mathrm{Na}_{2} \mathrm{~S}_{2} \mathrm{O}_{5}(114$ mg, $0.6 \mathrm{mmol}, 3.0$ equiv), Selectfluor (142 mg, $0.4 \mathrm{mmol}, 2.0$ equiv), TEMPO (94 mg, $0.6 \mathrm{mmol}, 3$ equiv) and MeOH (1.0 mL ) were added successively under $\mathrm{N}_{2}$ atmosphere. The reaction mixture was stirred at $70{ }^{\circ} \mathrm{C}$ (heating block, IKA RCT basic) overnight. TLC and LC-MS analysis demonstrated the sulfonyl fluoride $\mathbf{2 a}$ is not founded, and the aryl radical and arylsulfonyl radical combined with TEMPO were detected by LC-MS.
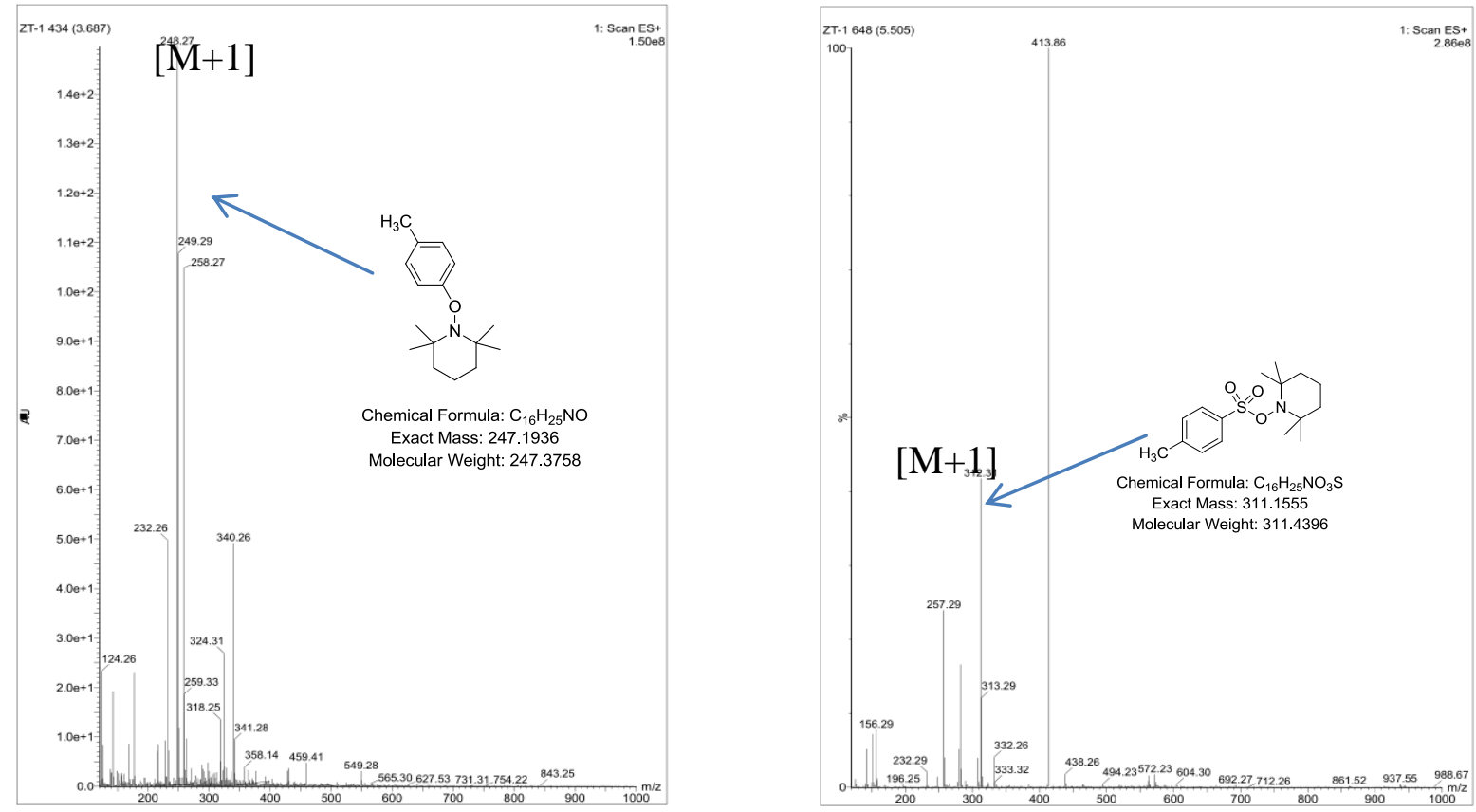


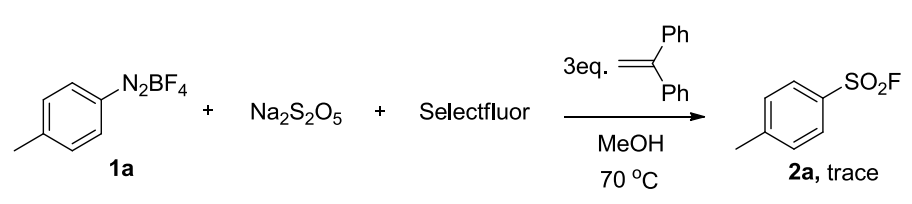<smiles>Cc1ccc(C=C(c2ccccc2)c2ccccc2)cc1</smiles>

6, detected by LC-MS<smiles>Cc1ccc(S(=O)(=O)C=C(c2ccccc2)c2ccccc2)cc1</smiles>

7, detected by LC-MS isolated yield $15 \%$

To a $25 \mathrm{~mL}$ schlenk tube equipped with a magnetic stirring bar, aryldiazonium salts 1 a $\left(41 \mathrm{mg}, 0.2 \mathrm{mmol}, 1.0\right.$ equiv), $\mathrm{Na}_{2} \mathrm{~S}_{2} \mathrm{O}_{5}(114$ mg, $0.6 \mathrm{mmol}, 3.0$ equiv), Selectfluor (142 mg, $0.4 \mathrm{mmol}, 2.0$ equiv), 1,1-diphenylethylene (108 mg, $0.6 \mathrm{mmol}, 3 \mathrm{equiv}$ ) and MeOH $(1.0 \mathrm{~mL})$ were added successively under $\mathrm{N}_{2}$ atmosphere. The reaction mixture was stirred at $70{ }^{\circ} \mathrm{C}$ (heating block, IKA RCT basic) overnight. TLC analysis demonstrated only a small amount of sulfonyl fluoride $2 \mathrm{a}$ was formed, and the (2-(p-tolyl)ethene-1,1-diyl) dibenzene 6 and (2-tosylethene-1,1-diyl)dibenzene 7 were determined by LC-MS. 7 was isolated in 15\% yield.
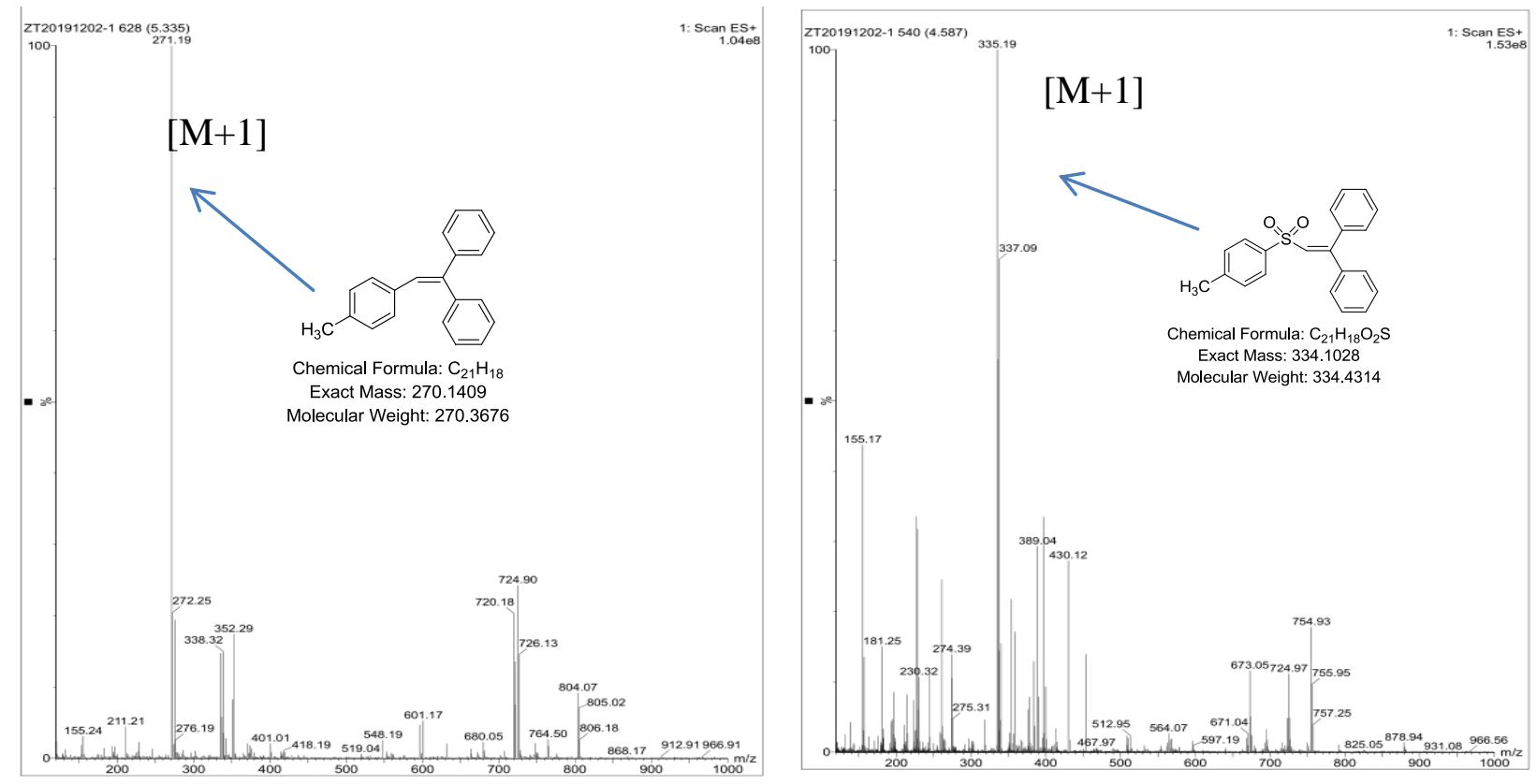

\section{Gram-scale experiment}<smiles>Cc1cc(C)nc(NS(=O)(=O)c2ccc(N([13CH3])C(F)(F)F)cc2)n1</smiles>

1 af, $7 \mathrm{mmol}, 2.64 \mathrm{~g}$

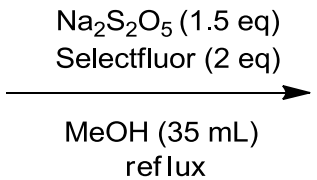

reflux

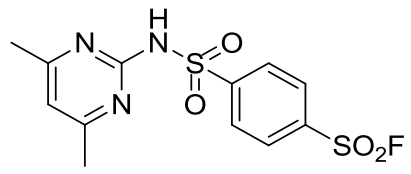

2af, $1.50 \mathrm{~g}, 62 \%$

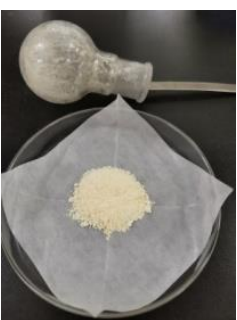

2af

To a $100 \mathrm{~mL}$ round-bottom flask equipped with a magnetic stirring bar, aryldiazonium salts 1 af $\left(2.64 \mathrm{~g}, 7.0 \mathrm{mmol}, 1.0\right.$ equiv), $\mathrm{Na}_{2} \mathrm{~S}_{2} \mathrm{O}_{5}$ (1.996 g, $10.5 \mathrm{mmol}, 1.5$ equiv), Selectfluor (4.96 g, $14 \mathrm{mmol}, 2.0$ equiv) and $\mathrm{MeOH}\left(1.0 \mathrm{~mL}\right.$ ) were added successively under $\mathrm{N}_{2}$ atmosphere. After the reaction mixture was stirred at $70{ }^{\circ} \mathrm{C}$ (oil bath) overnight, the reaction mixture was cooled to room temperature and then diluted with water and extracted with DCM. The combined organic layers were dried over $\mathrm{Na}_{2} \mathrm{SO}_{4}$, and concentrated under reduced pressure. The residue was purified by flash column chromatography (petroleum ether to ethyl acetate 2:1, v/v) on silica gel to afford the pure product 2 af in $62 \%$ yield. 


\section{Characterization of Compounds 2a-2z, 2aa-2ai, 2ak 3aj and s1-s4}<smiles>Cc1ccc(S(=O)(=O)F)cc1</smiles>

\section{4-methylbenzene-1-sulfonyl fluoride (2a)}

Compound 2a was prepared according to General Procedure A. The residue was purified by flash column chromatography on silica (petroleum ether/dichloromethane 30:1) to give a colourless oil (23 mg, 66\% yield). The spectroscopic data were consistent with those previously published. ${ }^{6}$

${ }^{1} \mathrm{H}$ NMR $\left(400 \mathrm{MHz}, \mathrm{CDCl}_{3}\right) \delta 7.88$ (d, $\left.J=7.9 \mathrm{~Hz}, 2 \mathrm{H}\right), 7.42$ (d, $\left.J=7.8 \mathrm{~Hz}, 2 \mathrm{H}\right), 2.49(\mathrm{~s}, 3 \mathrm{H}) ;{ }^{13} \mathrm{C} \mathrm{NMR}\left(101 \mathrm{MHz} \mathrm{CDCl}_{3}\right) \delta 147.5$, 130.7, 130.3, 128.8, 22.2; ${ }^{19} \mathrm{~F}$ NMR (376 $\left.\mathrm{MHz} \mathrm{CDCl}_{3}\right) \delta 66.26$.<smiles>COc1ccc(S(=O)(=O)F)cc1</smiles>

\section{4-methoxybenzene-1-sulfonyl fluoride $(2 b)$}

Compound $\mathbf{2 b}$ was prepared according to General Procedure $\mathbf{A}$. The residue was purified by flash column chromatography on silica (petroleum ether/ethyl acetate 30:1) to give a white solid (30 mg, 79\% yield). The spectroscopic data were consistent with those previously published. ${ }^{6}$

${ }^{1} \mathrm{H}$ NMR $\left(400 \mathrm{MHz}, \mathrm{CDCl}_{3}\right) \delta 7.94(\mathrm{~d}, J=9.0 \mathrm{~Hz}, 2 \mathrm{H}), 7.06(\mathrm{~d}, J=8.9 \mathrm{~Hz}, 2 \mathrm{H}), 3.92(\mathrm{~s}, 3 \mathrm{H}) ;{ }^{13} \mathrm{C} \mathrm{NMR}\left(101 \mathrm{MHz}, \mathrm{CDCl}_{3}\right) \delta 165.6$, $131.4,124.6(\mathrm{~d}, J=24.7 \mathrm{~Hz}), 115.3,56.5 ;{ }^{19} \mathrm{~F} \mathrm{NMR}\left(376 \mathrm{MHz}, \mathrm{CDCl}_{3}\right) \delta 67.28$.<smiles>O=S(=O)(F)c1ccc(Br)cc1</smiles>

\section{4-(tert-butyl)benzene-1-sulfonyl fluoride (2c)}

Compound 2c was prepared according to the General Procedure A. The residue was purified by flash column chromatography on silica (petroleum ether/dichloromethane 30:1) to give a white solid (30.5 mg, $70 \%$ yield). The spectroscopic data were consistent with those previously published. ${ }^{7}$

${ }^{1} \mathrm{H}$ NMR $\left(400 \mathrm{MHz}, \mathrm{CDCl}_{3}\right) \delta 7.93(\mathrm{~d}, J=8.6 \mathrm{~Hz}, 2 \mathrm{H}), 7.63(\mathrm{~d}, J=8.3 \mathrm{~Hz}, 2 \mathrm{H}), 1.37(\mathrm{~s}, 9 \mathrm{H}) ;{ }^{13} \mathrm{C} \mathrm{NMR}\left(101 \mathrm{MHz}^{\mathrm{N}} \mathrm{CDCl}\right)_{3} \delta 160.0$, $130.0(\mathrm{~d}, J=24.1 \mathrm{~Hz}), 128.4,126.8,35.6,31.0 ;{ }^{19} \mathrm{~F} \mathrm{NMR}\left(376 \mathrm{MHz}, \mathrm{CDCl}_{3}\right) \delta 66.19$.<smiles>O=S(=O)(F)c1ccc(-c2ccccc2)cc1</smiles>

\section{[1,1'-biphenyl]-4-sulfonyl fluoride (2d)}

Compound 2d was prepared according to General Procedure A. The residue was purified by flash column chromatography on silica (petroleum ether/ethyl acetate 20:1) to give a white solid (40 mg, 85\% yield). The spectroscopic data were consistent with those previously published. ${ }^{6}$

${ }^{1} \mathrm{H}$ NMR $\left(400 \mathrm{MHz}, \mathrm{CDCl}_{3}\right) \delta 8.08(\mathrm{~d}, J=7.9 \mathrm{~Hz}, 2 \mathrm{H}), 7.82(\mathrm{~d}, J=8.1 \mathrm{~Hz}, 2 \mathrm{H}), 7.63(\mathrm{~d}, J=7.7 \mathrm{~Hz}, 2 \mathrm{H}), 7.50(\mathrm{dt}, J=19.1,7.1 \mathrm{~Hz}$, $3 \mathrm{H}) ;{ }^{13} \mathrm{C}$ NMR $\left(126 \mathrm{MHz}, \mathrm{CDCl}_{3}\right) \delta 148.7,138.6,131.4(\mathrm{~d}, J=24.6 \mathrm{~Hz}), 129.3,129.3,129.0,128.3,127.5 ;{ }^{19} \mathrm{~F} \mathrm{NMR}(376 \mathrm{MHz}$ $\left.\mathrm{CDCl}_{3}\right) \delta 66.52$.<smiles>O=S(=O)(F)c1ccc(Oc2ccccc2)cc1</smiles>

\section{4-phenoxybenzene-1-sulfonyl fluoride (2e)}

Compound $\mathbf{2 e}$ was prepared according to General Procedure A. The residue was purified by flash column chromatography on silica (petroleum ether/ethyl acetate $20: 1$ ) to give a colorless oil (42.4 mg, $84 \%$ yield). The spectroscopic data were consistent with those previously published. ${ }^{8}$

${ }^{1} \mathrm{H}$ NMR $\left(400 \mathrm{MHz}, \mathrm{CDCl}_{3}\right) \delta 8.08(\mathrm{~d}, J=8.0 \mathrm{~Hz}, 2 \mathrm{H}), 7.82(\mathrm{~d}, J=8.2 \mathrm{~Hz}, 2 \mathrm{H}), 7.63(\mathrm{~d}, J=7.6 \mathrm{~Hz}, 2 \mathrm{H}), 7.50(\mathrm{dt}, J=18.8,7.0 \mathrm{~Hz}$, $3 \mathrm{H}) ;{ }^{13} \mathrm{C}$ NMR $\left(101 \mathrm{MHz}, \mathrm{CDCl}_{3}\right) \delta 164.3,154.3,131.0,130.5,125.8(\mathrm{~d}, \mathrm{~J}=24.9 \mathrm{~Hz}), 125.8,120.8,117.7 ;{ }^{19} \mathrm{~F} \mathrm{NMR}(376 \mathrm{MHz}$, $\left.\mathrm{CDCl}_{3}\right) \delta$ 66.52. HRMS-El $(\mathrm{m} / \mathrm{z}):[\mathrm{M}]^{+}$calculated for $\mathrm{C}_{12} \mathrm{H}_{9} \mathrm{O}_{3} \mathrm{FS}^{+}: 252.0251$; found: 252.0249 . 
$\mathrm{SO}_{2} \mathrm{~F}$

\section{4-(benzyloxy)benzene-1-sulfonyl fluoride (2f)}

Compound $\mathbf{2 f}$ was prepared according to General Procedure A. The residue was purified by flash column chromatography on silica (petroleum ether/ethyl acetate 20:1) to give a white solid (39 mg, 73\% yield). The spectroscopic data were consistent with those previously published. ${ }^{8}$

${ }^{1} \mathrm{H}$ NMR $\left(400 \mathrm{MHz}, \mathrm{CDCl}_{3}\right) \delta 7.94(\mathrm{~d}, J=9.0 \mathrm{~Hz}, 2 \mathrm{H}), 7.54-7.30(\mathrm{~m}, 5 \mathrm{H}), 7.14(\mathrm{~d}, J=8.9 \mathrm{~Hz}, 2 \mathrm{H}), 5.18(\mathrm{~s}, 2 \mathrm{H}) ;{ }^{13} \mathrm{C} \mathrm{NMR}(101 \mathrm{MHz}$ $\left.\mathrm{CDCl}_{3}\right) \delta 164.3,135.3,130.9,128.8,128.6,127.5,124.3(\mathrm{~d}, J=24.7 \mathrm{~Hz}), 115.7,70.6 ;{ }^{19} \mathrm{~F} \mathrm{NMR}\left(376 \mathrm{MHz}, \mathrm{CDCl}_{3}\right) \delta 67.30 . \mathrm{HRMS}-\mathrm{El}$ $(\mathrm{m} / \mathrm{z}):[\mathrm{M}]^{+}$calculated for $\mathrm{C}_{13} \mathrm{H}_{11} \mathrm{O}_{3} \mathrm{FS}^{+}: 266.0407$; found: 266.0408 .<smiles>CC(=O)Nc1ccc(S(=O)(=O)F)cc1</smiles>

\section{4-acetamidobenzene-1-sulfonyl fluoride $(2 \mathrm{~g})$}

Compound $\mathbf{2 g}$ was prepared according to General Procedure A. The residue was purified by flash column chromatography on silica (petroleum ether/ethyl acetate 3:1) to give a white solid (33.5 mg, 77\% yield). The spectroscopic data were consistent with those previously published.

${ }^{1} \mathrm{H}$ NMR $\left(400 \mathrm{MHz}, \mathrm{DMSO}-\mathrm{d}_{6}\right) \delta 10.62(\mathrm{~s}, 1 \mathrm{H}), 8.05(\mathrm{~d}, J=8.9 \mathrm{~Hz}, 2 \mathrm{H}), 7.93(\mathrm{~d}, J=8.9 \mathrm{~Hz}, 2 \mathrm{H}), 2.12(\mathrm{~s}, 3 \mathrm{H}) ;{ }^{13} \mathrm{C} \mathrm{NMR}(101 \mathrm{MHz}$, DMSO-d $\left.\mathrm{d}_{6}\right) \delta 169.6,146.4,130.0,124.0(\mathrm{~d}, J=23.5 \mathrm{~Hz}), 119.1,24.2 ;{ }^{19} \mathrm{~F}$ NMR $\left(376 \mathrm{MHz}, \mathrm{DMSO}-\mathrm{d}_{6}\right) \delta 67.54$.<smiles>O=S(=O)(F)c1ccc(Cl)cc1</smiles>

\section{4-chlorobenzene-1-sulfonyl fluoride $(2 \mathrm{~h})$}

Compound $\mathbf{2 h}$ was prepared according to General Procedure $\mathbf{B}$. The residue was purified by flash column chromatography on silica (petroleum ether/dichloromethane 20:1) to give a white solid (23.8 $\mathrm{mg}, 61 \%$ yield). The spectroscopic data were consistent with those previously published. ${ }^{6}$

${ }^{1} \mathrm{H}$ NMR $\left(400 \mathrm{MHz}, \mathrm{CDCl}_{3}\right) \delta 7.95(\mathrm{~d}, J=7.0 \mathrm{~Hz}, 2 \mathrm{H}), 7.61(\mathrm{~d}, J=7.0 \mathrm{~Hz}, 2 \mathrm{H}) ;{ }^{13} \mathrm{C} \mathrm{NMR}\left(101 \mathrm{MHz}, \mathrm{CDCl}_{3}\right) \delta 142.6,131.4(\mathrm{~d}, J=$ $25.8 \mathrm{~Hz}), 130.1,129.8 ;{ }^{19} \mathrm{~F} \mathrm{NMR}\left(376 \mathrm{MHz}, \mathrm{CDCl}_{3}\right) \delta 66.47$.<smiles>O=S(=O)(F)c1ccc(Br)cc1</smiles>

\section{4-bromobenzene-1-sulfonyl fluoride (2i)}

Compound $\mathbf{2} \mathbf{i}$ was prepared according to General Procedure B. The residue was purified by flash column chromatography on silica (petroleum ether/dichloromethane 20:1) to give a white solid $(30.1 \mathrm{mg}, 63 \%$ yield). The spectroscopic data were consistent with those previously published. ${ }^{7}$

${ }^{1} \mathrm{H}$ NMR $\left(500 \mathrm{MHz}, \mathrm{CDCl}_{3}\right) \delta 7.87(\mathrm{~d}, J=8.4 \mathrm{~Hz}, 2 \mathrm{H}), 7.78(\mathrm{~d}, J=8.1 \mathrm{~Hz}, 2 \mathrm{H}) ;{ }^{13} \mathrm{C} \mathrm{NMR}\left(101 \mathrm{MHz}, \mathrm{CDCl}_{3}\right) \delta 133.2,132.0(\mathrm{~d}, J=$ $25.7 \mathrm{~Hz}), 131.4,129.9 ;{ }^{19} \mathrm{~F} \mathrm{NMR}\left(376 \mathrm{MHz}, \mathrm{CDCl}_{3}\right) \delta 66.39$.<smiles>O=[N+]([O-])c1ccc(S(=O)(=O)F)cc1</smiles>

\section{4-nitrobenzene-1-sulfonyl fluoride (2j)}

Compound 2j was prepared according to General Procedure B. The residue was purified by flash column chromatography on silica (petroleum ether/ethyl acetate 40:1) to give a white solid $(24.5 \mathrm{mg}, 60 \%$ yield). The spectroscopic data were consistent with those previously published. ${ }^{9}$

${ }^{1} \mathrm{H}$ NMR $\left(400 \mathrm{MHz}, \mathrm{CDCl}_{3}\right) \delta 8.49(\mathrm{~d}, J=8.1 \mathrm{~Hz}, 2 \mathrm{H}), 8.24(\mathrm{~d}, J=8.7 \mathrm{~Hz}, 2 \mathrm{H}) ;{ }^{13} \mathrm{C} \mathrm{NMR}\left(101 \mathrm{MHz}, \mathrm{CDCl}_{3}\right) \delta 151.9,138.4(\mathrm{~d}, J=$ $27.0 \mathrm{~Hz}), 130.1,125.0 ;{ }^{19} \mathrm{~F} \mathrm{NMR}\left(376 \mathrm{MHz}, \mathrm{CDCl}_{3}\right) \delta 66.19$. 
<smiles>CCOC(=O)c1ccc(S(=O)(=O)F)cc1</smiles>

ethyl 4-(fluorosulfonyl)benzoate (2k)

Compound 2k was prepared according to General Procedure B. The residue was purified by flash column chromatography on silica (petroleum ether/dichloromethane 10:1) to give a white solid (32.5 mg, 70\% yield). The spectroscopic data were consistent with those previously published. ${ }^{6}$

${ }^{1} \mathrm{H}$ NMR $\left(400 \mathrm{MHz}, \mathrm{CDCl}_{3}\right) \delta 8.28(\mathrm{~d}, J=8.7 \mathrm{~Hz}, 2 \mathrm{H}), 8.09(\mathrm{~d}, J=8.5 \mathrm{~Hz}, 2 \mathrm{H}), 4.44(\mathrm{q}, J=7.1 \mathrm{~Hz}, 2 \mathrm{H}), 1.43(\mathrm{t}, J=7.1 \mathrm{~Hz}, 3 \mathrm{H}) ;{ }^{13} \mathrm{C}$ NMR $\left(101 \mathrm{MHz}, \mathrm{CDCl}_{3}\right) \delta 164.8,137.3,137.0(\mathrm{~d}, J=25.4 \mathrm{~Hz}), 131.1,128.9,62.6,14.6 ;{ }^{19} \mathrm{~F} \mathrm{NMR}\left(376 \mathrm{MHz}, \mathrm{CDCl}_{3}\right) \delta 65.80$.<smiles>O=S(=O)(F)c1ccc(C(F)(F)F)cc1</smiles>

\section{4-(trifluoromethyl)benzene-1-sulfonyl fluoride (2I)}

Compound 2I was prepared according to General Procedure B. The residue was purified by flash column chromatography on silica (petroleum ether/dichloromethane 20:1) to give a white solid (23.3 mg, 51\% yield). The spectroscopic data were consistent with those previously published. ${ }^{7}$

${ }^{1} \mathrm{H}$ NMR $\left(400 \mathrm{MHz}, \mathrm{CDCl}_{3}\right) \delta 8.17(\mathrm{~d}, J=8.3 \mathrm{~Hz}, 2 \mathrm{H}), 7.92(\mathrm{~d}, J=8.3 \mathrm{~Hz}, 2 \mathrm{H}) ;{ }^{13} \mathrm{C} \mathrm{NMR}\left(101 \mathrm{MHz}, \mathrm{CDCl}_{3}\right) \delta 137.6-136.1(\mathrm{~m})$, $129.2,127.0(\mathrm{~d}, J=3.5 \mathrm{~Hz}), 124.2,121.5 ;{ }^{19} \mathrm{~F} \mathrm{NMR}\left(376 \mathrm{MHz}, \mathrm{CDCl}_{3}\right) \delta 65.90\left(-\mathrm{SO}_{2} \mathrm{~F}\right),-63.52\left(-\mathrm{CF}_{3}\right)$.<smiles>N#Cc1ccc(S(F)(F)F)cc1</smiles>

\section{4-cyanobenzene-1-sulfonyl fluoride $(2 \mathrm{~m})$}

Compound $\mathbf{2 m}$ was prepared according to General Procedure B. The residue was purified by flash column chromatography on silica (petroleum ether/ethyl acetate 20:1) to give a white solid (23 mg, 62\% yield). The spectroscopic data were consistent with those previously published. ${ }^{10}$

${ }^{1} \mathrm{H}$ NMR $\left(400 \mathrm{MHz}, \mathrm{CDCl}_{3}\right) \delta 8.15(\mathrm{~d}, J=7.4 \mathrm{~Hz}, 2 \mathrm{H}), 7.95(\mathrm{~d}, J=8.1 \mathrm{~Hz}, 2 \mathrm{H}) ;{ }^{13} \mathrm{C} \mathrm{NMR}\left(101 \mathrm{MHz}, \mathrm{CDCl}_{3}\right) \delta 136.9(\mathrm{~d}, J=26.7 \mathrm{~Hz})$, 133.5, 129.2, 119.5, 116.6; ${ }^{19} \mathrm{~F}$ NMR $\left(376 \mathrm{MHz} \mathrm{CDCl}_{3}\right) \delta 65.96$.<smiles>O=C(c1ccccc1)c1ccc(S(=O)(=O)F)cc1</smiles>

\section{4-benzoylbenzene-1-sulfonyl fluoride (2n)}

Compound 2n was prepared according to General Procedure B. The residue was purified by flash column chromatography on silica (petroleum ether/dichloromethane 10:1) to give a white solid (32.8 mg, 62\% yield). The spectroscopic data were consistent with those previously published. ${ }^{8}$

${ }^{1} \mathrm{H}$ NMR $\left(500 \mathrm{MHz}, \mathrm{CDCl}_{3}\right) \delta 8.14(\mathrm{~d}, J=7.1 \mathrm{~Hz}, 2 \mathrm{H}), 7.99(\mathrm{~d}, J=7.0 \mathrm{~Hz}, 2 \mathrm{H}), 7.80(\mathrm{~d}, J=6.7 \mathrm{~Hz}, 2 \mathrm{H}), 7.66(\mathrm{~d}, J=6.5 \mathrm{~Hz}, 1 \mathrm{H}), 7.54$ $(\mathrm{d}, J=6.6 \mathrm{~Hz}, 2 \mathrm{H}) ;{ }^{13} \mathrm{C}$ NMR $\left(101 \mathrm{MHz}, \mathrm{CDCl}_{3}\right) \delta 194.7,144.0,136.0,135.9(\mathrm{~d}, J=25.5 \mathrm{~Hz}), 133.8,130.7,130.2 ;{ }^{19} \mathrm{~F} \mathrm{NMR}(471$ $\left.\mathrm{MHz}, \mathrm{CDCl}_{3}\right) \delta$ 65.95; HRMS-El $(\mathrm{m} / \mathrm{z}):[\mathrm{M}]^{+}$calculated for $\mathrm{C}_{13} \mathrm{H}_{9} \mathrm{O}_{3} \mathrm{FS}^{+}: 264.0251$; found: 264.0250 .<smiles>O=C(O)c1ccc(S(=O)(=O)F)cc1</smiles>

\section{4-(fluorosulfonyl)benzoic acid (20)}

Compound $\mathbf{2 0}$ was prepared according to General Procedure B. The residue was purified by flash column chromatography on silica (petroleum ether/dichloromethane 1:1) to give a white solid (25.3 mg, 62\% yield). The spectroscopic data were consistent with those previously published. ${ }^{11}$

${ }^{1} \mathrm{H}$ NMR $\left(400 \mathrm{MHz}\right.$, DMSO-d $\left.\mathrm{d}_{6}\right) \delta 13.99(\mathrm{~s}, 1 \mathrm{H}), \delta 8.43-7.97(\mathrm{~m}, 4 \mathrm{H}) ;{ }^{13} \mathrm{C}$ NMR $\left(101 \mathrm{MHz}, \mathrm{DMSO}-\mathrm{d}_{6}\right) \delta 165.6,138.0,134.7(\mathrm{~d}, \mathrm{~J}=$ $23.7 \mathrm{~Hz}$ ), $130.9,128.8 ;{ }^{19} \mathrm{~F}$ NMR (376 MHz, DMSO-d 6 ) $\delta 66.03$. 
<smiles>O=S(=O)(F)c1ccccc1Br</smiles>

\section{2-bromobenzene-1-sulfonyl fluoride (2p)}

Compound $2 \mathbf{p}$ was prepared according to General Procedure B. The residue was purified by flash column chromatography on silica (petroleum ether/dichloromethane 10:1) to give a white solid (24.9 mg, 52\% yield). The spectroscopic data were consistent with those previously published. ${ }^{12}$

${ }^{1} \mathrm{H}$ NMR $\left(400 \mathrm{MHz}, \mathrm{CDCl}_{3}\right) \delta 8.14(\mathrm{dd}, J=7.3,2.3 \mathrm{~Hz}, 1 \mathrm{H}), 7.85(\mathrm{dd}, J=7.3,1.8 \mathrm{~Hz}, 1 \mathrm{H}), 7.63-7.52(\mathrm{~m}, 2 \mathrm{H}) ;{ }^{13} \mathrm{C} \mathrm{NMR}(101 \mathrm{MHz}$, $\left.\mathrm{CDCl}_{3}\right) \delta 136.2,136.0,134.0(\mathrm{~d}, J=24.2 \mathrm{~Hz}), 132.2,128.0,121.2 ;{ }^{19} \mathrm{~F} \mathrm{NMR}\left(376 \mathrm{MHz}, \mathrm{CDCl}_{3}\right) \delta 57.94$.<smiles>COc1ccccc1S(=O)(=O)F</smiles>

\section{2-methoxybenzene-1-sulfonyl fluoride (2q)}

Compound $\mathbf{2 q}$ was prepared according to General Procedure A. The residue was purified by flash column chromatography on silica (petroleum ether/dichloromethane 10:1) to give a white solid (20 mg, 53\% yield). The spectroscopic data were consistent with those previously published. ${ }^{10}$

${ }^{1} \mathrm{H}$ NMR $\left(400 \mathrm{MHz}, \mathrm{CDCl}_{3}\right) \delta 7.93(\mathrm{dd}, J=8.1,1.5 \mathrm{~Hz}, 1 \mathrm{H}), 7.76-7.64(\mathrm{~m}, 1 \mathrm{H}), 7.16-7.07(\mathrm{~m}, 2 \mathrm{H}), 4.01(\mathrm{~s}, 3 \mathrm{H}) ;{ }^{13} \mathrm{C} \mathrm{NMR}(101$ $\left.\mathrm{MHz}, \mathrm{CDCl}_{3}\right) \delta 158.5,137.8,131.6,121.8(\mathrm{~d}, \mathrm{~J}=23.2 \mathrm{~Hz}), 120.9,113.1,56.9 ;{ }^{19} \mathrm{~F} \mathrm{NMR}\left(376 \mathrm{MHz}, \mathrm{CDCl}_{3}\right) \delta 58.57$.<smiles>O=S(=O)(F)c1cccc(Br)c1</smiles>

\section{3-bromobenzene-1-sulfonyl fluoride (2r)}

Compound $2 \mathbf{r}$ was prepared according to General Procedure B. The residue was purified by flash column chromatography on silica (petroleum ether/dichloromethane $10: 1)$ to give a colorless oil $(25.4 \mathrm{mg}, 53 \%$ yield). The spectroscopic data were consistent with those previously published. ${ }^{8}$

${ }^{1} \mathrm{H}$ NMR $\left(400 \mathrm{MHz}, \mathrm{CDCl}_{3}\right) \delta 8.15(\mathrm{t}, J=1.7 \mathrm{~Hz}, 1 \mathrm{H}), 7.93(\mathrm{dd}, J=21.0,8.0 \mathrm{~Hz}, 2 \mathrm{H}), 7.52(\mathrm{t}, J=8.0 \mathrm{~Hz}, 1 \mathrm{H}) ;{ }^{13} \mathrm{C} \mathrm{NMR}(101 \mathrm{MHz}$, $\left.\mathrm{CDCl}_{3}\right) \delta 138.8,134.8(\mathrm{~d}, J=25.4 \mathrm{~Hz}), 131.3,131.2,127.0,123.6 ;{ }^{19} \mathrm{~F} \mathrm{NMR}\left(376 \mathrm{MHz}, \mathrm{CDCl}_{3}\right) \delta 66.23$.<smiles>CCOC(=O)c1cccc(S(F)(F)F)c1</smiles>

\section{ethyl 3-(fluorosulfonyl)benzoate (2s)}

Compound $\mathbf{2 s}$ was prepared according to General Procedure B. The residue was purified by flash column chromatography on silica (petroleum ether/ethyl acetate $50: 1$ ) to give a colorless oil ( $30 \mathrm{mg}, 64 \%$ yield).

${ }^{1} \mathrm{H}$ NMR $\left(400 \mathrm{MHz}, \mathrm{CDCl}_{3}\right) \delta 8.66(\mathrm{~s}, 1 \mathrm{H}), 8.44(\mathrm{~d}, J=7.8 \mathrm{~Hz}, 1 \mathrm{H}), 8.18(\mathrm{~d}, J=7.9 \mathrm{~Hz}, 1 \mathrm{H}), 7.74(\mathrm{t}, J=7.9 \mathrm{~Hz}, 1 \mathrm{H}), 4.45(\mathrm{q}, J=7.1$ $\mathrm{Hz}, 2 \mathrm{H}), 1.43(\mathrm{t}, J=7.1 \mathrm{~Hz}, 3 \mathrm{H}) ;{ }^{13} \mathrm{C}$ NMR $\left(101 \mathrm{MHz}, \mathrm{CDCl}_{3}\right) \delta 164.1,136.3,133.6(\mathrm{~d}, J=25.6 \mathrm{~Hz}), 132.4,132.0,130.0,129.5,62.1$, 14.2; ${ }^{19} \mathrm{~F}$ NMR (376 MHz, $\mathrm{CDCl}_{3}$ ) $\delta$ 66.00; HRMS-El $(\mathrm{m} / \mathrm{z})$ : [M] ${ }^{+}$calculated for $\mathrm{C}_{9} \mathrm{H}_{9} \mathrm{O}_{4} \mathrm{FS}^{+}: 232.0200$; found: 232.0201.<smiles>CC(C)Nc1cccc(S(=O)(=O)F)c1</smiles>

\section{3-acetamidobenzene-1-sulfonyl fluoride (2t)}

Compound $2 t$ was prepared according to General Procedure A. The residue was purified by flash column chromatography on silica (petroleum ether/dichloromethane 10:1) to give a white solid $(21.7 \mathrm{mg}, 50 \%$ yield).

${ }^{1} \mathrm{H}$ NMR $\left(400 \mathrm{MHz}, \mathrm{CDCl}_{3}\right) \delta 8.16(\mathrm{~s}, 1 \mathrm{H}), 7.99(\mathrm{~s}, 1 \mathrm{H}), 7.87(\mathrm{~s}, 1 \mathrm{H}), 7.72(\mathrm{~d}, J=7.9 \mathrm{~Hz}, 1 \mathrm{H}), 7.57(\mathrm{t}, J=8.0 \mathrm{~Hz}, 1 \mathrm{H}), 2.24(\mathrm{~s}, 3 \mathrm{H})$; ${ }^{13} \mathrm{C}$ NMR $\left(101 \mathrm{MHz}, \mathrm{CDCl}_{3}\right) \delta 169.1,139.4,133.6(\mathrm{~d}, J=24.5 \mathrm{~Hz}), 130.5,126.4,123.6,119.0,24.6 ;{ }^{19} \mathrm{~F} \mathrm{NMR}\left(376 \mathrm{MHz}, \mathrm{CDCl}_{3}\right) \delta$ 65.61; Melting point: $111-113^{\circ} \mathrm{C}$; HRMS-El $(\mathrm{m} / \mathrm{z}):[\mathrm{M}]^{+}$calculated for $\mathrm{C}_{8} \mathrm{H}_{8} \mathrm{O}_{3} \mathrm{NFS}{ }^{+}: 217.0203$; found: 217.0205 
<smiles>COc1cc(S(=O)(=O)F)cc(OC)c1OC</smiles>

3,4,5-trimethoxybenzene-1-sulfonyl fluoride $(2 \mathrm{u})$

Compound $\mathbf{2} \mathbf{u}$ was prepared according to General Procedure A. The residue was purified by flash column chromatography on silica (petroleum ether/ethyl acetate $20: 1)$ to give a white solid $(42.5 \mathrm{mg}, 85 \%$ yield).

${ }^{1} \mathrm{H}$ NMR $\left(400 \mathrm{MHz}, \mathrm{CDCl}_{3}\right) \delta 7.20(\mathrm{~s}, 2 \mathrm{H}), 3.93(\mathrm{~d}, J=5.2 \mathrm{~Hz}, 9 \mathrm{H}) ;{ }^{13} \mathrm{C} \mathrm{NMR}\left(101 \mathrm{MHz}, \mathrm{CDCl}_{3}\right) \delta 153.7,144.1,127.0(\mathrm{~d}, J=24.6 \mathrm{~Hz})$, 105.9, 61.2, 56.7; ${ }^{19} \mathrm{~F} \mathrm{NMR}\left(376 \mathrm{MHz}, \mathrm{CDCl}_{3}\right) \delta$ 66.60; Melting point: $102-104{ }^{\circ} \mathrm{C}$; HRMS-El $(\mathrm{m} / \mathrm{z})$ : $[\mathrm{M}]^{+}$calculated for $\mathrm{C}_{9} \mathrm{H}_{11} \mathrm{O}_{5} \mathrm{FS}^{+}$: 250.0306; found: 250.0308 .<smiles>Cc1ccc(S(=O)(=O)F)cc1C</smiles>

\section{3,4-dimethylbenzene-1-sulfonyl fluoride (2v)}

Compound 2v was prepared according to General Procedure A. The residue was purified by flash column chromatography on silica (petroleum ether/dichloromethane 20:1) to give a colorless oil (23.4 mg, 62\% yield).

${ }^{1} \mathrm{H}$ NMR $\left(400 \mathrm{MHz}, \mathrm{CDCl}_{3}\right) \delta 7.75(\mathrm{~s}, 1 \mathrm{H}), 7.73(\mathrm{~d}, J=8.1 \mathrm{~Hz}, 1 \mathrm{H}), 7.36(\mathrm{~d}, J=7.9 \mathrm{~Hz}, 1 \mathrm{H}), 2.37(\mathrm{~d}, J=6.7 \mathrm{~Hz}, 6 \mathrm{H}) ;{ }^{13} \mathrm{C} \mathrm{NMR}(126$ $\left.\mathrm{MHz}, \mathrm{CDCl}_{3}\right) \delta 145.9,138.8,130.8,130.2(\mathrm{~d}, J=23.6 \mathrm{~Hz}), 129.1,126.1,20.3,19.9 ;{ }^{19} \mathrm{~F} \mathrm{NMR}\left(376 \mathrm{MHz}, \mathrm{CDCl}_{3}\right) \delta 66.27 . \mathrm{HRMS}-\mathrm{El}$ $(\mathrm{m} / \mathrm{z}):[\mathrm{M}]^{+}$calculated for $\mathrm{C}_{8} \mathrm{H}_{9} \mathrm{O}_{2} \mathrm{FS}^{+}:$188.0302; found: 188.0303 .<smiles>O=S(=O)(F)c1ccc(Cl)c(Cl)c1</smiles>

\section{3,4-dichlorobenzene-1-sulfonyl fluoride (2w)}

Compound $\mathbf{2 w}$ was prepared according to General Procedure $\mathbf{B}$. The residue was purified by flash column chromatography on silica (petroleum ether/dichloromethane 20:1) to give a white solid $(23.4 \mathrm{mg}, 51 \%$ yield). The spectroscopic data were consistent with those previously published. ${ }^{13}$

${ }^{1} \mathrm{H} \mathrm{NMR}\left(400 \mathrm{MHz}, \mathrm{CDCl}_{3}\right) \delta 8.11(\mathrm{~d}, J=2.1 \mathrm{~Hz}, 1 \mathrm{H}), 7.85(\mathrm{dd}, J=8.5,2.0 \mathrm{~Hz}, 1 \mathrm{H}), 7.73(\mathrm{~d}, J=8.5 \mathrm{~Hz}, 1 \mathrm{H}) ;{ }^{13} \mathrm{C} \mathrm{NMR}(101 \mathrm{MHz}$ $\left.\mathrm{CDCl}_{3}\right) \delta 141.6,135.1,132.8(\mathrm{~d}, J=26.7 \mathrm{~Hz}), 132.2,130.7,127.7 ;{ }^{19} \mathrm{~F} \mathrm{NMR}\left(376 \mathrm{MHz}, \mathrm{CDCl}_{3}\right) \delta 66.73$.<smiles>O=S(=O)(F)c1cccc2ccccc12</smiles>

\section{naphthalene-1-sulfonyl fluoride $(2 x)$}

Compound $\mathbf{2 x}$ was prepared according to General Procedure A. The residue was purified by flash column chromatography on silica (petroleum ether/dichloromethane 40:1) to give a white solid (26 mg, 62\% yield). The spectroscopic data were consistent with those previously published. ${ }^{6}$

${ }^{1} \mathrm{H}$ NMR $\left(400 \mathrm{MHz}, \mathrm{CDCl}_{3}\right) \delta 8.62-8.51(\mathrm{~m}, 1 \mathrm{H}), 8.37$ (dd, $\left.J=7.0,2.9 \mathrm{~Hz}, 1 \mathrm{H}\right), 8.23(\mathrm{dd}, J=7.8,2.6 \mathrm{~Hz}, 1 \mathrm{H}), 8.00(\mathrm{dd}, J=7.9,2.6$ $\mathrm{Hz}, 1 \mathrm{H}), 7.78(\mathrm{td}, J=8.6,3.1 \mathrm{~Hz}, 1 \mathrm{H}), 7.69(\mathrm{dt}, J=10.1,5.7 \mathrm{~Hz}, 1 \mathrm{H}), 7.61(\mathrm{td}, J=8.1,2.9 \mathrm{~Hz}, 1 \mathrm{H}) ;{ }^{13} \mathrm{C} \mathrm{NMR}(101 \mathrm{MHz}, \mathrm{CDCl} 3) \delta$ $137.3,134.4,131.5,129.9,129.5,129.3,128.7,128.1,124.6,124.4 ;{ }^{19} \mathrm{~F} \mathrm{NMR}\left(376 \mathrm{MHz}, \mathrm{CDCl}_{3}\right) \delta 62.58$.<smiles>O=S(=O)(F)c1ccc2ccccc2c1</smiles>

naphthalene-2-sulfonyl fluoride (2y)

Compound $2 \mathbf{y}$ was prepared according to General Procedure A. The residue was purified by flash column chromatography on silica (petroleum ether/dichloromethane $30: 1)$ to give a white solid $(21.5 \mathrm{mg}, 51 \%$ yield). The spectroscopic data were consistent with those previously published. ${ }^{10}$

${ }^{1} \mathrm{H}$ NMR $\left(400 \mathrm{MHz}, \mathrm{CDCl}_{3}\right) \delta 8.60(\mathrm{~s}, 1 \mathrm{H}), 8.04(\mathrm{dd}, J=13.7,8.5 \mathrm{~Hz}, 2 \mathrm{H}), 7.99-7.89(\mathrm{~m}, 2 \mathrm{H}), 7.80-7.63(\mathrm{~m}, 2 \mathrm{H}) ;{ }^{13} \mathrm{C} \mathrm{NMR}(126$ $\left.\mathrm{MHz}_{\mathrm{CDCl}}\right) \delta 136.1,131.9,131.0,130.4,130.2,129.9(\mathrm{~d}, J=24.5 \mathrm{~Hz}), 129.7,128.4,128.2,122.2 ;{ }^{19} \mathrm{~F} \mathrm{NMR}\left(376 \mathrm{MHz}, \mathrm{CDCl}_{3}\right) \delta$ 66.33. 
<smiles>COc1ccc(S(F)(F)F)cn1</smiles>

\section{6-methoxypyridine-3-sulfonyl fluoride (2z)}

Compound $\mathbf{2 z}$ was prepared according to General Procedure A. The residue was purified by flash column chromatography on silica (petroleum ether/dichloromethane 10:1) to give a white solid $(24.1 \mathrm{mg}, 63 \%$ yield). The spectroscopic data were consistent with those previously published. ${ }^{6}$

${ }^{1} \mathrm{H}$ NMR $\left(400 \mathrm{MHz}, \mathrm{CDCl}_{3}\right) \delta 8.81(\mathrm{~s}, 1 \mathrm{H}), 8.07(\mathrm{dd}, J=8.8,2.6 \mathrm{~Hz}, 1 \mathrm{H}), 6.92(\mathrm{~d}, J=8.8 \mathrm{~Hz}, 1 \mathrm{H}), 4.05(\mathrm{~s}, 3 \mathrm{H}) ;{ }^{13} \mathrm{C} \mathrm{NMR}(101 \mathrm{MHz}$, $\left.\mathrm{CDCl}_{3}\right) \delta 168.3,149.5,138.0,122.5(\mathrm{~d}, J=22.7 \mathrm{~Hz}), 112.3,54.9 ;{ }^{19} \mathrm{~F} \mathrm{NMR}\left(376 \mathrm{MHz}, \mathrm{CDCl}_{3}\right) \delta 68.56$.<smiles>COC(=O)c1sccc1S(=O)(=O)[O-]</smiles>

\section{methyl 3-(fluorosulfonyl)thiophene-2-carboxylate (2aa)}

Compound 2aa was prepared according to General Procedure B. The residue was purified by flash column chromatography on silica (petroleum ether/dichloromethane 10:1) to give a white solid (15.7 mg, 35\% yield). The spectroscopic data were consistent with those previously published. ${ }^{13}$

${ }^{1} \mathrm{H}$ NMR $\left(400 \mathrm{MHz}, \mathrm{CDCl}_{3}\right) \delta 7.65(\mathrm{~d}, J=5.3 \mathrm{~Hz}, 1 \mathrm{H}), 7.60(\mathrm{~d}, J=5.3 \mathrm{~Hz}, 1 \mathrm{H}), 3.98(\mathrm{~s}, 3 \mathrm{H}) ;{ }^{13} \mathrm{C} \mathrm{NMR}\left(101 \mathrm{MHz}, \mathrm{CDCl}_{3}\right) \delta 159.0$, $137.2,134.5(\mathrm{~d}, J=29.9 \mathrm{~Hz}), 130.9,130.5(\mathrm{~d}, J=2.4 \mathrm{~Hz}), 53.5 ;{ }^{19} \mathrm{~F} \mathrm{NMR}\left(376 \mathrm{MHz}, \mathrm{CDCl}_{3}\right) \delta 62.83$.<smiles>O=S(=O)(F)c1ccc2ncccc2c1</smiles>

\section{quinoline-6-sulfonyl fluoride (2ab)}

Compound 2ab was prepared according to General Procedure A. The residue was purified by flash column chromatography on silica (petroleum ether/ethyl acetate $20: 1$ ) to give a white solid (22 mg, 52\% yield). The spectroscopic data were consistent with those previously published. ${ }^{8}$

${ }^{1} \mathrm{H}$ NMR $\left(400 \mathrm{MHz}, \mathrm{CDCl}_{3}\right) \delta 9.14(\mathrm{~d}, J=3.6 \mathrm{~Hz}, 1 \mathrm{H}), 8.62(\mathrm{~s}, 1 \mathrm{H}), 8.34(\mathrm{t}, J=8.4 \mathrm{~Hz}, 2 \mathrm{H}), 8.17(\mathrm{~d}, J=7.6 \mathrm{~Hz}, 1 \mathrm{H}), 7.62(\mathrm{dd}, J=8.3$ $4.2 \mathrm{~Hz}, 1 \mathrm{H}) ;{ }^{13} \mathrm{C}$ NMR $\left(126 \mathrm{MHz}, \mathrm{CDCl}_{3}\right) \delta 154.8,150.7,137.8,132.3,131.4,131.0(\mathrm{~d}, \mathrm{~J}=25.4 \mathrm{~Hz}), 127.5,126.3,123.6 ;{ }^{19} \mathrm{~F} \mathrm{NMR}$ $\left(376 \mathrm{MHz}, \mathrm{CDCl}_{3}\right) \delta$ 66.39; HRMS-El (m/z): [M] $]^{+}$calculated for $\mathrm{C}_{9} \mathrm{H}_{6} \mathrm{O}_{2} \mathrm{NFS}^{+}:$211.0098; found: 211.0099 .<smiles>O=S(=O)(F)c1ccc2scnc2c1</smiles>

\section{benzo[d]thiazole-5-sulfonyl fluoride (2ac)}

Compound 2ac was prepared according to General Procedure A. The residue was purified by flash column chromatography on silica (petroleum ether/ethyl acetate $30: 1)$ to give a white solid $(21.7 \mathrm{mg}, 50 \%$ yield).

${ }^{1} \mathrm{H} \mathrm{NMR}\left(400 \mathrm{MHz}, \mathrm{CDCl}_{3}\right) \delta 9.23(\mathrm{~s}, 1 \mathrm{H}), 8.80(\mathrm{~d}, J=1.7 \mathrm{~Hz}, 1 \mathrm{H}), 8.23(\mathrm{~d}, J=8.5 \mathrm{~Hz}, 1 \mathrm{H}), 8.04(\mathrm{dd}, J=8.5,1.7 \mathrm{~Hz}, 1 \mathrm{H}) ;{ }^{13} \mathrm{C} \mathrm{NMR}$ $\left(101 \mathrm{MHz}, \mathrm{CDCl}_{3}\right) \delta 157.5,152.9,141.5,131.2(\mathrm{~d}, J=25.5 \mathrm{~Hz}), 124.7,123.9,123.6 ;{ }^{19} \mathrm{~F} \mathrm{NMR}\left(376 \mathrm{MHz}, \mathrm{CDCl}_{3}\right) \delta 67.39 ; \mathrm{Melting}$ point: $140-142{ }^{\circ} \mathrm{C}$; HRMS-El $(\mathrm{m} / \mathrm{z})$ : $[\mathrm{M}]^{+}$calculated for $\mathrm{C}_{7} \mathrm{H}_{4} \mathrm{O}_{2} \mathrm{NS}_{2}{ }^{+}: 216.9662$; found: 216.9663 .<smiles>Cc1cc(=O)oc2cc(S(=O)(=O)OF)ccc12</smiles>

\section{4-methyl-2-oxo-2H-chromene-7-sulfonyl fluoride (2ad)}

Compound 2ad was prepared according to General Procedure B. The residue was purified by flash column chromatography on silica (petroleum ether/dichloromethane $1: 6)$ to give a white solid $(26.7 \mathrm{mg}, 55 \%$ yield).

${ }^{1} \mathrm{H}$ NMR $\left(400 \mathrm{MHz}, \mathrm{CDCl}_{3}\right) \delta 8.00-7.77(\mathrm{~m}, 3 \mathrm{H}), 6.50(\mathrm{~s}, 1 \mathrm{H}), 2.52(\mathrm{~s}, 3 \mathrm{H}) ;{ }^{13} \mathrm{C} \mathrm{NMR}\left(101 \mathrm{MHz}, \mathrm{CDCl}_{3}\right) \delta 158.6,153.2,150.5,135.1$ $(\mathrm{d}, J=26.5 \mathrm{~Hz}), 126.3,125.6,123.3,118.8,117.4,18.7 ;{ }^{19} \mathrm{~F}$ NMR $\left(376 \mathrm{MHz}, \mathrm{CDCl}_{3}\right) \delta 66.34$; Melting point: $129-131^{\circ} \mathrm{C}$; HRMS-El $(\mathrm{m} / \mathrm{z}):[\mathrm{M}]^{+}$calculated for $\mathrm{C}_{10} \mathrm{H}_{7} \mathrm{O}_{4} \mathrm{FS}^{+}: 242.0044$; found: 242.0045 . 
<smiles>CCOC(=O)c1cc2cc(S(=O)(=O)F)ccc2o1</smiles>

ethyl 5-(fluorosulfonyl)benzofuran-2-carboxylate (2ae)

Compound 2ae was prepared according to General Procedure A. The residue was purified by flash column chromatography on silica (petroleum ether/ethyl acetate $20: 1)$ to give a white solid $(33.8 \mathrm{mg}, 62 \%$ yield).

${ }^{1} \mathrm{H} \mathrm{NMR}\left(400 \mathrm{MHz}, \mathrm{CDCl}_{3}\right) \delta 8.44(\mathrm{~d}, J=1.6 \mathrm{~Hz}, 1 \mathrm{H}), 8.08(\mathrm{dd}, J=8.9,1.8 \mathrm{~Hz}, 1 \mathrm{H}), 7.82(\mathrm{~d}, J=8.9 \mathrm{~Hz}, 1 \mathrm{H}), 7.64(\mathrm{~s}, 1 \mathrm{H}), 4.48(\mathrm{q}, J$ $=7.1 \mathrm{~Hz}, 2 \mathrm{H}), 1.45(\mathrm{t}, J=7.1 \mathrm{~Hz}, 3 \mathrm{H}) ;{ }^{13} \mathrm{C}$ NMR $\left(101 \mathrm{MHz}, \mathrm{CDCl}_{3}\right) \delta 158.6,158.4,148.8,128.9(\mathrm{~d}, J=25.1 \mathrm{~Hz}), 127.8,127.0,125.2$, 114.1, 113.4, 62.4, 14.3; ${ }^{19} \mathrm{~F}$ NMR (376 MHz, $\left.\mathrm{CDCl}_{3}\right) \delta 67.65$; Melting point: $90-92{ }^{\circ} \mathrm{C}$; HRMS-El $(\mathrm{m} / \mathrm{z})$ : [M] ${ }^{+}$calculated for $\mathrm{C}_{11} \mathrm{H}_{9} \mathrm{O}_{5} \mathrm{FS}^{+}: 272.0149$; found: 272.0148 .<smiles>Cc1cc(C)nc(NS(=O)(=O)c2ccc(S(=O)(=O)F)cc2)n1</smiles>

4-(N-(4,6-dimethylpyrimidin-2-yl)sulfamoyl)benzene-1-sulfonyl fluoride (2af)

Compound 2af was prepared according to General Procedure B. The residue was purified by flash column chromatography on silica (petroleum ether/ethyl acetate 2:1) to give a faint yellow solid $(41.4 \mathrm{mg}, 60 \%$ yield). The spectroscopic data were consistent with those previously published. ${ }^{8}$

${ }^{1} \mathrm{H}$ NMR $\left(400 \mathrm{MHz}, \mathrm{DMSO}-\mathrm{d}_{6}\right) \delta 13.02(\mathrm{~s}, 1 \mathrm{H}), 8.27$ (s, 4H), $6.72(\mathrm{~s}, 1 \mathrm{H}), 2.25(\mathrm{~s}, 6 \mathrm{H}) ;{ }^{13} \mathrm{C}$ NMR $\left(126 \mathrm{MHz}, \mathrm{DMSO}-\mathrm{d}_{6}\right) \delta 167.3,155.3$, 149.8, $133.7(\mathrm{~d}, J=24.0 \mathrm{~Hz}), 129.4,128.6,111.2,21.9 ;{ }^{19} \mathrm{~F}$ NMR $\left(376 \mathrm{MHz}\right.$, DMSO-d 6 ) $\delta 66.25$; HRMS-ESI $(\mathrm{m} / \mathrm{z}):[\mathrm{M}+\mathrm{H}]^{+} \mathrm{calculated}$ for $\mathrm{C}_{12} \mathrm{H}_{13} \mathrm{FN}_{3} \mathrm{O}_{4} \mathrm{~S}_{2}^{+}: 346.0326$; found: 346.0320 .<smiles>O=S(=O)(F)c1ccc(OCc2ccccn2)c(Cl)c1</smiles>

\section{3-chloro-4-(pyridin-2-ylmethoxy)benzene-1-sulfonyl fluoride (2ag)}

Compound 2ag was prepared according to General Procedure B. The residue was purified by flash column chromatography on silica (petroleum ether/ethyl acetate $5: 1)$ to give a white solid $(32.2 \mathrm{mg}, 53 \%$ yield).

${ }^{1} \mathrm{H} \mathrm{NMR}\left(400 \mathrm{MHz}, \mathrm{CDCl}_{3}\right) \delta 8.61(\mathrm{~d}, J=4.2 \mathrm{~Hz}, 1 \mathrm{H}), 8.03(\mathrm{~d}, J=2.2 \mathrm{~Hz}, 1 \mathrm{H}), 7.86(\mathrm{dd}, J=8.8,2.1 \mathrm{~Hz}, 1 \mathrm{H}), 7.77(\mathrm{td}, J=7.7,1.3 \mathrm{~Hz}$, $1 \mathrm{H}), 7.57(\mathrm{~d}, J=7.8 \mathrm{~Hz}, 1 \mathrm{H}), 7.34-7.22(\mathrm{~m}, 1 \mathrm{H}), 7.18(\mathrm{~d}, J=8.8 \mathrm{~Hz}, 1 \mathrm{H}), 5.38(\mathrm{~s}, 2 \mathrm{H}) ;{ }^{13} \mathrm{C} \mathrm{NMR}\left(101 \mathrm{MHz}^{\mathrm{C}} \mathrm{CDCl}\right)_{3} \delta 1_{159.6,155.1}$, 149.5, 137.3, 130.6, 129.2, $125.3(\mathrm{~d}, J=25.9 \mathrm{~Hz}), 124.6,123.4,121.4,113.6,71.9 ;{ }^{19} \mathrm{~F} \mathrm{NMR}\left(376 \mathrm{MHz}, \mathrm{CDCl}_{3}\right) \delta 67.45 ; \mathrm{Melting}^{2}$ point: $123-125^{\circ} \mathrm{C}$; HRMS-ESI $(\mathrm{m} / \mathrm{z}):[\mathrm{M}+\mathrm{H}]^{+}$calculated for $\mathrm{C}_{12} \mathrm{H}_{10} \mathrm{CIFNO}_{3} \mathrm{~S}^{+}$: 302.0048 ; found: 302.0044 .<smiles>CC(C)[C@H]1CC[C@@H](C)C[C@H]1OC(=O)c1ccc(S(=O)(=O)O)cc1</smiles>

\section{(1R,2S,5R)-2-isopropyl-5-methylcyclohexyl 4-(fluorosulfonyl)benzoate (2ah)}

Compound 2ah was prepared according to General Procedure B. The residue was purified by flash column chromatography on silica (petroleum ether/dichloromethane 6:1) to give a white solid (44.5 mg, 65\% yield). The spectroscopic data were consistent with those previously published. ${ }^{8}$

${ }^{1} \mathrm{H} \mathrm{NMR}\left(400 \mathrm{MHz}, \mathrm{CDCl}_{3}\right) \delta 8.27(\mathrm{~d}, J=8.4 \mathrm{~Hz}, 2 \mathrm{H}), 8.09(\mathrm{~d}, J=8.4 \mathrm{~Hz}, 2 \mathrm{H}), 4.99(\mathrm{td}, J=10.9,4.4 \mathrm{~Hz}, 1 \mathrm{H}), 2.12(\mathrm{~d}, J=11.7 \mathrm{~Hz}$, $1 \mathrm{H}), 1.96-1.85(\mathrm{~m}, 1 \mathrm{H}), 1.76(\mathrm{dd}, J=9.2,6.9 \mathrm{~Hz}, 2 \mathrm{H}), 1.62-1.53(\mathrm{~m}, 2 \mathrm{H}), 1.19-1.08(\mathrm{~m}, 2 \mathrm{H}), 1.00-0.97(\mathrm{~m}, 1 \mathrm{H}), 0.93(\mathrm{t}, J=7.2$ $\mathrm{Hz}, 6 \mathrm{H}), 0.80(\mathrm{~d}, J=6.9 \mathrm{~Hz}, 3 \mathrm{H}) \cdot{ }^{13} \mathrm{C} N M R\left(101 \mathrm{MHz}, \mathrm{CDCl}_{3}\right) \delta 164.0,137.3,136.5(\mathrm{~d}, J=25.3 \mathrm{~Hz}), 130.8,128.6,76.5,47.3,40.9$, $34.3,31.6,26.7,23.7,22.1,20.8,16.6 ;{ }^{19} \mathrm{~F} \mathrm{NMR}\left(376 \mathrm{MHz} \mathrm{CDCl}_{3}\right) \delta 65.85$. 
<smiles>CC12CCC3c4ccc(OC(=O)c5ccc(S(=O)(=O)O)cc5)cc4CCC3C1CCC2=O</smiles>

(8R,9S,13S,14S)-13-methyl-17-oxo-7,8,9,11,12,13,14,15,16,17-decahydro-6H-cyclopenta[a]phenanthren-3-yl 4-(fluorosulfonyl) benzoate (2ai)

Compound 2ai was prepared according to General Procedure B. The residue was purified by flash column chromatography on silica (petroleum ether/ ethyl acetate 20:1) to give a white solid (37.4 $\mathrm{mg}, 41 \%$ yield).

${ }^{1} \mathrm{H}$ NMR $\left(400 \mathrm{MHz}, \mathrm{CDCl}_{3}\right) \delta 8.43(\mathrm{~d}, J=8.3 \mathrm{~Hz}, 2 \mathrm{H}), 8.16(\mathrm{~d}, J=8.4 \mathrm{~Hz}, 2 \mathrm{H}), 7.37(\mathrm{~d}, J=8.5 \mathrm{~Hz}, 1 \mathrm{H}), 7.10-6.91(\mathrm{~m}, 2 \mathrm{H}), 3.03-$ $2.87(\mathrm{~m}, 2 \mathrm{H}), 2.52(\mathrm{dd}, J=18.8,8.6 \mathrm{~Hz}, 1 \mathrm{H}), 2.43(\mathrm{dd}, J=12.8,3.6 \mathrm{~Hz}, 1 \mathrm{H}), 2.32(\mathrm{dd}, J=15.8,5.9 \mathrm{~Hz}, 1 \mathrm{H}), 2.17(\mathrm{dd}, J=18.5,9.2$ $\mathrm{Hz}, 1 \mathrm{H}), 2.13-1.94(\mathrm{~m}, 3 \mathrm{H}), 1.71-1.58(\mathrm{~m}, 2 \mathrm{H}), 1.58-1.42(\mathrm{~m}, 4 \mathrm{H}), 0.93(\mathrm{~s}, 3 \mathrm{H}) ;{ }^{13} \mathrm{C} \mathrm{NMR}\left(101 \mathrm{MHz}, \mathrm{CDCl}_{3}\right) \delta 220.8,163.4$, 148.4, 138.5, 138.2, 137.2 (d, $J=25.5 \mathrm{~Hz}$ ), 136.2, 131.3, 128.7, 126.8, 121.4, 118.6, 50.5, 48.0, 44.3, 38.0, 35.9, 31.6, 29.5, 26.4, 25.8, 21.7, 13.9; $\left.{ }^{19} \mathrm{~F} \mathrm{NMR} \mathrm{(376} \mathrm{MHz,} \mathrm{CDCl}_{3}\right) \delta 65.88$; Melting point could not be measured due to the decomposition temperature lower than melting point; HRMS-El $(\mathrm{m} / \mathrm{z})$ : $[\mathrm{M}]^{+}$calculated for $\mathrm{C}_{25} \mathrm{H}_{25} \mathrm{O}_{5} \mathrm{FS}^{+}: 456.1401$; found: 456.1403 .<smiles>O=S(=O)(F)CC1COc2ccccc21</smiles>

\section{(2,3-dihydrobenzofuran-3-yl)methanesulfonyl fluoride (3aj)}

Compound 3aj was prepared according to General Procedure A. The residue was purified by flash column chromatography on silica (petroleum ether/ ethyl acetate 30:1) to give a white solid (17.3 mg, 40\% yield). The spectroscopic data were consistent with those previously published. ${ }^{8}$

${ }_{1}^{1} \mathrm{H}$ NMR $\left(400 \mathrm{MHz}, \mathrm{CDCl}_{3}\right) \delta 7.41-6.96(\mathrm{~m}, 2 \mathrm{H}), 6.94(\mathrm{t}, J=7.5 \mathrm{~Hz}, 1 \mathrm{H}), 6.87(\mathrm{~d}, J=8.3 \mathrm{~Hz}, 1 \mathrm{H}), 4.75(\mathrm{t}, J=9.2 \mathrm{~Hz}, 1 \mathrm{H}), 4.55(\mathrm{dd}$, $J=9.8,5.5 \mathrm{~Hz}, 1 \mathrm{H}), 4.10(\mathrm{ddd}, J=13.7,9.1,4.5 \mathrm{~Hz}, 1 \mathrm{H}), 3.76(\mathrm{dt}, J=14.8,2.8 \mathrm{~Hz}, 1 \mathrm{H}), 3.66-3.49(\mathrm{~m}, 1 \mathrm{H}) ;{ }^{13} \mathrm{C} \mathrm{NMR}(101 \mathrm{MHz}$, $\left.\mathrm{CDCl}_{3}\right) \delta 159.7,130.1,125.5,124.3,121.4,110.6,75.1,54.8(\mathrm{~d}, J=14.5 \mathrm{~Hz}), 37.8 ;{ }^{19} \mathrm{~F} \mathrm{NMR}\left(376 \mathrm{MHz}, \mathrm{CDCl}_{3}\right) \delta 56.66 ; \mathrm{HRMS}-\mathrm{El}$ $(\mathrm{m} / \mathrm{z}):[\mathrm{M}]^{+}$calculated for $\mathrm{C}_{9} \mathrm{H}_{9} \mathrm{O}_{3} \mathrm{FS}^{+}: 216.0251$; found: 216.0252 .<smiles>COc1c(Br)cc(-c2nnc(-c3ccc(S(F)(F)F)cc3)o2)cc1Br</smiles>

\section{4-(5-(3,5-dibromo-4-methoxyphenyl)-1,3,4-oxadiazol-2-yl)benzene-1-sulfonyl fluoride (2ak)}

Compound 2ak was prepared from the amine s4 $(94 \mathrm{mg}, 0.22 \mathrm{mmol}$ ) according to the procedure in Section 6 . The residue was purified by flash column chromatography on silica (petroleum ether/ ethyl acetate 20:1) to give the desired product as a white solid (46.8 mg, $43 \%$ yield). The spectroscopic data were consistent with previously published. ${ }^{4 \mathrm{~b}}$

${ }^{1} \mathrm{H}$ NMR $\left(400 \mathrm{MHz}, \mathrm{CDCl}_{3}\right) \delta 8.41(\mathrm{~d}, \mathrm{~J}=8.2 \mathrm{~Hz}, 2 \mathrm{H}), 8.32(\mathrm{~s}, 2 \mathrm{H}), 8.20(\mathrm{~d}, \mathrm{~J}=8.6 \mathrm{~Hz}, 2 \mathrm{H}), 3.98(\mathrm{~s}, 3 \mathrm{H}) .{ }^{13} \mathrm{C} \mathrm{NMR}(101 \mathrm{MHz}, \mathrm{CDCl} 3)$ $\delta 163.2,163.1,157.7,135.9(\mathrm{~d}, J=26.1 \mathrm{~Hz}), 131.4,130.0,129.5,128.1,121.5,119.4,61.1 ;{ }^{19} \mathrm{~F} \mathrm{NMR}\left(376 \mathrm{MHz} \mathrm{CDCl}_{3}\right) \delta 66.14$.<smiles>Cc1ccc(S(=O)(=O)C=C(c2ccccc2)c2ccccc2)cc1</smiles>

\section{(2-tosylethene-1,1-diyl)dibenzene (7)}

Compound 7 was obtainded under the standard conditions and 3.0 equiv. of 1,1-diphenylethylene was added. The residue was purified by flash column chromatography on silica (petroleum ether/ ethyl acetate 30:1) to give a colourless oil. (10 mg, $15 \%$ yield). The spectroscopic data were consistent with those previously published. ${ }^{14}$

${ }^{1} \mathrm{H}$ NMR $\left(400 \mathrm{MHz}, \mathrm{CDCl}_{3}\right) \delta 7.48(\mathrm{~d}, J=8.3 \mathrm{~Hz}, 2 \mathrm{H}), 7.36$ (ddd, $\left.J=4.4,3.5,1.5 \mathrm{~Hz}, 2 \mathrm{H}\right), 7.30(\mathrm{t}, J=7.4 \mathrm{~Hz}, 4 \mathrm{H}), 7.23-7.17(\mathrm{~m}$, $2 \mathrm{H}), 7.15(\mathrm{~d}, J=8.1 \mathrm{~Hz}, 2 \mathrm{H}), 7.12-7.07(\mathrm{~m}, 2 \mathrm{H}), 6.99(\mathrm{~s}, 1 \mathrm{H}), 2.38(\mathrm{~s}, 3 \mathrm{H}) ;{ }^{13} \mathrm{C} \mathrm{NMR}\left(101 \mathrm{MHz}, \mathrm{CDCl}_{3}\right) \delta 154.8,143.8,139.3,138.7$, $135.7,130.3,129.9,129.4,129.0,128.9,128.7,128.3,127.9,127.8,21.7$. 
<smiles>C=CCOc1ccccc1N</smiles>

\section{2-(allyloxy)aniline (s1)}

This compound was prepared according to the procedure in Section 3. The spectroscopic data were consistent with those previously published. ${ }^{2}$

${ }^{1} \mathrm{H}$ NMR $\left(400 \mathrm{MHz}, \mathrm{CDCl}_{3}\right) \delta 6.83(\mathrm{~m}, 2 \mathrm{H}), 6.77-6.68(\mathrm{~m}, 2 \mathrm{H}), 6.10$ (ddd, $\left.J=22.5,10.5,5.3 \mathrm{~Hz}, 1 \mathrm{H}\right), 5.43(\mathrm{dd}, J=17.3,1.5 \mathrm{~Hz}, 1 \mathrm{H})$, $5.30(\mathrm{dd}, J=10.5,1.3 \mathrm{~Hz}, 1 \mathrm{H}), 4.58(\mathrm{~d}, J=5.3 \mathrm{~Hz}, 2 \mathrm{H}), 3.71(\mathrm{~s}, 2 \mathrm{H}) ;{ }^{13} \mathrm{C} \mathrm{NMR}\left(126 \mathrm{MHz}, \mathrm{CDCl}_{3}\right) \delta 146.3,136.5,133.6,121.4,118.4$, $117.5,115.3,112.1,69.3$.<smiles>CC(C)[C@H]1CC[C@@H](C)C[C@H]1OC(=O)c1ccc([N+](=O)[O-])cc1</smiles>

\section{(1R,2S,5R)-2-isopropyl-5-methylcyclohexyl 4-nitrobenzoate (4)}

This compound was prepared according to the procedure in Section 3. The spectroscopic data were consistent with those previously published. ${ }^{3}$

${ }^{1} \mathrm{H}$ NMR $\left(400 \mathrm{MHz}, \mathrm{CDCl}_{3}\right) \delta 8.32-8.24(\mathrm{~m}, 2 \mathrm{H}), 8.24-8.16(\mathrm{~m}, 2 \mathrm{H}), 4.97(\mathrm{td}, J=10.9,4.4 \mathrm{~Hz}, 1 \mathrm{H}), 2.18-2.07(\mathrm{~m}, 1 \mathrm{H}), 1.91(\mathrm{dtd}$, $J=13.9,7.0,2.7 \mathrm{~Hz}, 1 \mathrm{H}), 1.81-1.67(\mathrm{~m}, 2 \mathrm{H}), 1.64-1.48(\mathrm{~m}, 2 \mathrm{H}), 1.21-1.06(\mathrm{~m}, 2 \mathrm{H}), 0.95-0.91(\mathrm{~m}, 7 \mathrm{H}), 0.79(\mathrm{~d}, J=7.0 \mathrm{~Hz}$ $3 \mathrm{H}) ;{ }^{13} \mathrm{C}$ NMR $\left(101 \mathrm{MHz}, \mathrm{CDCl}_{3}\right) \delta 164.3,150.5,136.3,130.7,123.6,76.2,47.3,40.9,34.3,31.5,26.7,23.7,22.1,20.8,16.6$.<smiles>CC(C)[C@H]1CC[C@@H](C)C[C@H]1OC(=O)c1ccc(N)cc1</smiles>

\section{(1R,2S,5R)-2-isopropyl-5-methylcyclohexyl 4-aminobenzoate (s2)}

This compound was prepared according to the procedure in Section 3. The spectroscopic data were consistent with those previously published. $^{3}$

${ }^{1} \mathrm{H}$ NMR $\left(400 \mathrm{MHz}, \mathrm{CDCl}_{3}\right) \delta 7.85(\mathrm{~d}, J=8.4 \mathrm{~Hz}, 2 \mathrm{H}), 6.63(\mathrm{~d}, J=8.4 \mathrm{~Hz}, 2 \mathrm{H}), 4.87(\mathrm{td}, J=10.8,4.2 \mathrm{~Hz}, 1 \mathrm{H}), 3.97(\mathrm{~s}, 2 \mathrm{H}), 2.10(\mathrm{~d}, J$ $=11.7 \mathrm{~Hz}, 1 \mathrm{H}), 2.03-1.89(\mathrm{~m}, 1 \mathrm{H}), 1.70(\mathrm{~d}, J=11.4 \mathrm{~Hz}, 2 \mathrm{H}), 1.51(\mathrm{t}, J=11.1 \mathrm{~Hz}, 2 \mathrm{H}), 1.18-1.02(\mathrm{~m}, 2 \mathrm{H}), 0.98-0.84(\mathrm{~m}, 7 \mathrm{H})$, $0.78(\mathrm{~d}, J=6.9 \mathrm{~Hz}, 3 \mathrm{H}) ;{ }^{13} \mathrm{C}$ NMR $\left(101 \mathrm{MHz} \mathrm{CDCl}_{3}\right) \delta 166.3,150.8,131.5,120.2,113.8,74.0,47.3,41.1,34.4,31.4,26.5,23.7$, $22.1,20.8,16.6$.<smiles>CC12CCC3c4ccc(OC(=O)c5ccc([N+](=O)[O-])cc5)cc4CCC3C1CCC2=O</smiles>

(8R,9S,13S,14S)-13-methyl-17-oxo-7,8,9,11,12,13,14,15,16,17-decahydro-6H-cyclopenta[a]phenanthren-3-yl 4-nitrobenzoate $(5)^{15}$

This compound was prepared according to the procedure in Section 3 .

${ }^{1} \mathrm{H}$ NMR $\left(400 \mathrm{MHz}, \mathrm{CDCl}_{3}\right) \delta 8.40-8.31(\mathrm{~m}, 4 \mathrm{H}), 7.36(\mathrm{~d}, J=8.5 \mathrm{~Hz}, 1 \mathrm{H}), 7.04-6.89(\mathrm{~m}, 2 \mathrm{H}), 3.01-2.89(\mathrm{~m}, 2 \mathrm{H}), 2.62-2.39(\mathrm{~m}$, $2 \mathrm{H}), 2.33(\mathrm{td}, J=10.9,3.6 \mathrm{~Hz}, 1 \mathrm{H}), 2.27-1.89(\mathrm{~m}, 4 \mathrm{H}), 1.77-1.41(\mathrm{~m}, 6 \mathrm{H}), 0.93(\mathrm{~s}, 1 \mathrm{H}) ;{ }^{13} \mathrm{C} \mathrm{NMR}\left(101 \mathrm{MHz}, \mathrm{CDCl}_{3}\right) \delta 220.8,163.7$, 150.9, 148.5, 138.4, 138.1, 135.1, 131.3, 126.7, 123.8, 121.5, 118.6, 50.5, 48.0, 44.2, 38.0, 35.9, 31.6, 29.5, 26.4, 25.8, 21.7, 13.9. HRMS-ESI $(\mathrm{m} / \mathrm{z}):[\mathrm{M}+\mathrm{H}]^{+}$calculated for $\mathrm{C}_{25} \mathrm{H}_{26} \mathrm{NO}_{5}{ }^{+}: 420.1805$; found: 420.1811 . 
<smiles>CC12CCC3c4ccc(OC(=O)c5ccc(N)cc5)cc4CCC3C1CCC2=O</smiles>

(8R,9S,13S,14S)-13-methyl-17-oxo-7,8,9,11,12,13,14,15,16,17-decahydro-6H-cyclopenta[a]phenanthren-3-yl 4-aminobenzoate $(\mathrm{s} 3)^{1 \mathrm{c}}$

This compound was prepared according to the procedure in Section 3.

${ }^{1} \mathrm{H}$ NMR $\left(500 \mathrm{MHz}, \mathrm{DMSO}-\mathrm{d}_{6}\right) \delta 7.76(\mathrm{~d}, J=8.6 \mathrm{~Hz}, 1 \mathrm{H}), 7.31(\mathrm{~d}, J=8.5 \mathrm{~Hz}, 1 \mathrm{H}), 6.92(\mathrm{dd}, J=8.4,2.3 \mathrm{~Hz}, 1 \mathrm{H}), 6.87(\mathrm{~d}, J=2.2 \mathrm{~Hz}$, $1 \mathrm{H}), 6.62(\mathrm{~d}, J=8.7 \mathrm{~Hz}, 1 \mathrm{H}), 6.14(\mathrm{~s}, 1 \mathrm{H}), 2.92-2.76(\mathrm{~m}, 1 \mathrm{H}), 2.51-2.33(\mathrm{~m}, 2 \mathrm{H}), 2.25(\mathrm{dd}, J=13.7,6.9 \mathrm{~Hz}, 1 \mathrm{H}), 2.15-2.01(\mathrm{~m}$, $1 \mathrm{H}), 1.96(\mathrm{dd}, J=13.8,11.7 \mathrm{~Hz}, 1 \mathrm{H}), 1.78(\mathrm{~d}, J=11.4 \mathrm{~Hz}, 1 \mathrm{H}), 1.65-1.26(\mathrm{~m}, 3 \mathrm{H}), 0.85(\mathrm{~s}, 2 \mathrm{H}) ;{ }^{13} \mathrm{C} \mathrm{NMR}\left(101 \mathrm{MHz}, \mathrm{DMSO}-\mathrm{d}_{6}\right) \delta$ 219.6, 164.7, 154.1, 148.8, 137.6, 136.7, 131.8, 126.1, 121.7, 119.1, 114.6, 112.7, 49.5, 47.3, 43.5, 37.5, 35.3, 31.3, 28.8, 25.8, 25.3, 21.1, 13.4. HRMS-ESI $(\mathrm{m} / \mathrm{z}):[\mathrm{M}+\mathrm{H}]^{+}$calculated for $\mathrm{C}_{25} \mathrm{H}_{28} \mathrm{NO}_{3}{ }^{+}: 390.2064$; found: 390.2068 .<smiles>COc1c(Br)cc(-c2nnc(-c3ccc(N)cc3)o2)cc1Br</smiles>

4-(5-(3,5-dibromo-4-methoxyphenyl)-1,3,4-oxadiazol-2-yl)aniline (s4)

This compound was prepared according to the procedure in Section 3 .

${ }^{1} \mathrm{H}$ NMR $\left(400 \mathrm{MHz}\right.$, DMSO-d $\left.\mathrm{d}_{6}\right) \delta 8.31(\mathrm{~s}, 2 \mathrm{H}), 7.82(\mathrm{~d}, J=8.7 \mathrm{~Hz}, 2 \mathrm{H}), 6.70(\mathrm{~d}, J=8.7 \mathrm{~Hz}, 2 \mathrm{H}), 5.99(\mathrm{~s}, 2 \mathrm{H}), 3.89(\mathrm{~s}, 3 \mathrm{H}) .{ }^{13} \mathrm{C} \mathrm{NMR}$ $\left(126 \mathrm{MHz}\right.$, DMSO-d $\left.\mathrm{d}_{6}\right) \delta 165.3,160.1,155.8,152.6,130.4,128.5,122.6,118.5,113.5,109.2,60.7 ; \mathrm{HRMS}-\mathrm{ESI}(\mathrm{m} / \mathrm{z}):[\mathrm{M}+\mathrm{H}]^{+}$ calculated for $\mathrm{C}_{15} \mathrm{H}_{12} \mathrm{Br}_{2} \mathrm{~N}_{3} \mathrm{O}_{2}{ }^{+}$: 423.9291 ; found: 423.9288 . 


\section{References}

1. (a) C. Lian, G. Yue, J. Mao, D. Liu, Y. Ding, Z. Liu, D. Qiu, X. Zhao, K. Lu, M. Fagnoni and S. Protti, Org. Lett., 2019, 21, 5187; (b) J. Wu, Y. Gu, X. Leng and Q. Shen, Angew. Chem. Int. Ed., 2015, 54, 7648; (c) B. Xing, C. Ni and J. Hu, Angew. Chem. Int. Ed., 2018, 57, 9896; (d) F. A. Siqueira, J. G. Taylor and C. R. D. Correia, Tetrahedron Lett., 2010, 51, 2102.

2. Z. Xia, J. Huang, Y. He, J. Zhao, J. Lei and Q. Zhu, Org. Lett., 2014, 16, 2546.

3. (a) X. Yan, S. Zhang, P. Zhang, X. Wu, A. Liu, G. Guo, Y. Dong and X. Li, Angew. Chem. Int. Ed., 2018, 57, 8947; (b) S. Asaoka, A. Joza, S. Minagawa, L. Song, Y. Suzuki and T. lyoda, ACS Macro Letters, 2013, 2, 906.

4. (a) N. B. Patel and J. C. Patel, J. Heterocycl. Chem., 2010, 47, 923; (b) N. P. Grimster, S. Connelly, A. Baranczak, J. Dong, L. B. Krasnova, K. B. Sharpless, E. T. Powers, I. A. Wilson and J. W. Kelly, J. Am. Chem. Soc., 2013, 135, 5656.

5. (a) D. Zheng, J. Yu and J. Wu, Angew. Chem. Int. Ed., 2016, 55, 11925; (b) T. Liu, W. Zhou and J. Wu, Org. Lett., $2017,19,6638$.

6. A. T. Davies, J. M. Curto, S. W. Bagley and M. C. Willis, Chem Sci, 2017, 8, 1233.

7. G. Laudadio, A. A. Bartolomeu, L. Verwijlen, Y. Cao, K. T. de Oliveira and T. Noel, J. Am. Chem. Soc., $2019,141,11832$.

8. Y. Liu, D. Yu, Y. Guo, J.-C. Xiao, Q.-Y. Chen and C. Liu, Org. Lett., 2020, 22, 2281.

9. L. Tang, Y. Yang, L. Wen, X. Yang and Z. Wang, Green Chem., 2016, 18, 1224.

10. A. L. Tribby, I. Rodriguez, S. Shariffudin and N. D. Ball, J Org Chem, 2017, 82, 2294.

11. H. Mukherjee, J. Debreczeni, J. Breed, S. Tentarelli, B. Aquila, J. E. Dowling, A. Whitty and N. P. Grimster, Org. Biomol. Chem., $2017,15,9685$.

12. A. Talko, D. Antoniak and M. Barbasiewicz, Synthesis, 2019, 51, 2278.

13. Q. Zheng, J. L. Woehl, S. Kitamura, D. Santos-Martins, C. J. Smedley, G. Li, S. Forli, J. E. Moses, D. W. Wolan and K. B. Sharpless, Proc Natl Acad Sci U $S A, 2019,116,18808$

14. J.-M. Li, Y.-H. Wang, Y. Yu, R.-B. Wu, J. Weng and G. Lu, ACS Catal., 2017, 7, 2661.

15. R. Gardi, R. Vitali, G. Falconi and A. Ercoli, J. Med. Chem., 1973, 16, 123. 
11. ${ }^{1} \mathrm{H},{ }^{13} \mathrm{C}$ and ${ }^{19} \mathrm{~F}$ NMR Spectra of Compounds 2a-2z, 2aa-2ai, 2ak and 3aj, s1-s4

${ }^{1} \mathrm{H}$ NMR (400 MHz, $\left.\mathrm{CDCl}_{3}\right)$

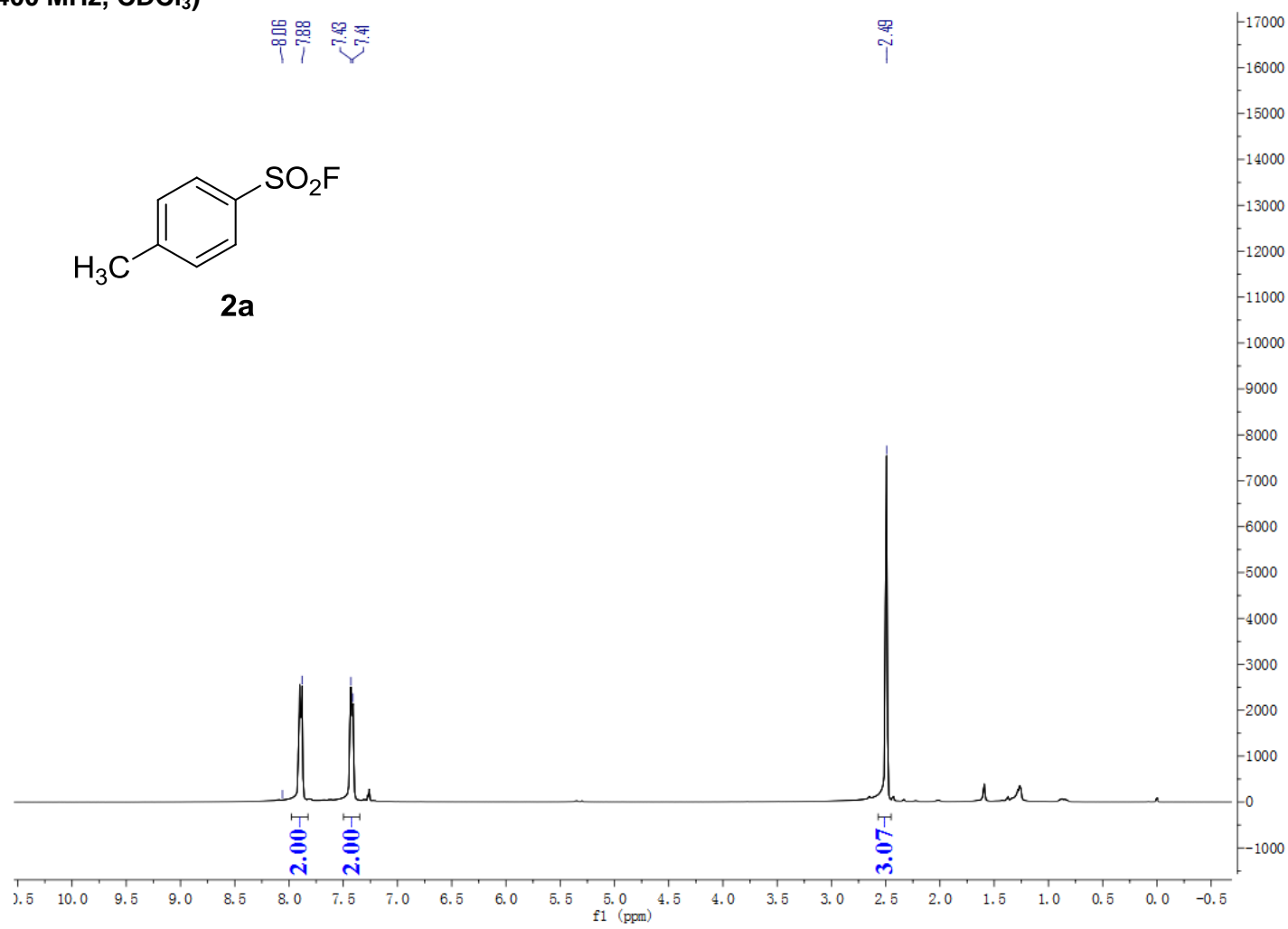

${ }^{13} \mathrm{C}$ NMR (101 MHz, $\left.\mathrm{CDCl}_{3}\right)$

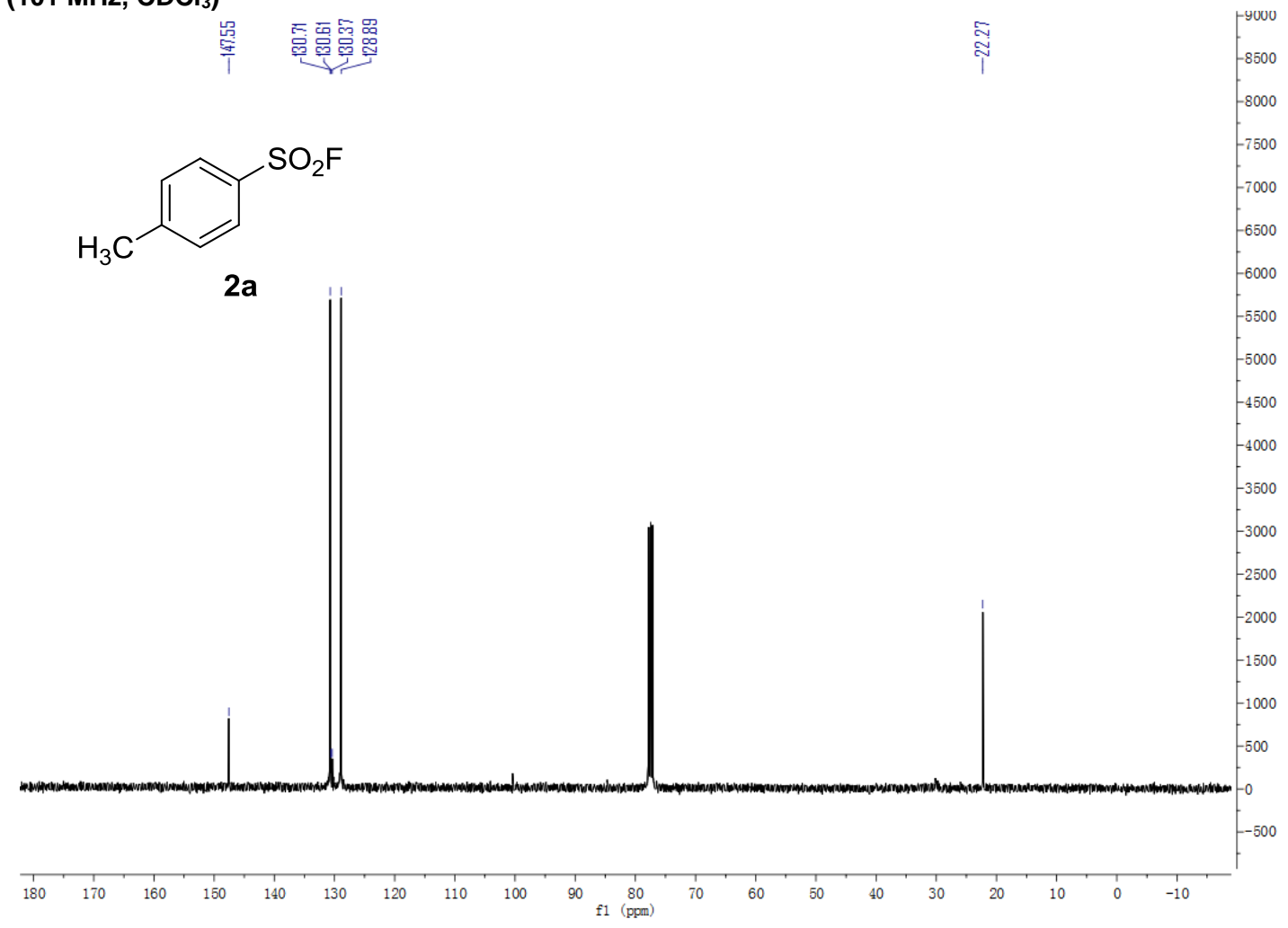


${ }^{19} \mathrm{~F} \mathrm{NMR} \mathrm{(376} \mathrm{MHz,} \mathrm{CDCl}_{3}$ )

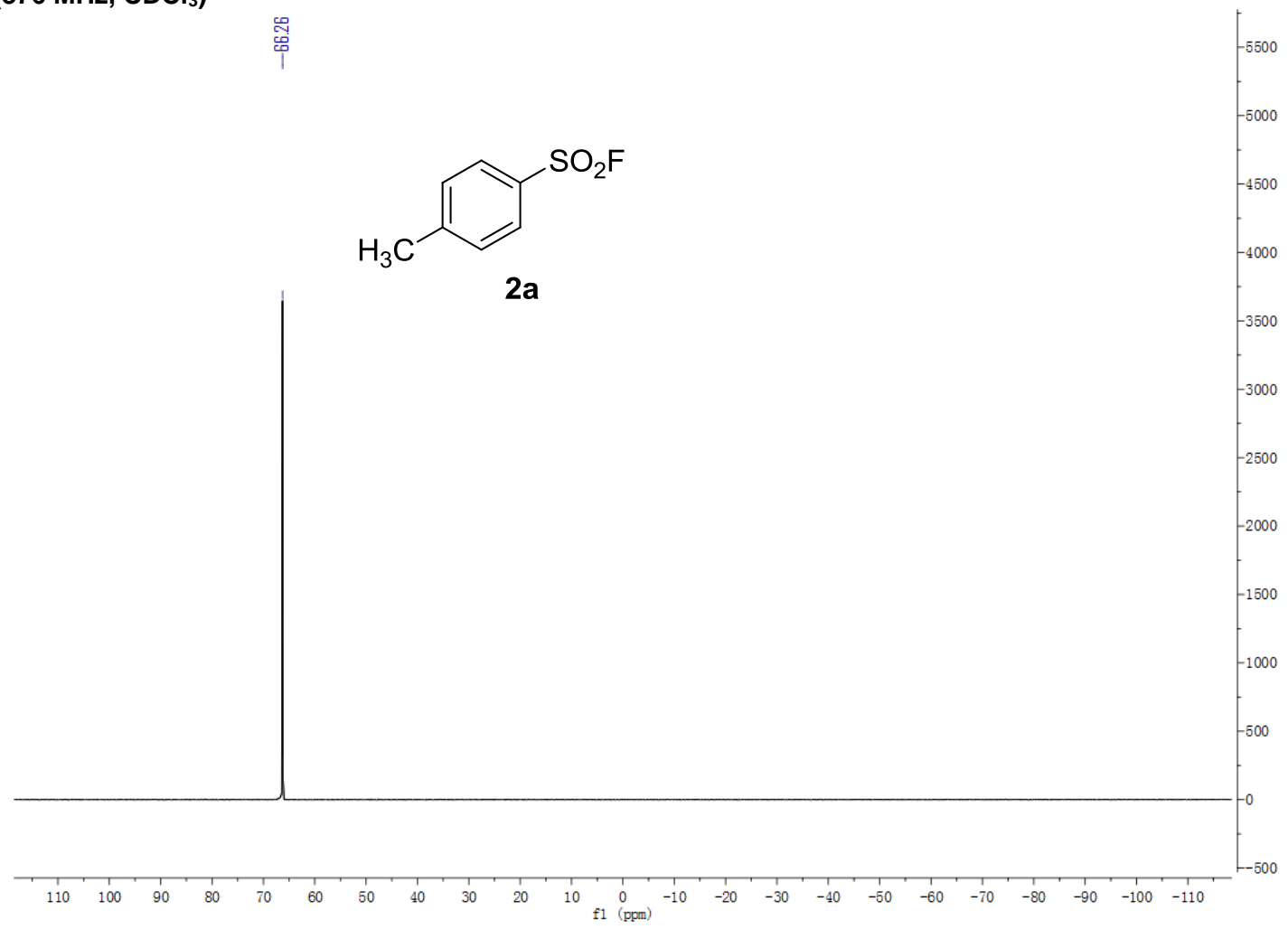

${ }^{1} \mathrm{H}$ NMR (400 MHz, $\left.\mathrm{CDCl}_{3}\right)$

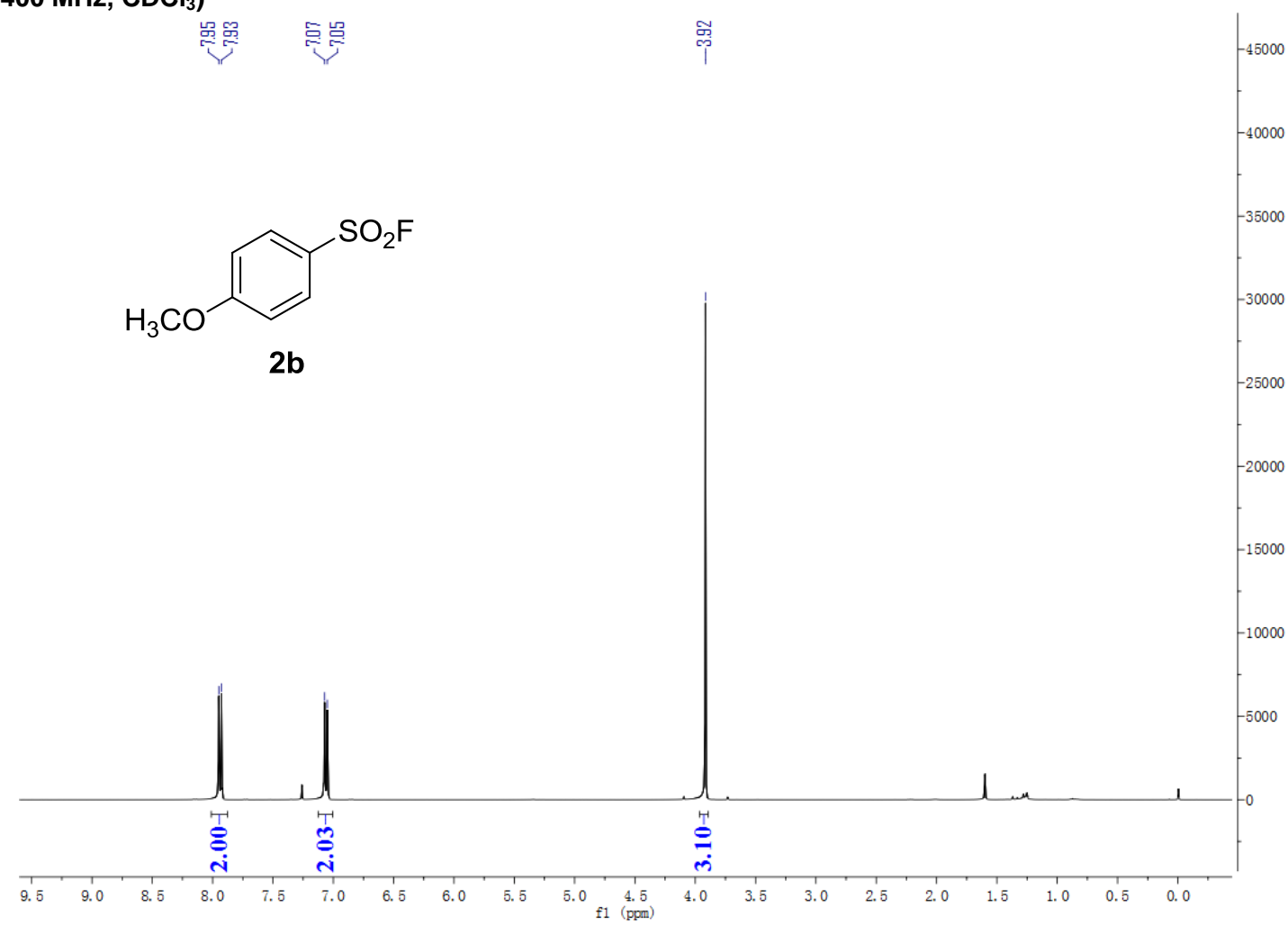


${ }^{13} \mathrm{C}$ NMR (101 MHz, $\mathrm{CDCl}_{3}$ )

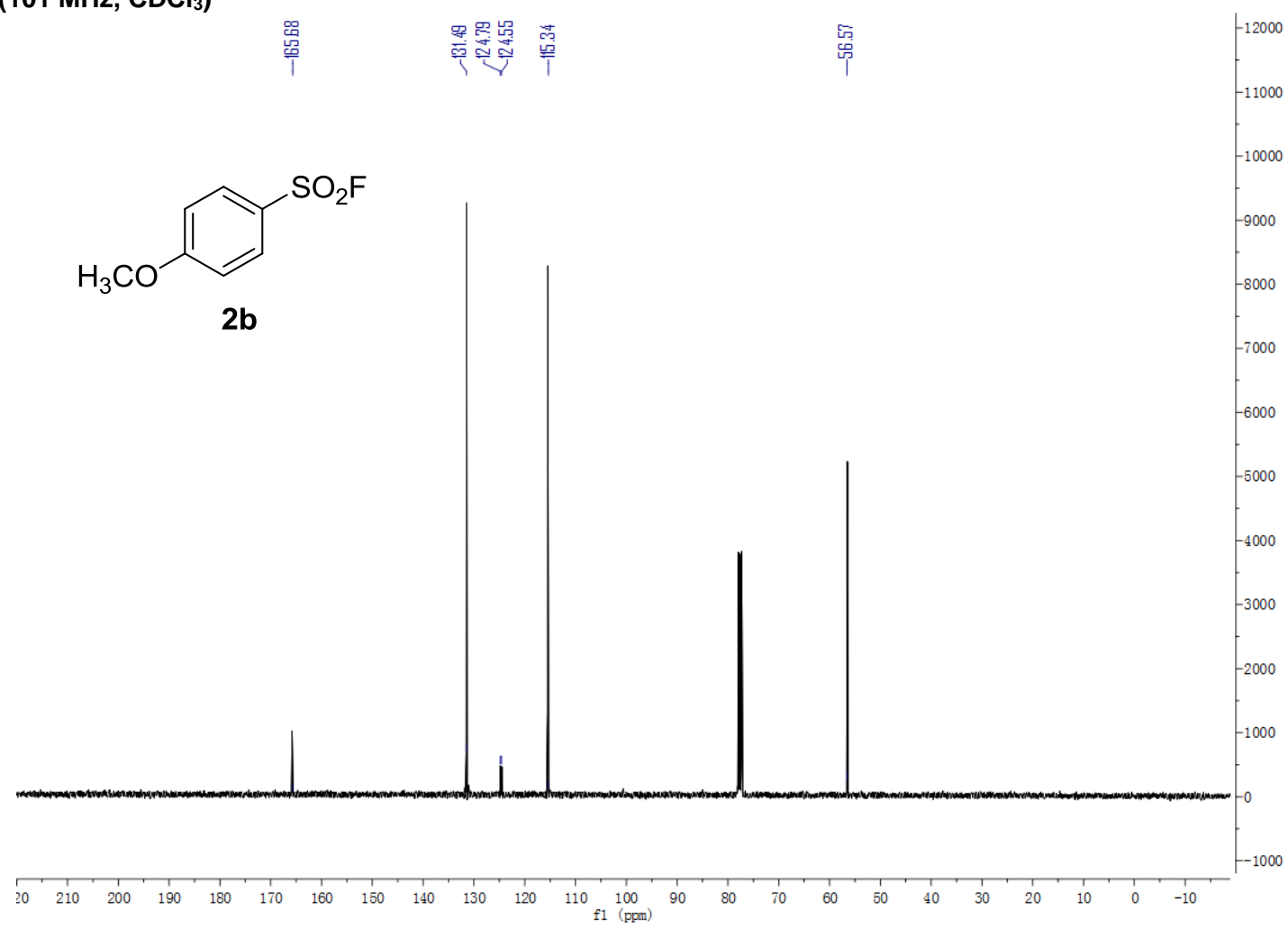

${ }^{19} \mathrm{~F}$ NMR (376 MHz, $\mathrm{CDCl}_{3}$ )

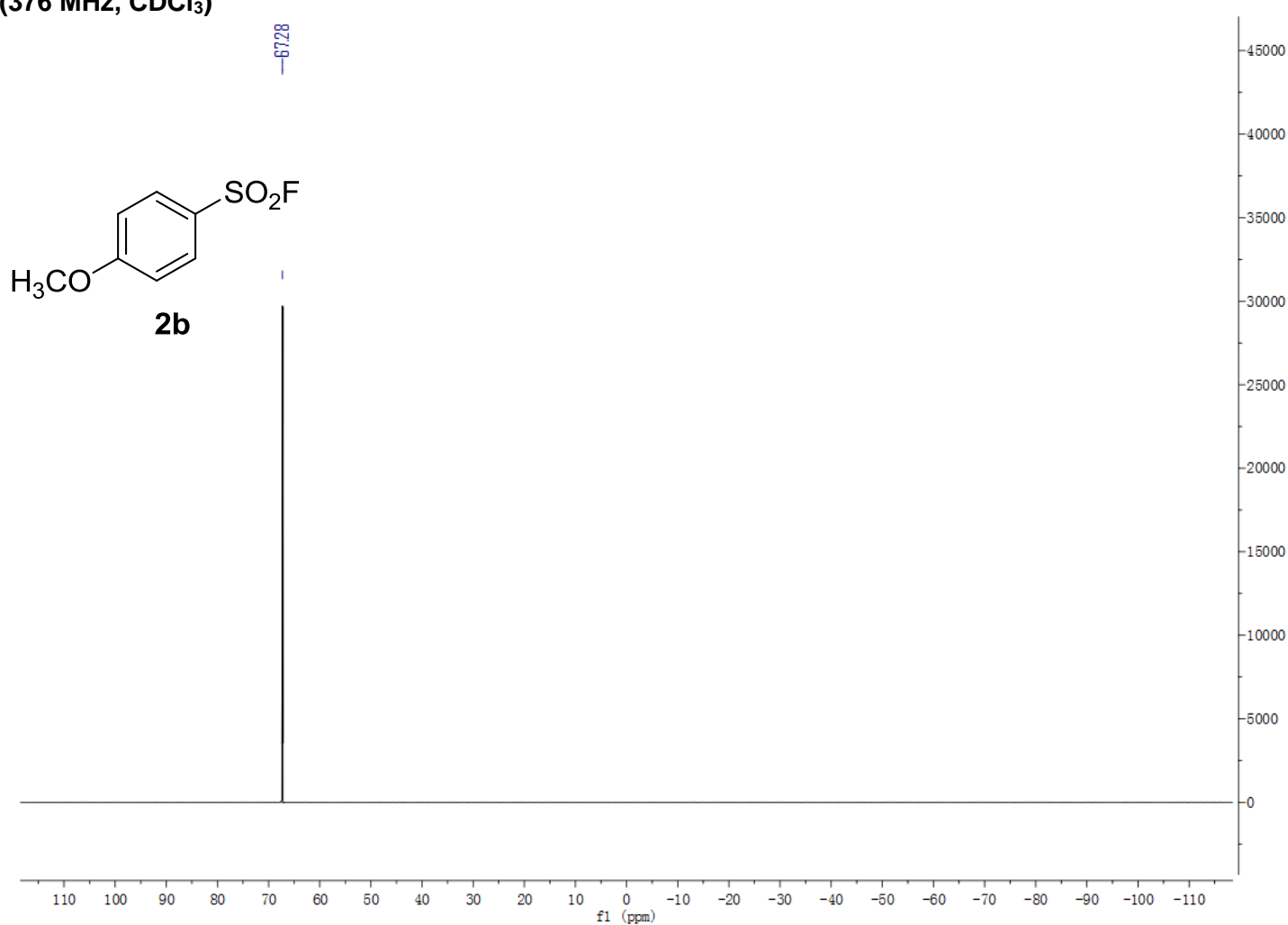


${ }^{1} \mathrm{H}$ NMR $\left(400 \mathrm{MHz}, \mathrm{CDCl}_{3}\right)$

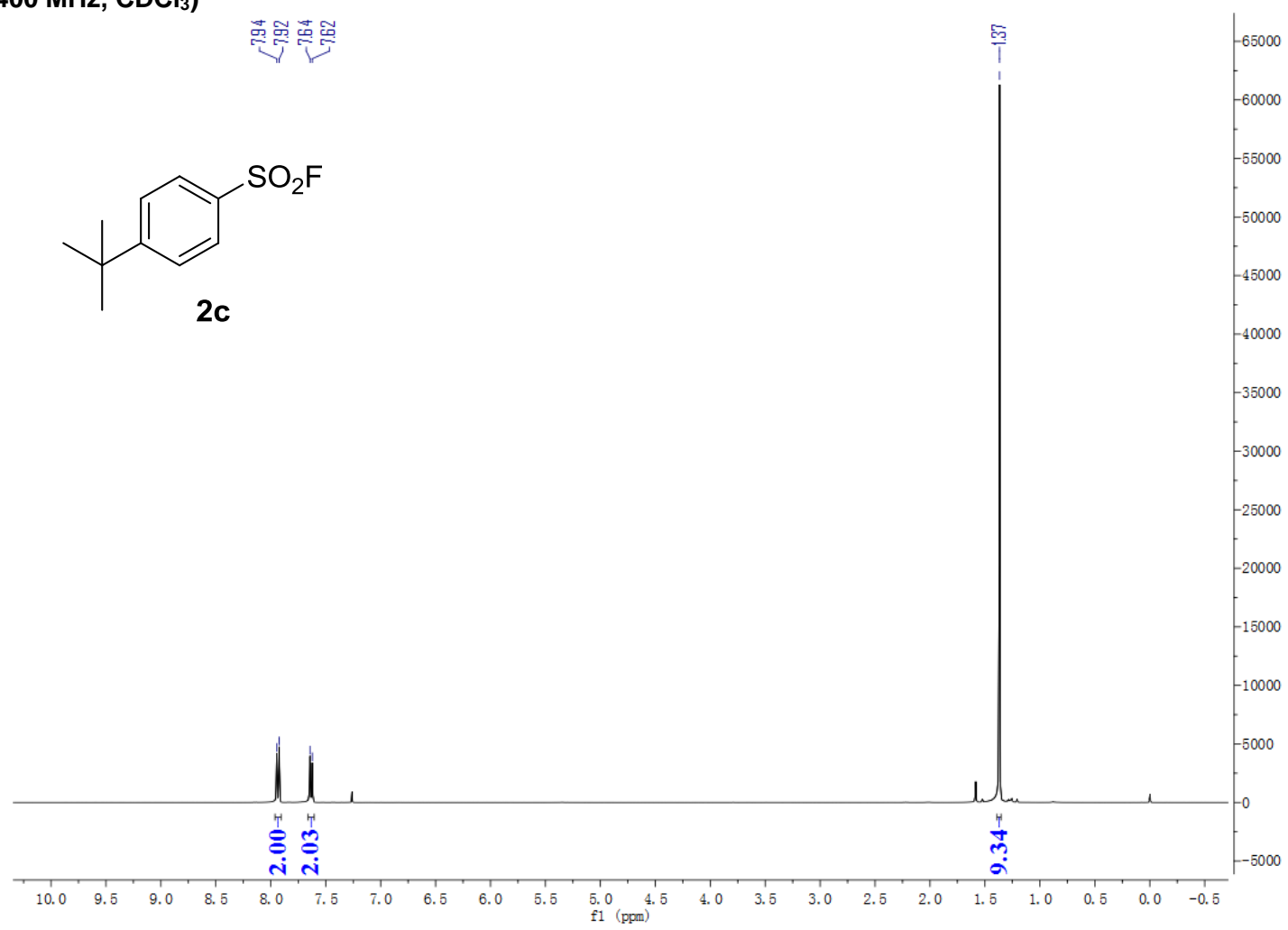

${ }^{13} \mathrm{C}$ NMR (101 MHz, $\left.\mathrm{CDCl}_{3}\right)$

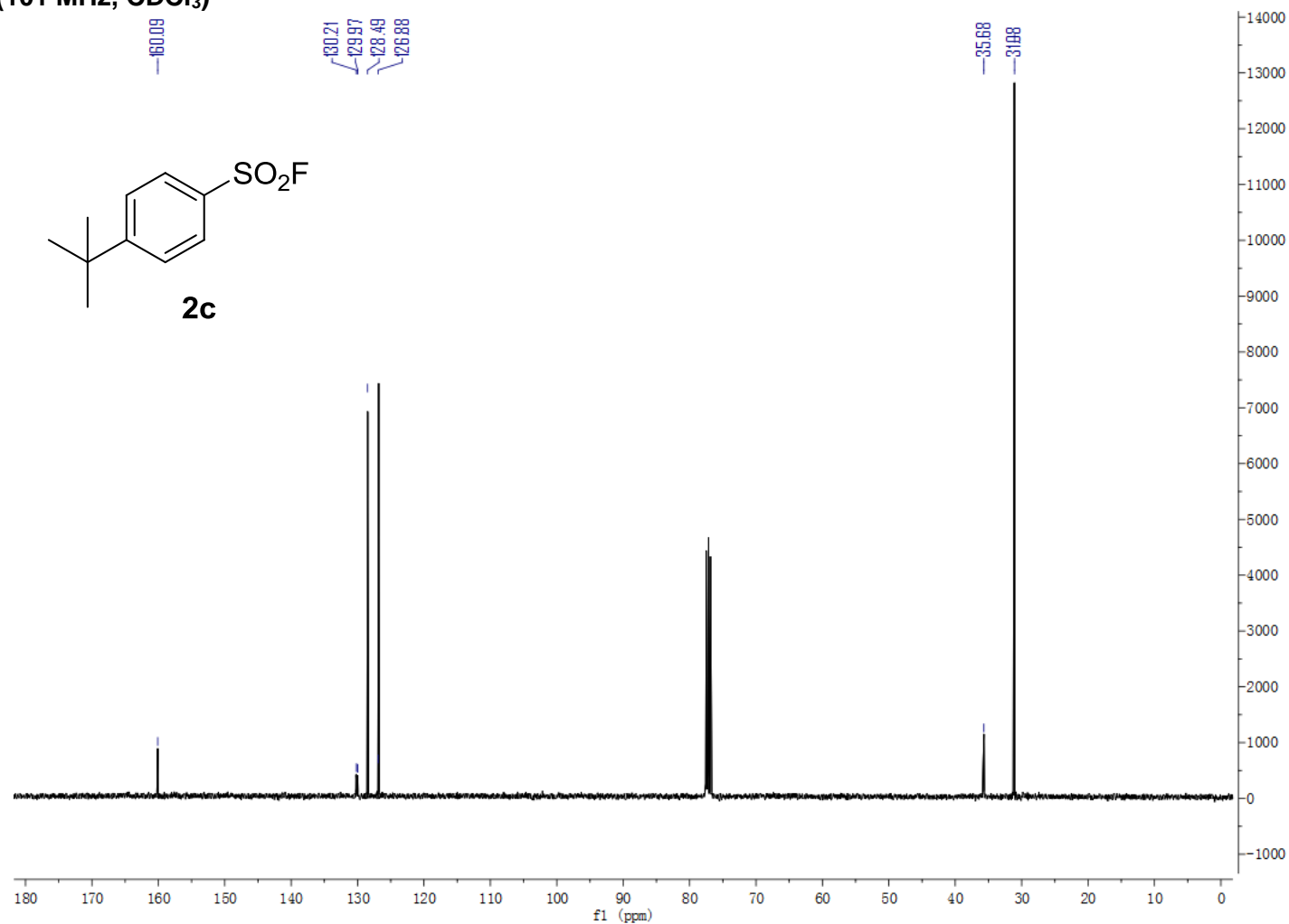


${ }^{19}$ F NMR (376 MHz, $\mathrm{CDCl}_{3}$ )

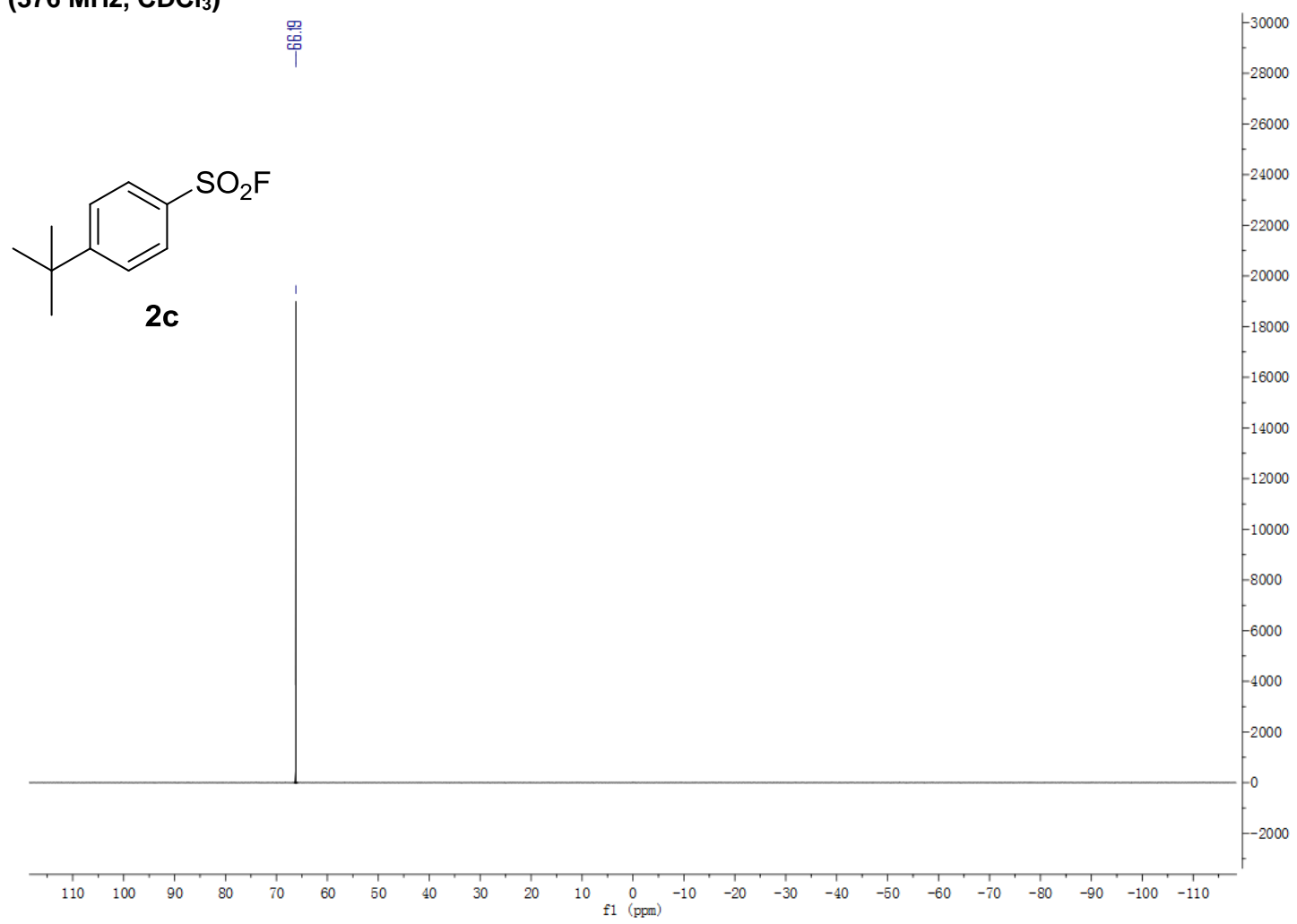

${ }^{1} \mathrm{H}$ NMR (400 MHz, $\mathrm{CDCl}_{3}$ )

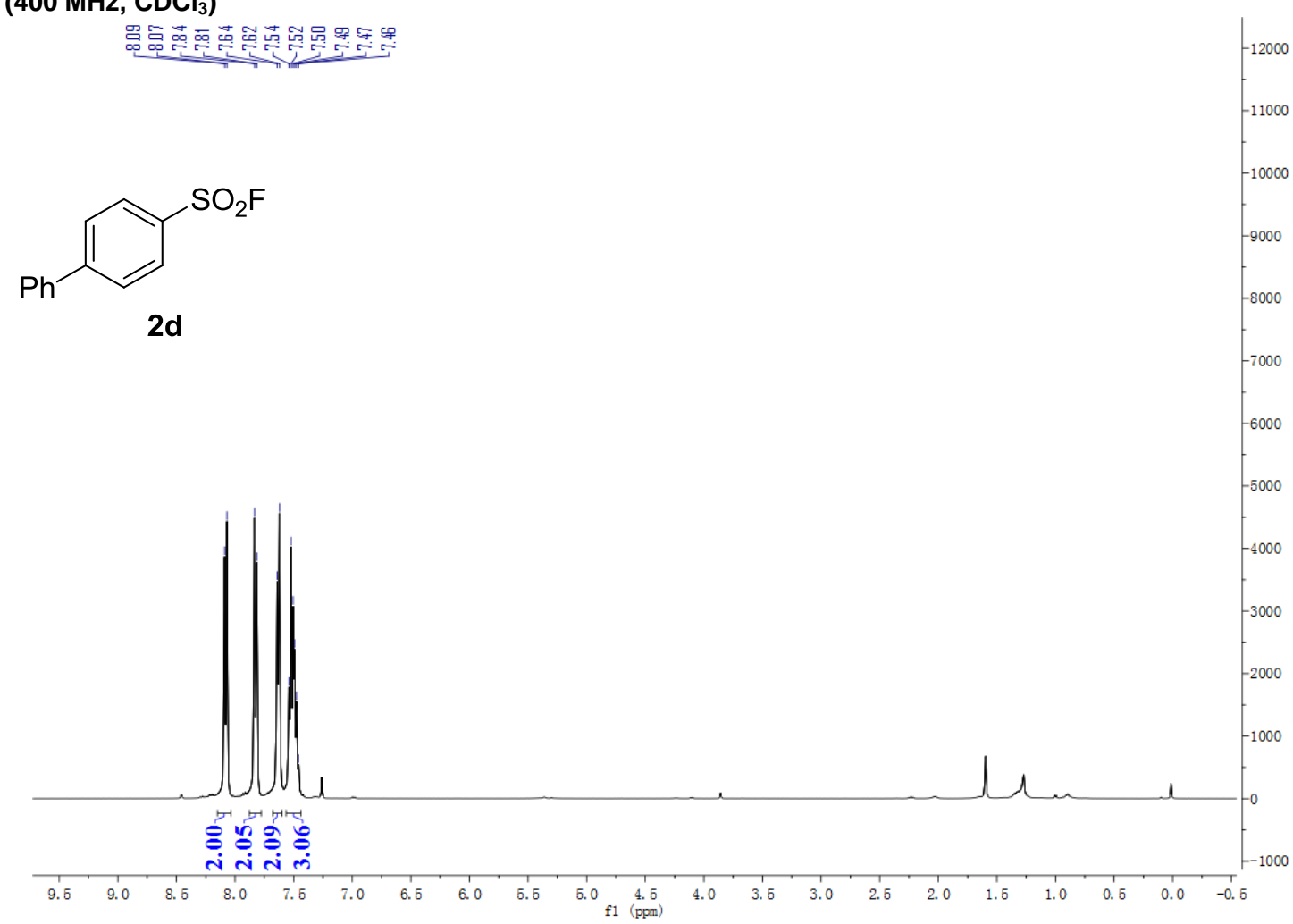


${ }^{13} \mathrm{C}$ NMR (126 MHz, $\mathrm{CDCl}_{3}$ )

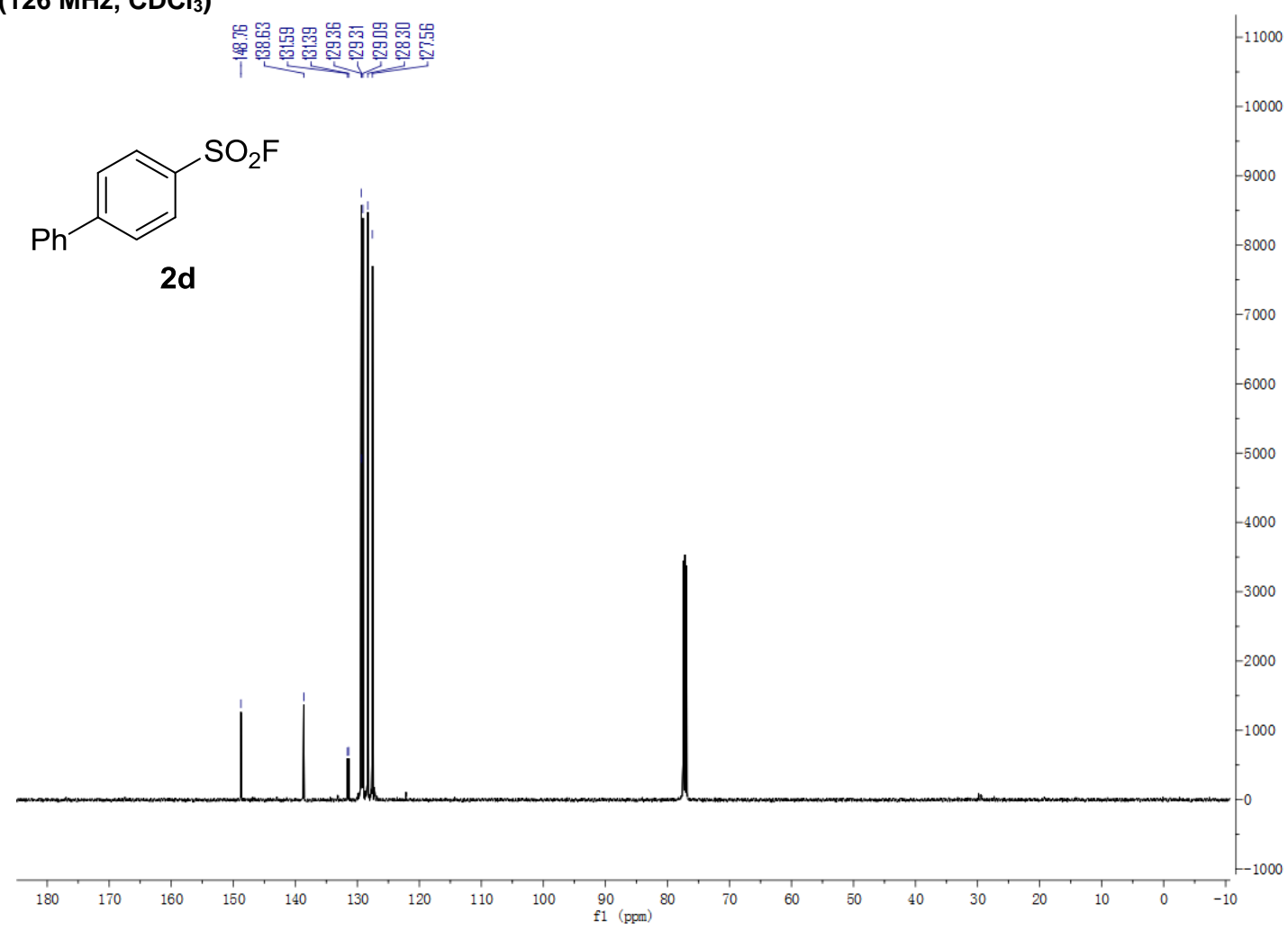

${ }^{19} \mathrm{~F} \mathrm{NMR} \mathrm{(376} \mathrm{MHz,} \mathrm{CDCl}_{3}$ )

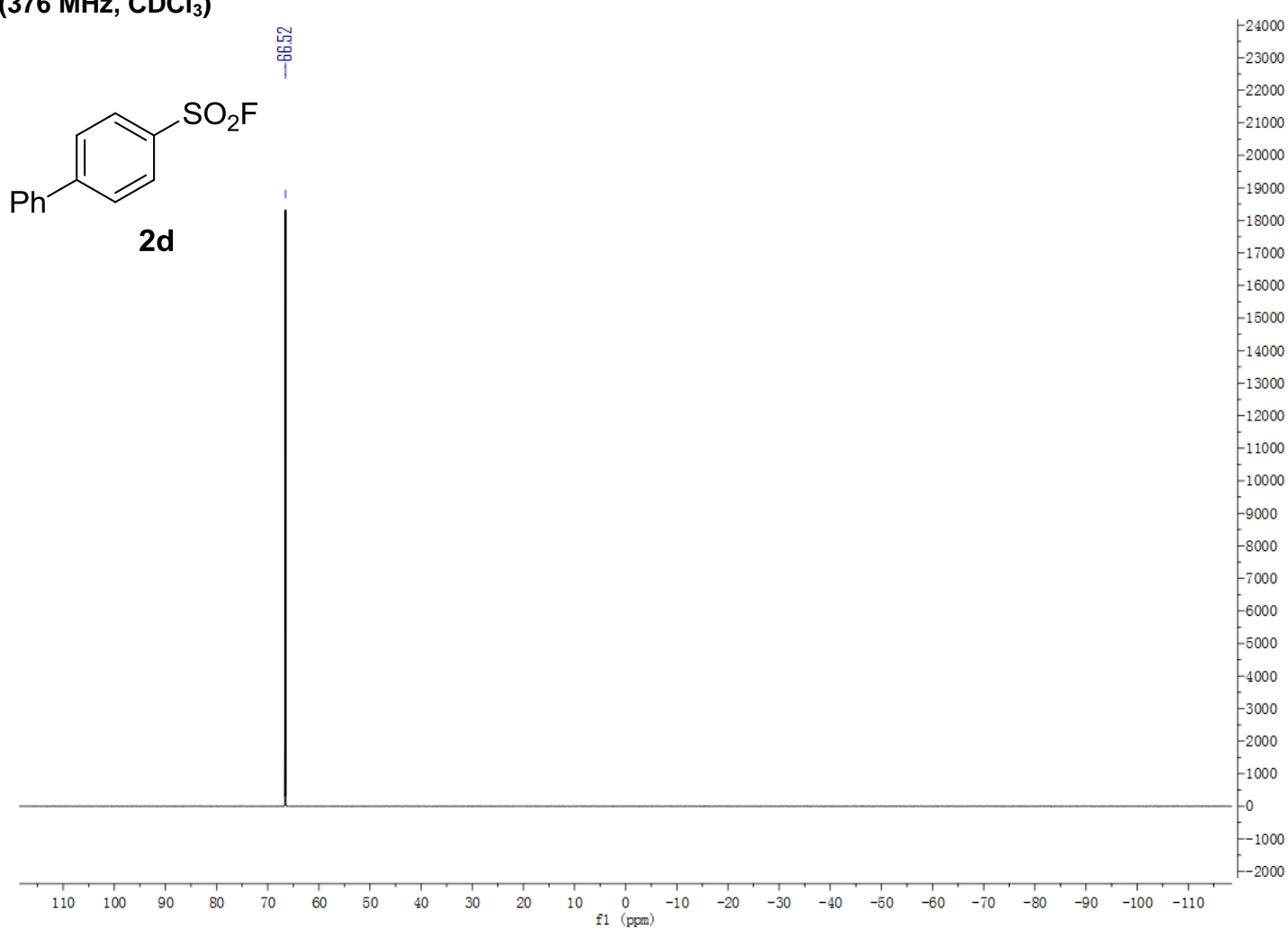


${ }^{1} \mathrm{H}$ NMR (400 MHz, $\mathrm{CDCl}_{3}$ )

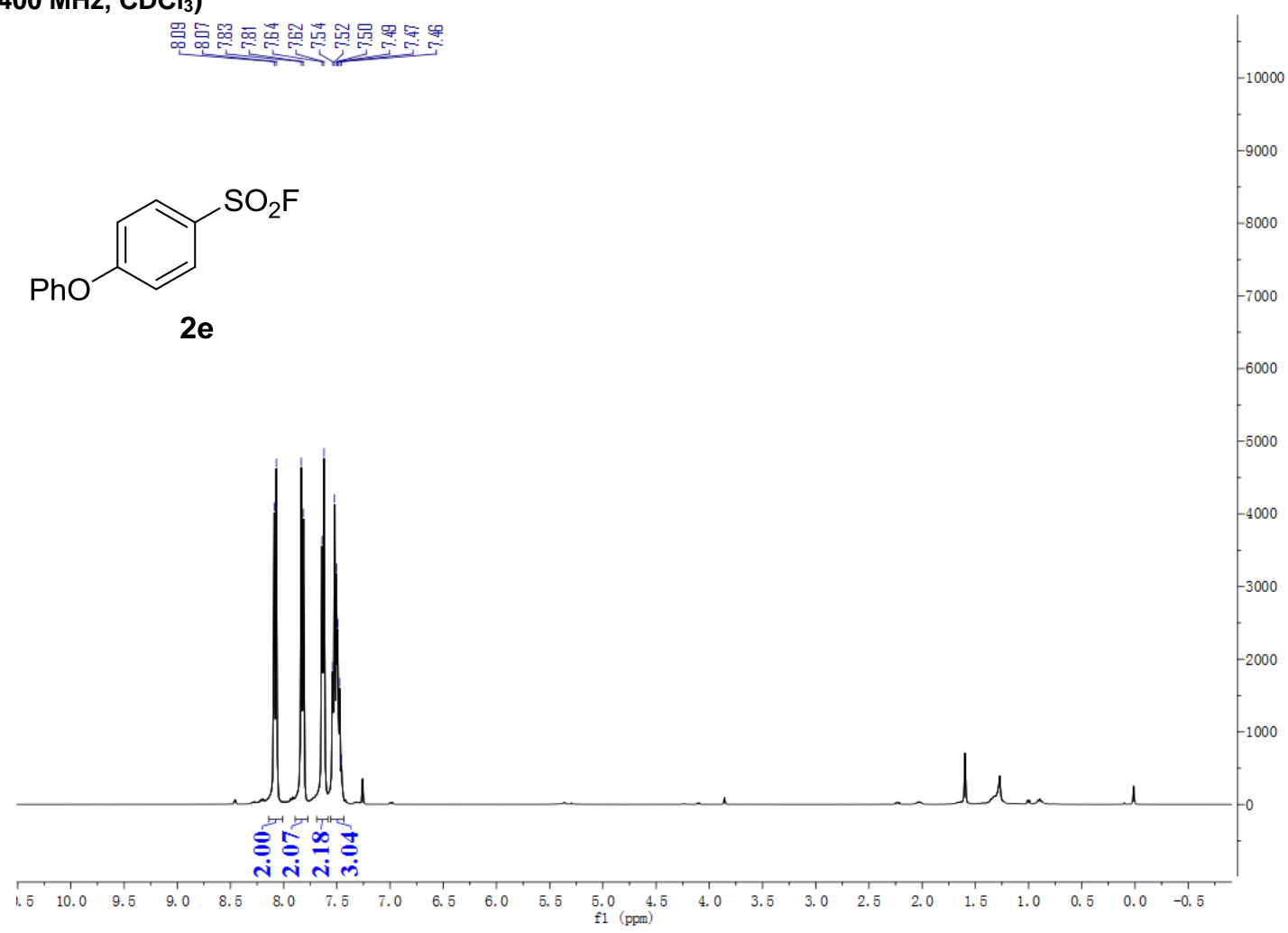

${ }^{13} \mathrm{C}$ NMR (101 MHz, $\mathrm{CDCl}_{3}$ )

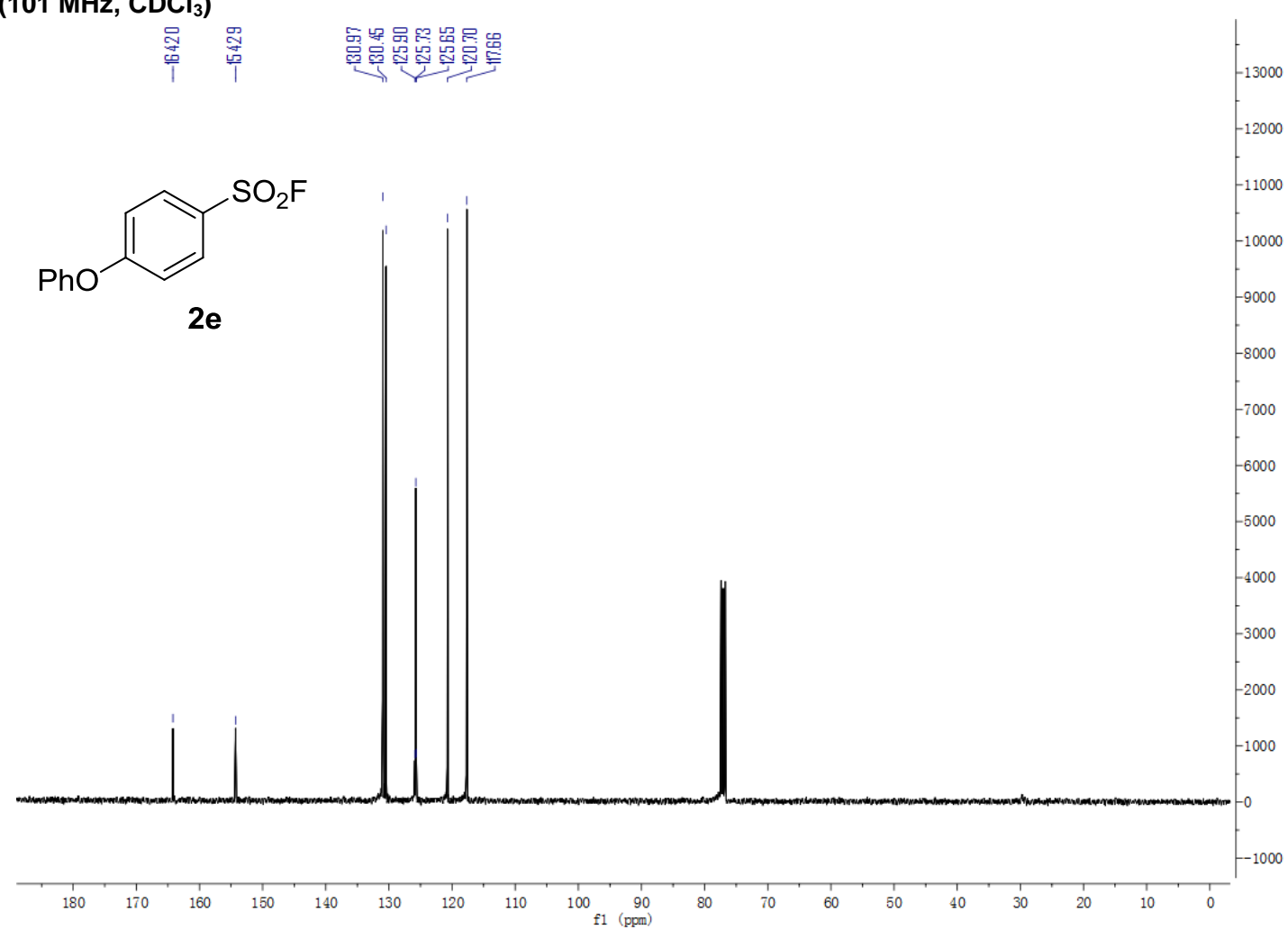


$\left.{ }^{19} \mathrm{~F} \mathrm{NMR} \mathrm{(376} \mathrm{MHz,} \mathrm{CDCl}_{3}\right)$

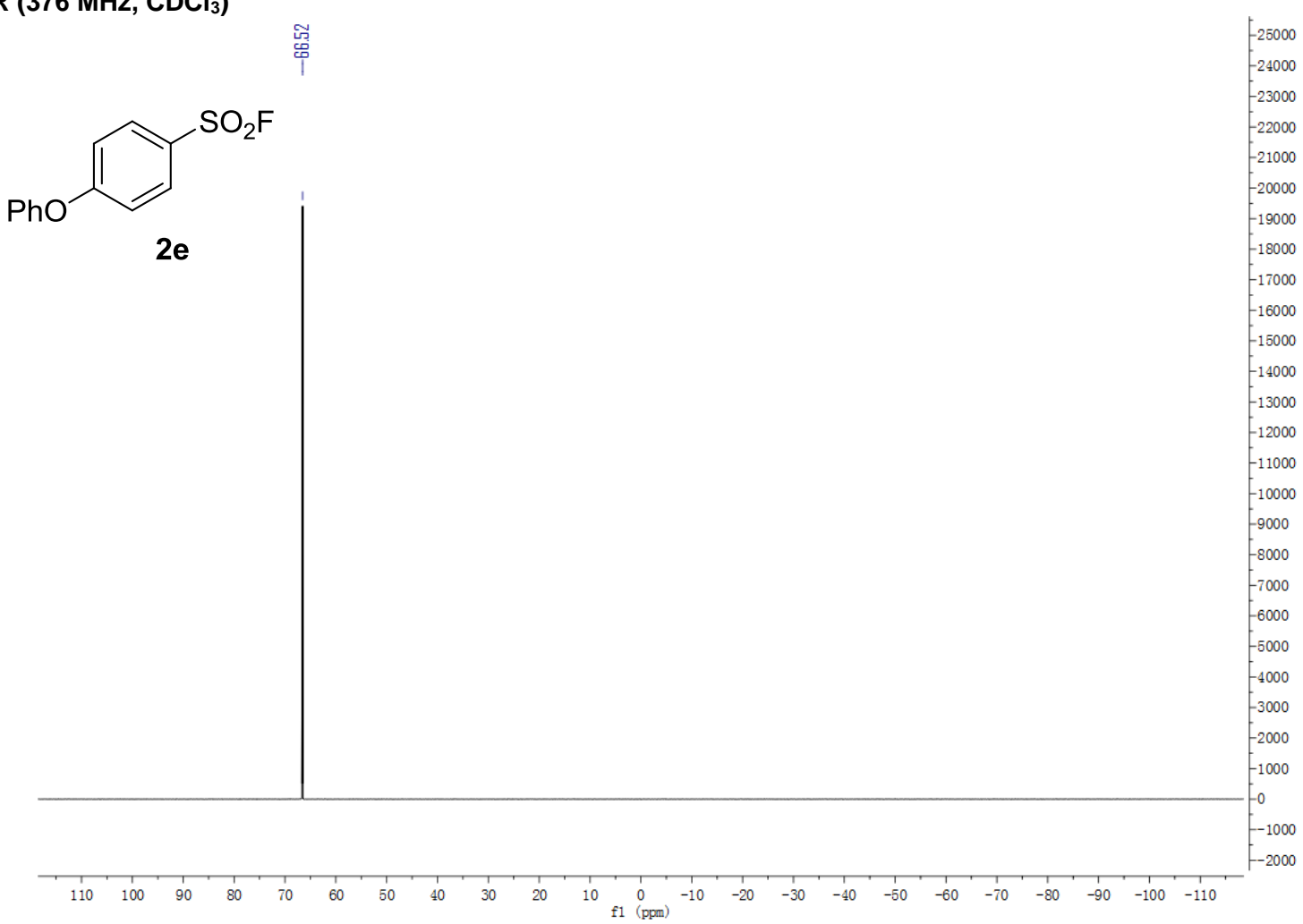

${ }^{1} \mathrm{H}$ NMR $\left(400 \mathrm{MHz}, \mathrm{CDCl}_{3}\right)$

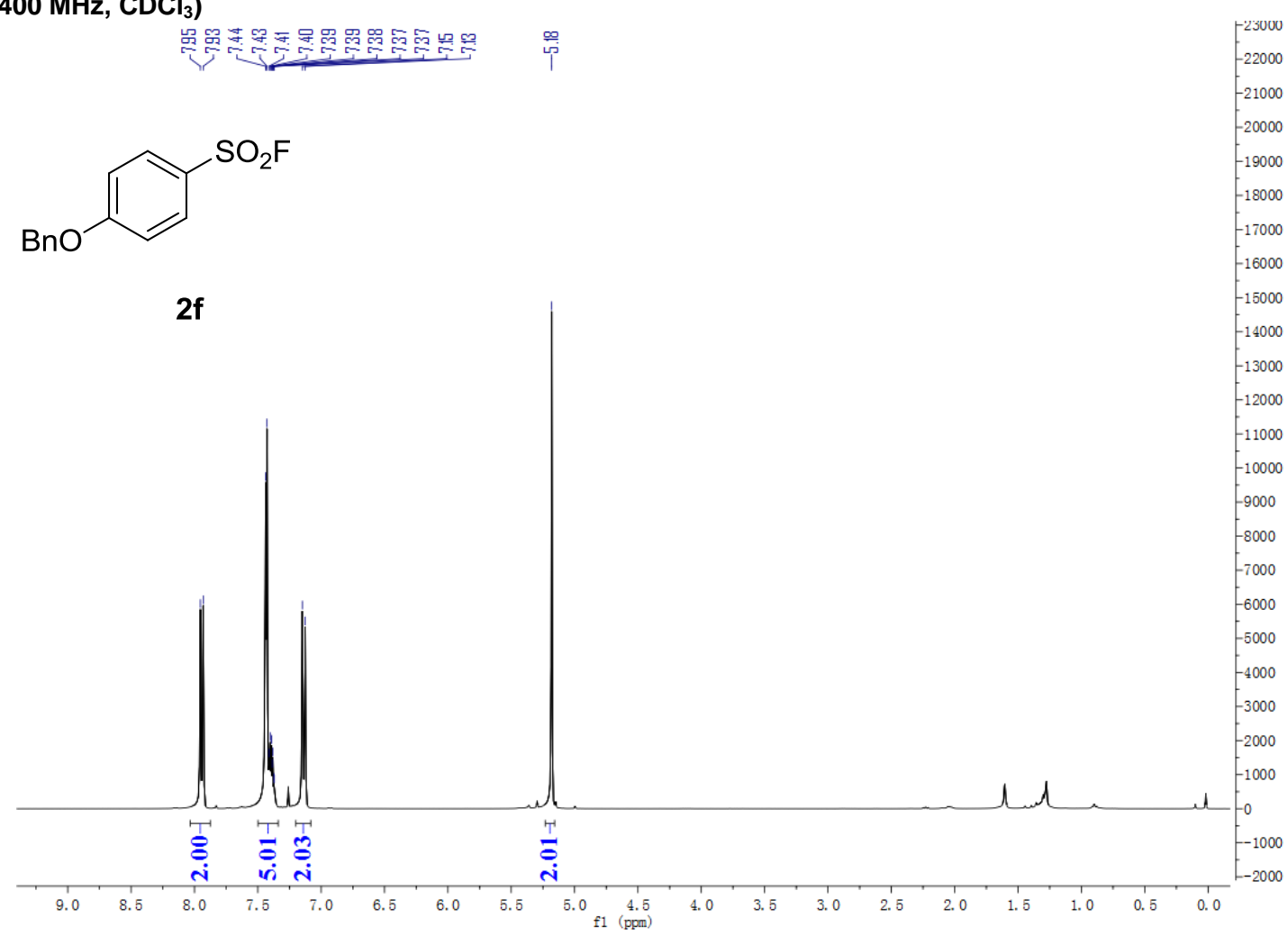


${ }^{13} \mathrm{C}$ NMR (101 MHz, $\mathrm{CDCl}_{3}$ )

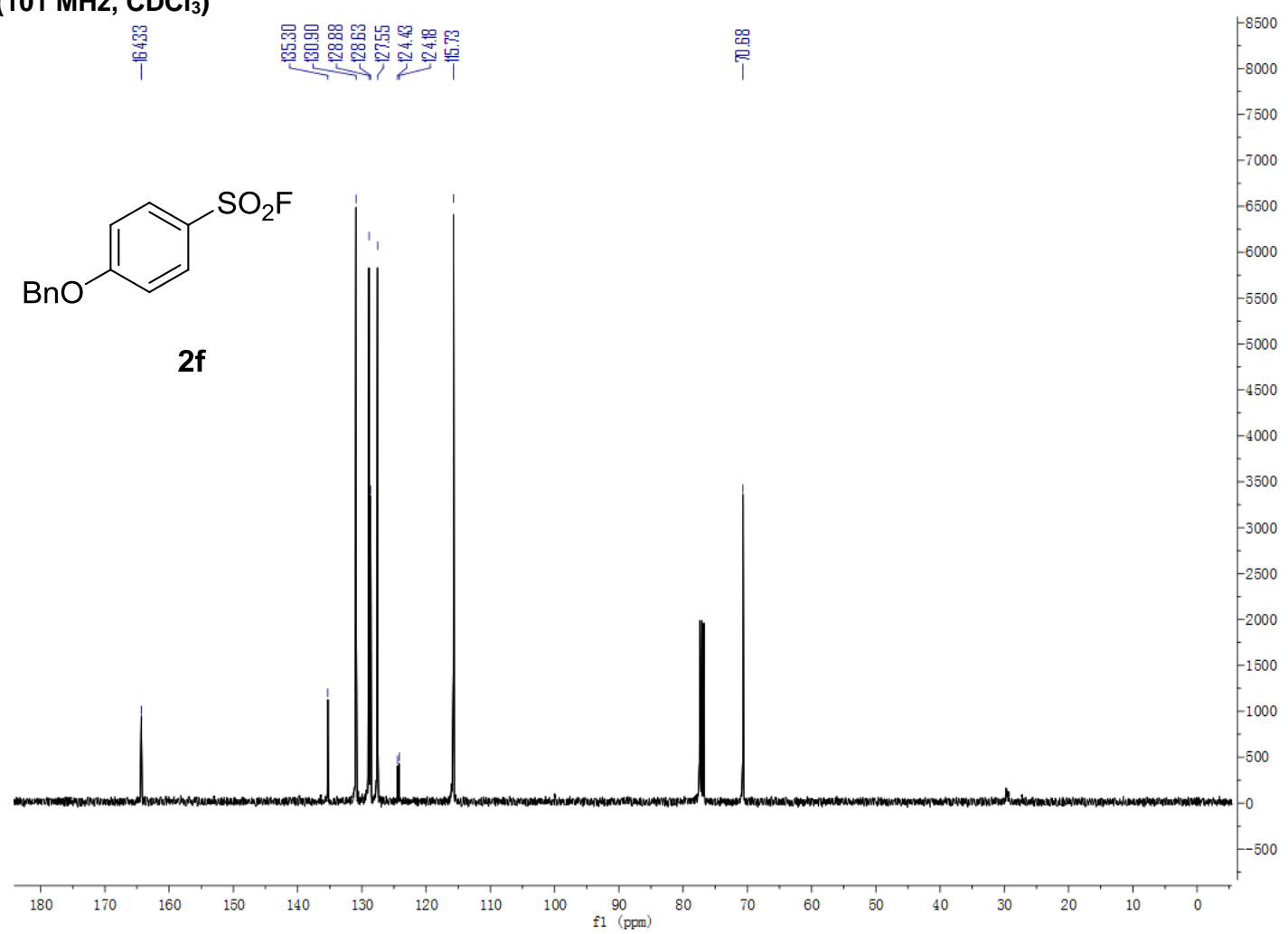

${ }^{19} \mathrm{~F}$ NMR (376 MHz, $\mathrm{CDCl}_{3}$ )

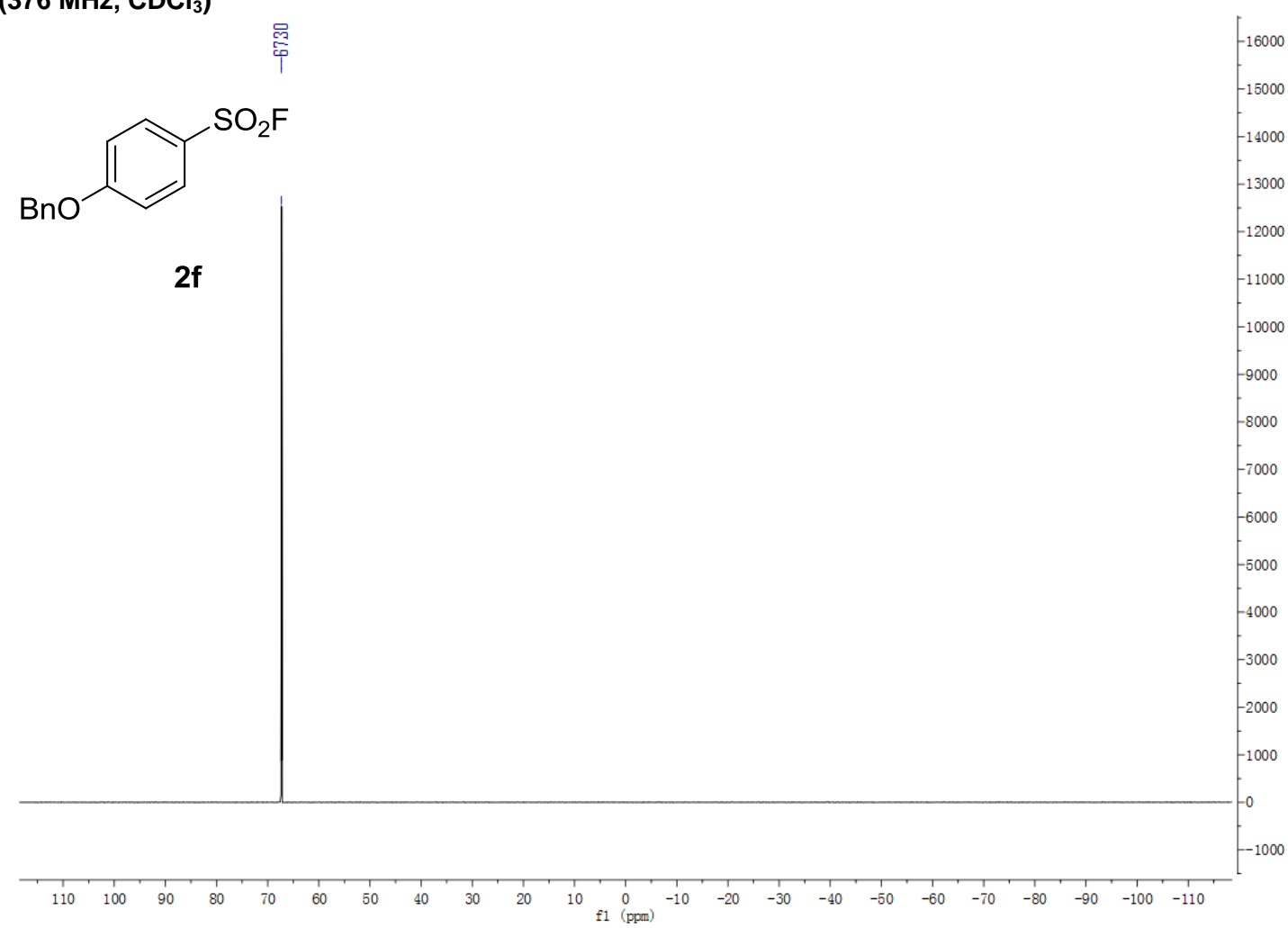


${ }^{1} \mathrm{H}$ NMR (400 MHz, DMSO- $\mathrm{d}_{6}$ )

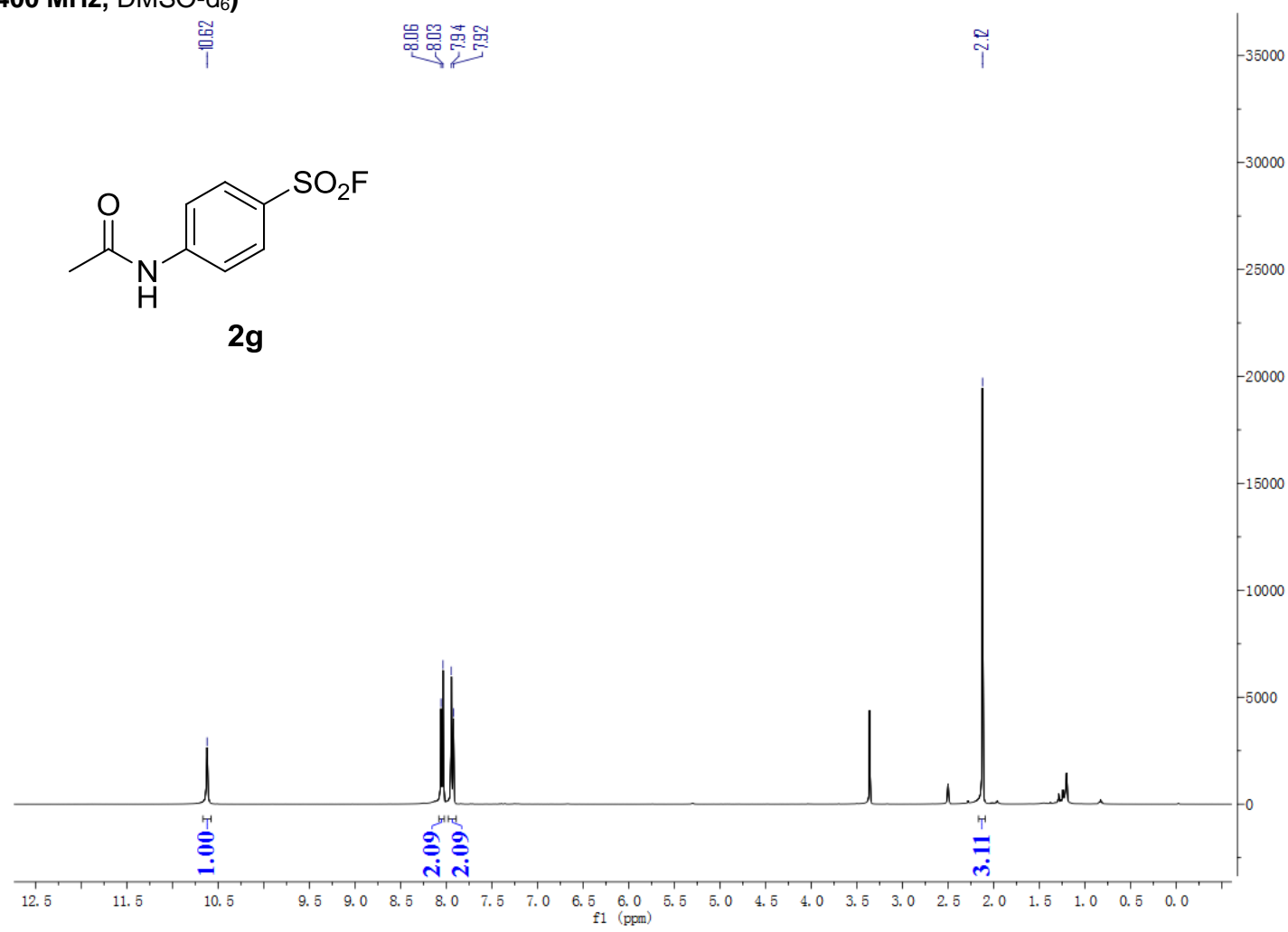

${ }^{13} \mathrm{C}$ NMR (101 MHz, DMSO-d 6 )

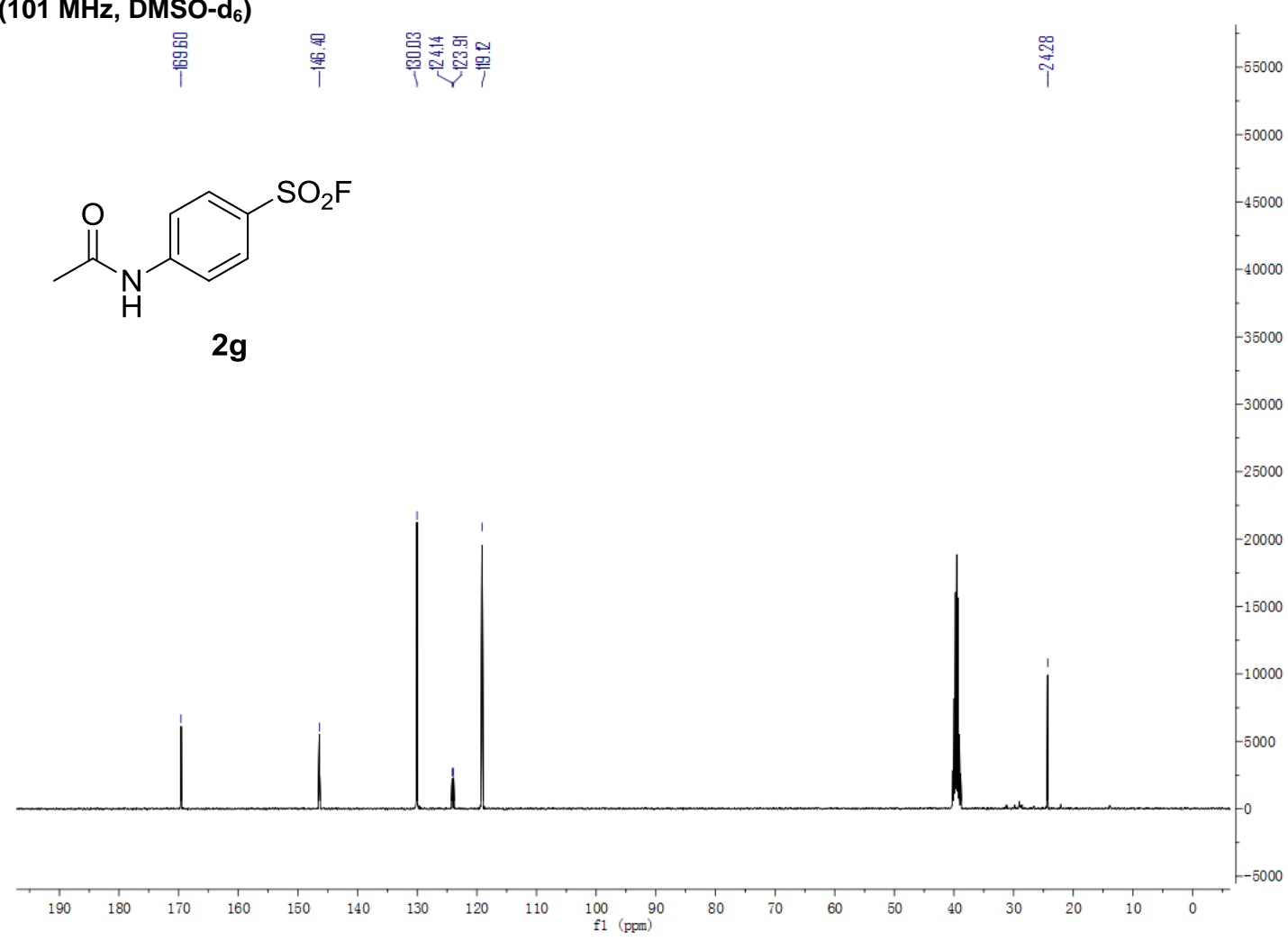


${ }^{19}$ F NMR (376 MHz, DMSO-d 6 )

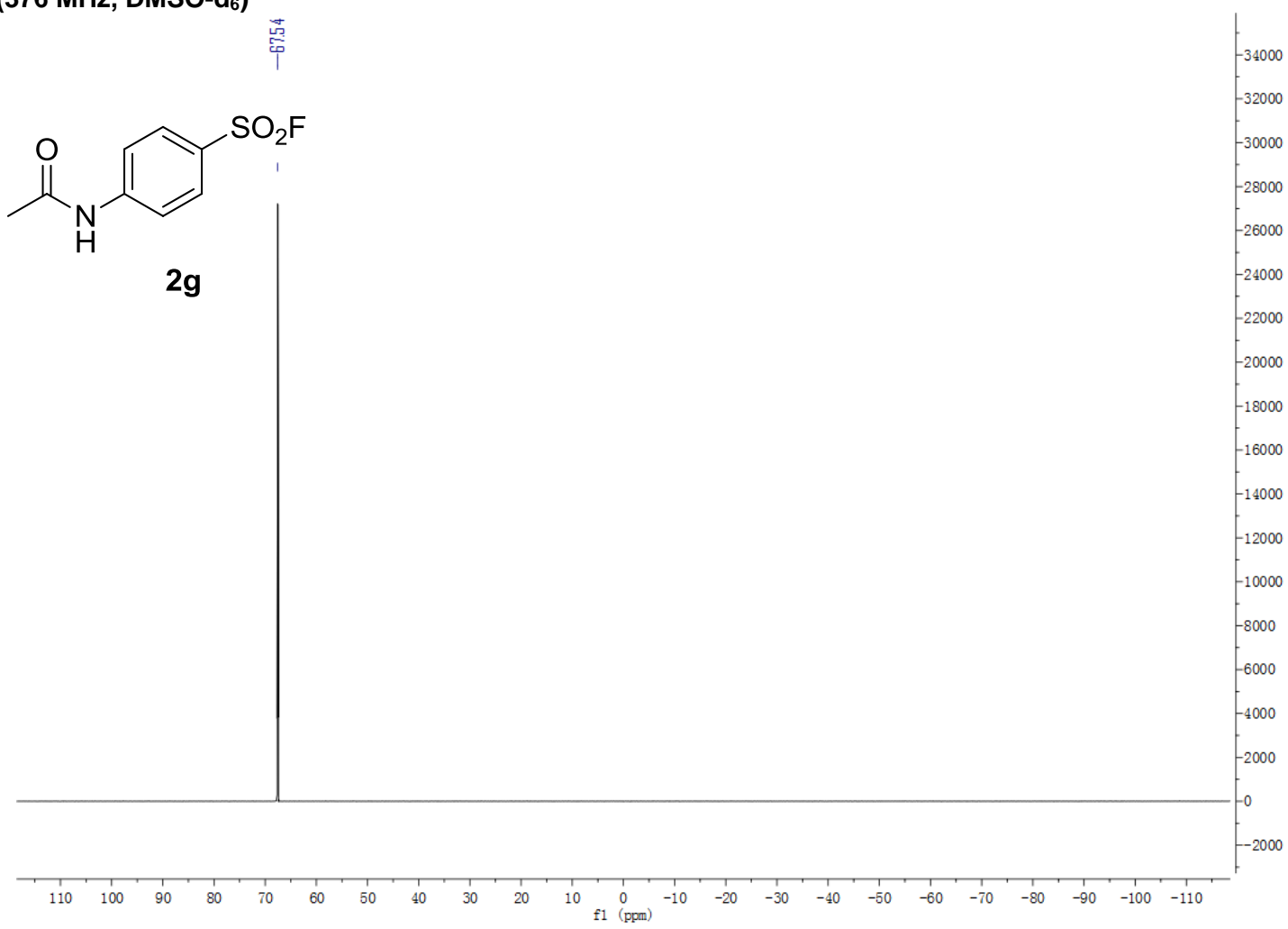

${ }^{1} \mathrm{H}$ NMR (400 MHz, $\mathrm{CDCl}_{3}$ )

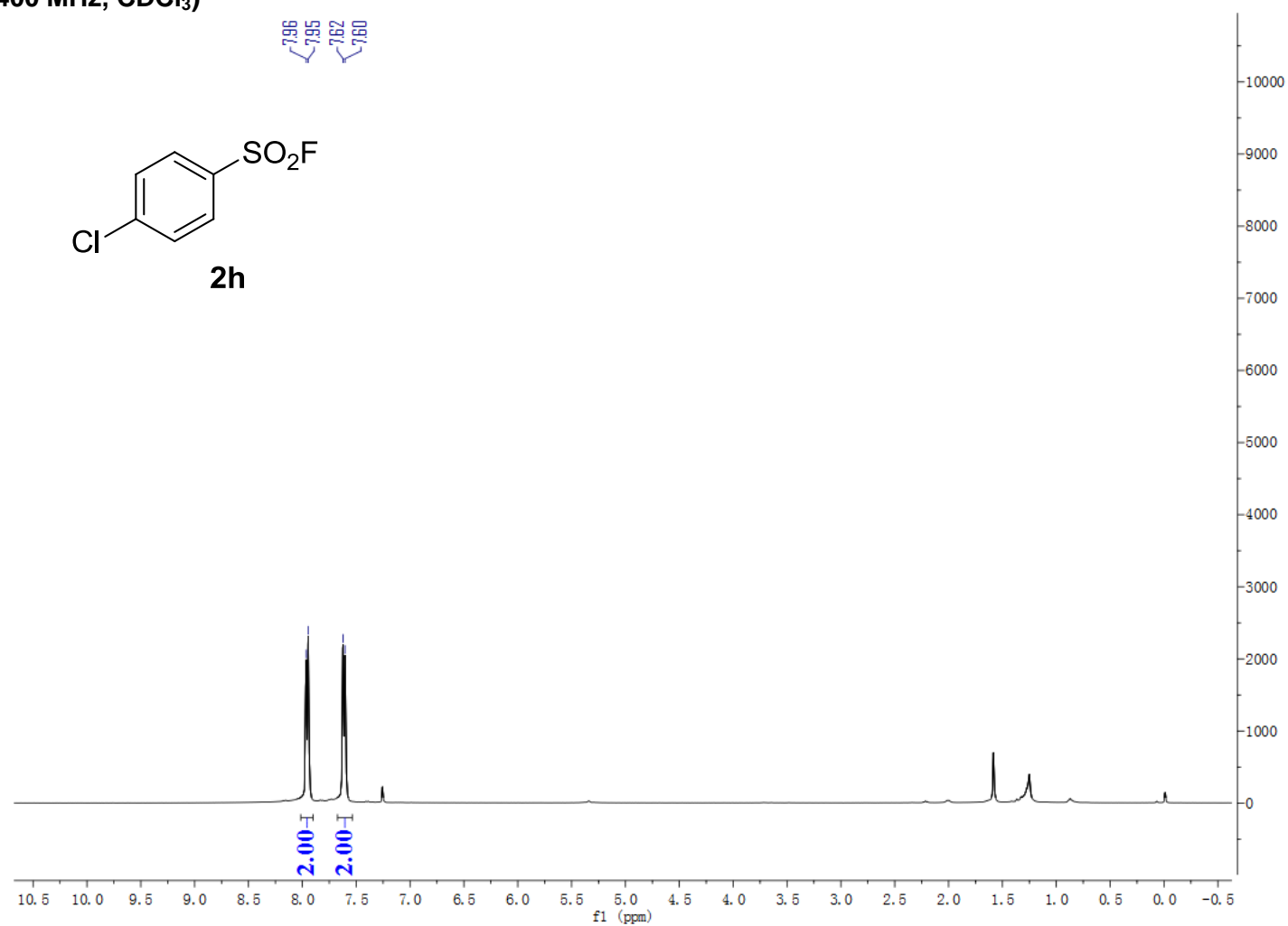


${ }^{13} \mathrm{C}$ NMR (101 MHz, $\mathrm{CDCl}_{3}$ )

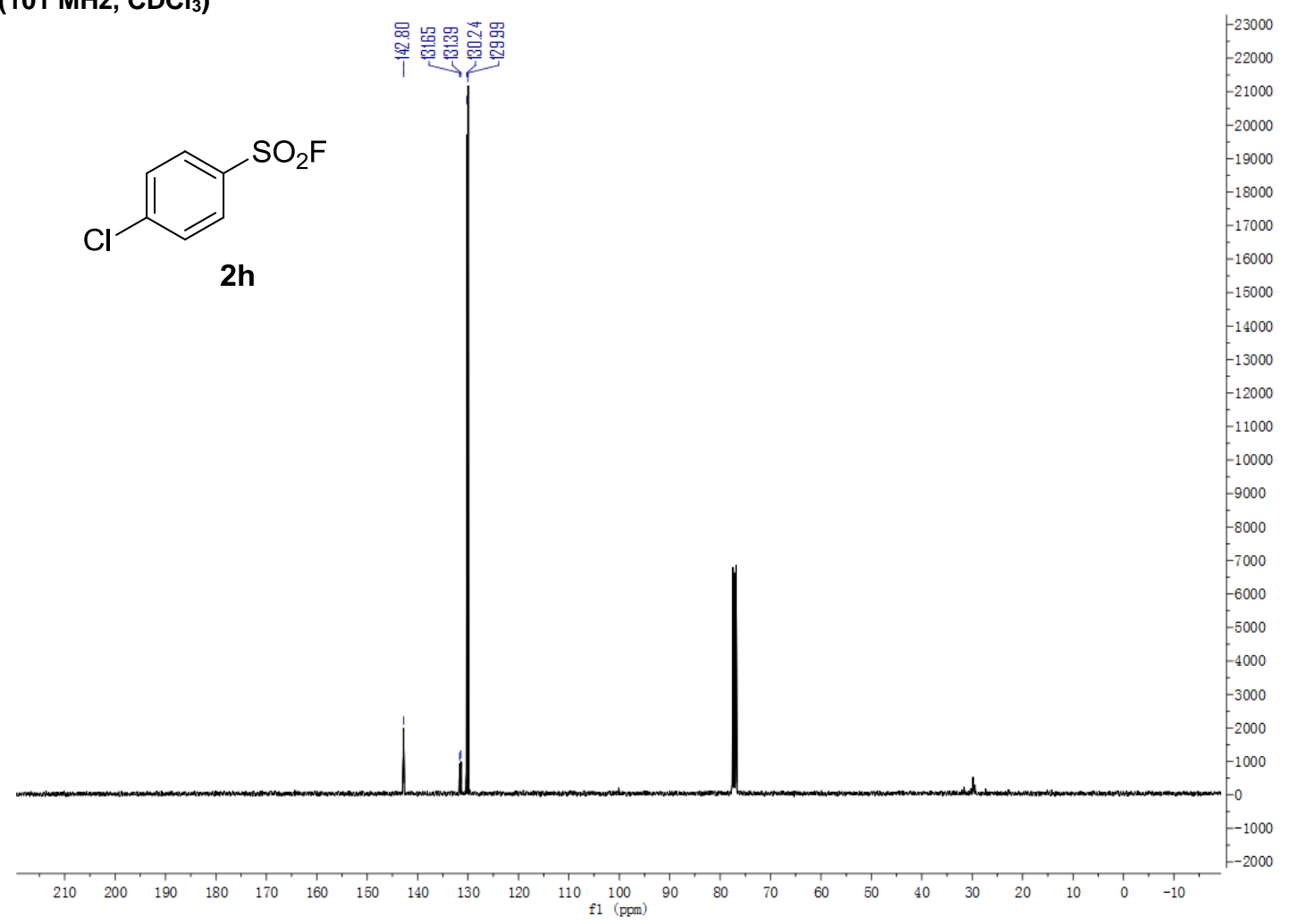

${ }^{19} \mathrm{~F}$ NMR (376 MHz, $\mathrm{CDCl}_{3}$ )

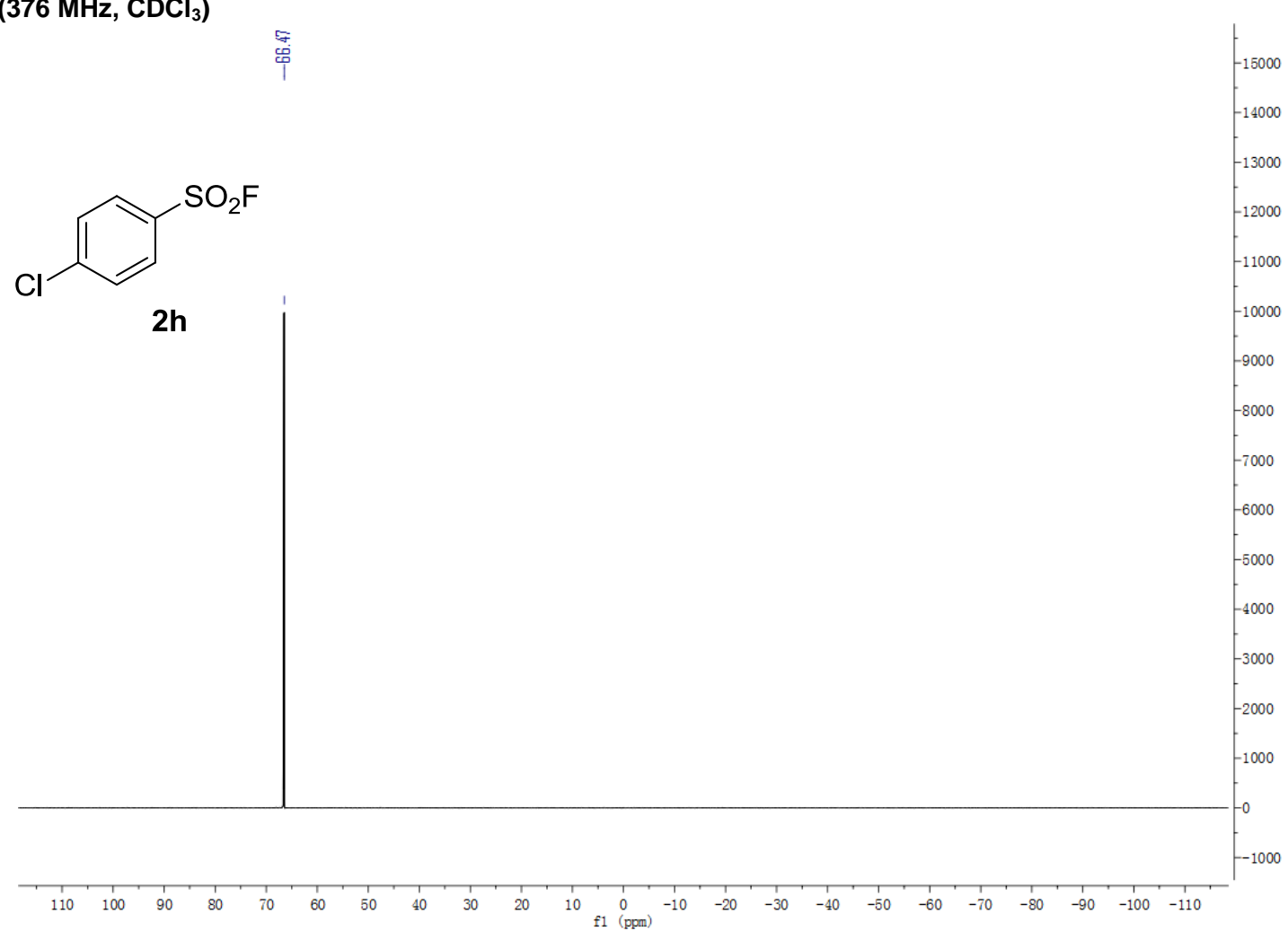


${ }^{1} \mathrm{H}$ NMR $\left(500 \mathrm{MHz}, \mathrm{CDCl}_{3}\right)$

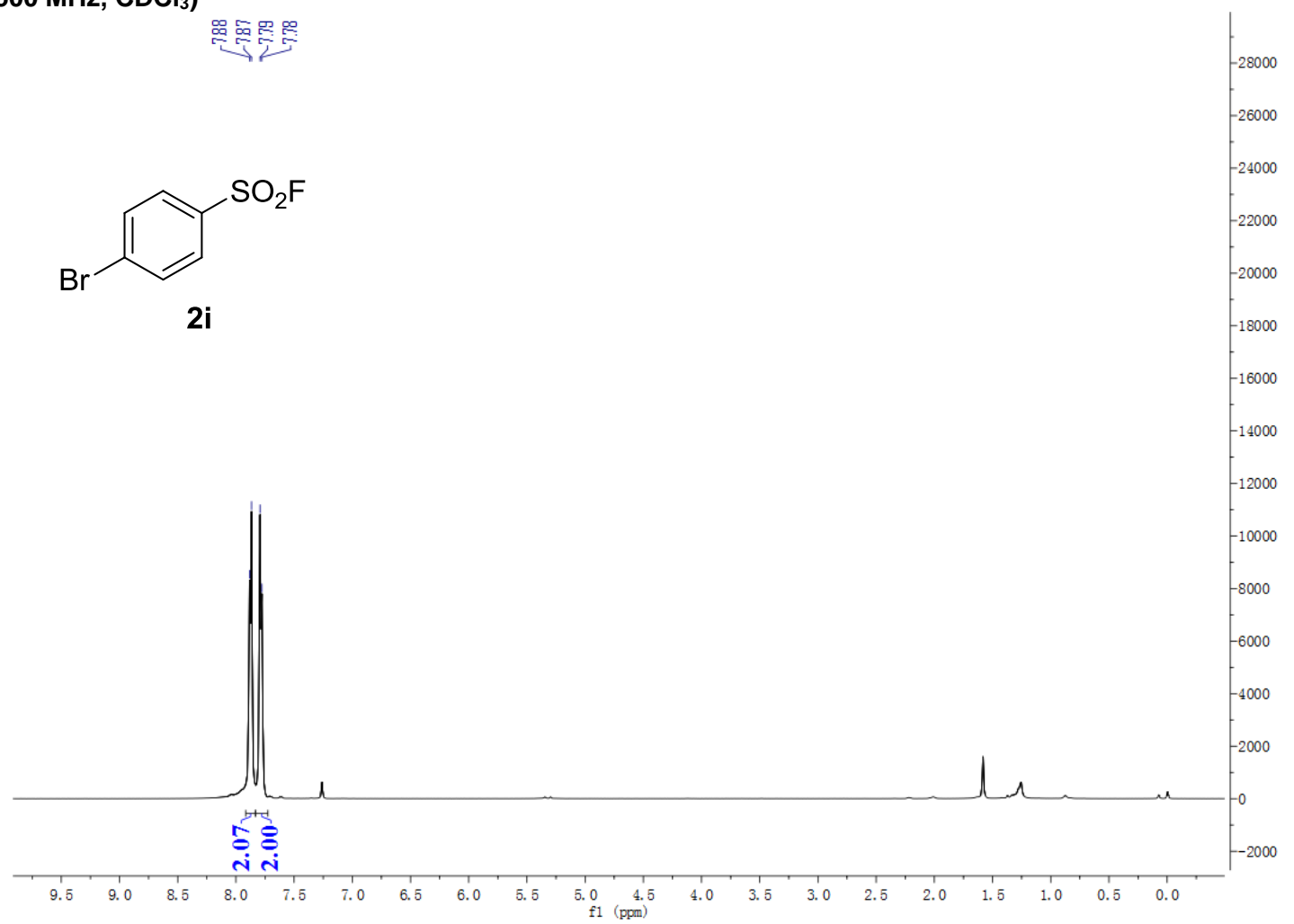

${ }^{13} \mathrm{C} \mathrm{NMR} \mathrm{(101} \mathrm{MHz,} \mathrm{CDCl}_{3}$ )

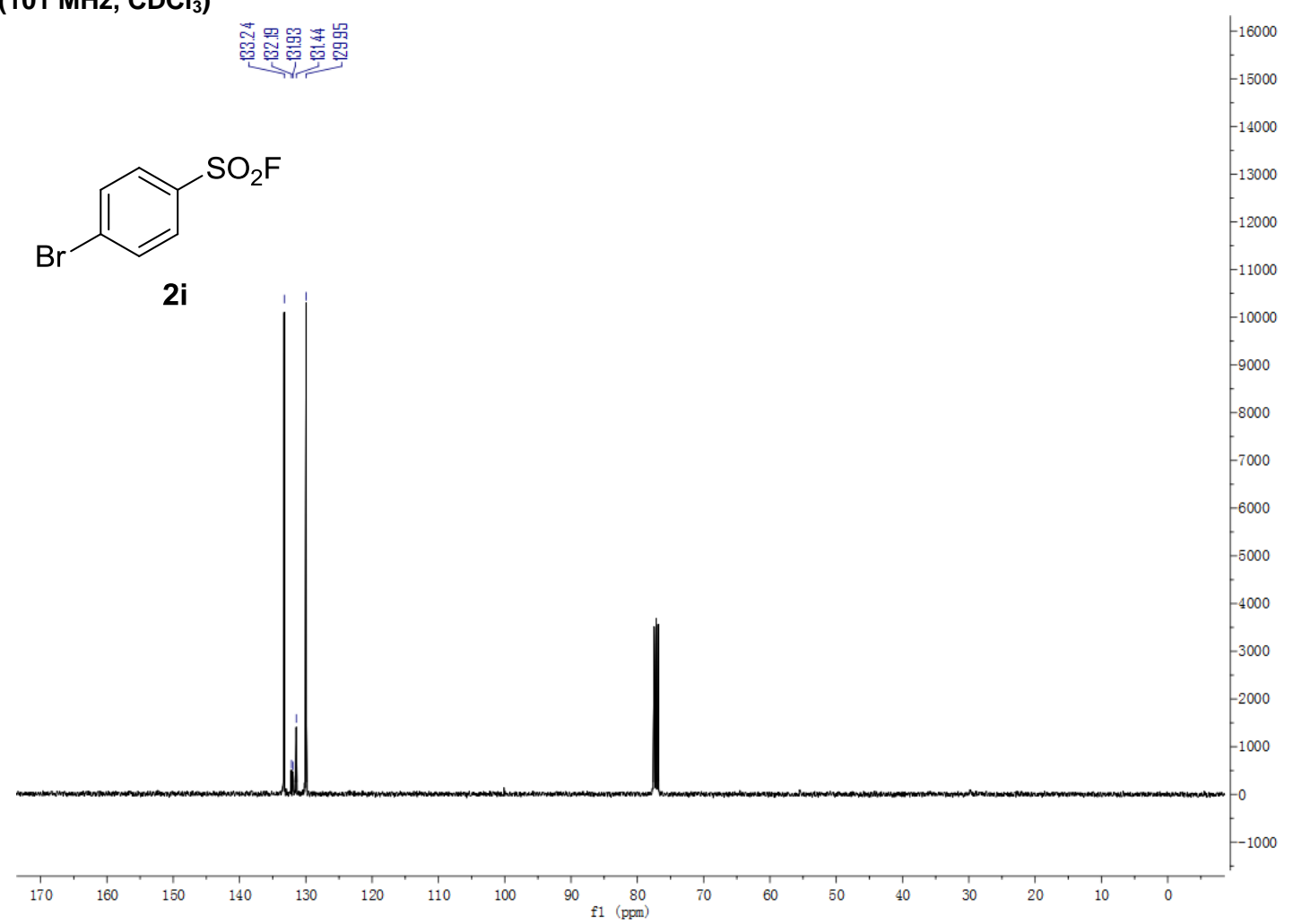


${ }^{19}$ F NMR (376 MHz, $\mathrm{CDCl}_{3}$ )

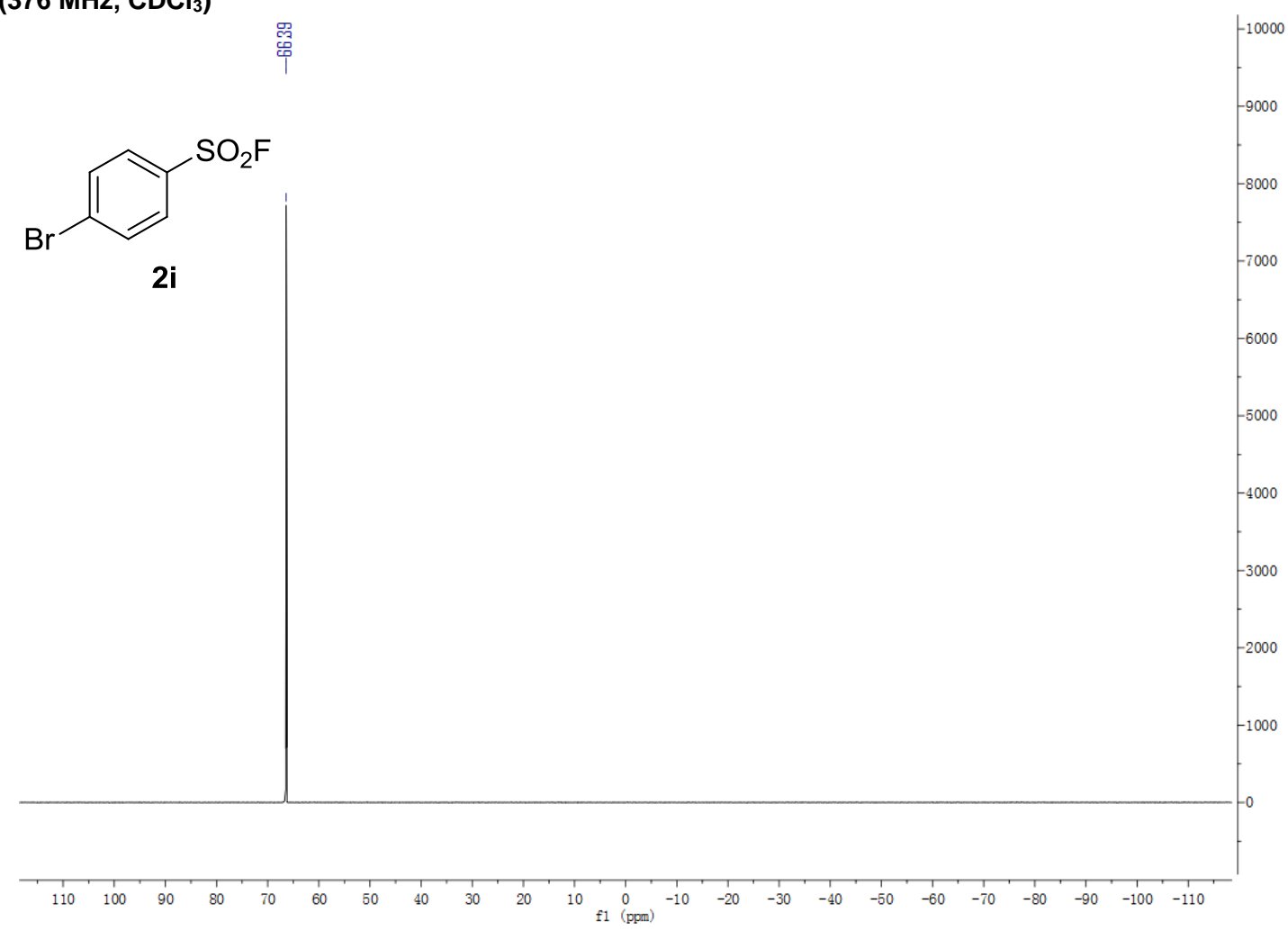

${ }^{1} \mathrm{H}$ NMR (400 MHz, $\mathrm{CDCl}_{3}$ )

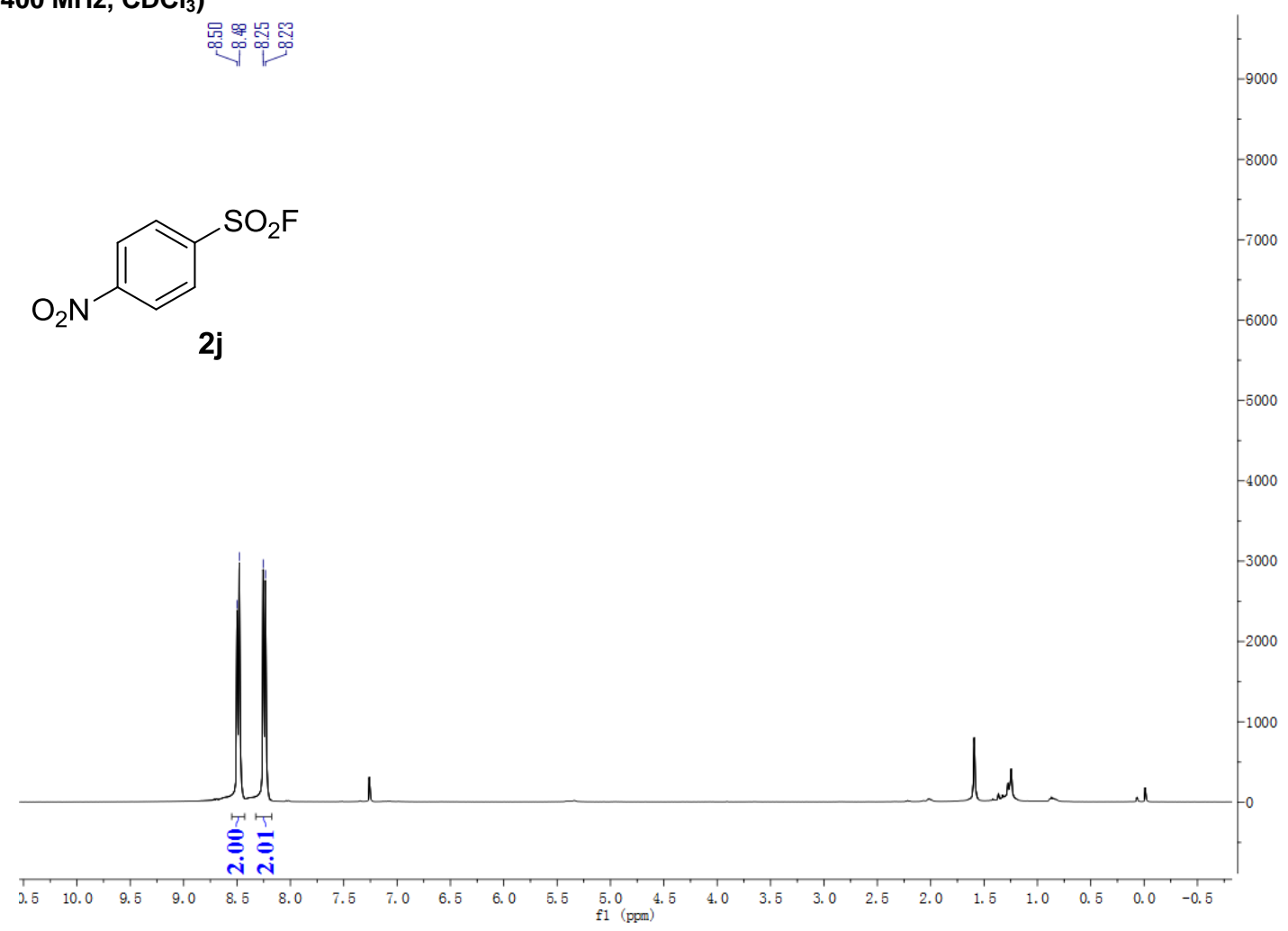


${ }^{13} \mathrm{C}$ NMR (101 MHz, $\mathrm{CDCl}_{3}$ )

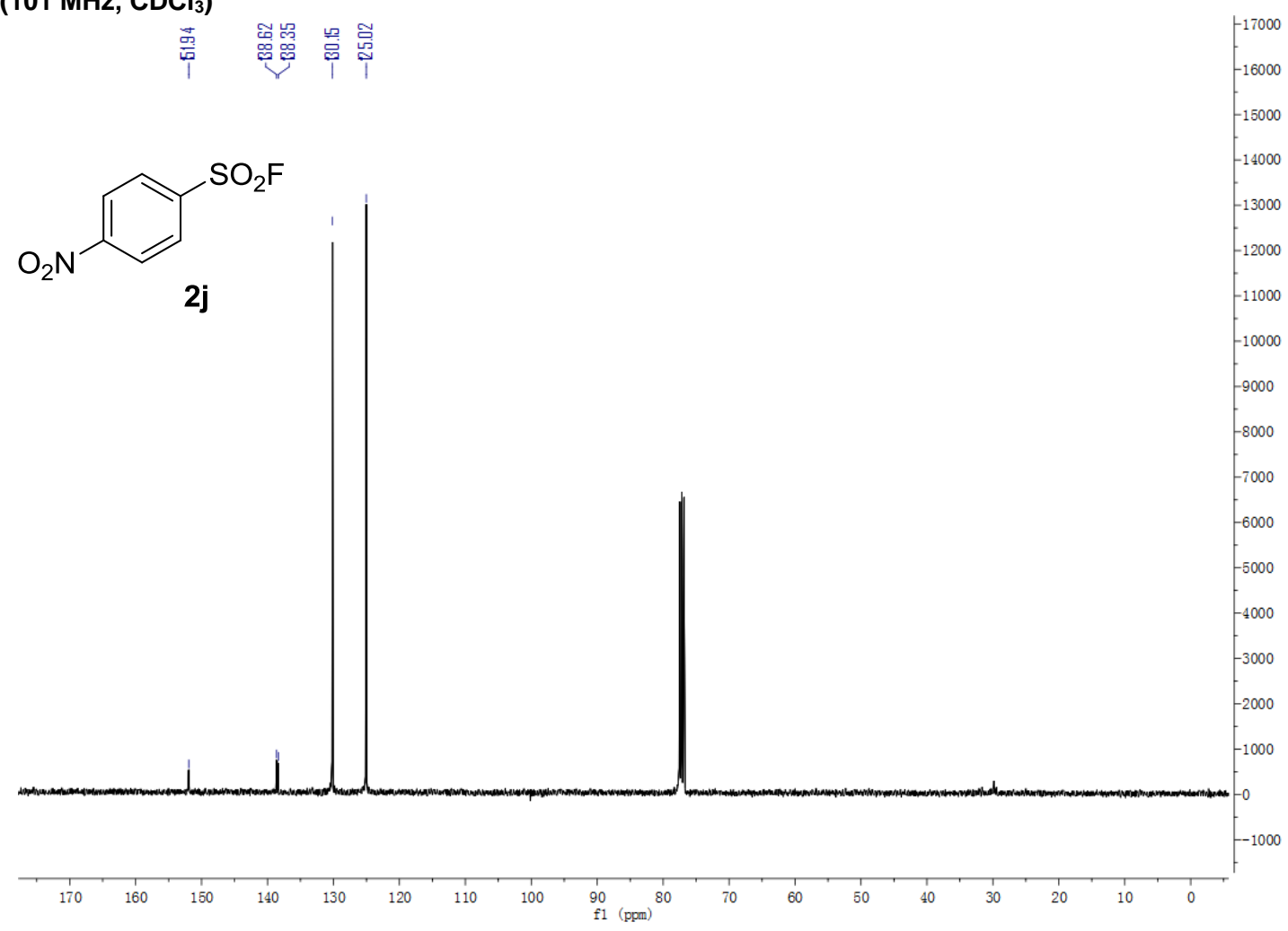

${ }^{19} \mathrm{~F} \mathrm{NMR} \mathrm{(376} \mathrm{MHz,} \mathrm{CDCl}_{3}$ )

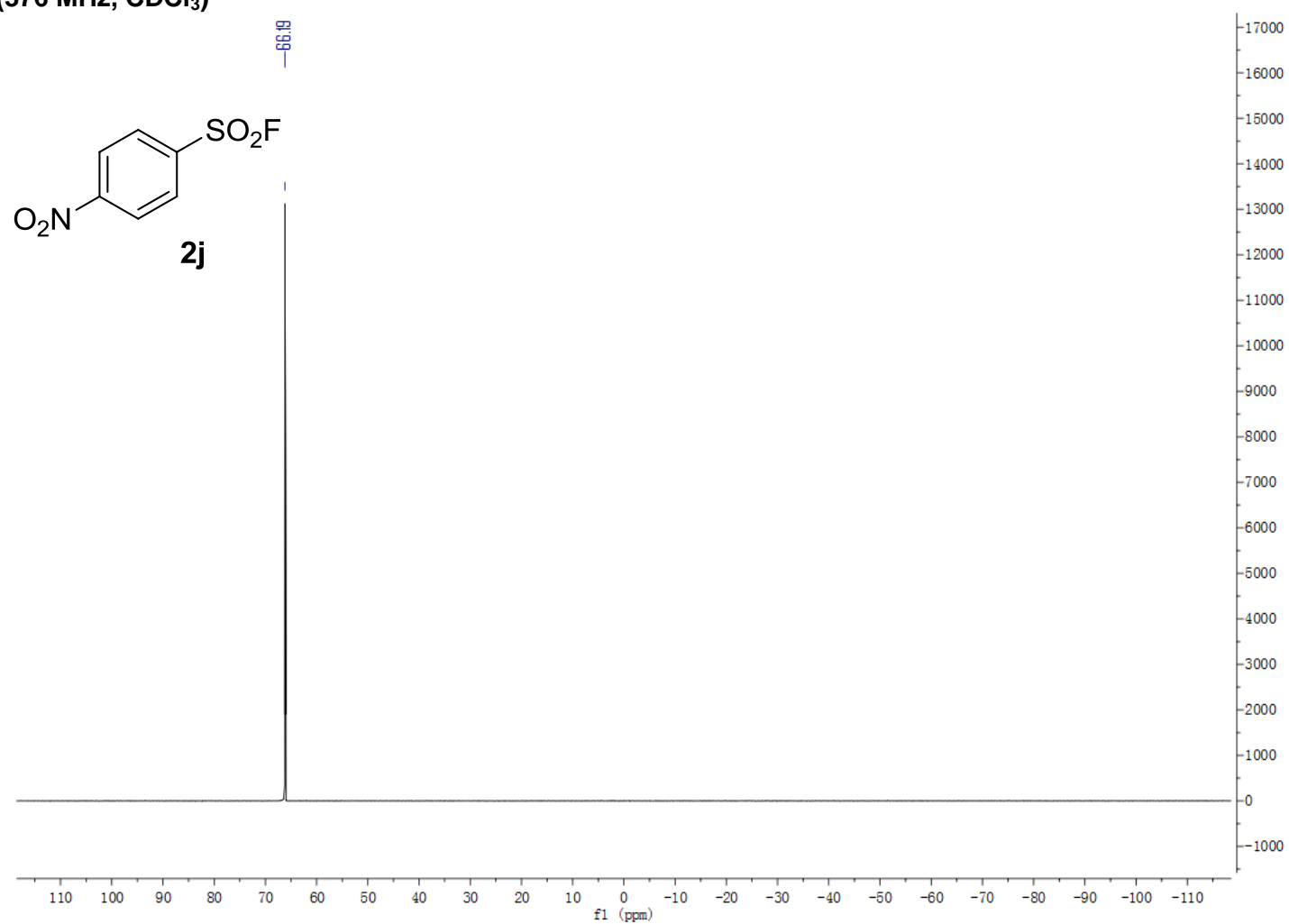


${ }^{1} \mathrm{H}$ NMR (400 MHz, $\left.\mathrm{CDCl}_{3}\right)$

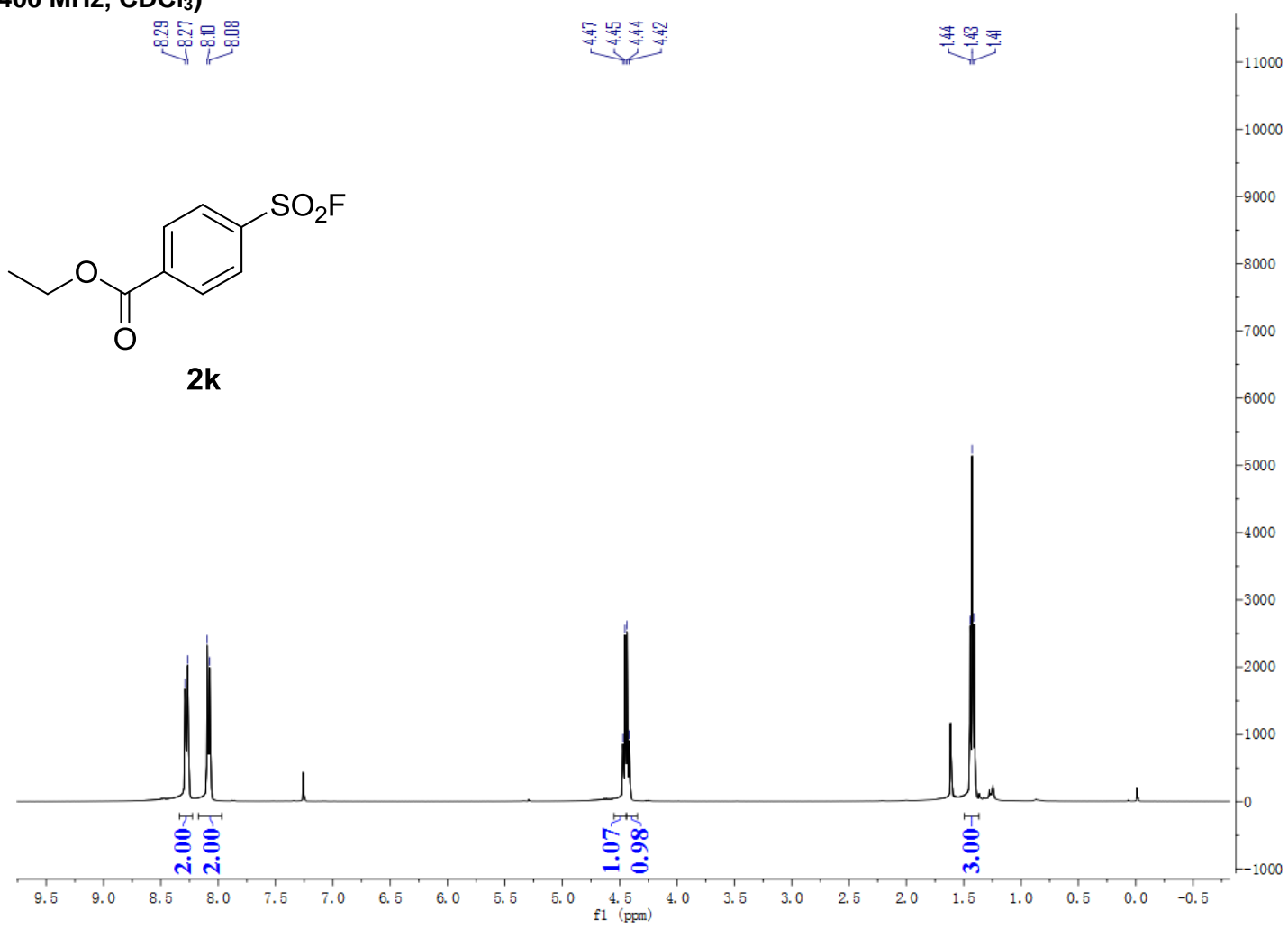

${ }^{13} \mathrm{C}$ NMR (101 MHz, $\left.\mathrm{CDCl}_{3}\right)$

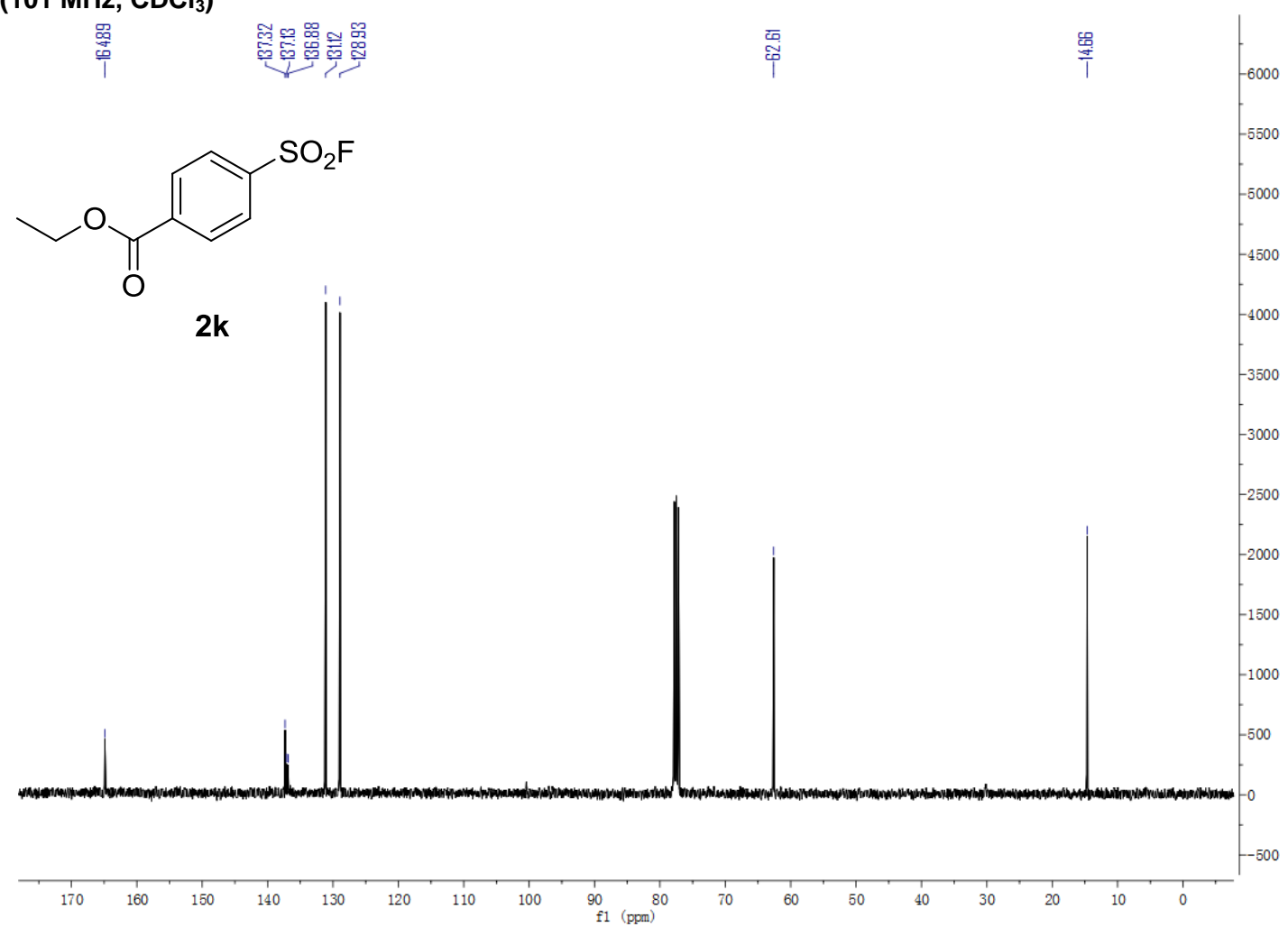


${ }^{19}$ F NMR (376 MHz, $\mathrm{CDCl}_{3}$ )

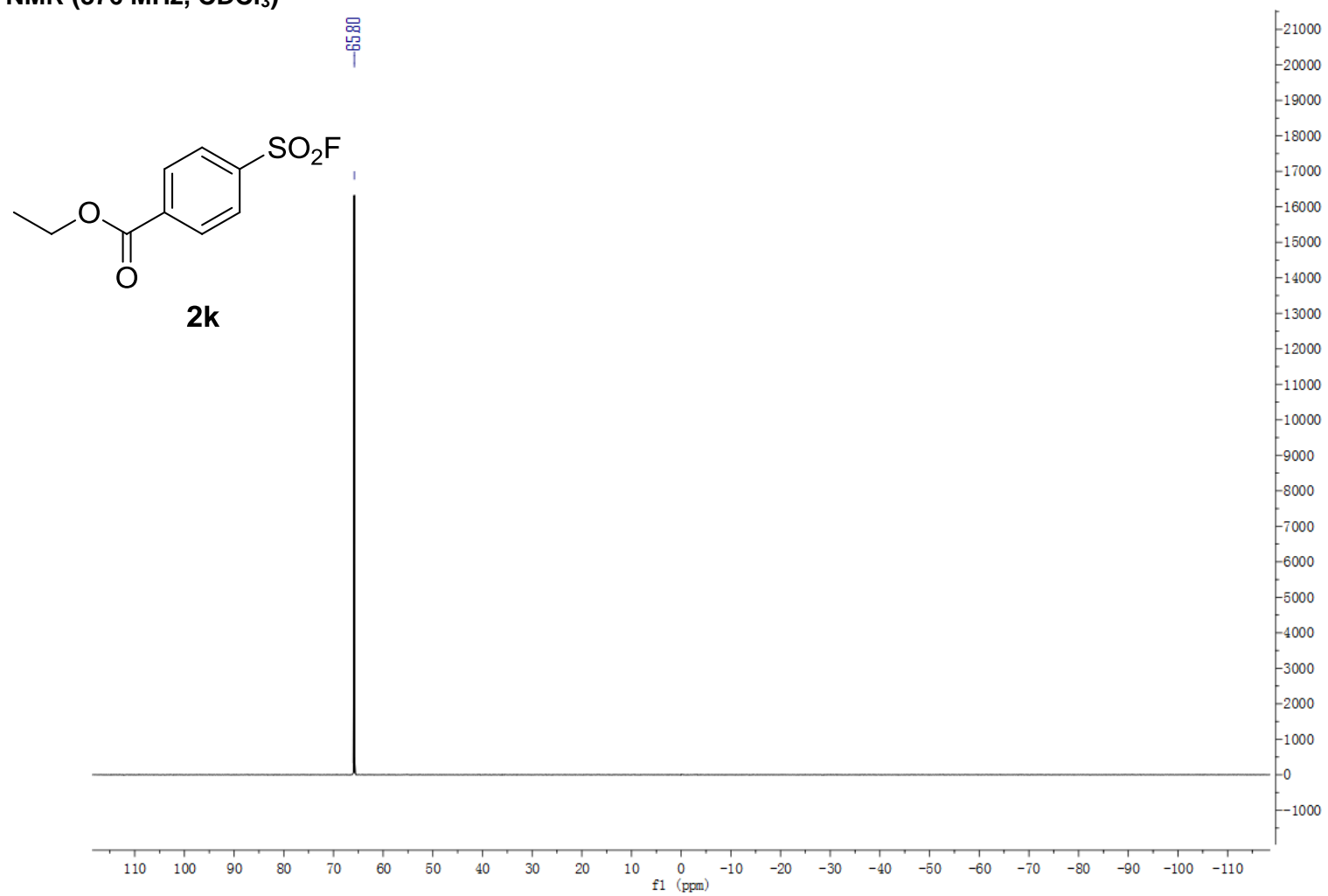

${ }^{1} \mathrm{H}$ NMR (400 MHz, $\mathrm{CDCl}_{3}$ )

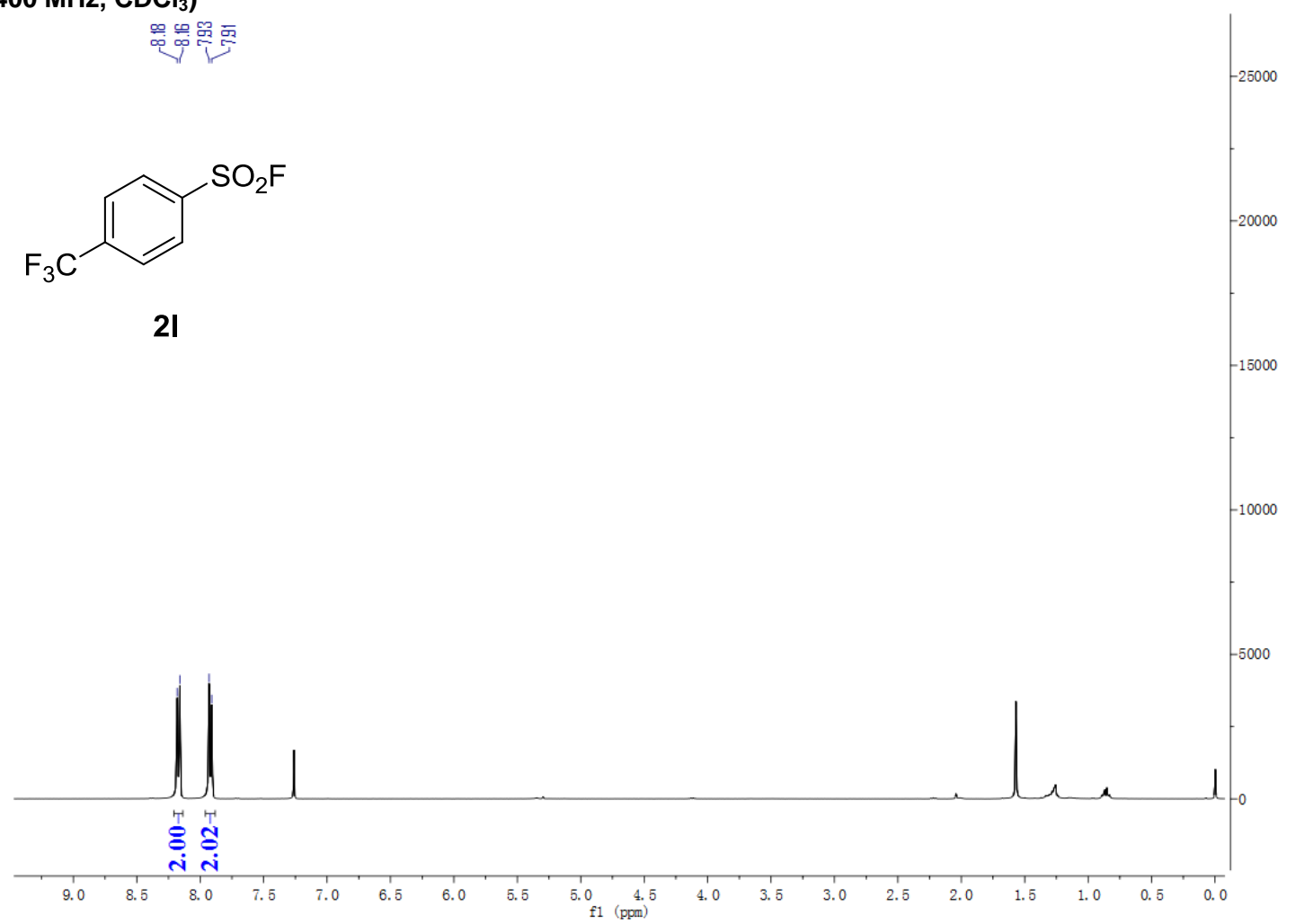


${ }^{13} \mathrm{C} \mathrm{NMR} \mathrm{(101} \mathrm{MHz,} \mathrm{CDCl}_{3}$ )

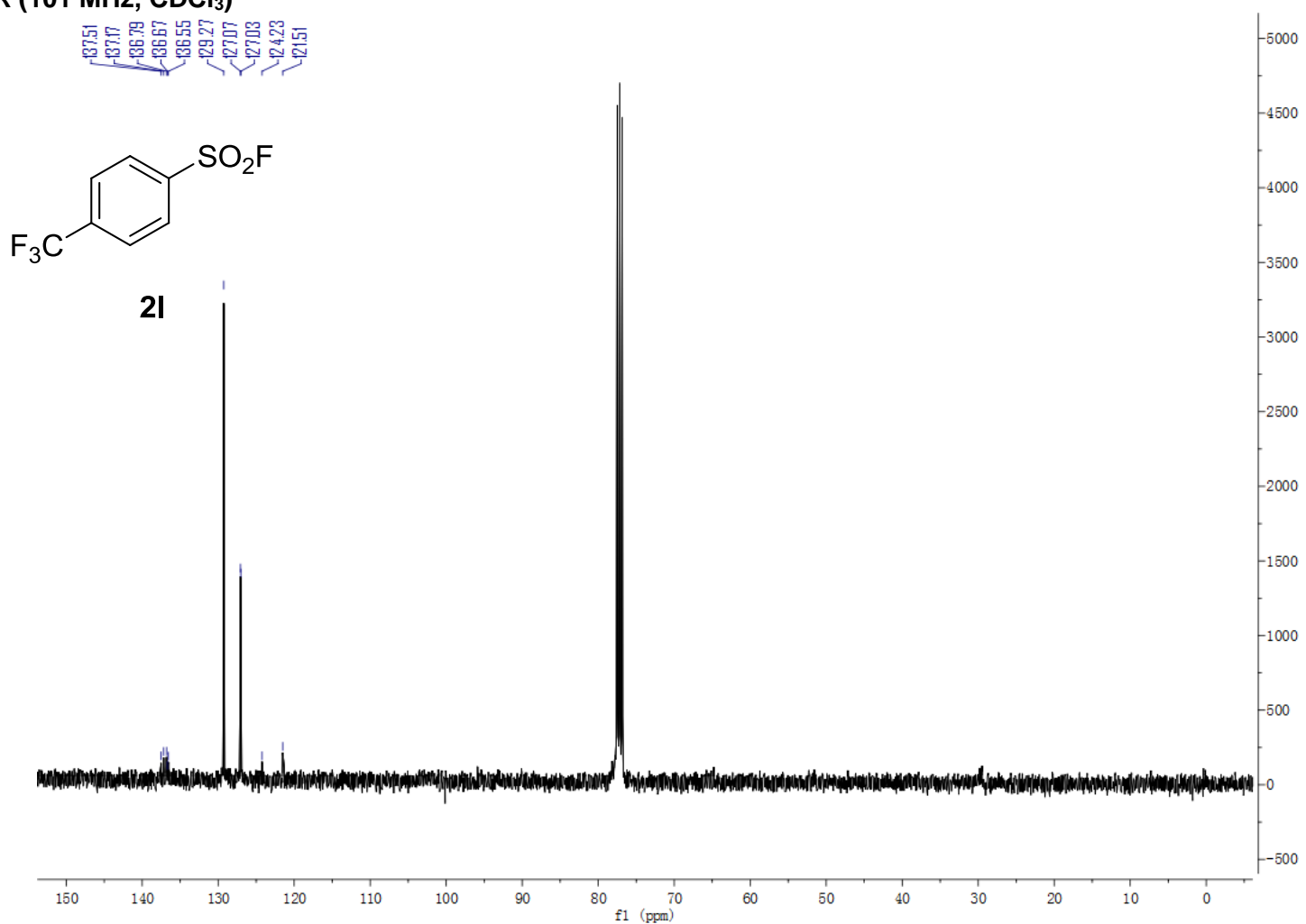

$\left.{ }^{19} \mathrm{~F} \mathrm{NMR} \mathrm{(376} \mathrm{MHz}, \mathrm{CDCl}_{3}\right)$

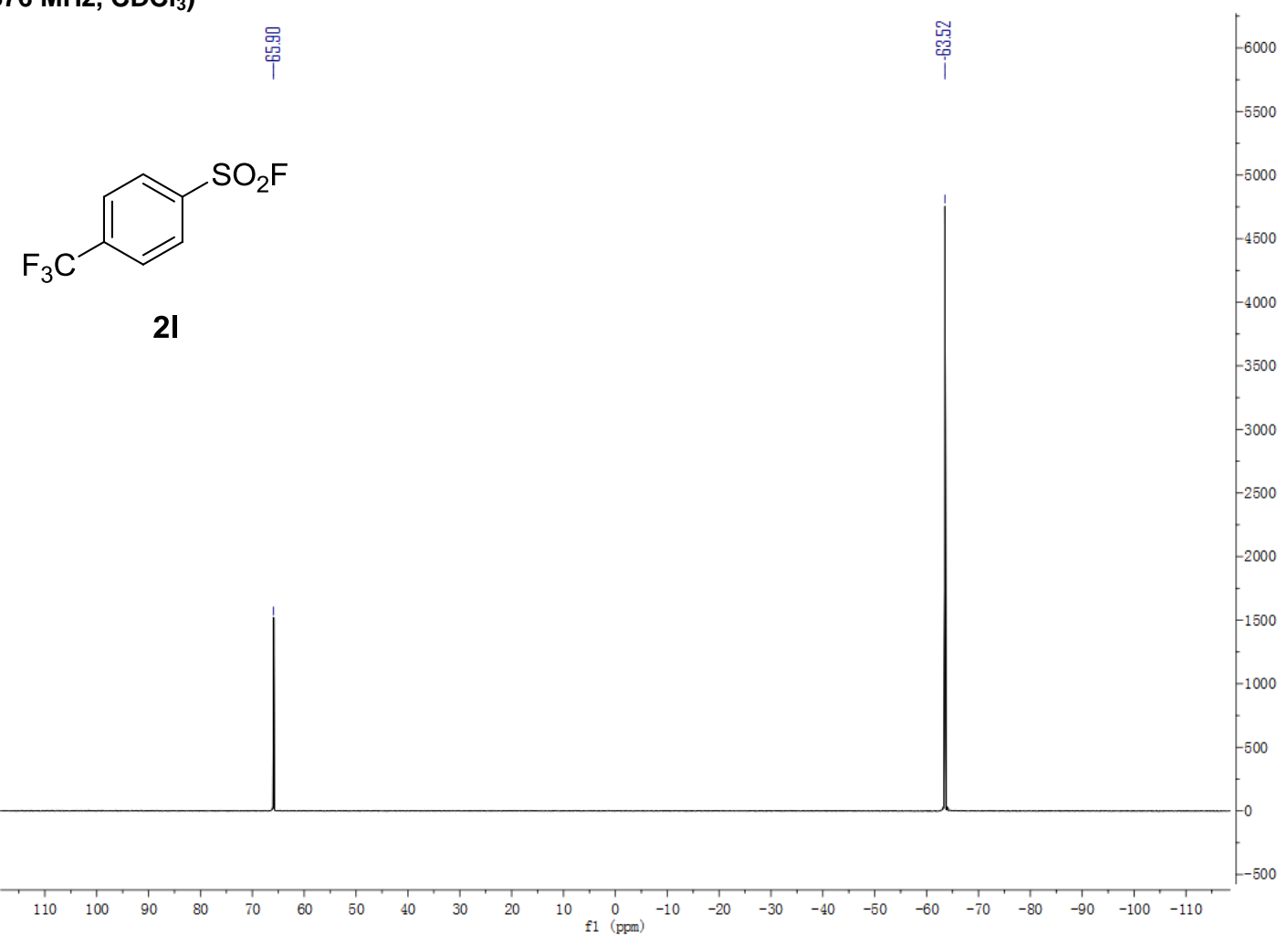


${ }^{1} \mathrm{H}$ NMR $\left(400 \mathrm{MHz}, \mathrm{CDCl}_{3}\right)$

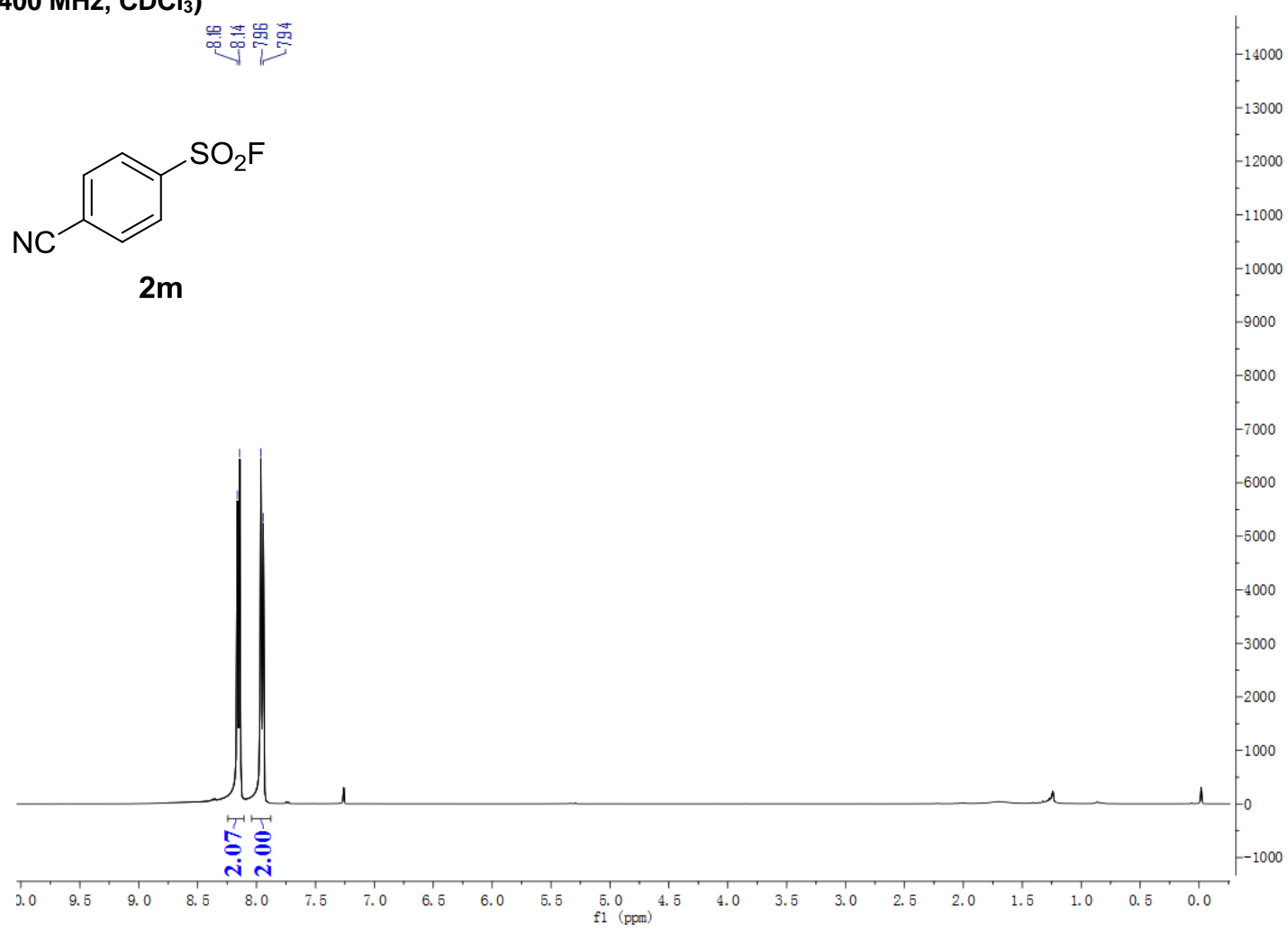

${ }^{13} \mathrm{C}$ NMR (101 MHz, $\mathrm{CDCl}_{3}$ )

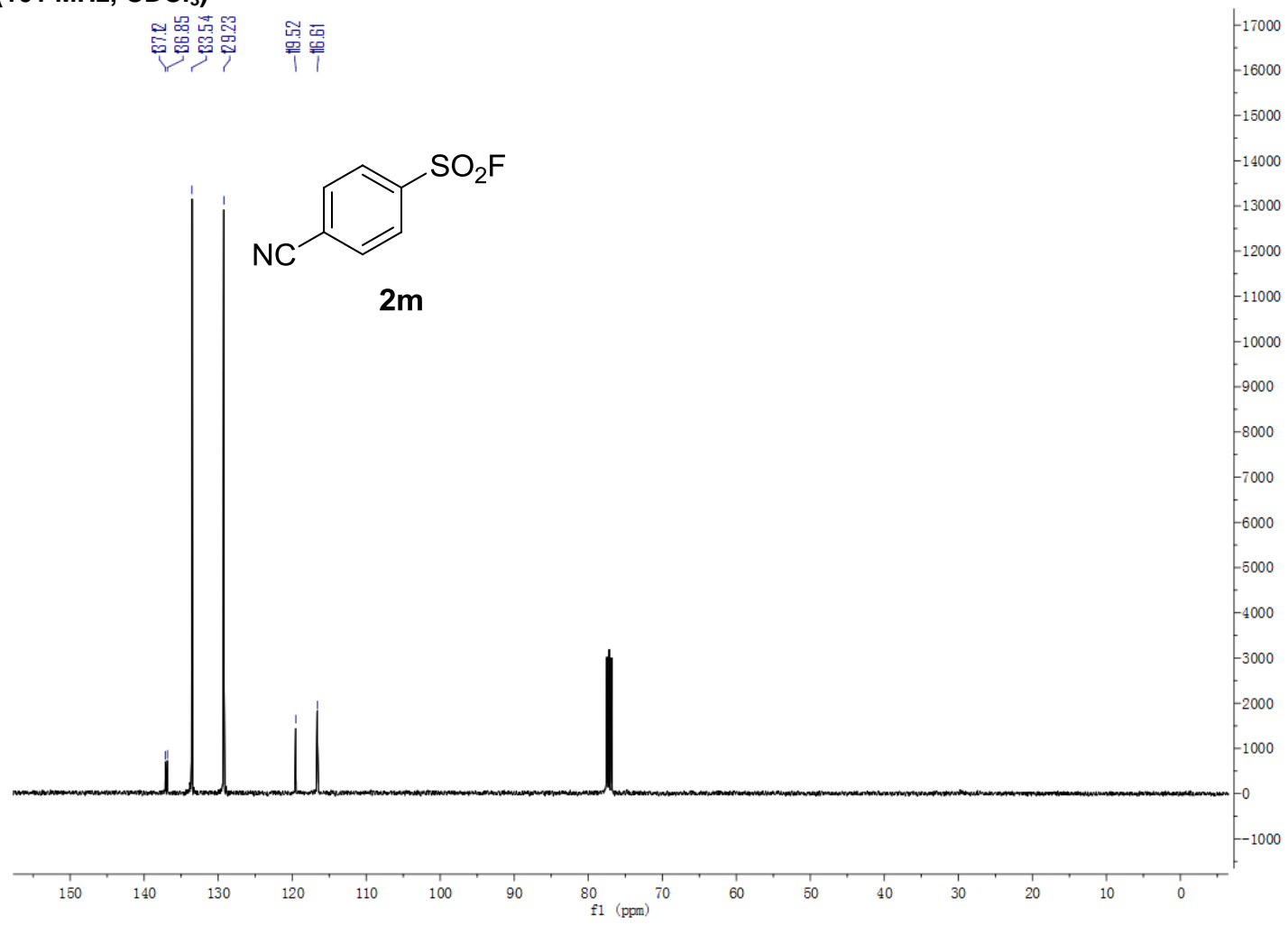


${ }^{19}$ F NMR (376 MHz, $\mathrm{CDCl}_{3}$ )

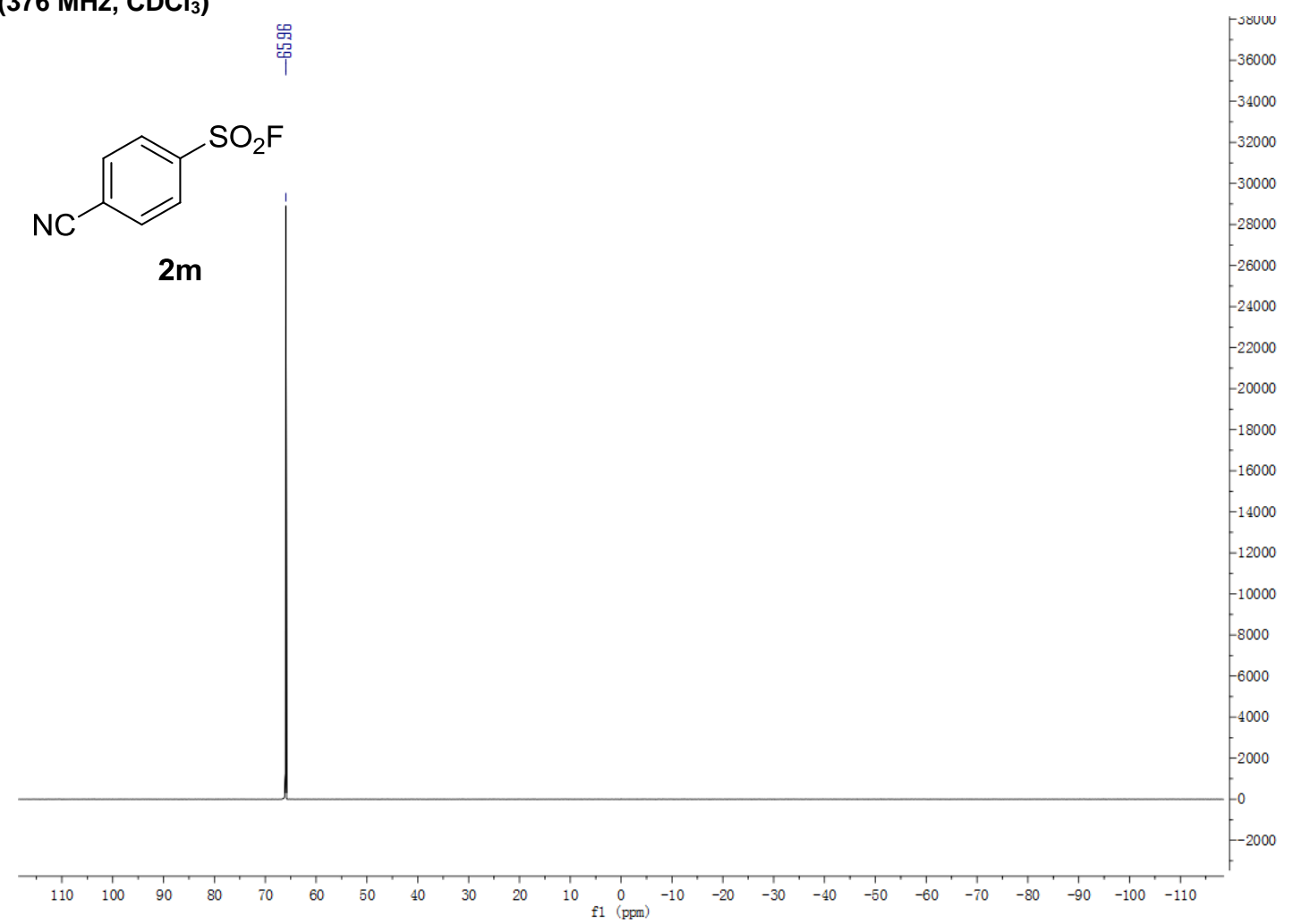

${ }^{1} \mathrm{H}$ NMR (500 MHz, $\mathrm{CDCl}_{3}$ )

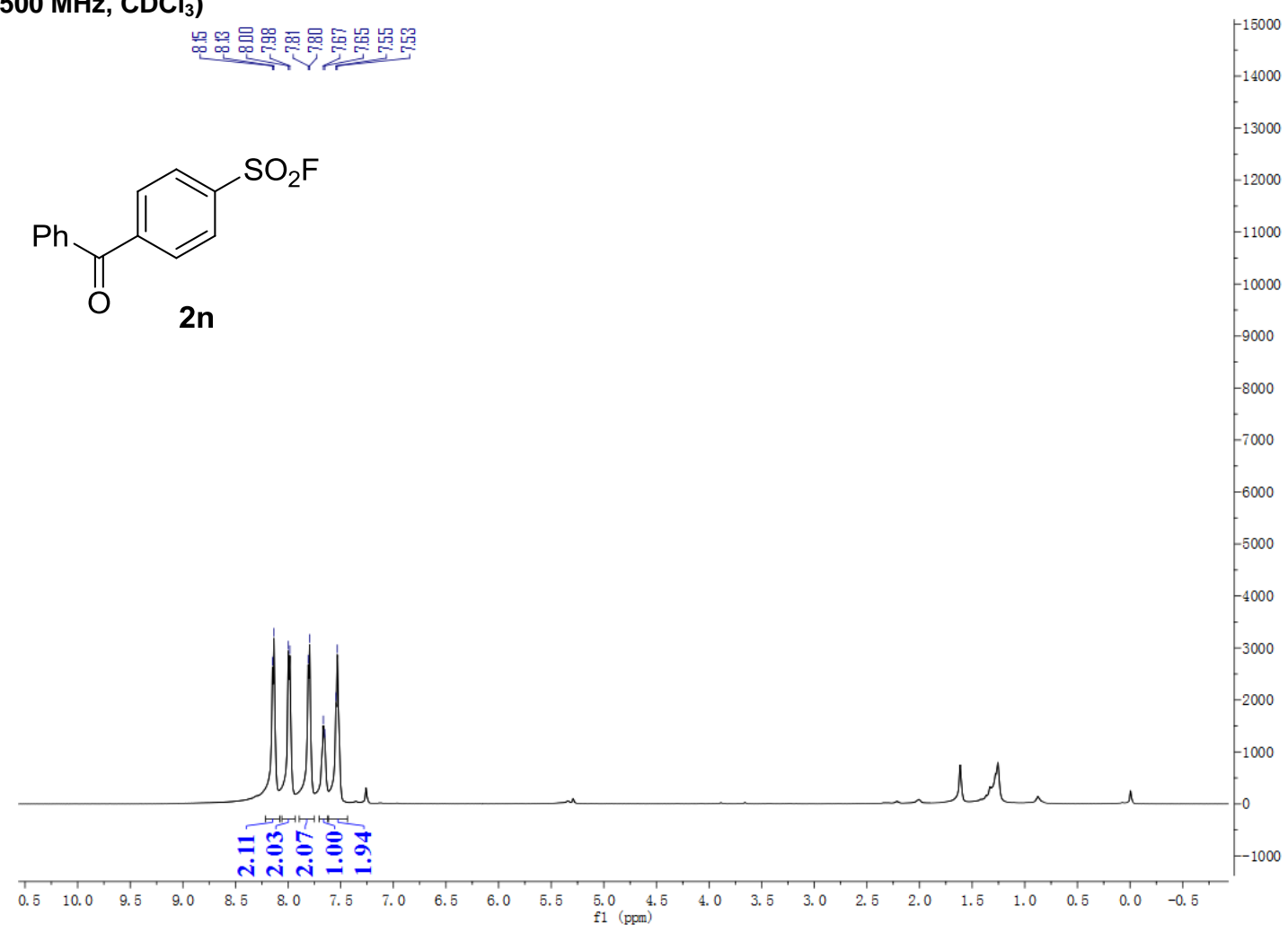


${ }^{13} \mathrm{C}$ NMR (101 $\mathrm{MHz}, \mathrm{CDCl}_{3}$ )

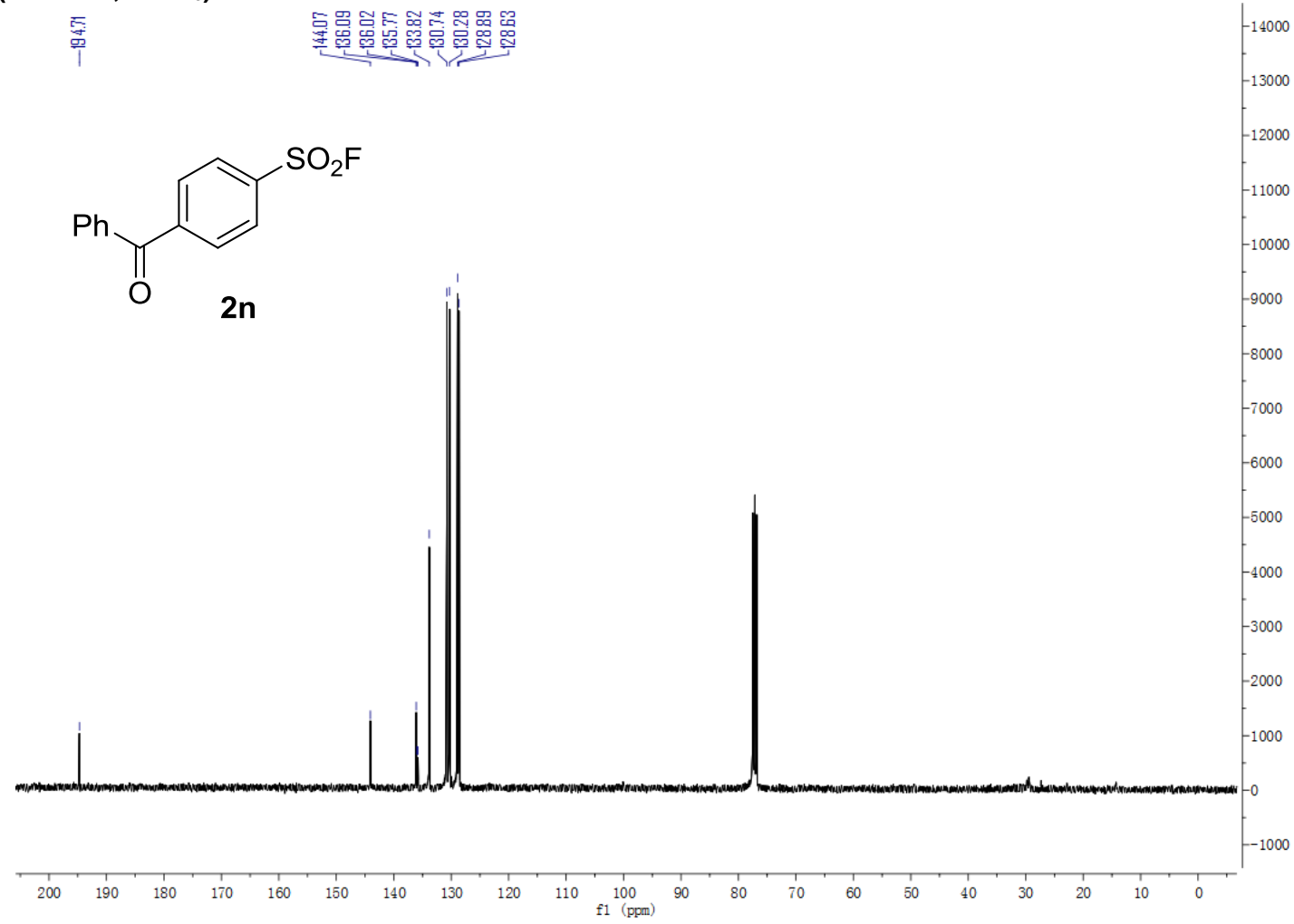

${ }^{19} \mathrm{~F} \mathrm{NMR} \mathrm{(471} \mathrm{MHz,} \mathrm{CDCl}_{3}$ )

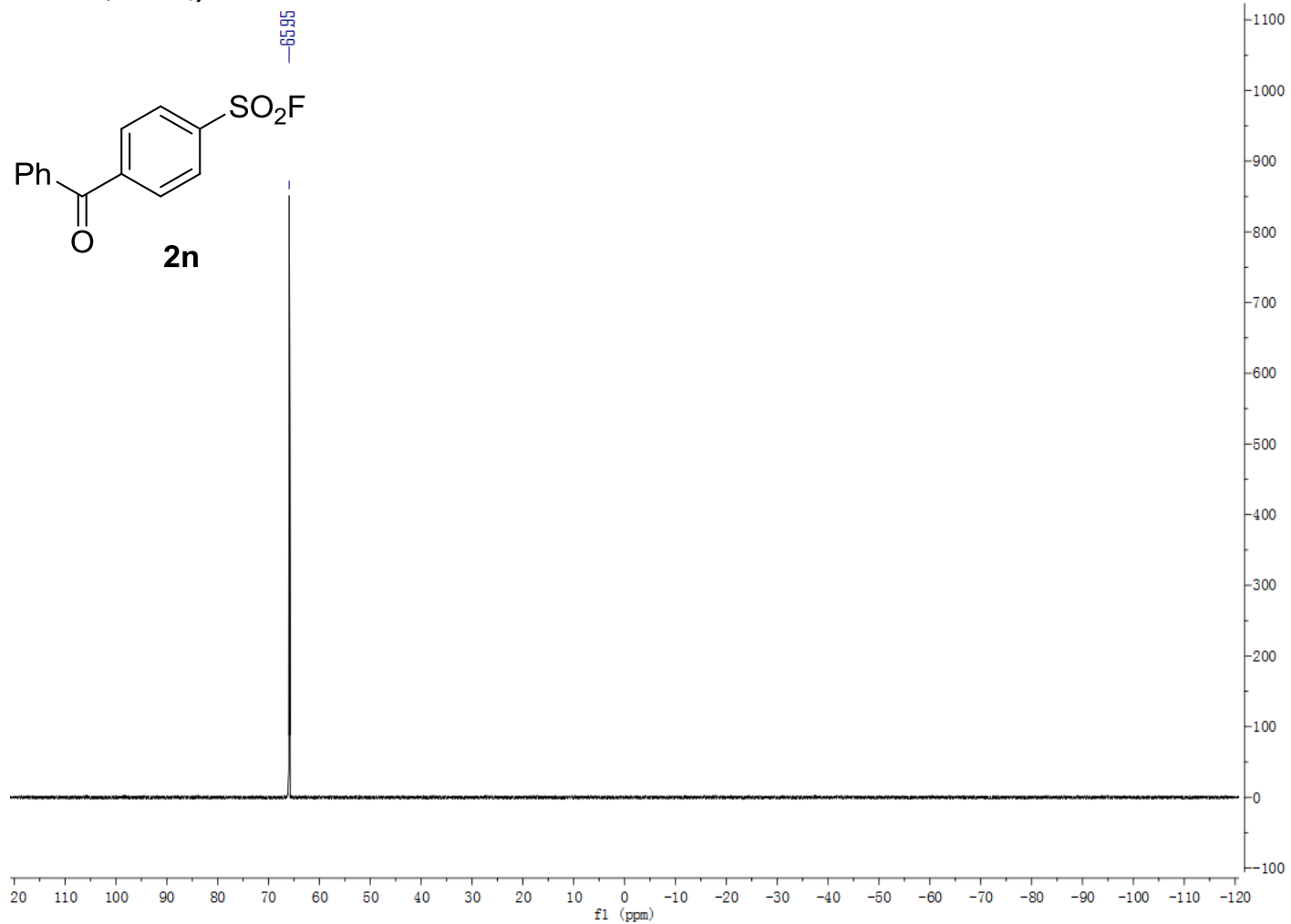


${ }^{1} \mathrm{H}$ NMR (400 MHz, DMSO-d )

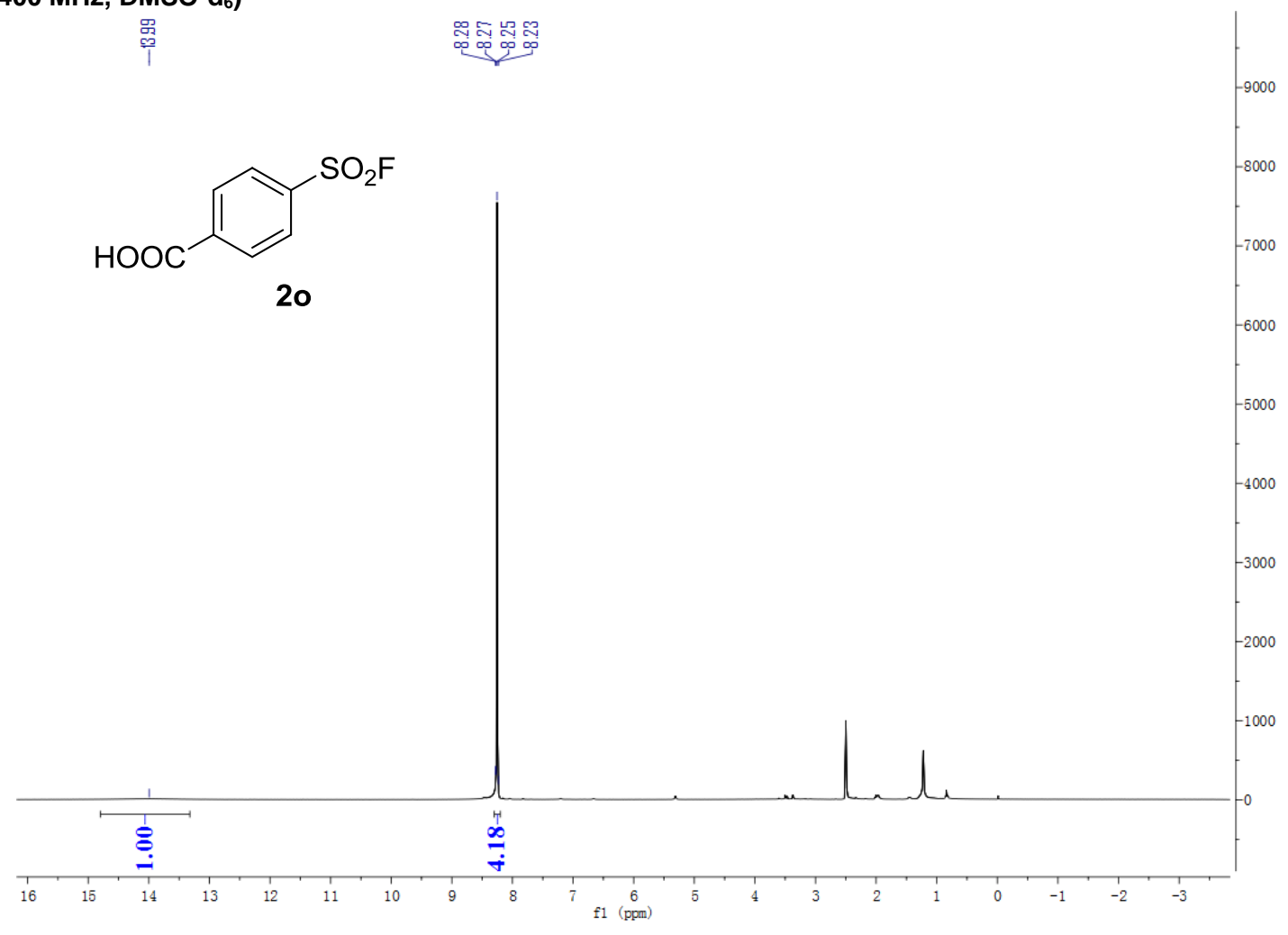

${ }^{13} \mathrm{C}$ NMR (101 MHz, DMSO-d 6 )

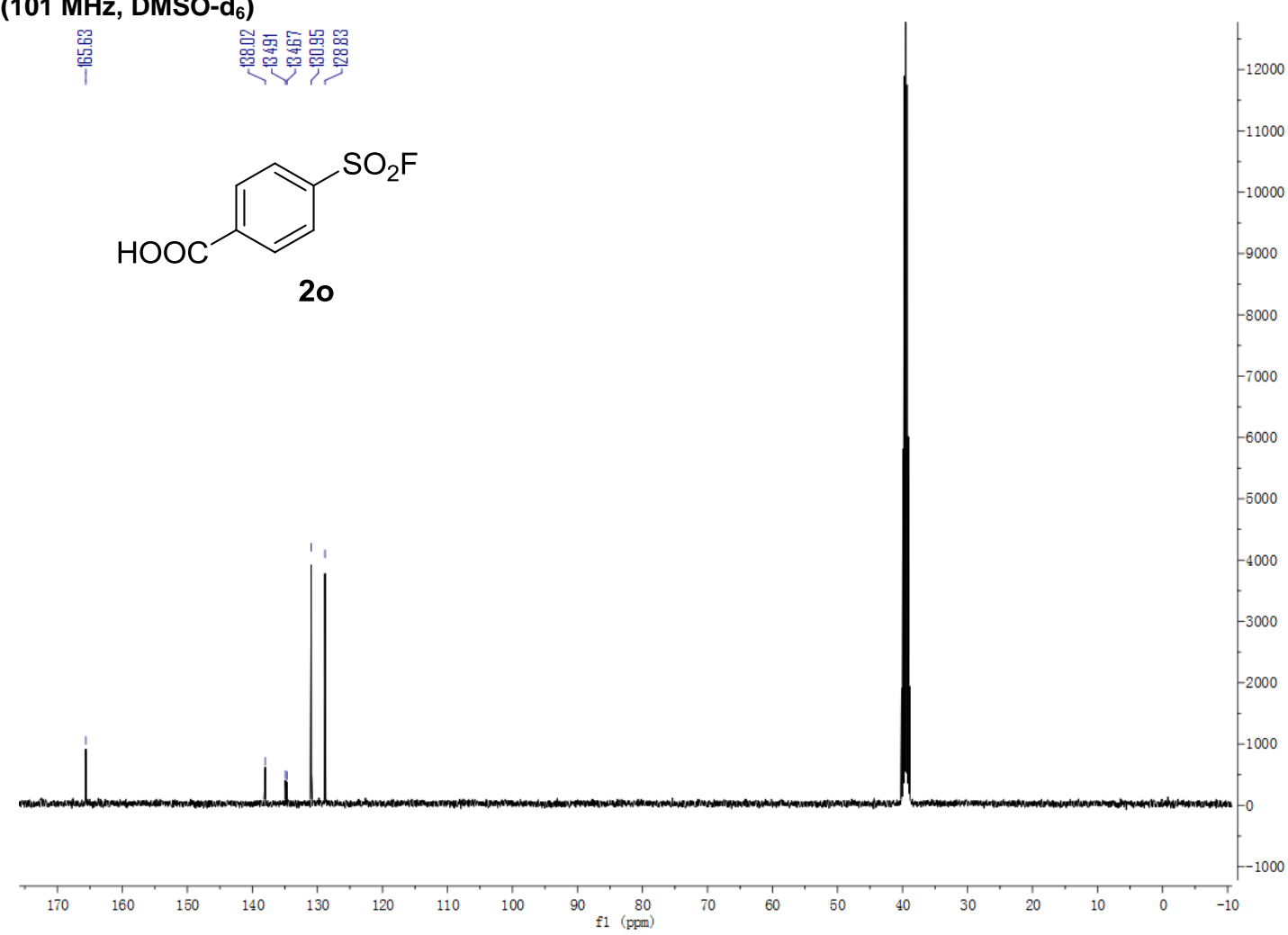


${ }^{19}$ F NMR (376 MHz, DMSO-d 6 )

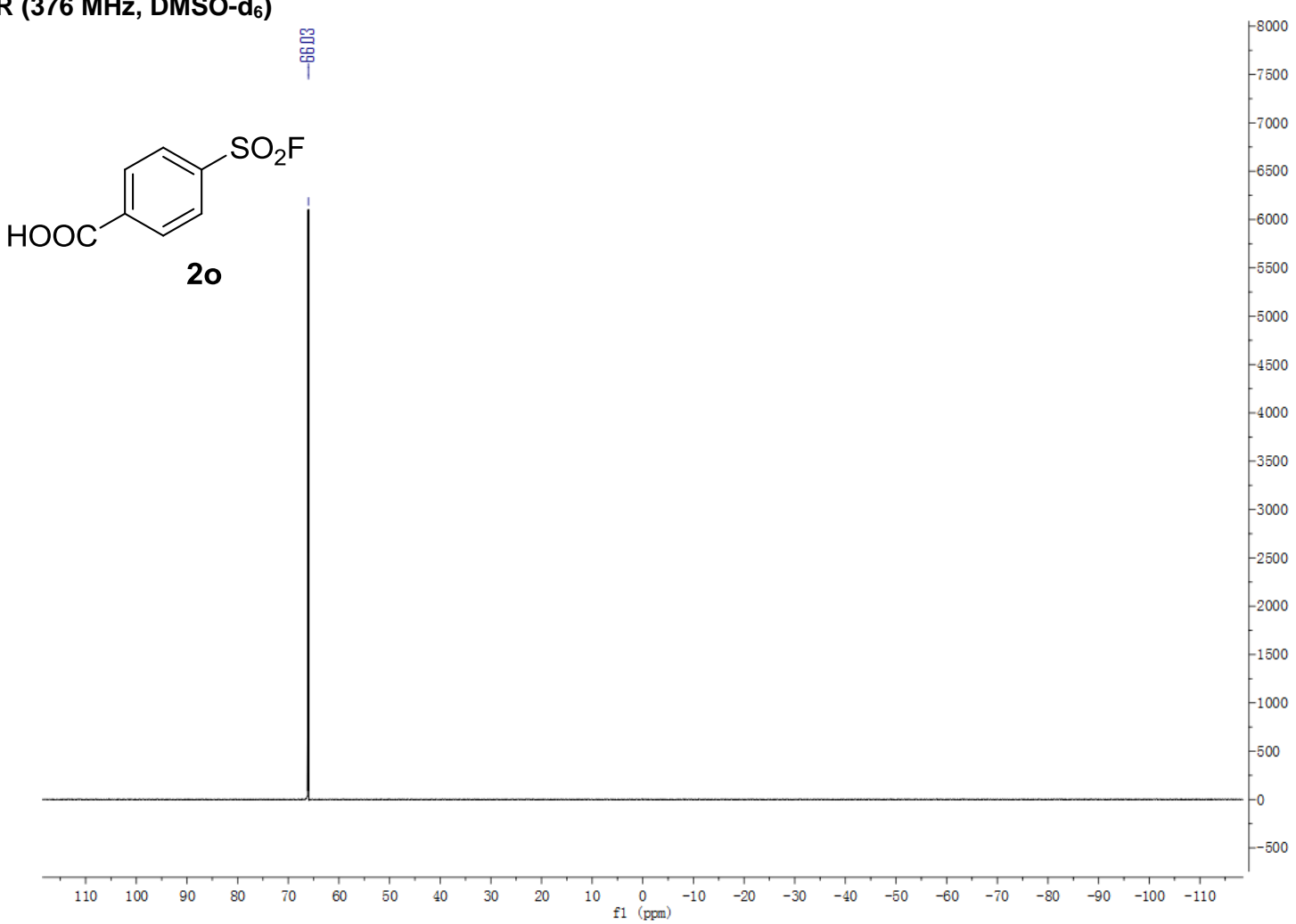

${ }^{1} \mathrm{H}$ NMR (400 MHz, $\mathrm{CDCl}_{3}$ )

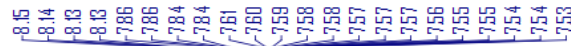

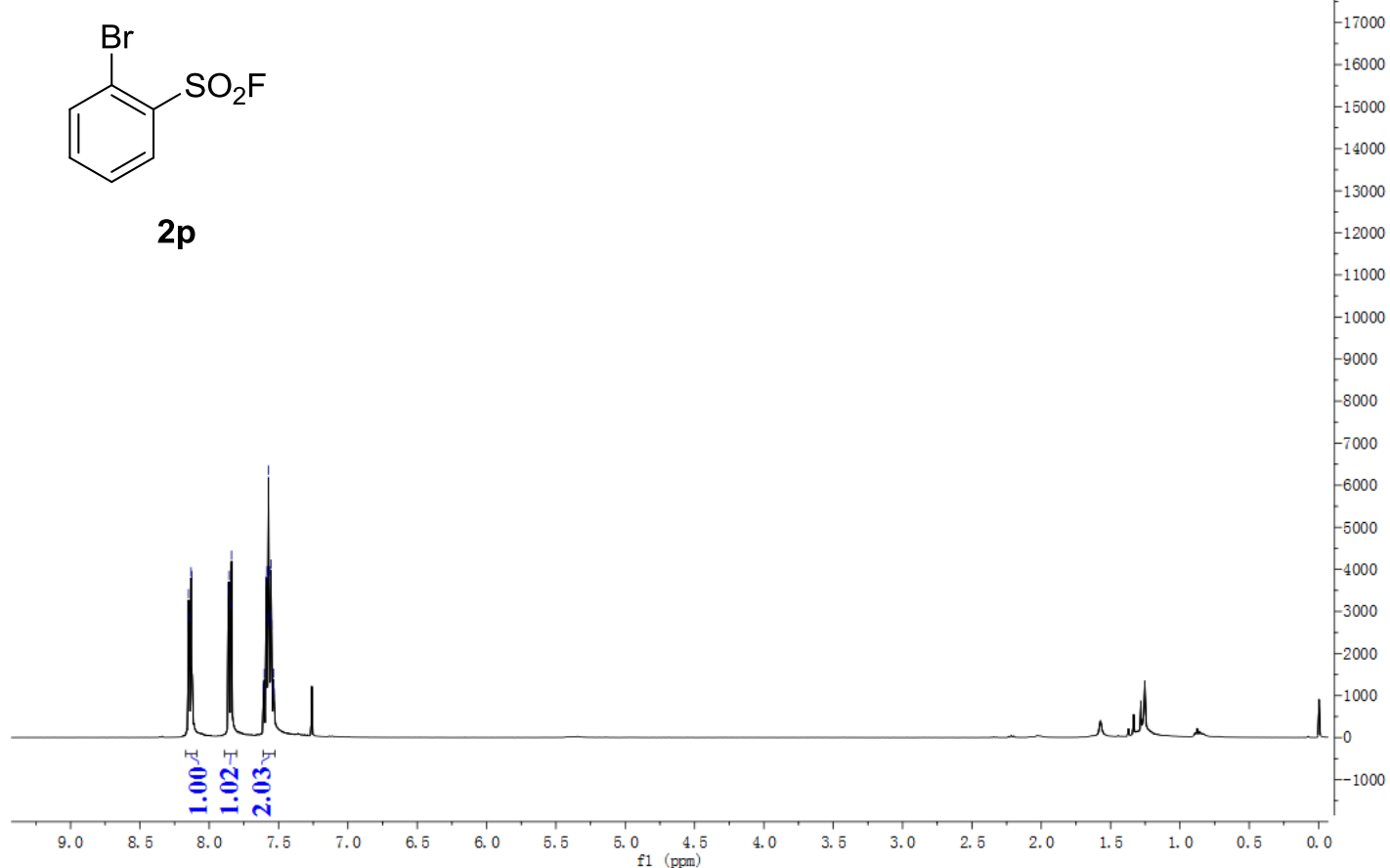


${ }^{13} \mathrm{C}$ NMR (101 MHz, $\mathrm{CDCl}_{3}$ )

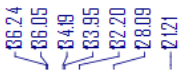<smiles>O=S(=O)(OF)c1ccccc1Br</smiles>

$2 p$

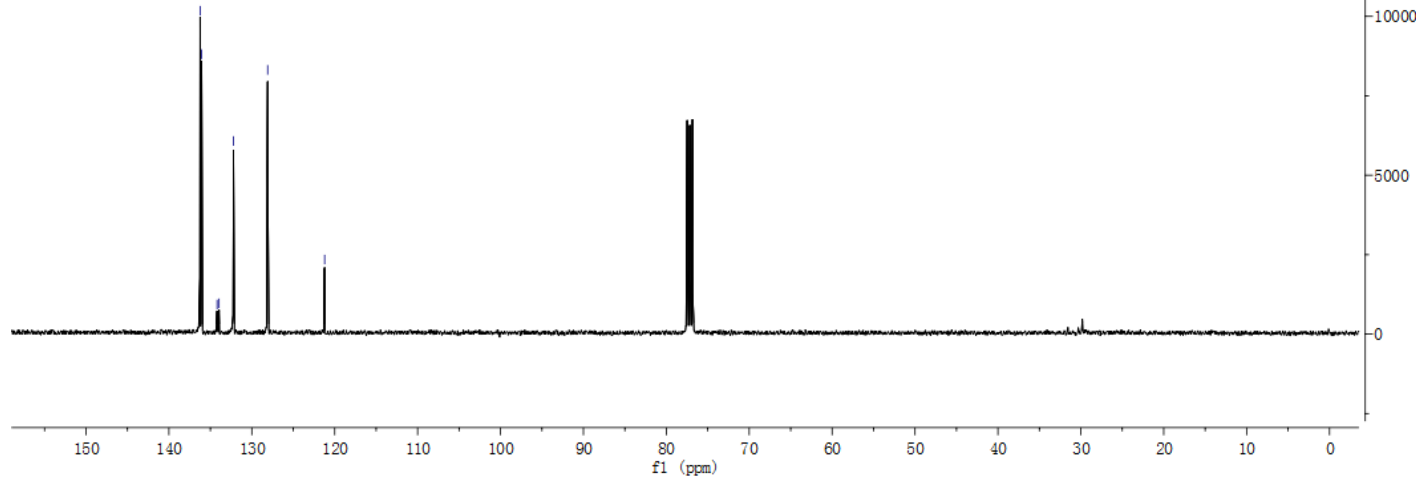

${ }^{19} \mathrm{~F} \mathrm{NMR}\left(376 \mathrm{MHz}, \mathrm{CDCl}_{3}\right.$ )

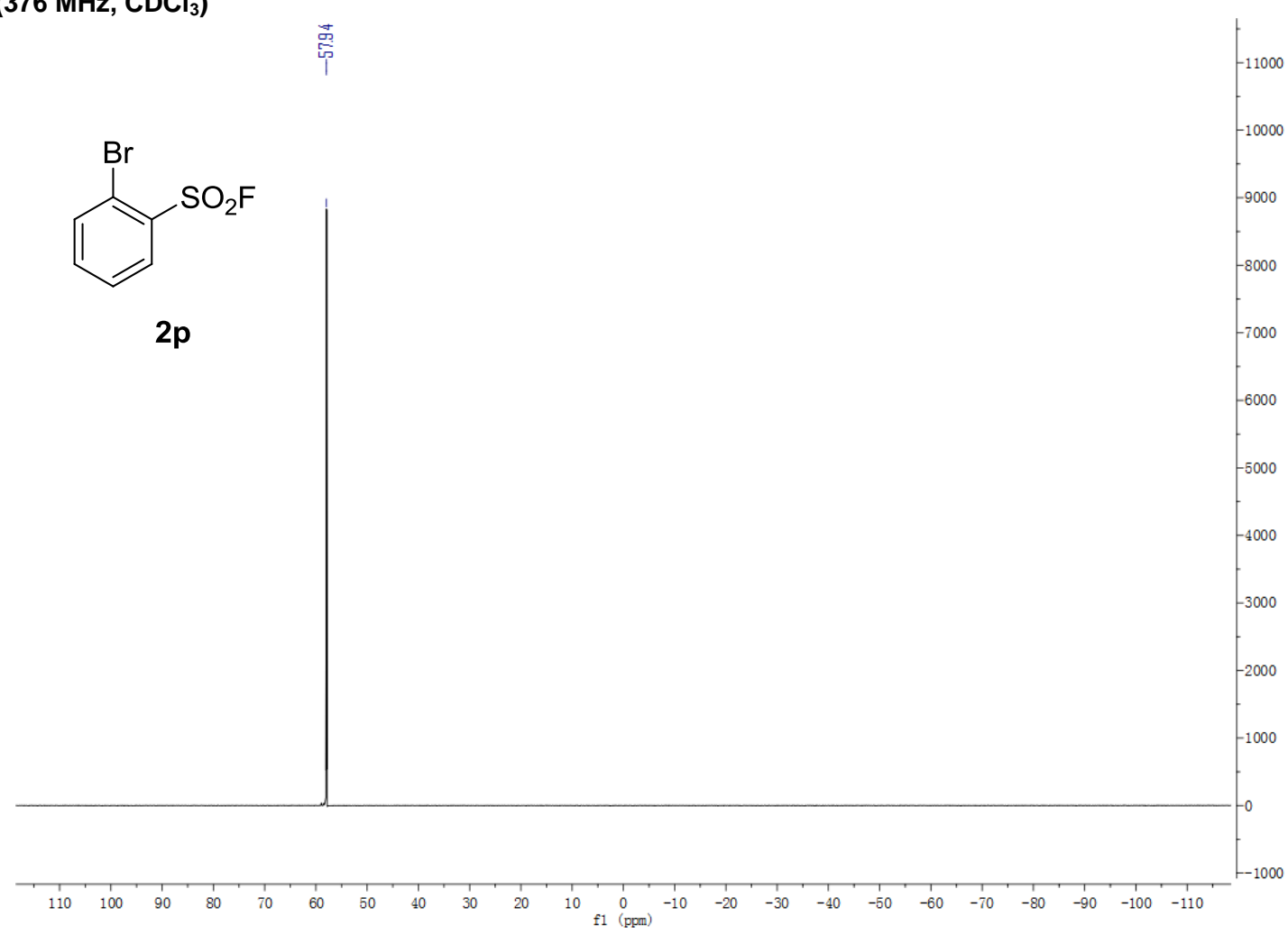


${ }^{1} \mathrm{H}$ NMR $\left(400 \mathrm{MHz}, \mathrm{CDCl}_{3}\right)$

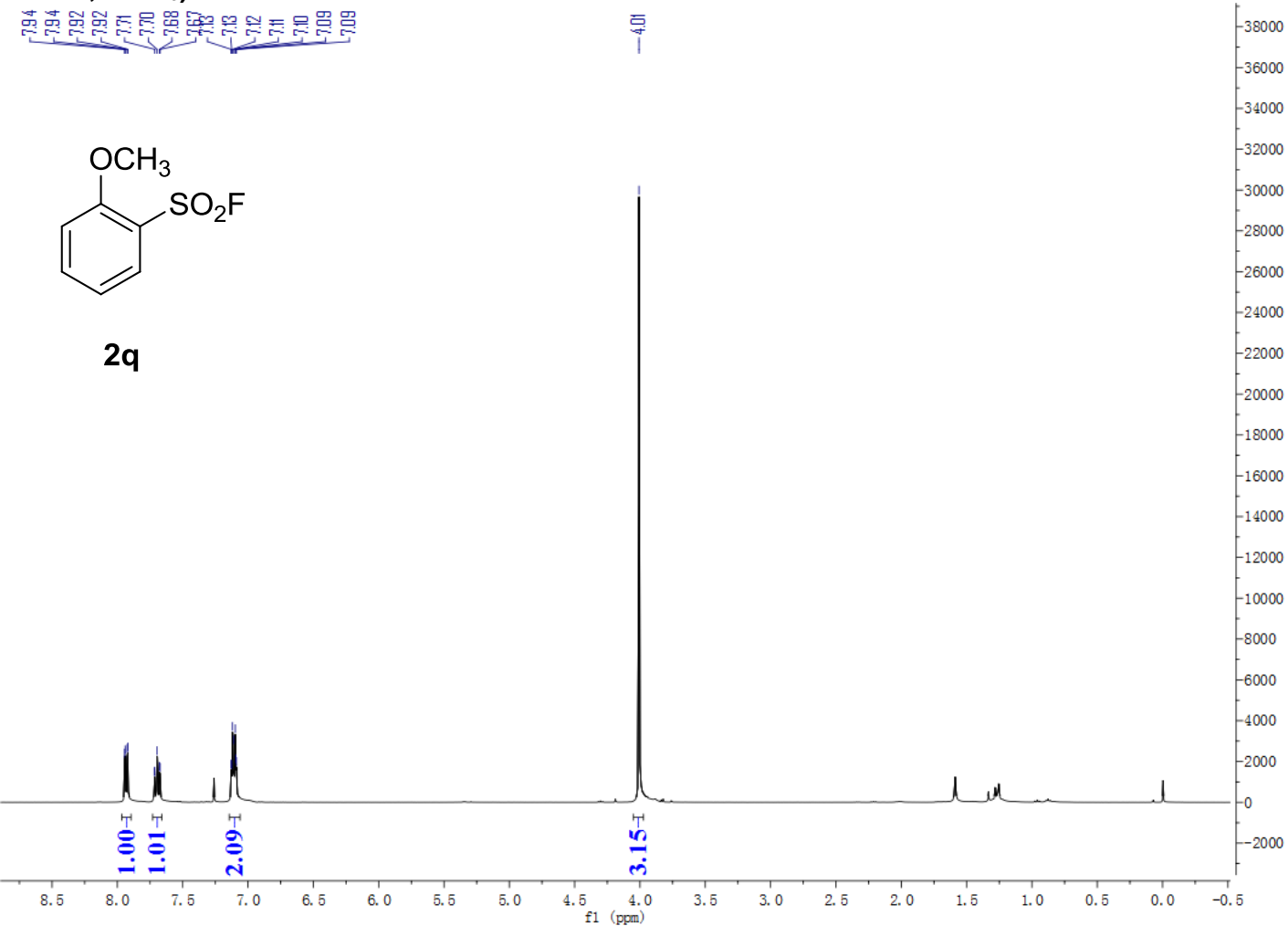

${ }^{13} \mathrm{C}$ NMR (101 MHz, $\mathrm{CDCl}_{3}$ )

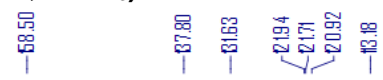

第

$\mathrm{SO}_{2} \mathrm{~F}$

$2 q$

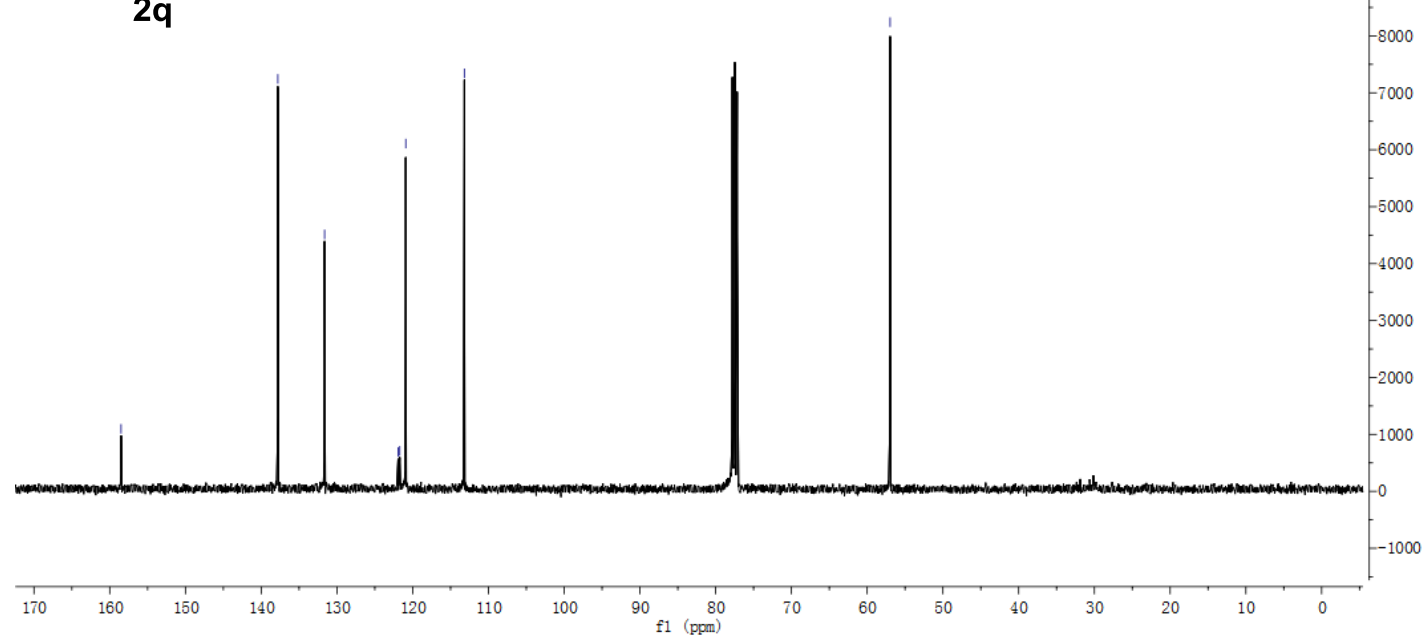


${ }^{19}$ F NMR (376 MHz, $\mathrm{CDCl}_{3}$ )

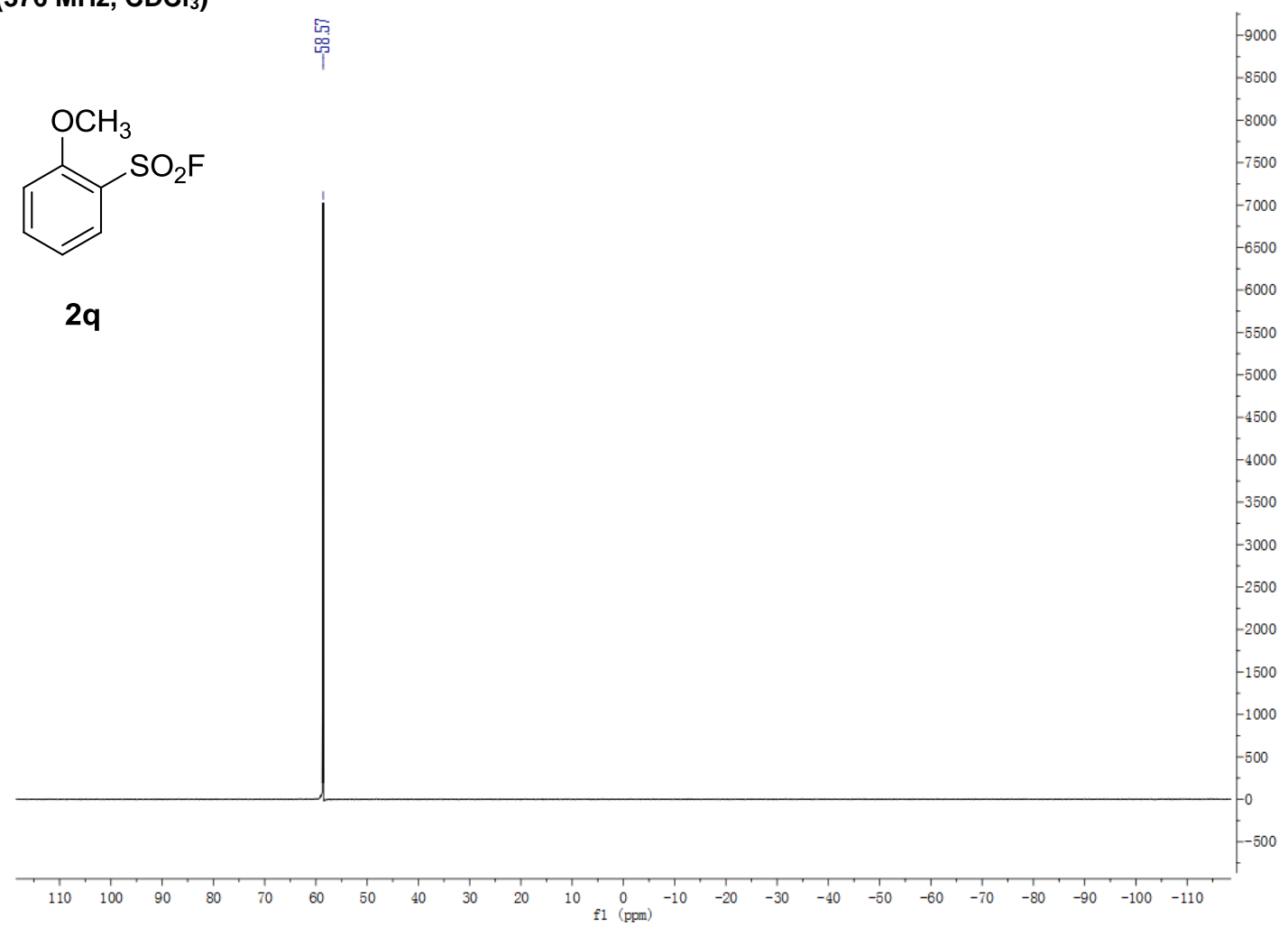

${ }^{1} \mathrm{H}$ NMR (400 MHz, $\mathrm{CDCl}_{3}$ )

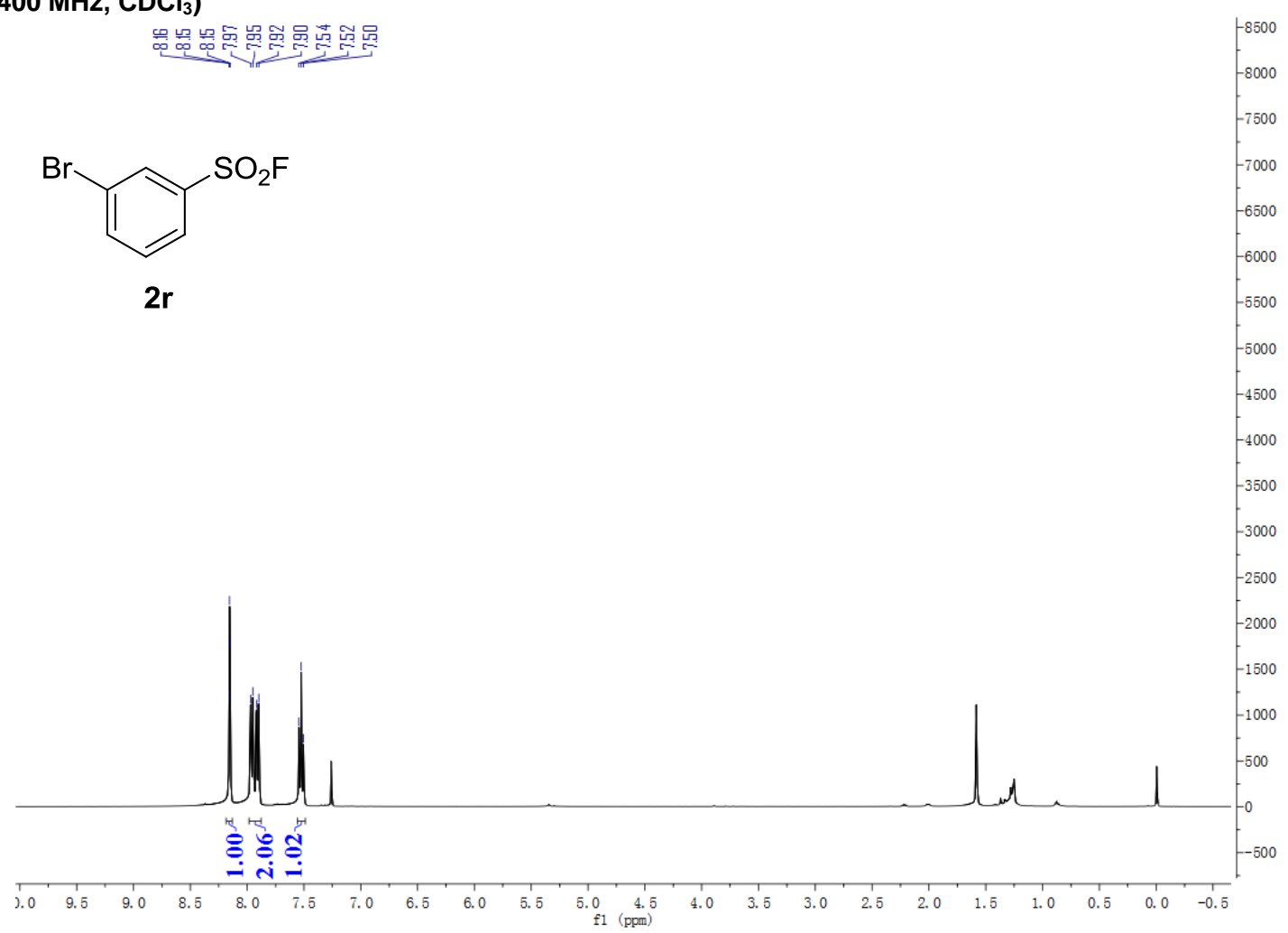


${ }^{13} \mathrm{C}$ NMR (101 MHz, $\mathrm{CDCl}_{3}$ )

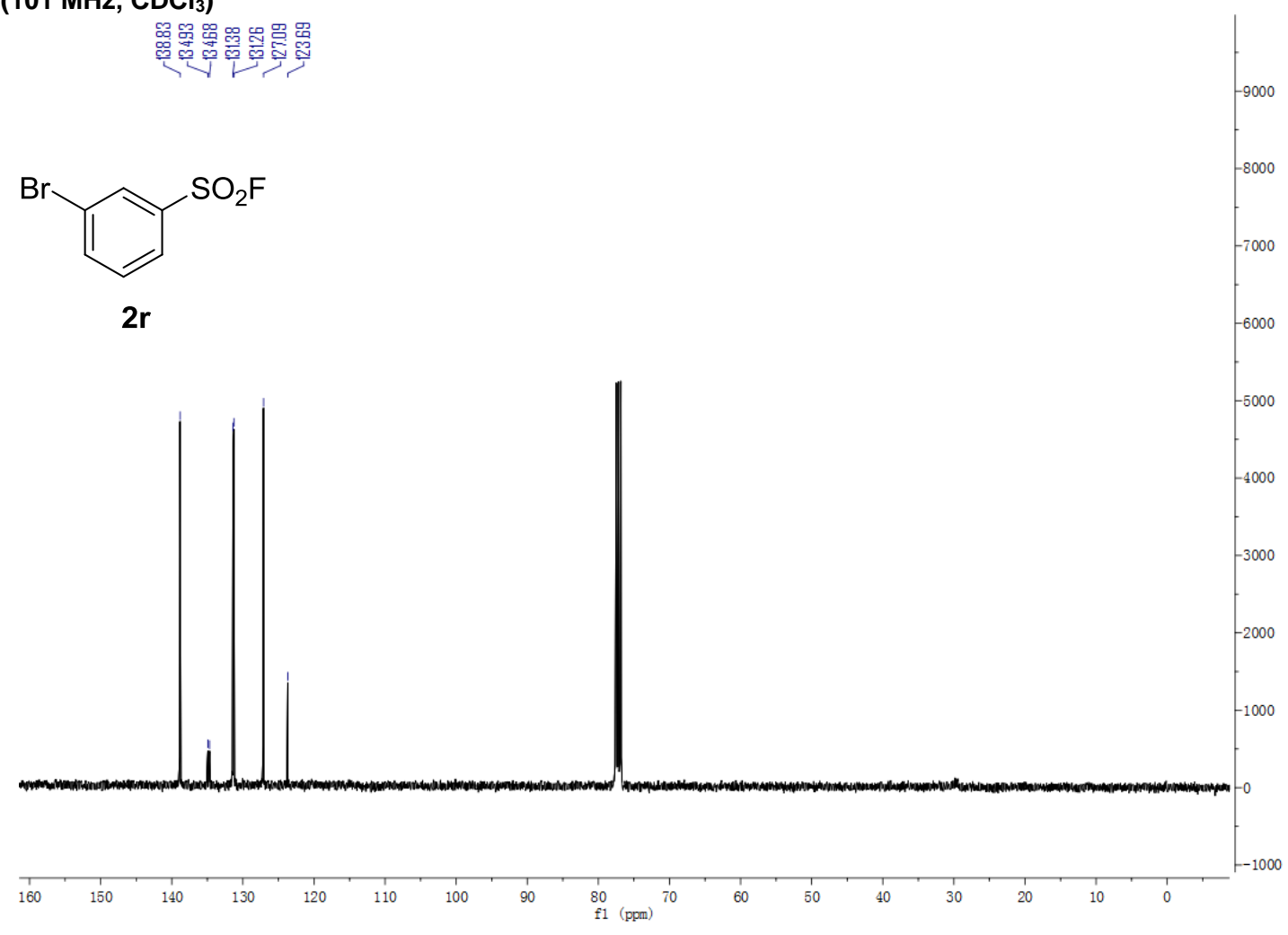

${ }^{19} \mathrm{~F} \mathrm{NMR} \mathrm{(376} \mathrm{MHz,} \mathrm{CDCl}_{3}$ )

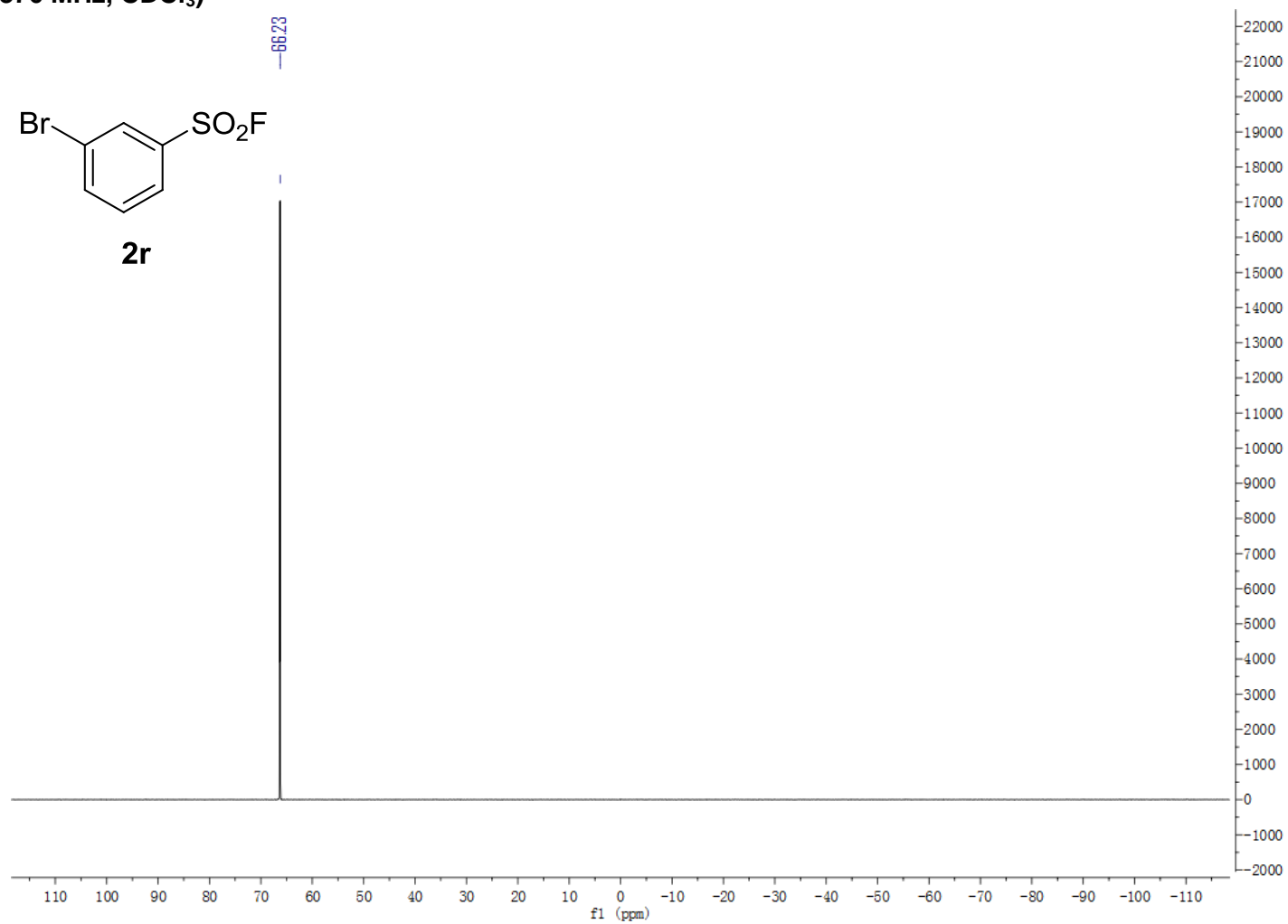


${ }^{1} \mathrm{H}$ NMR $\left(400 \mathrm{MHz}, \mathrm{CDCl}_{3}\right)$

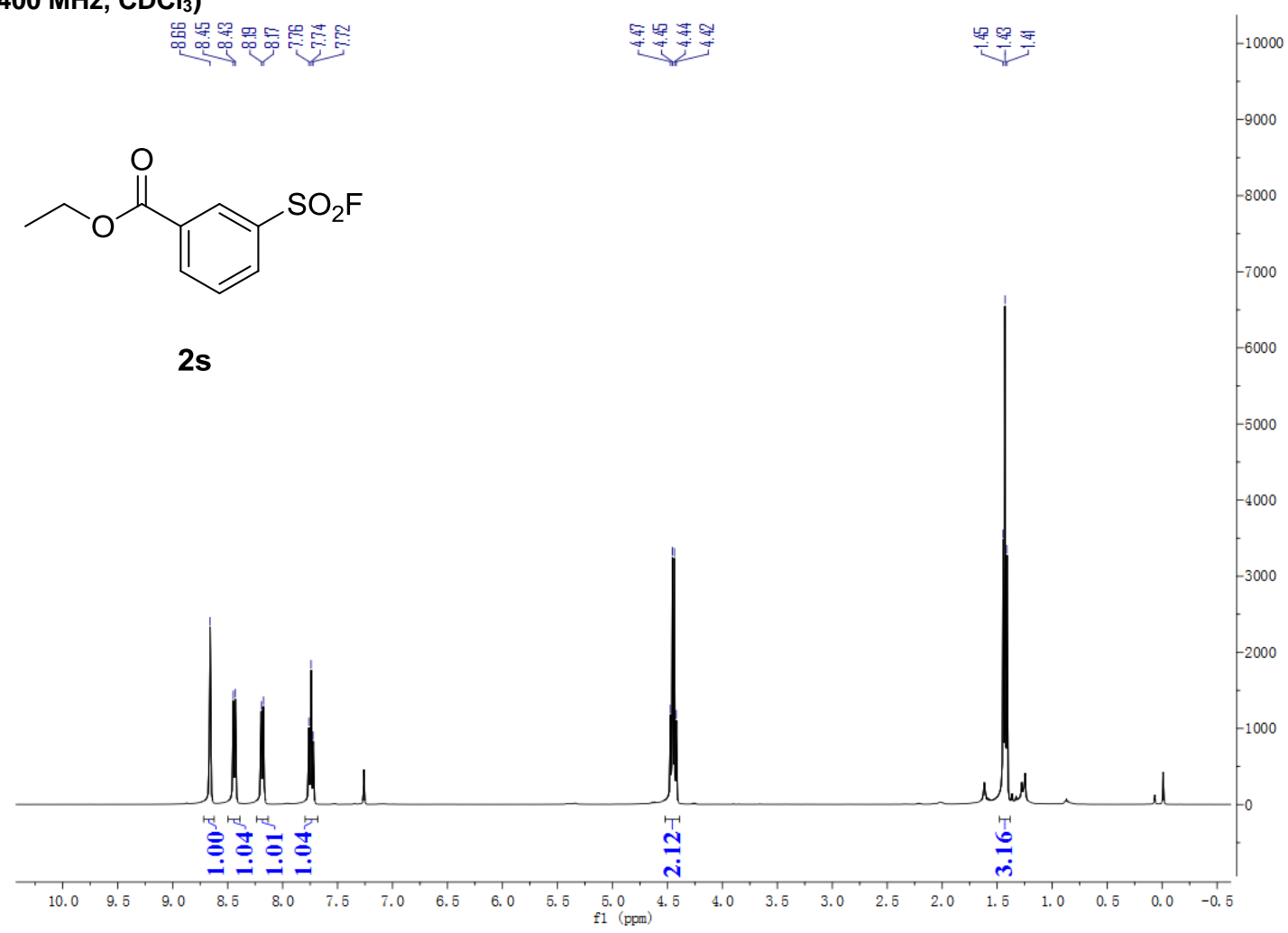

${ }^{13} \mathrm{C}$ NMR (101 MHz, $\mathrm{CDCl}_{3}$ )

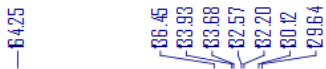<smiles>CCOC(=O)c1cccc(S(=O)(=O)F)c1</smiles>

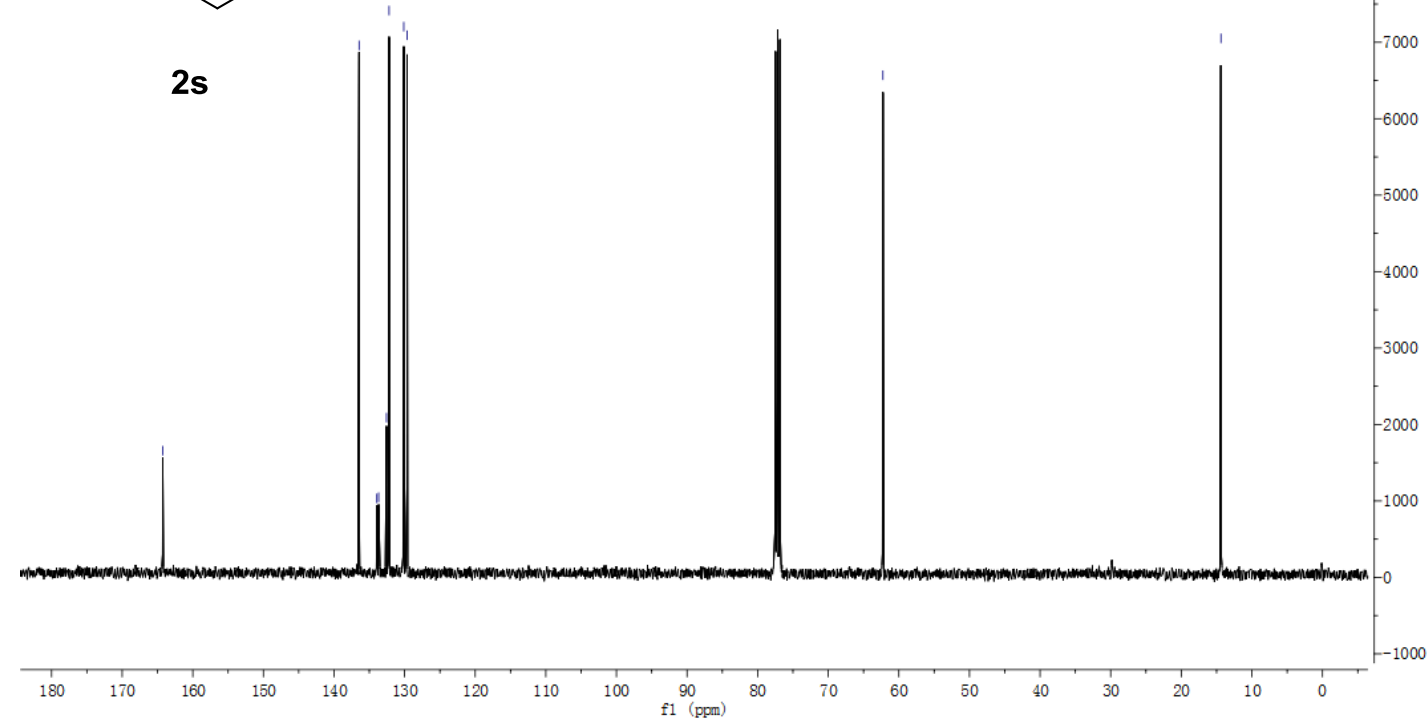


$\left.{ }^{19} \mathrm{~F} \mathrm{NMR} \mathrm{(376} \mathrm{MHz,} \mathrm{CDCl}_{3}\right)$

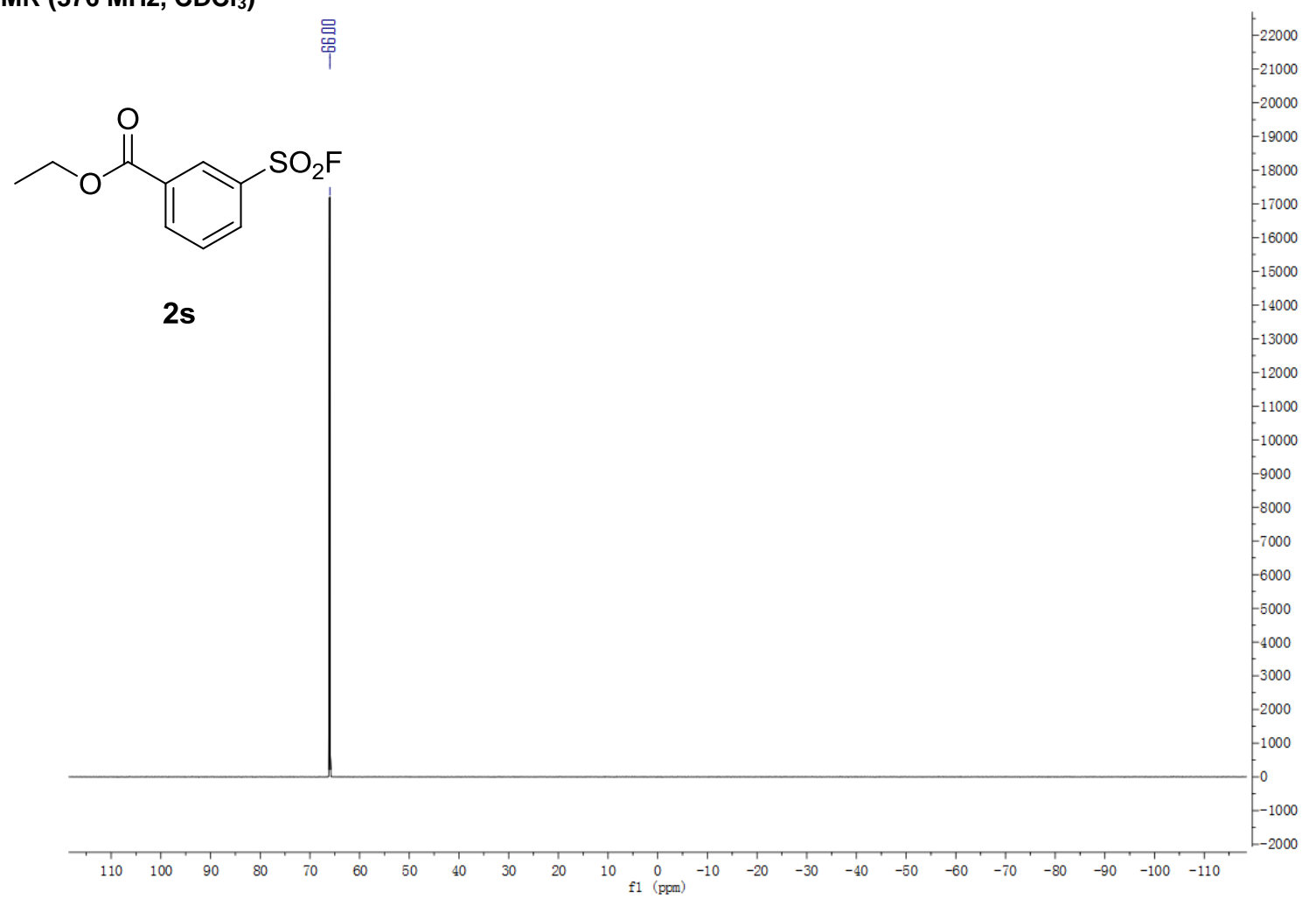

${ }^{1} \mathrm{H}$ NMR $\left(400 \mathrm{MHz}, \mathrm{CDCl}_{3}\right)$

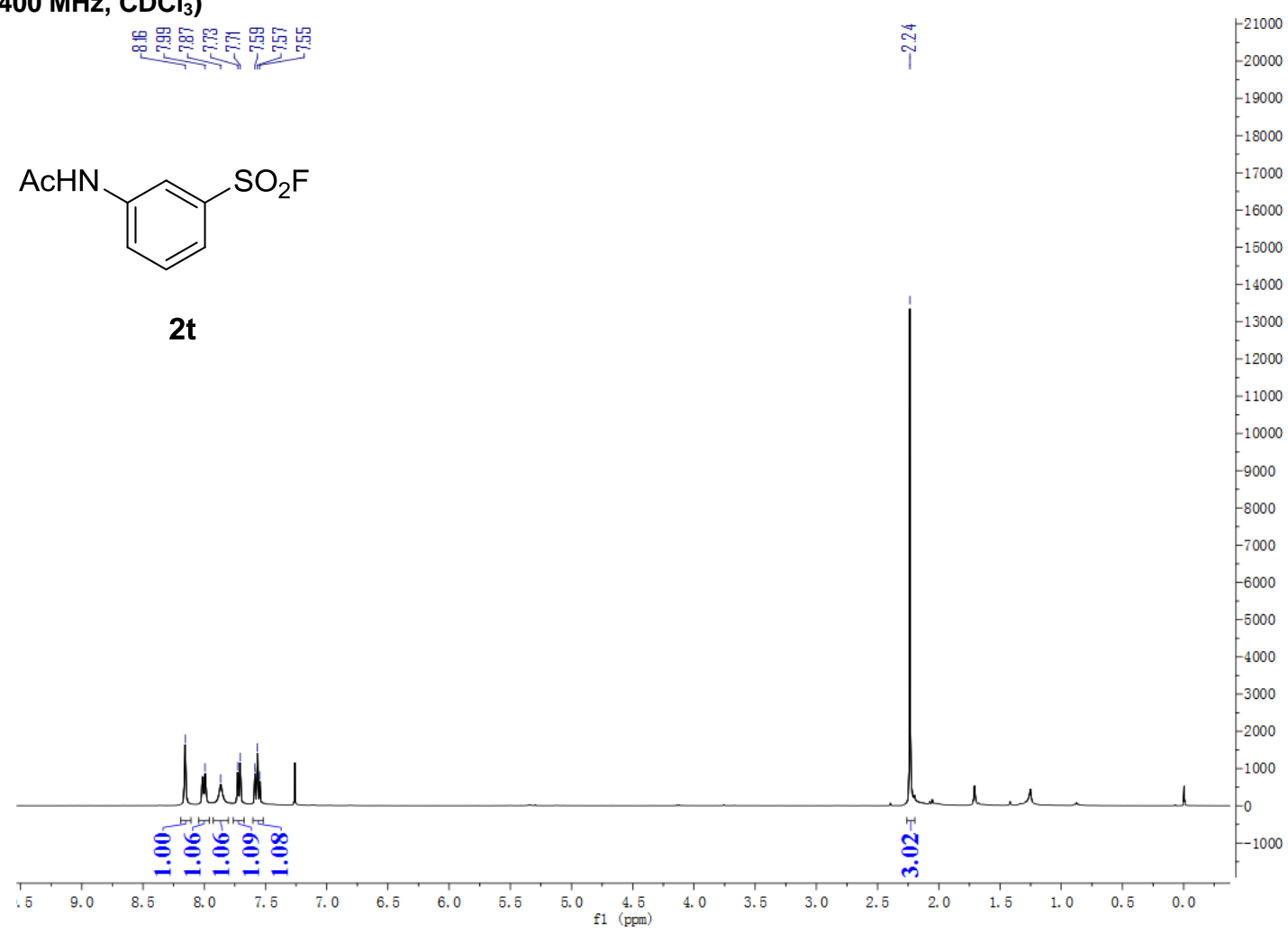


${ }^{13} \mathrm{C}$ NMR (101 MHz, $\mathrm{CDCl}_{3}$ )

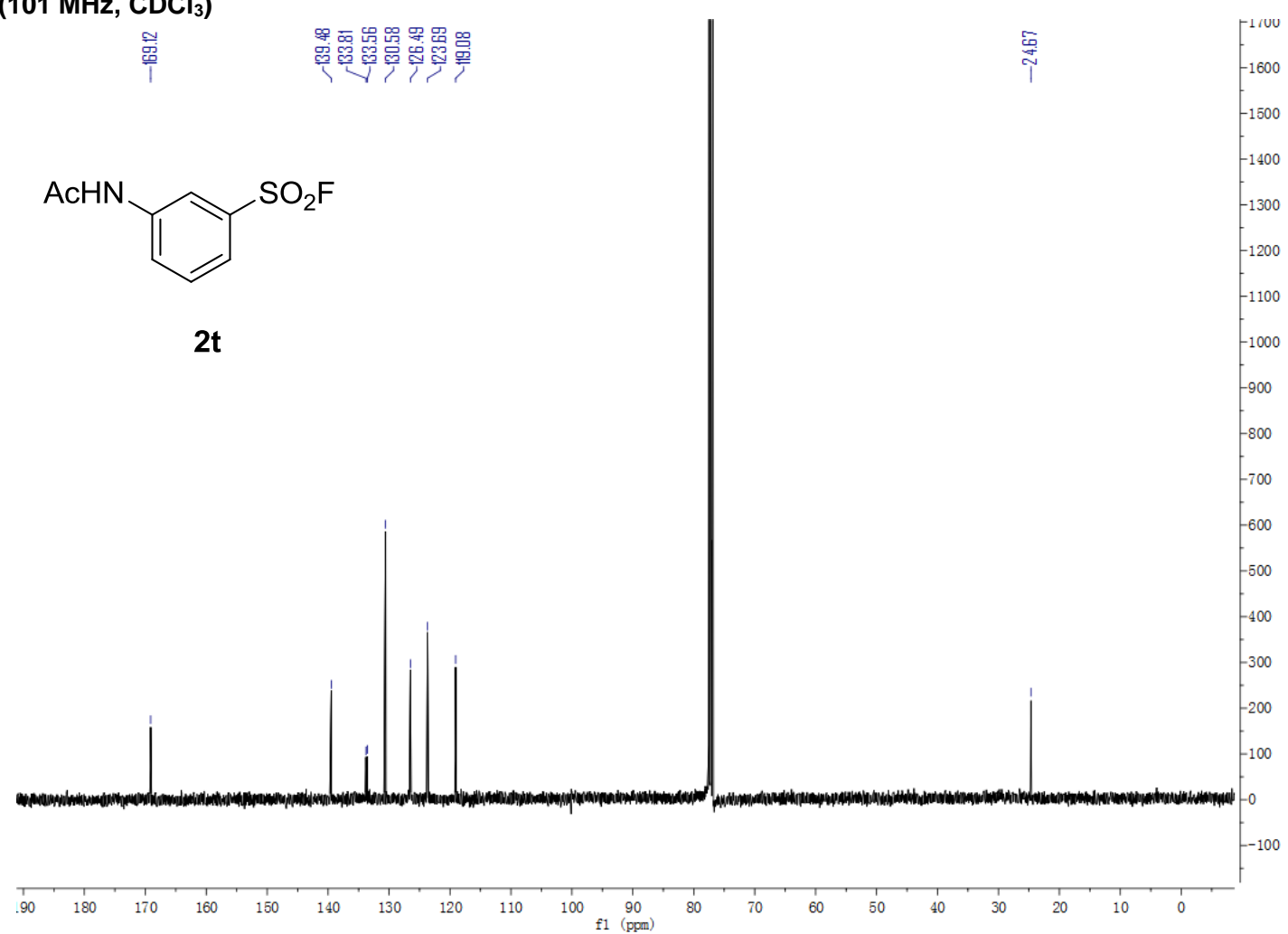

${ }^{19}$ F NMR (376 $\mathrm{MHz}, \mathrm{CDCl}_{3}$ )<smiles>CC(C)Nc1cccc(S(=O)(=O)F)c1</smiles>

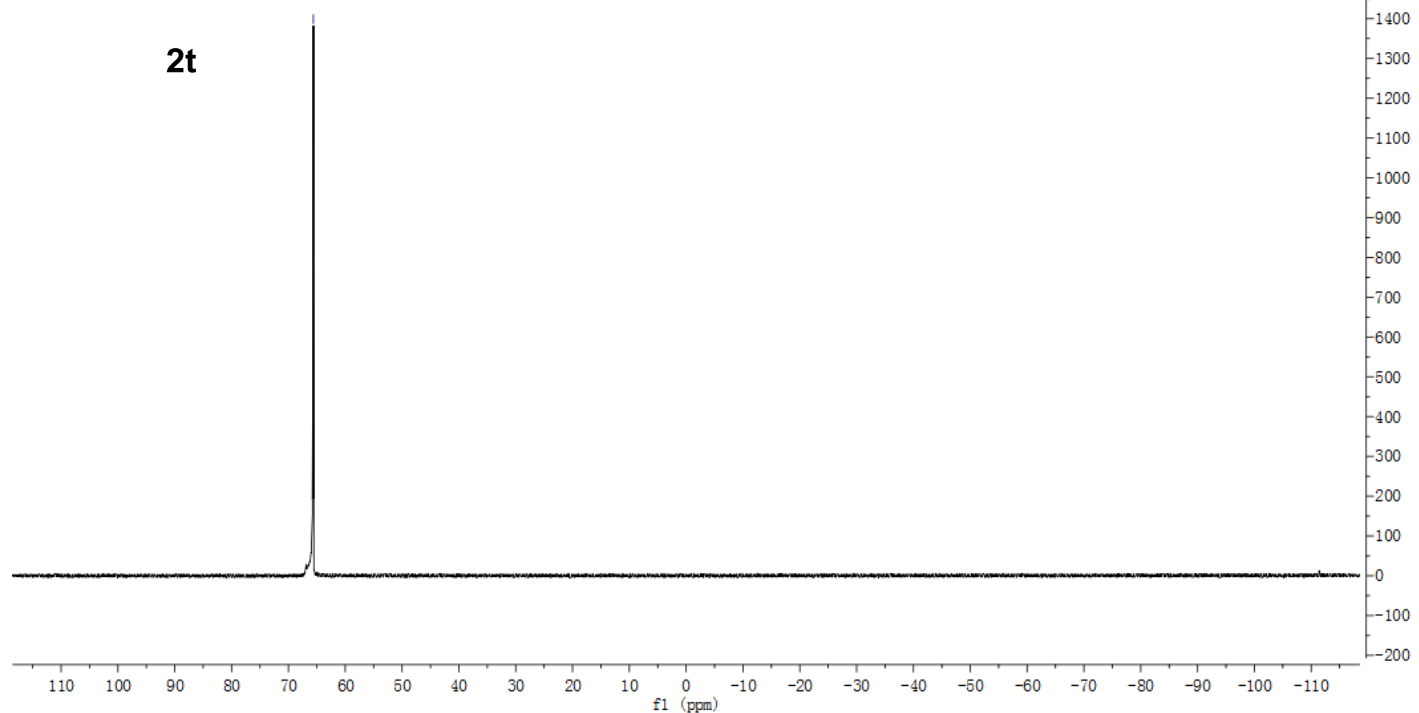


${ }^{1} \mathrm{H}$ NMR $\left(400 \mathrm{MHz}, \mathrm{CDCl}_{3}\right)$

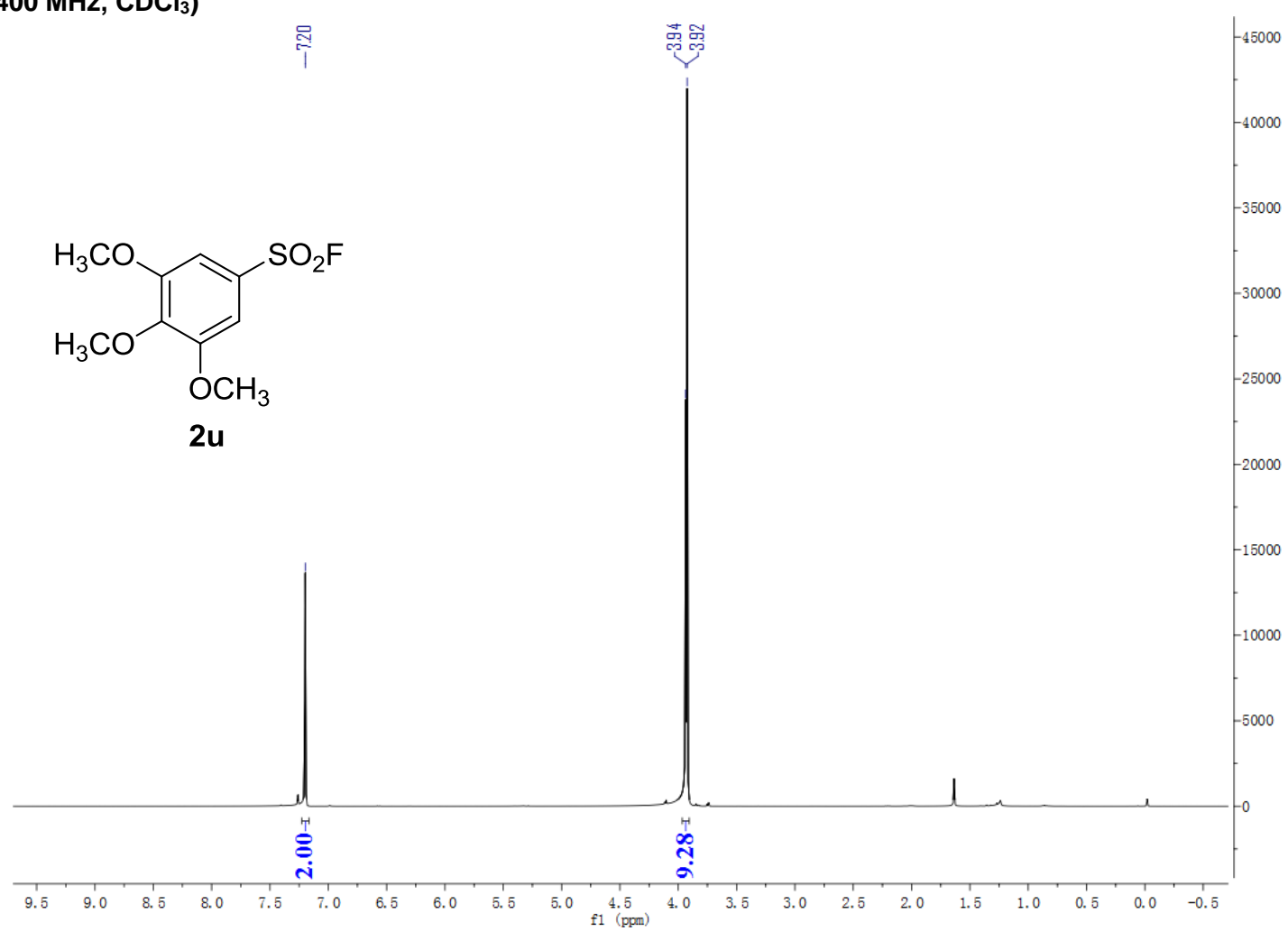

${ }^{13} \mathrm{C}$ NMR (101 MHz, $\mathrm{CDCl}_{3}$ )

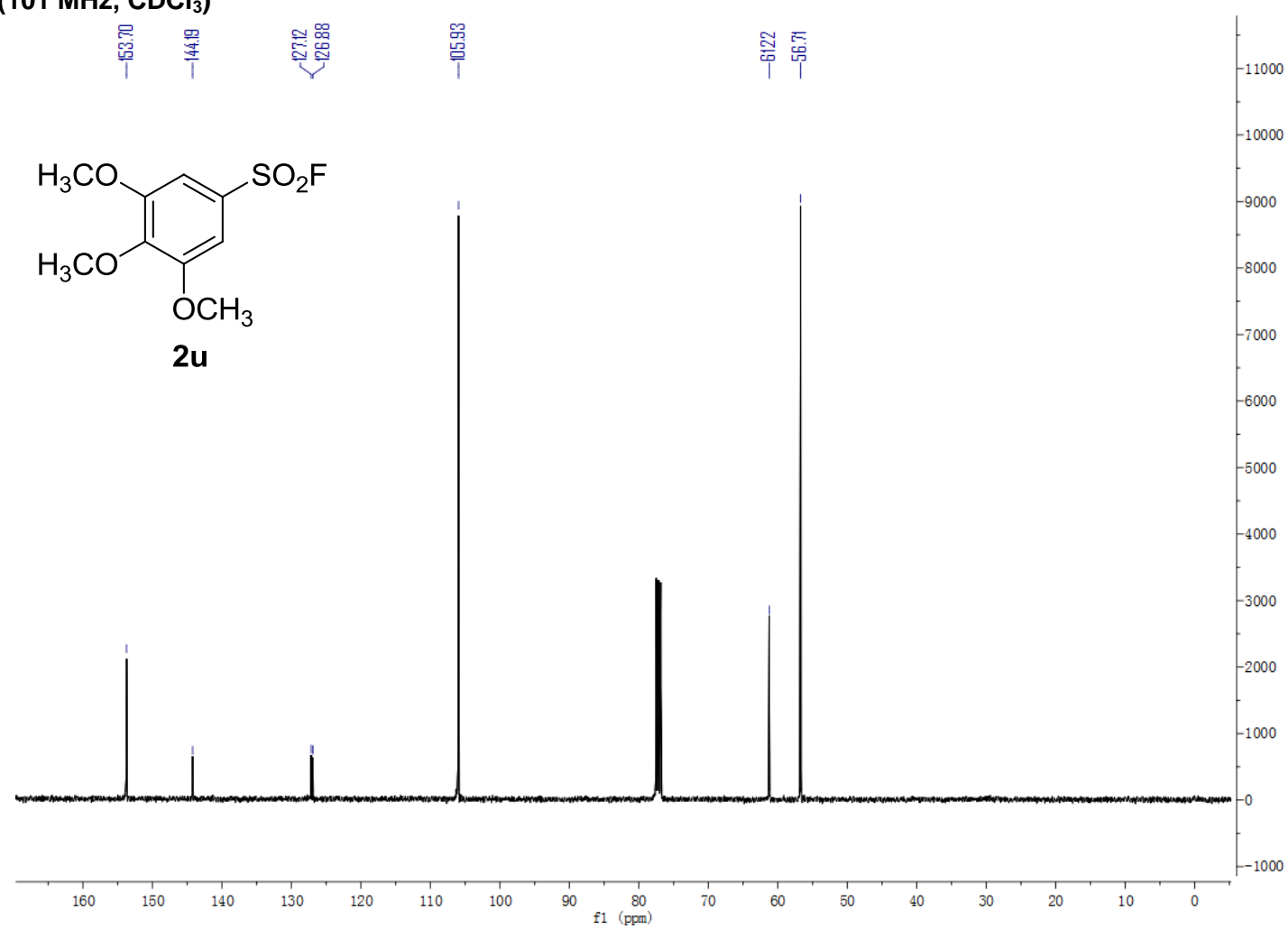


${ }^{19}$ F NMR (376 MHz, $\mathrm{CDCl}_{3}$ )

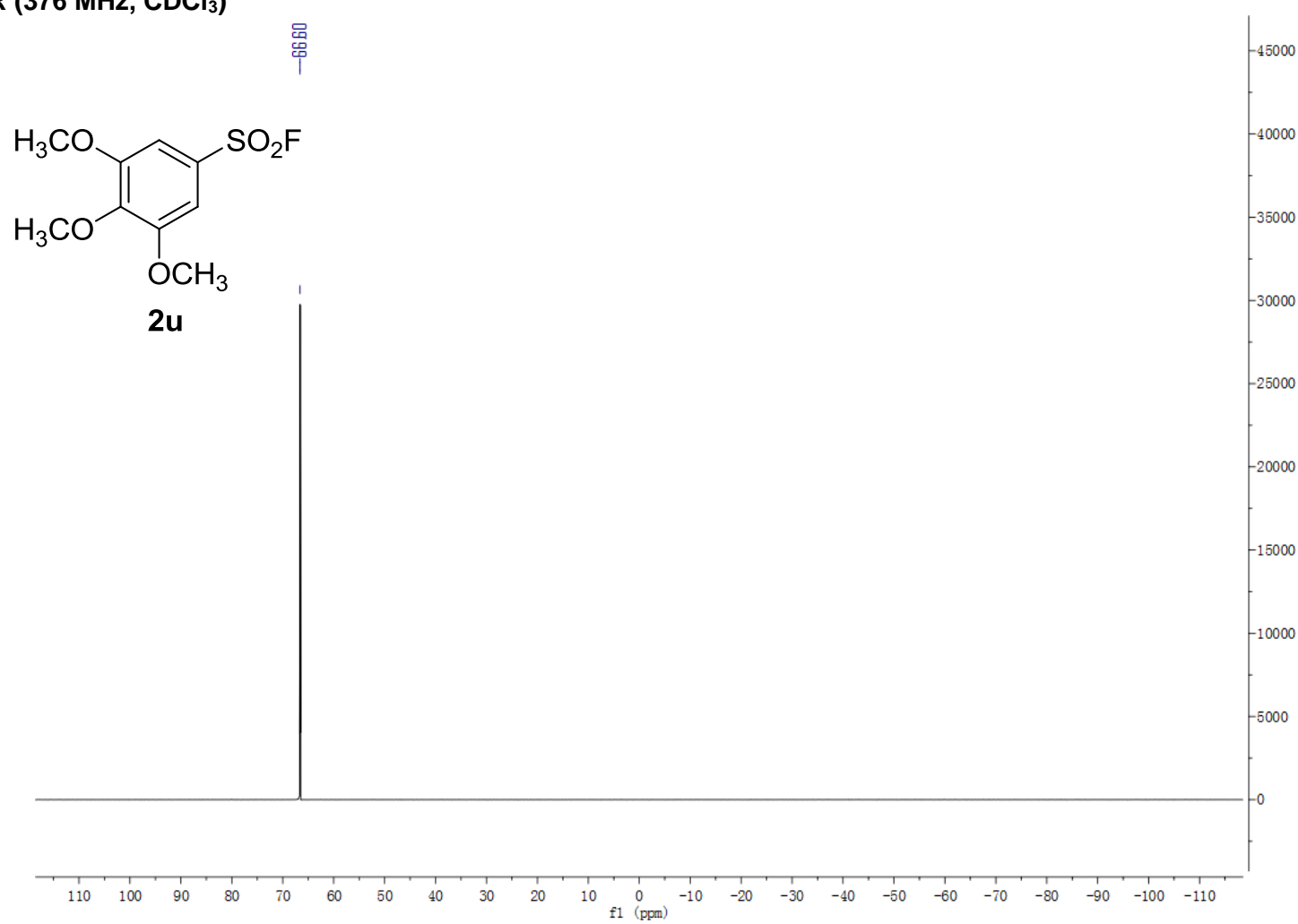

${ }^{1} \mathrm{H}$ NMR (400 MHz, $\mathrm{CDCl}_{3}$ )

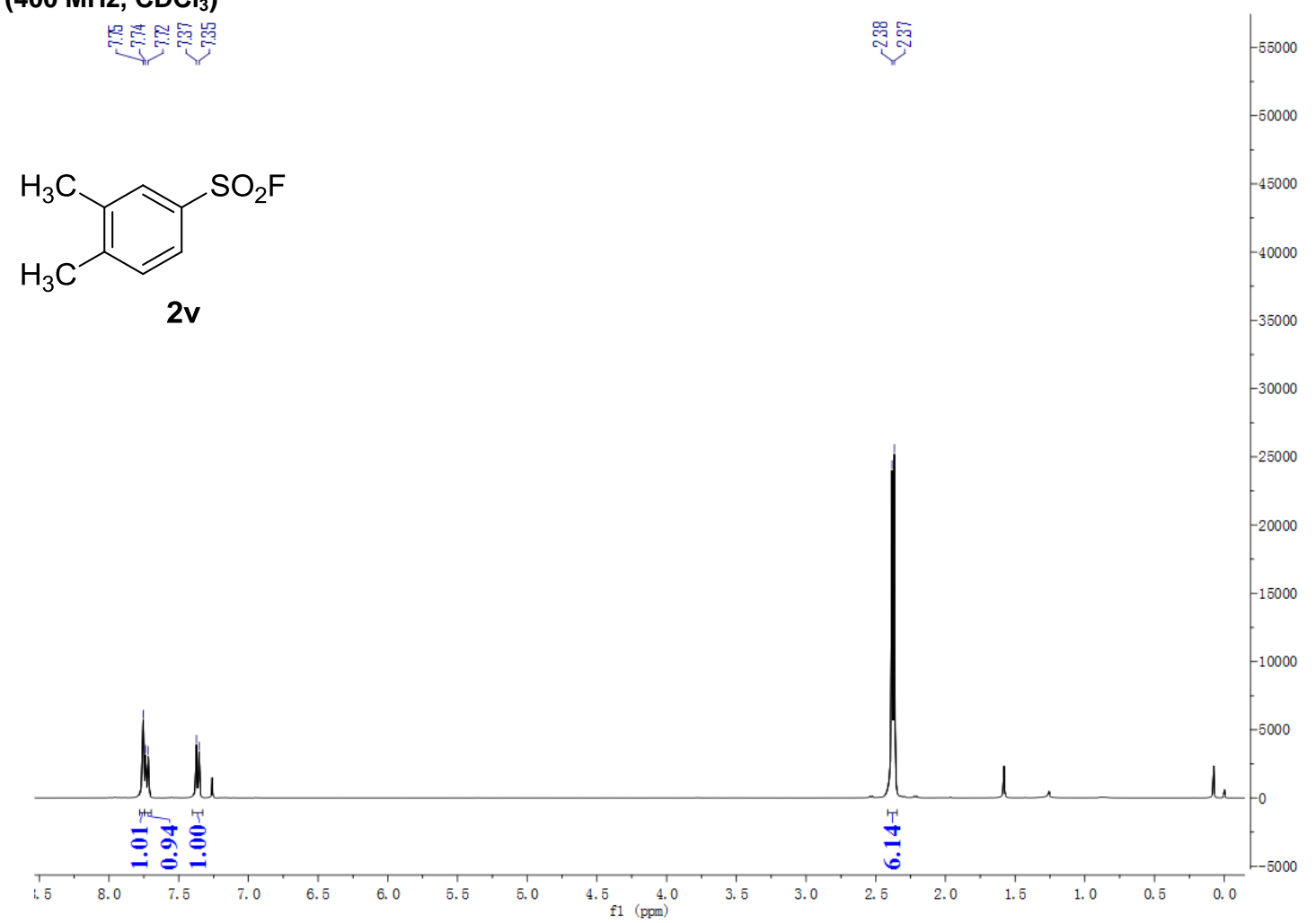


${ }^{13} \mathrm{C}$ NMR (126 MHz, $\mathrm{CDCl}_{3}$ )

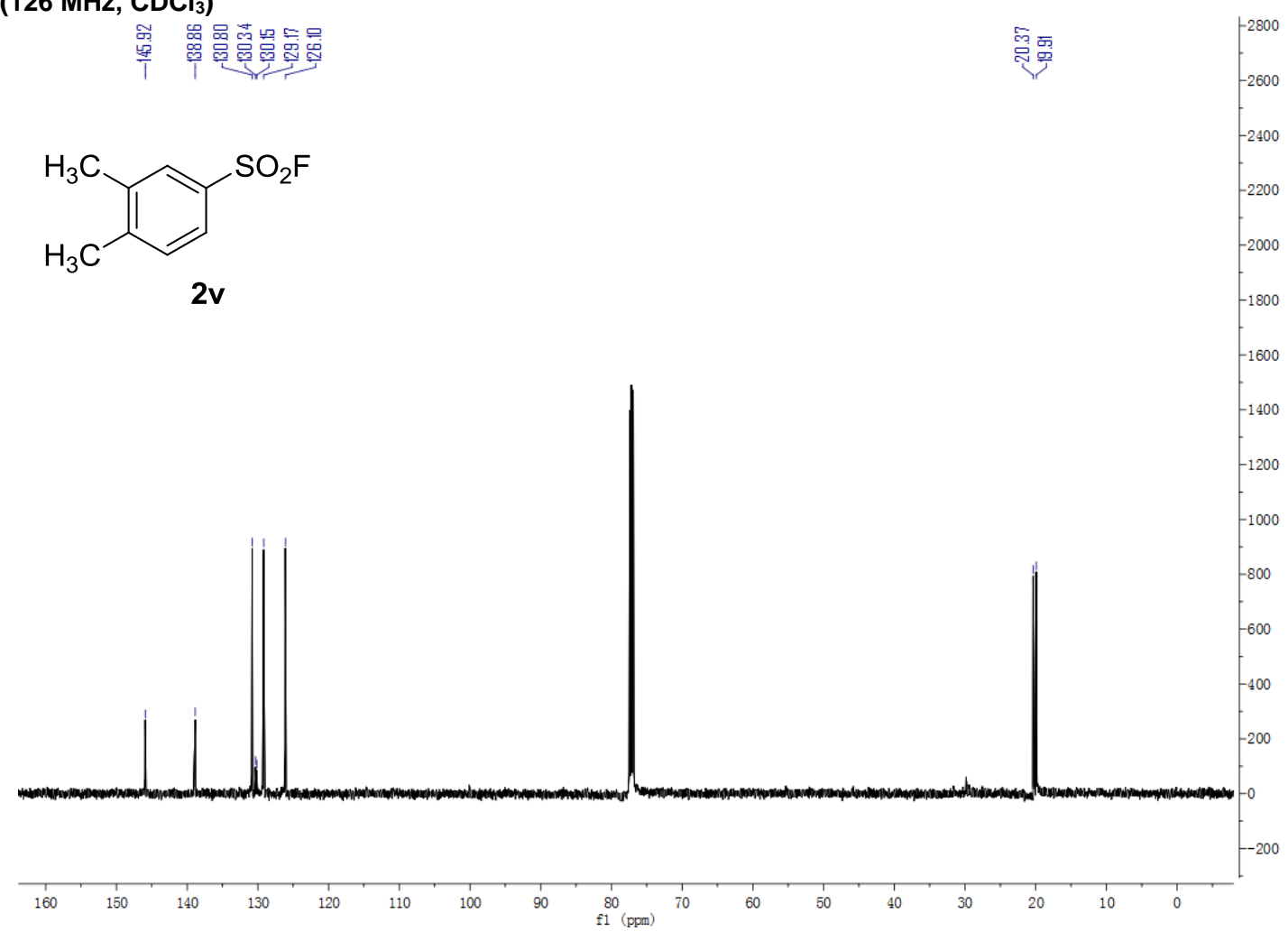

${ }^{19} \mathrm{~F} \mathrm{NMR}\left(376 \mathrm{MHz}, \mathrm{CDCl}_{3}\right.$ )<smiles>Cc1ccc(S(=O)(=O)F)cc1C</smiles>

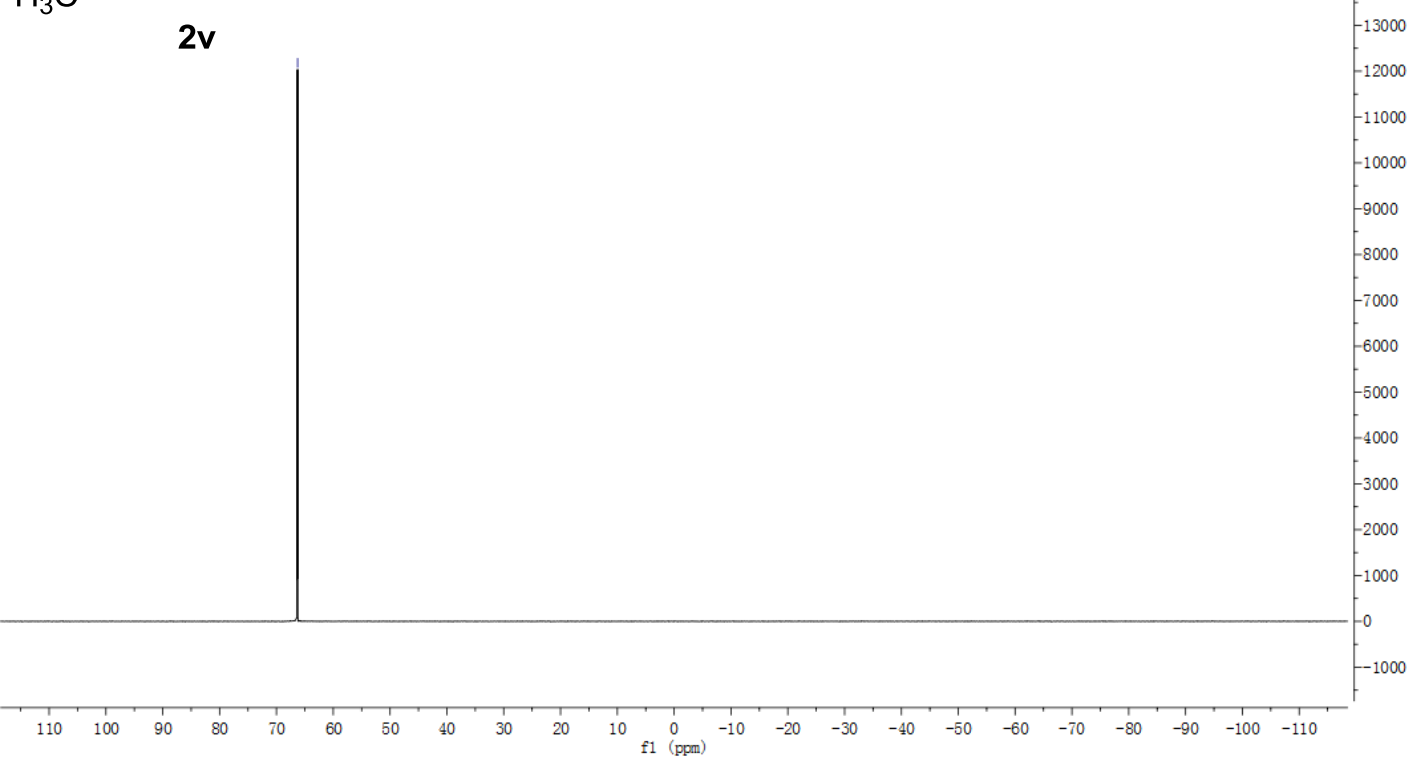


${ }^{1} \mathrm{H}$ NMR $\left(400 \mathrm{MHz}, \mathrm{CDCl}_{3}\right)$

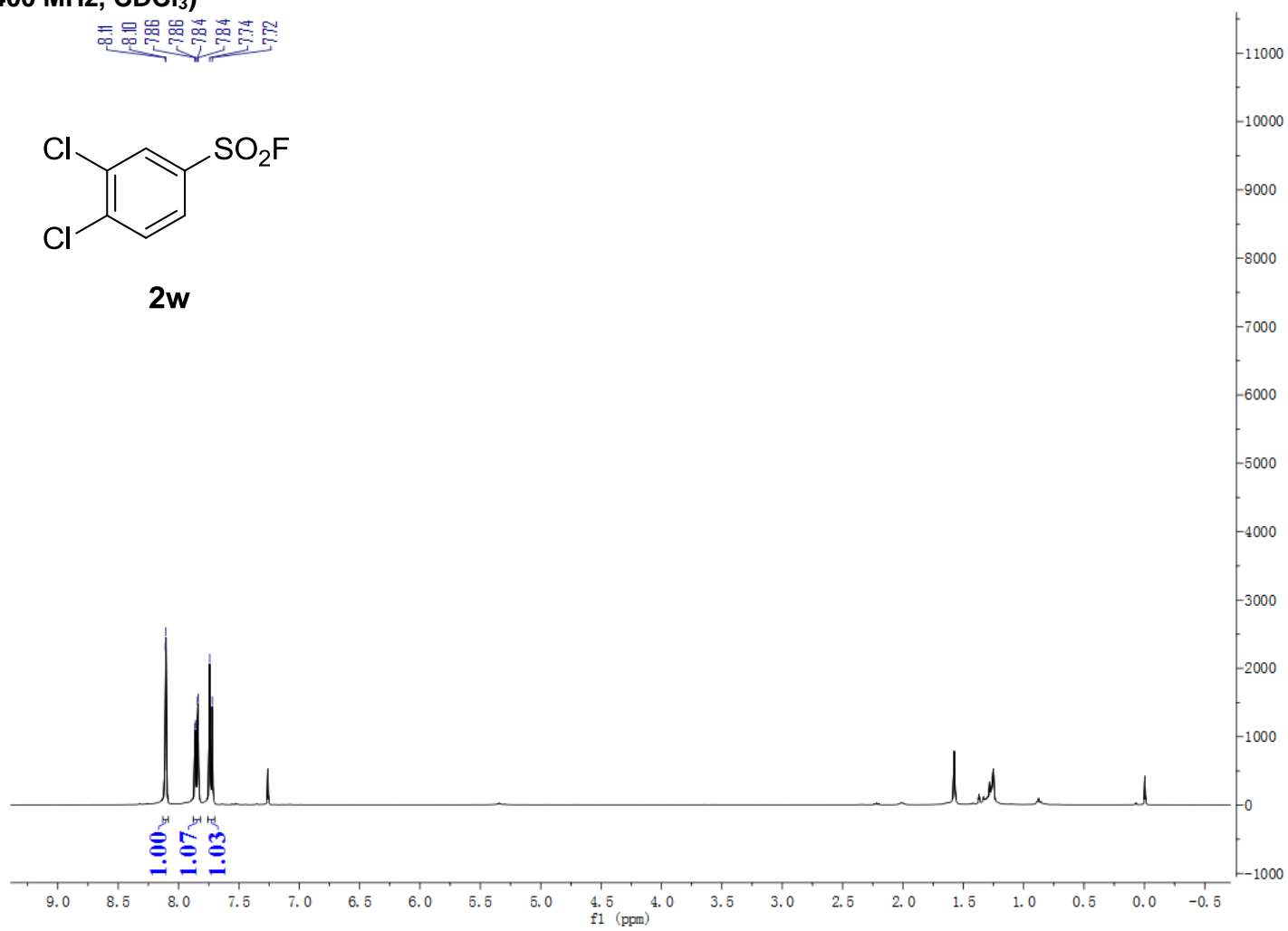

${ }^{13} \mathrm{C} \mathrm{NMR} \mathrm{(101} \mathrm{MHz,} \mathrm{CDCl}_{3}$ )

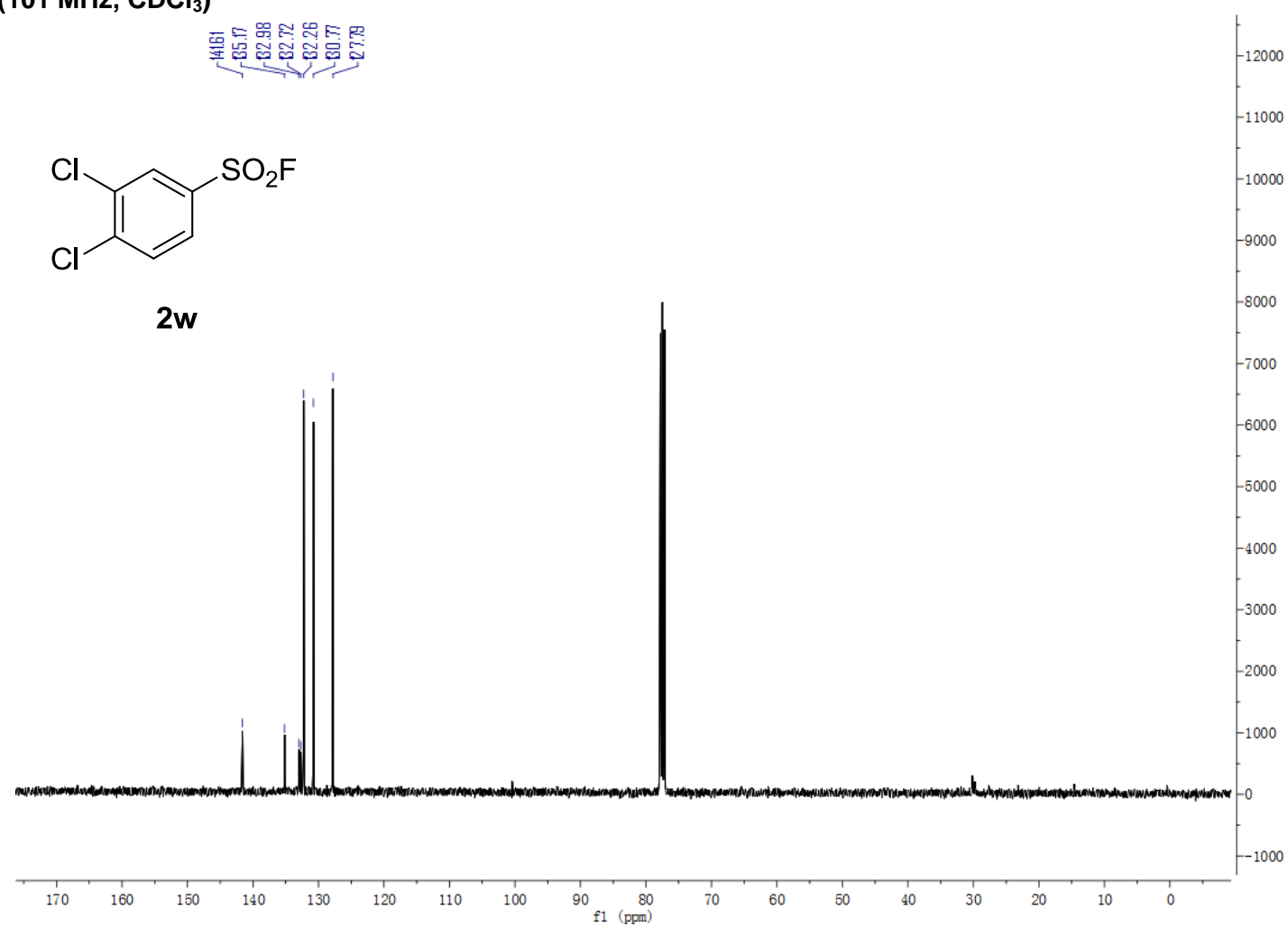


${ }^{19}$ F NMR (376 $\left.\mathrm{MHz}, \mathrm{CDCl}_{3}\right)$

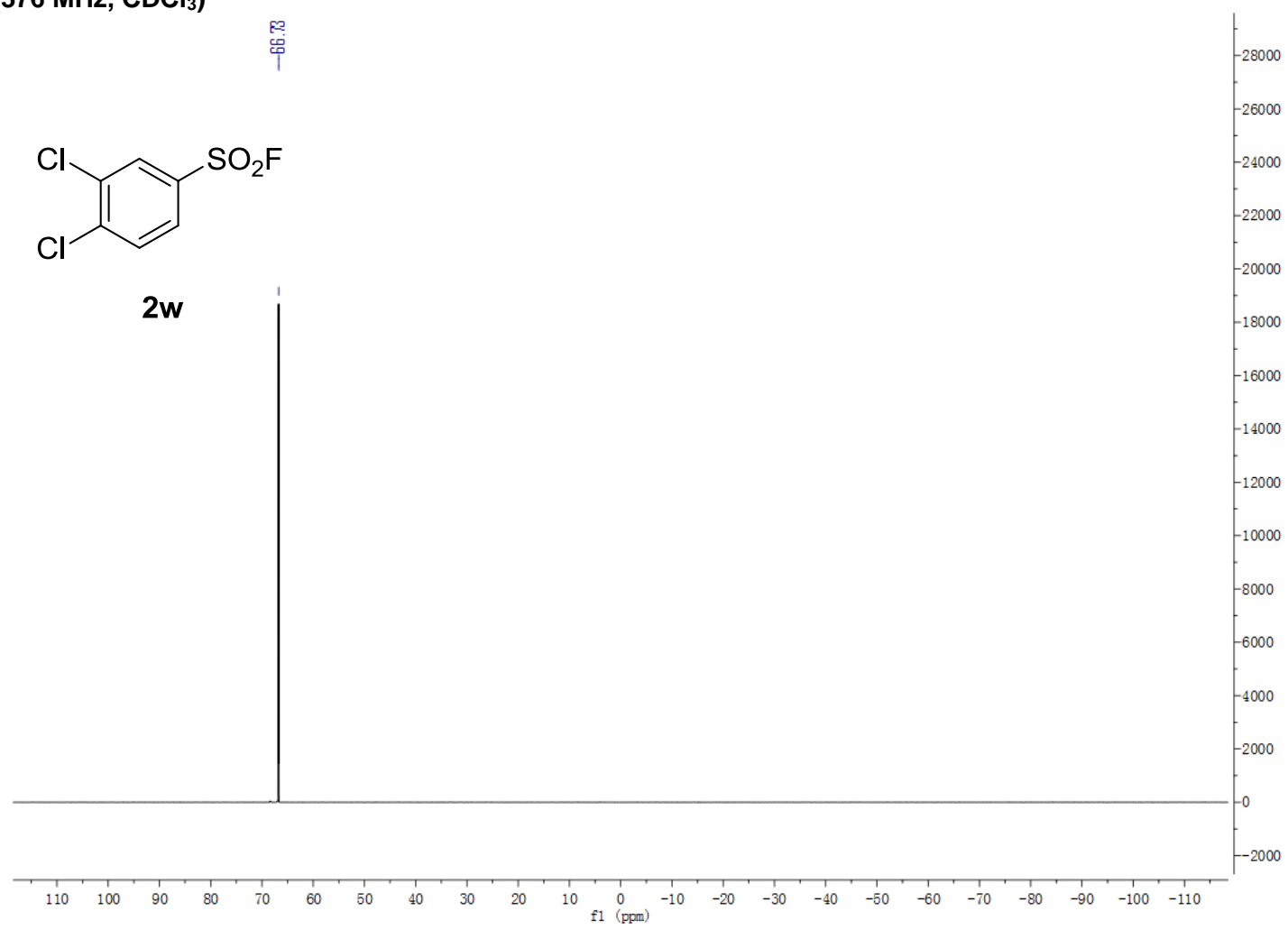

${ }^{1} \mathrm{H}$ NMR (400 MHz, $\left.\mathrm{CDCl}_{3}\right)$

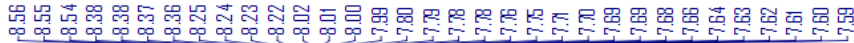<smiles>O=S(=O)(OF)c1cccc2ccccc12</smiles>

2x

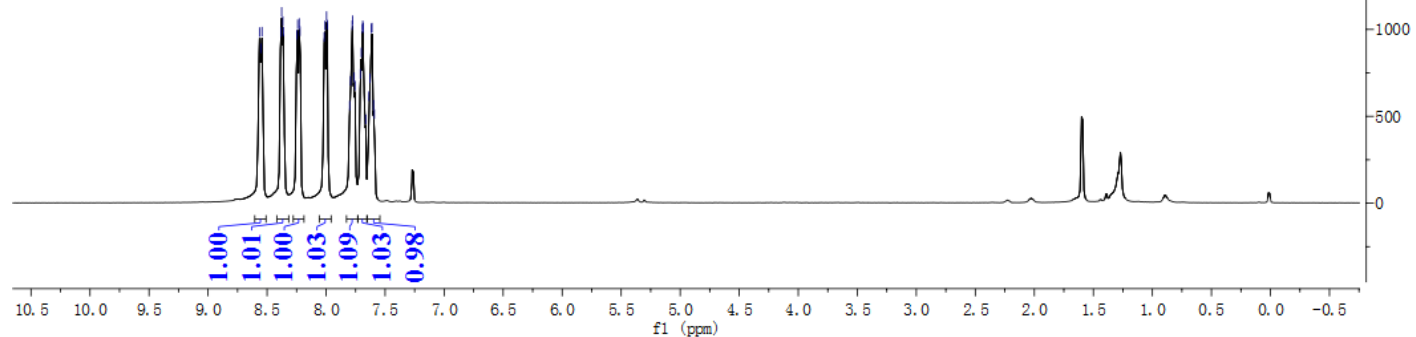


${ }^{13} \mathrm{C}$ NMR (101 MHz, $\mathrm{CDCl}_{3}$ )

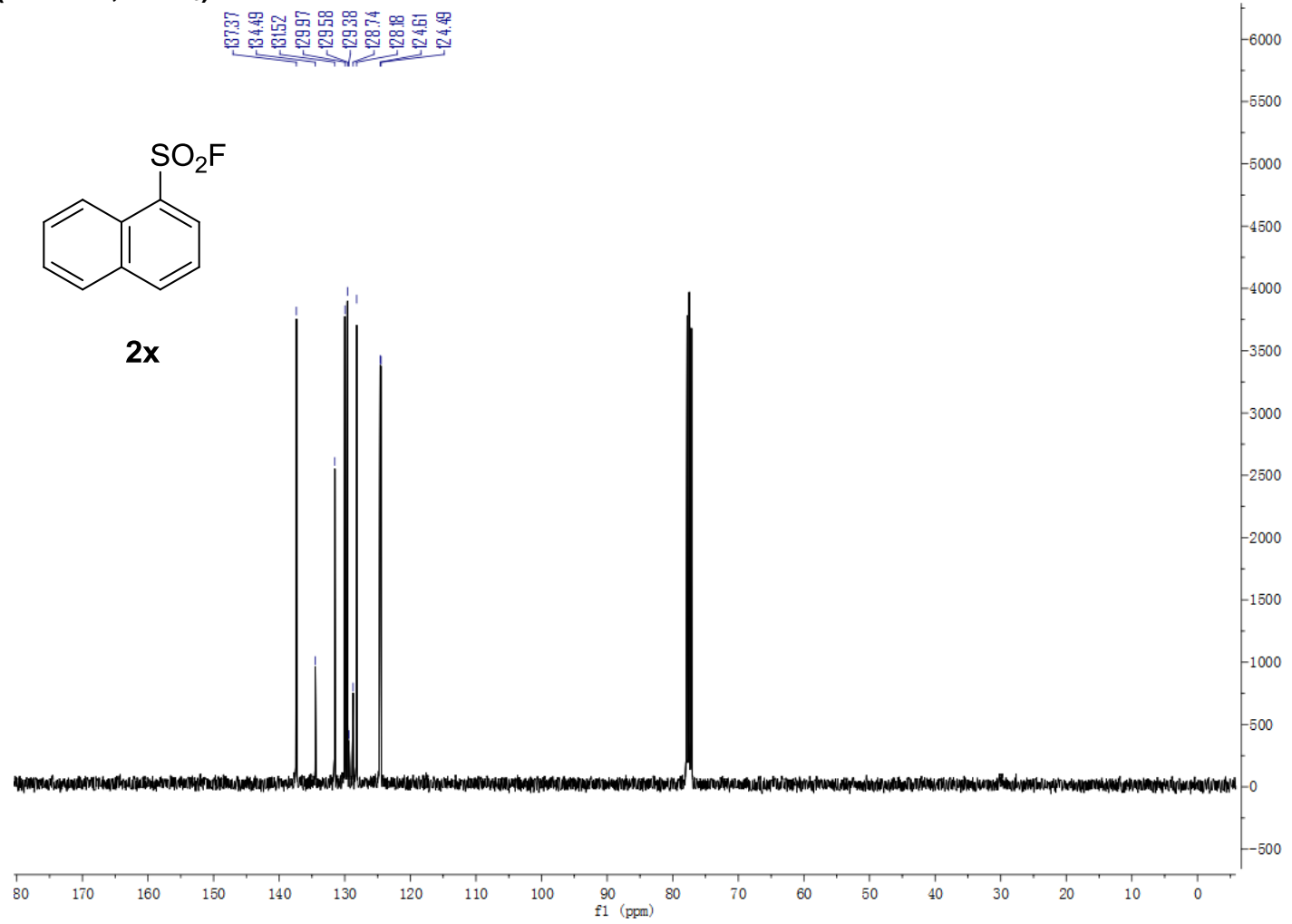

${ }^{19} \mathrm{~F} \mathrm{NMR}\left(376 \mathrm{MHz}, \mathrm{CDCl}_{3}\right.$ )

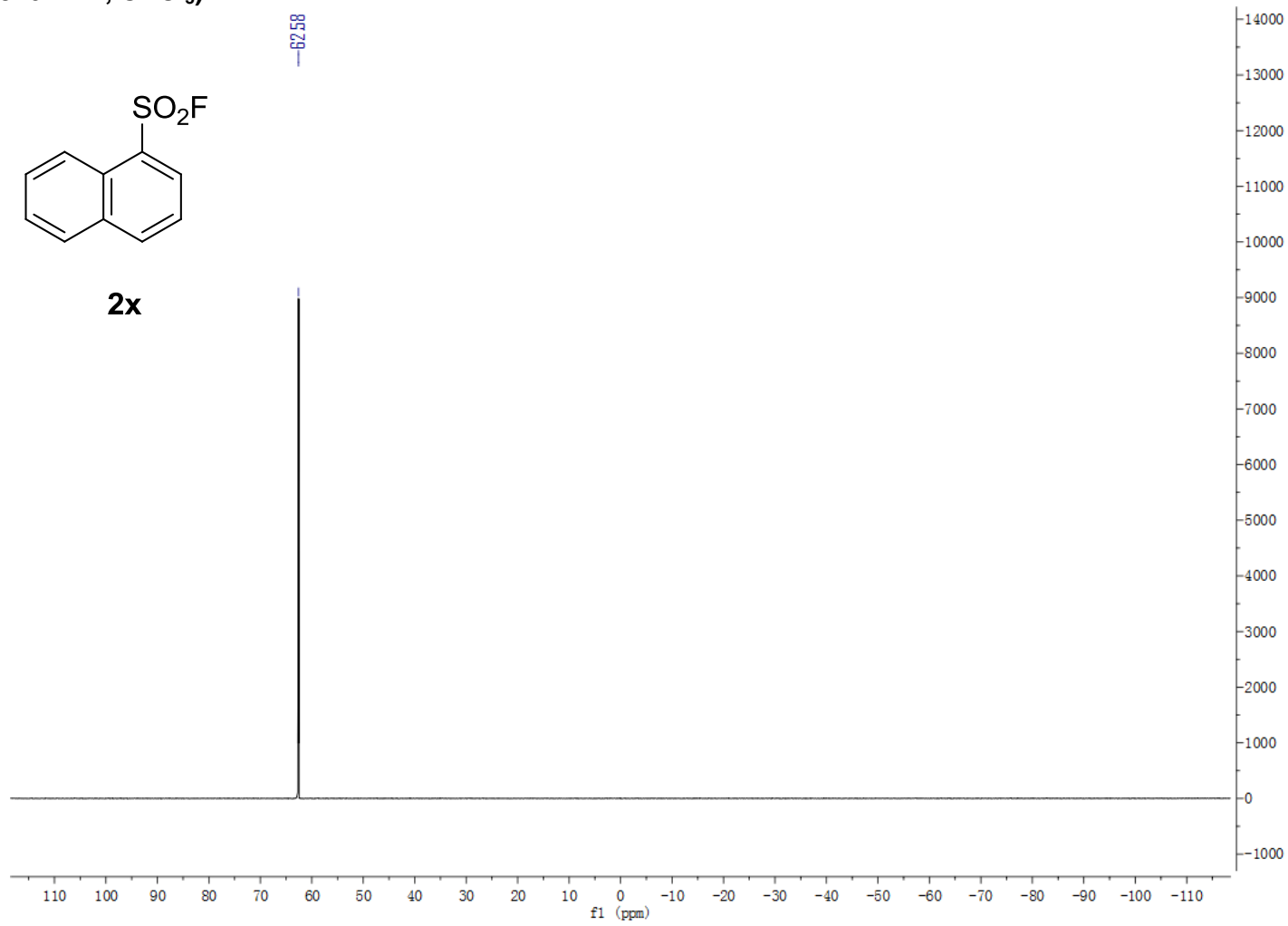


${ }^{1} \mathrm{H}$ NMR (400 MHz, $\mathrm{CDCl}_{3}$ )

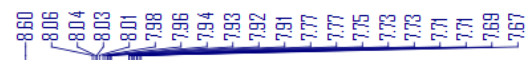<smiles>O=S(=O)(OF)c1ccc2ccccc2c1</smiles>

$2 y$

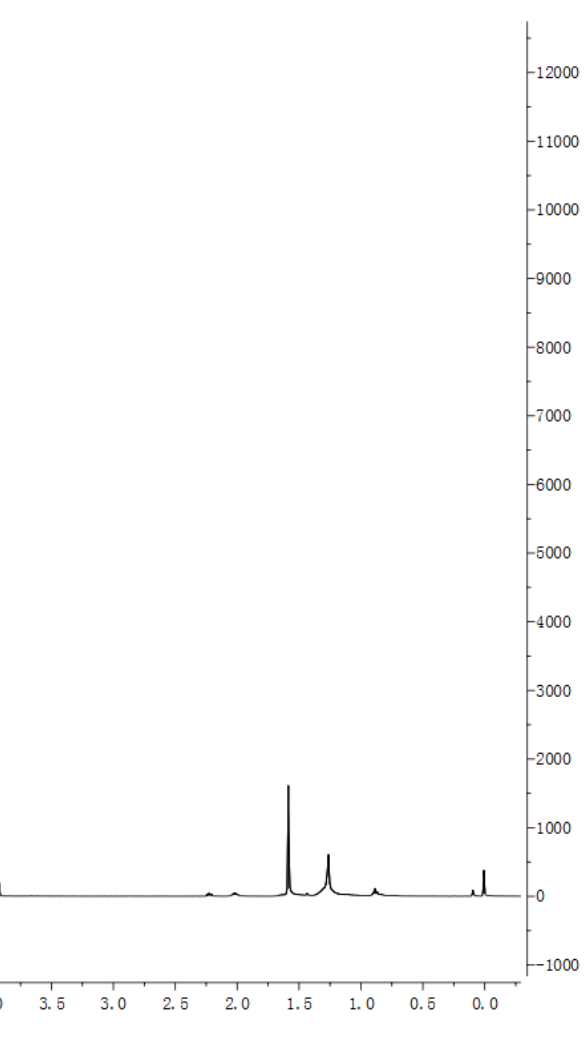

${ }^{13} \mathrm{C}$ NMR (126 $\mathrm{MHz}, \mathrm{CDCl}_{3}$ )

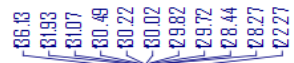<smiles>O=S(=O)(F)c1ccc2ccccc2c1</smiles>

$2 y$

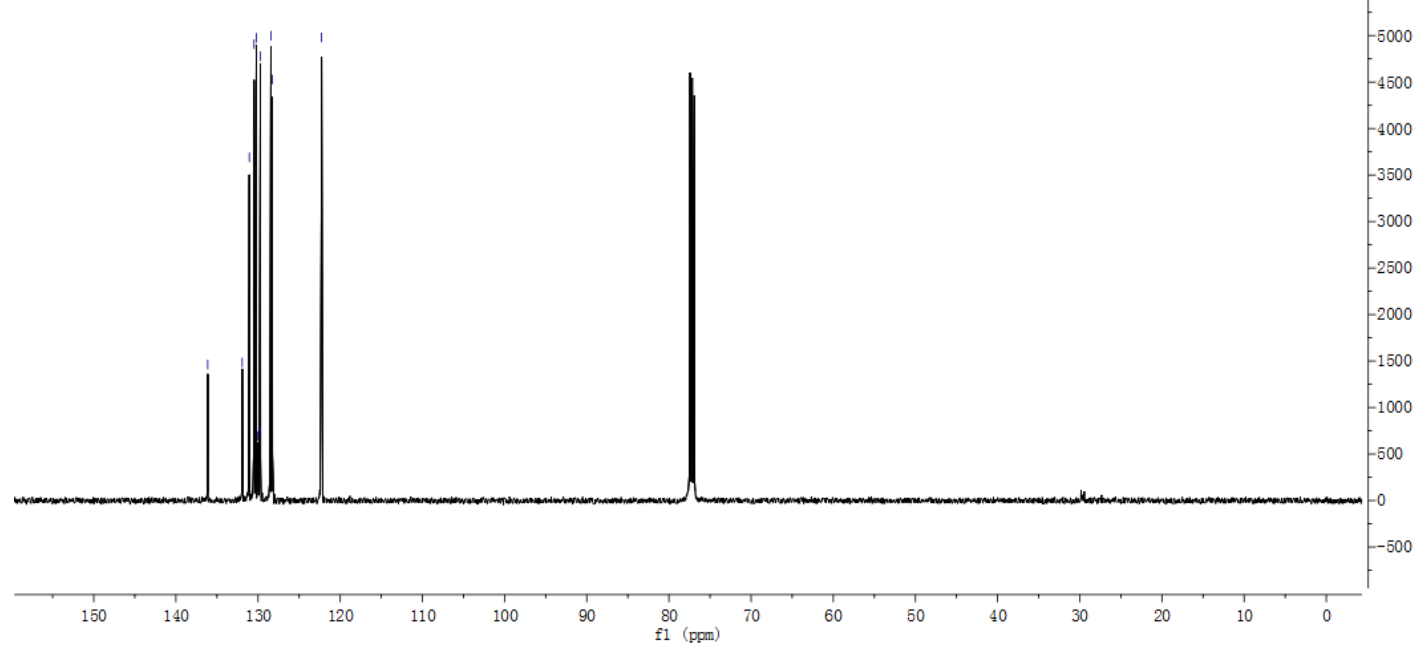


${ }^{19}$ F NMR (376 MHz, $\mathrm{CDCl}_{3}$ )

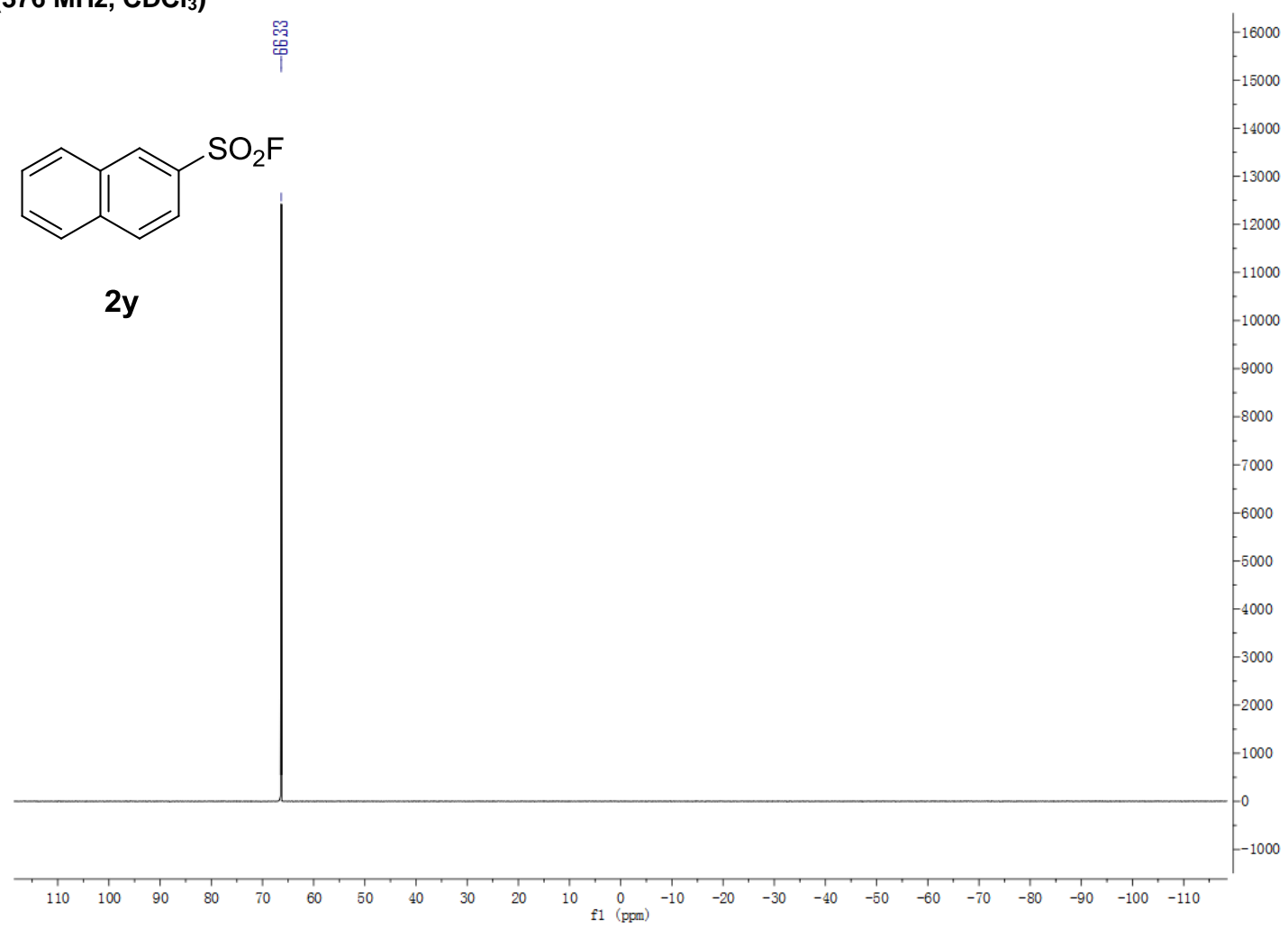

${ }^{1} \mathrm{H}$ NMR (400 MHz, $\mathrm{CDCl}_{3}$ )

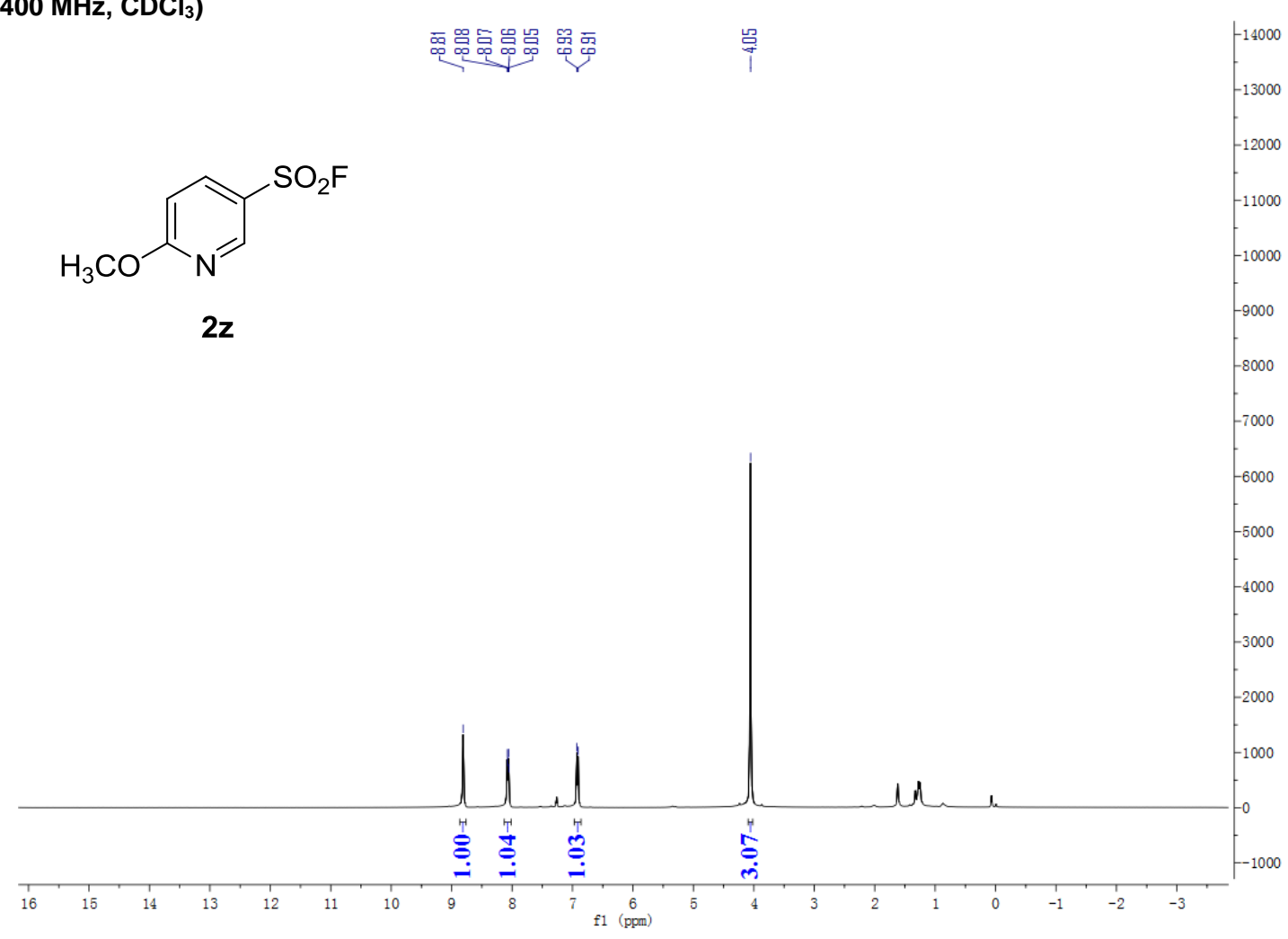


${ }^{13} \mathrm{C}$ NMR (101 MHz, $\left.\mathrm{CDCl}_{3}\right)$

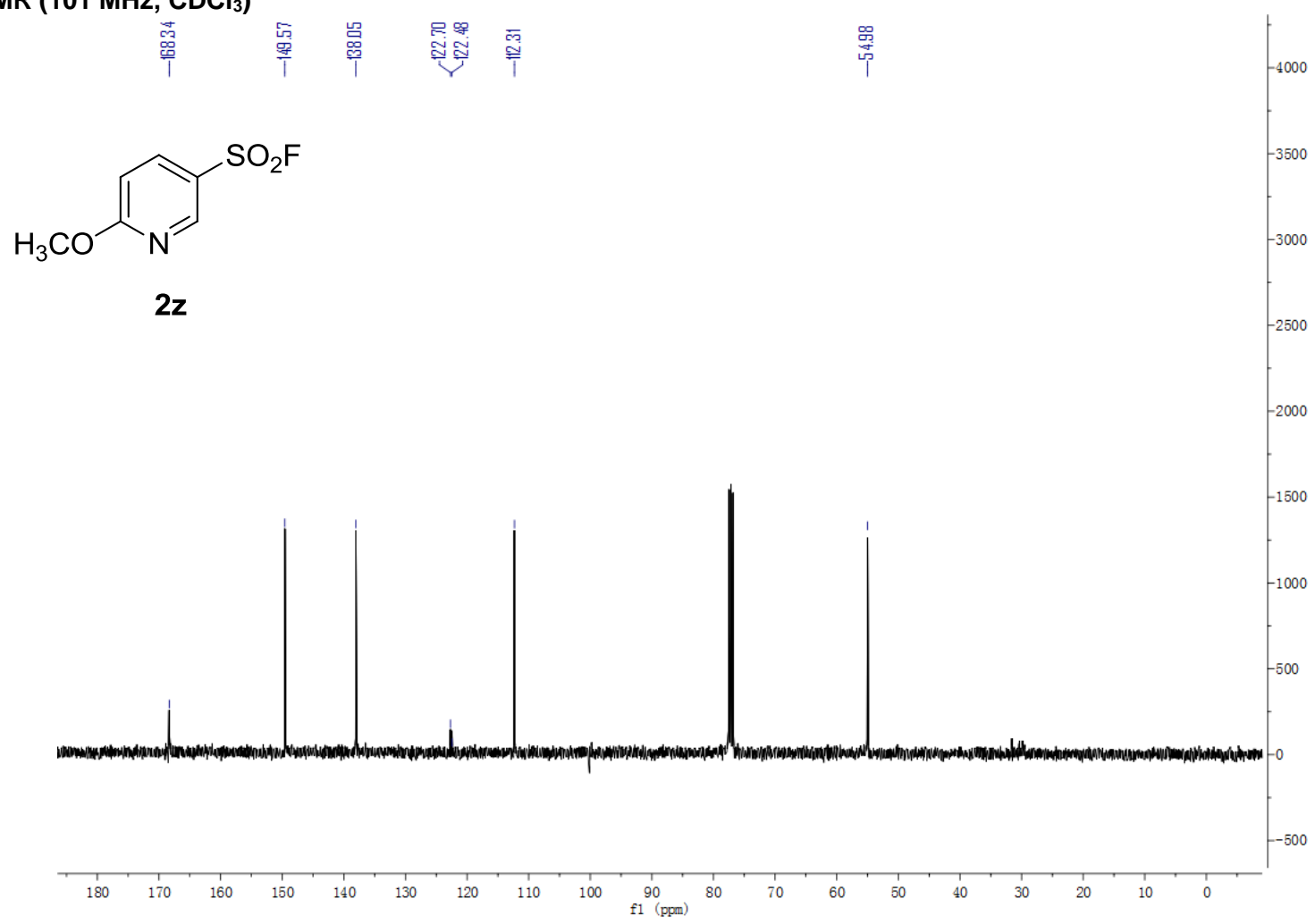

${ }^{19} \mathrm{~F} \mathrm{NMR} \mathrm{(376} \mathrm{MHz,} \mathrm{CDCl}_{3}$ )

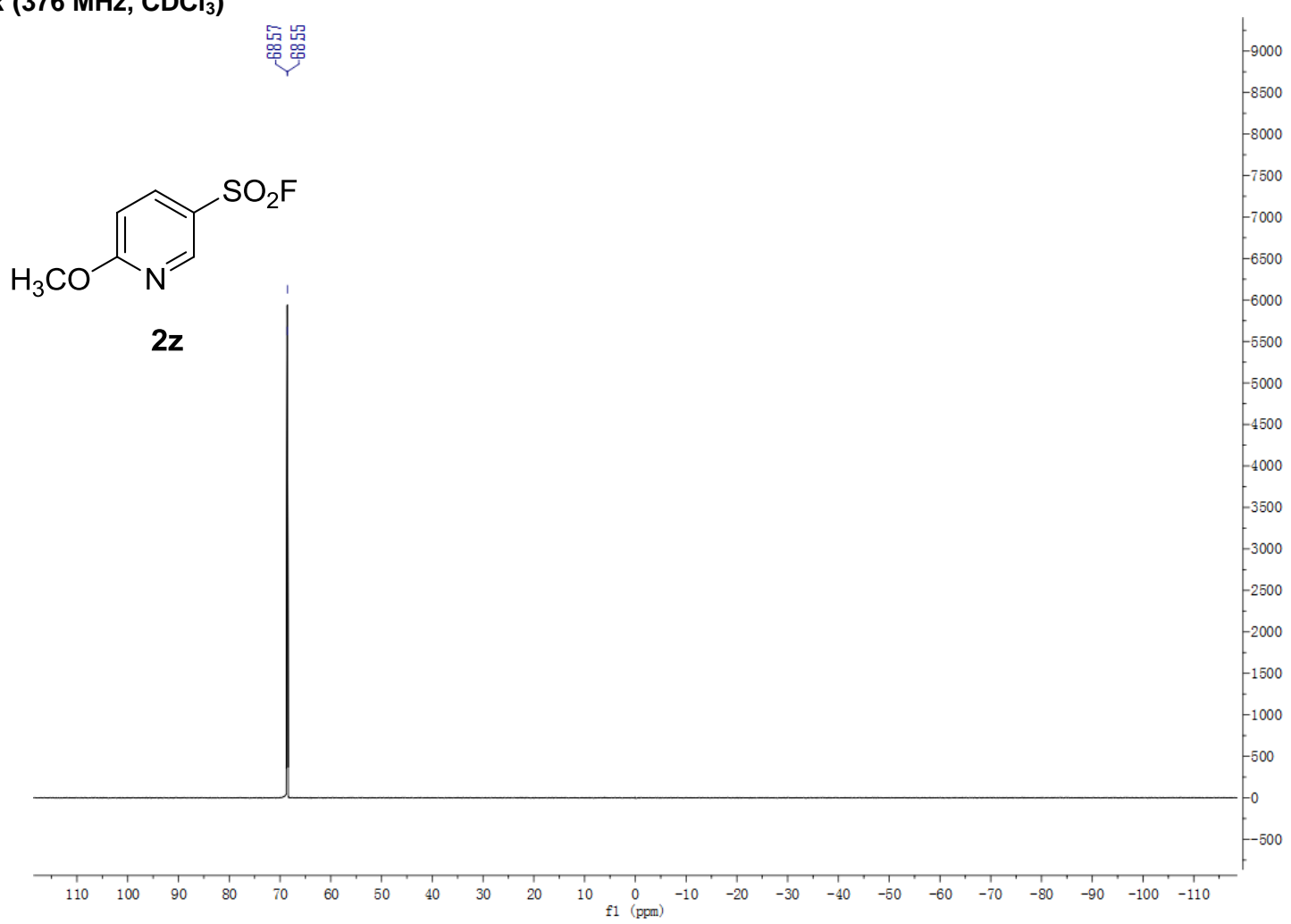


${ }^{1} \mathrm{H}$ NMR (400 MHz, $\mathrm{CDCl}_{3}$ )

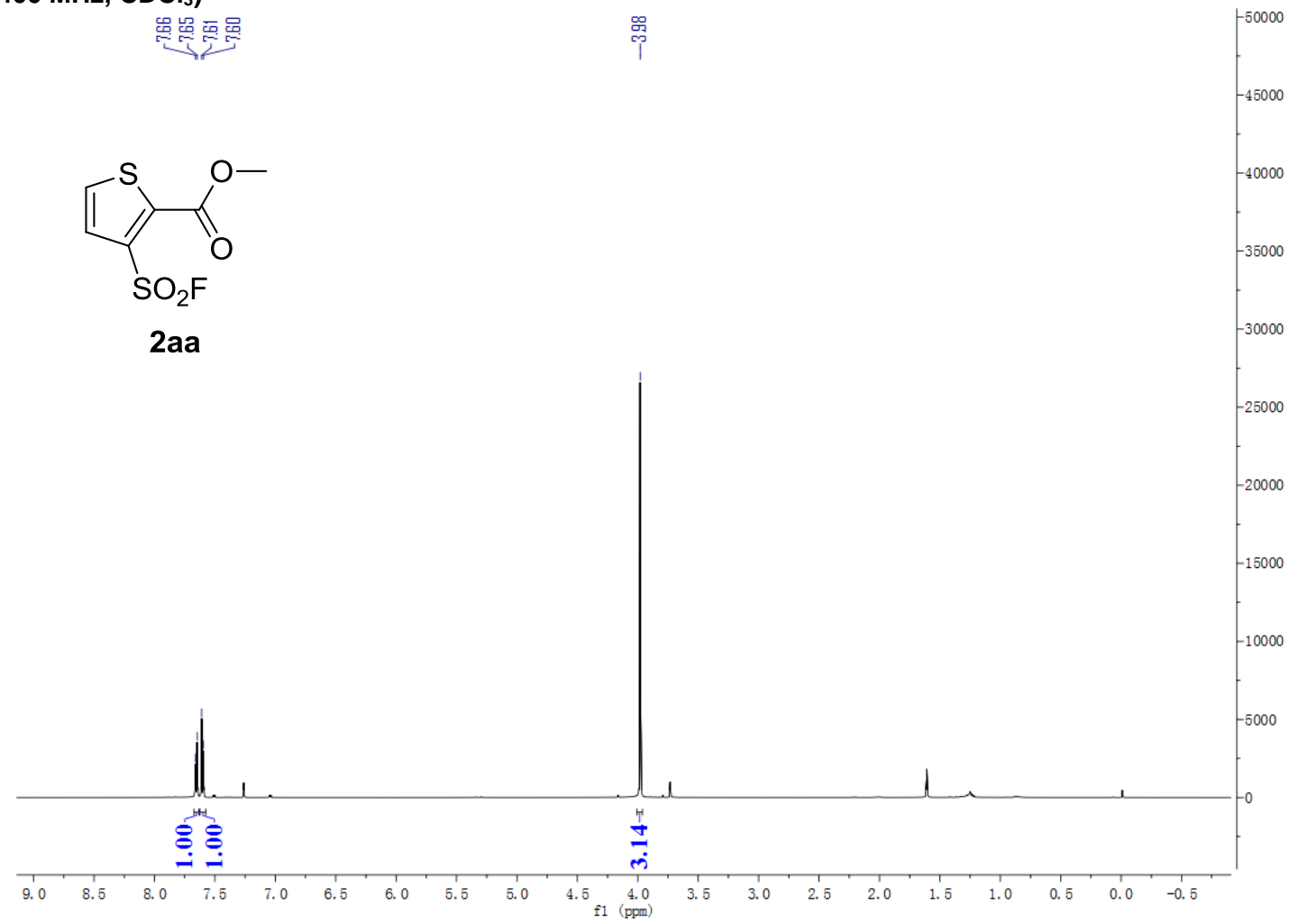

${ }^{13} \mathrm{C}$ NMR (101 MHz, $\mathrm{CDCl}_{3}$ )

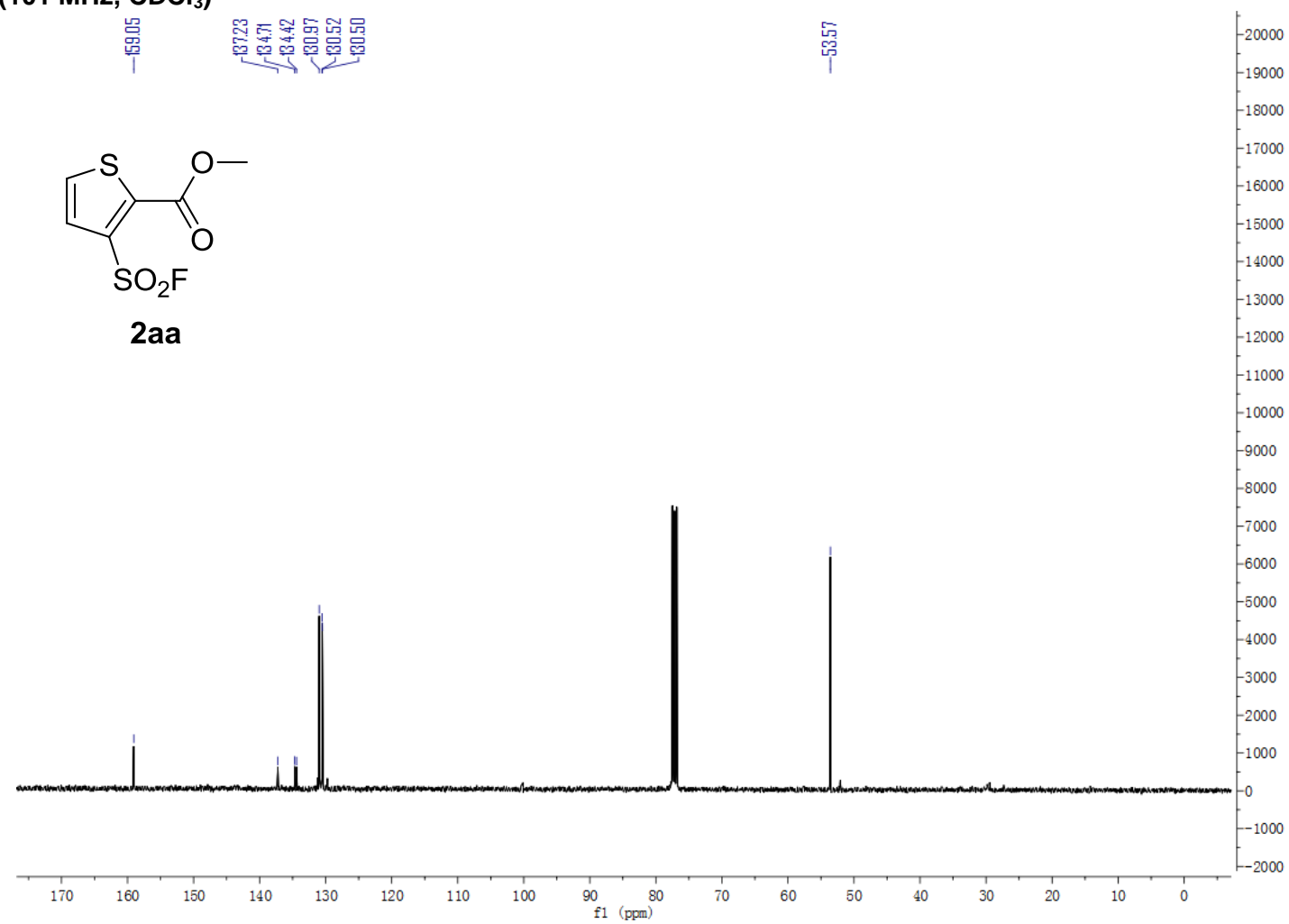


${ }^{19} \mathrm{~F}$ NMR (376 MHz, $\mathrm{CDCl}_{3}$ )

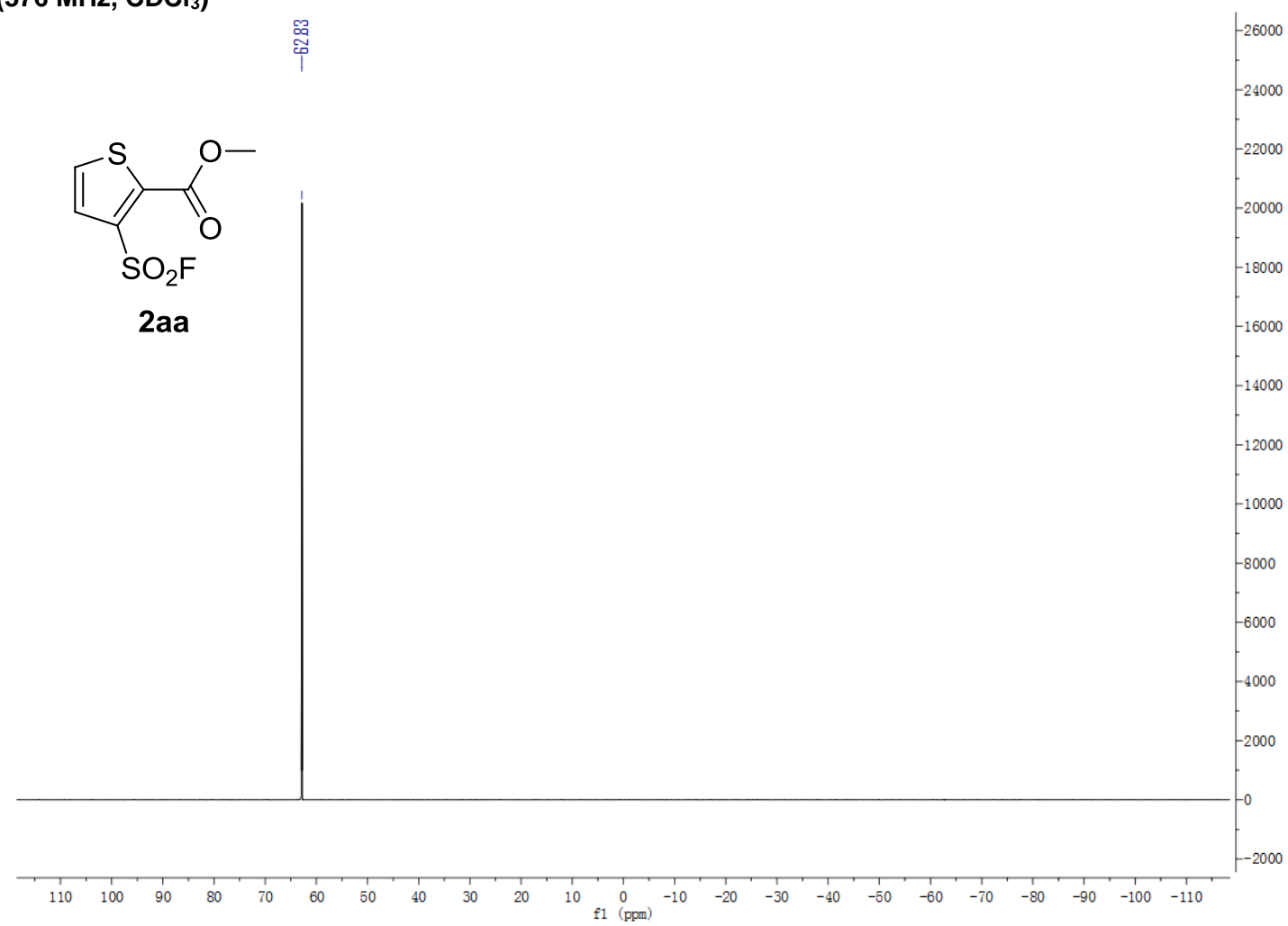

${ }^{1} \mathrm{H}$ NMR $\left(400 \mathrm{MHz}, \mathrm{CDCl}_{3}\right)$

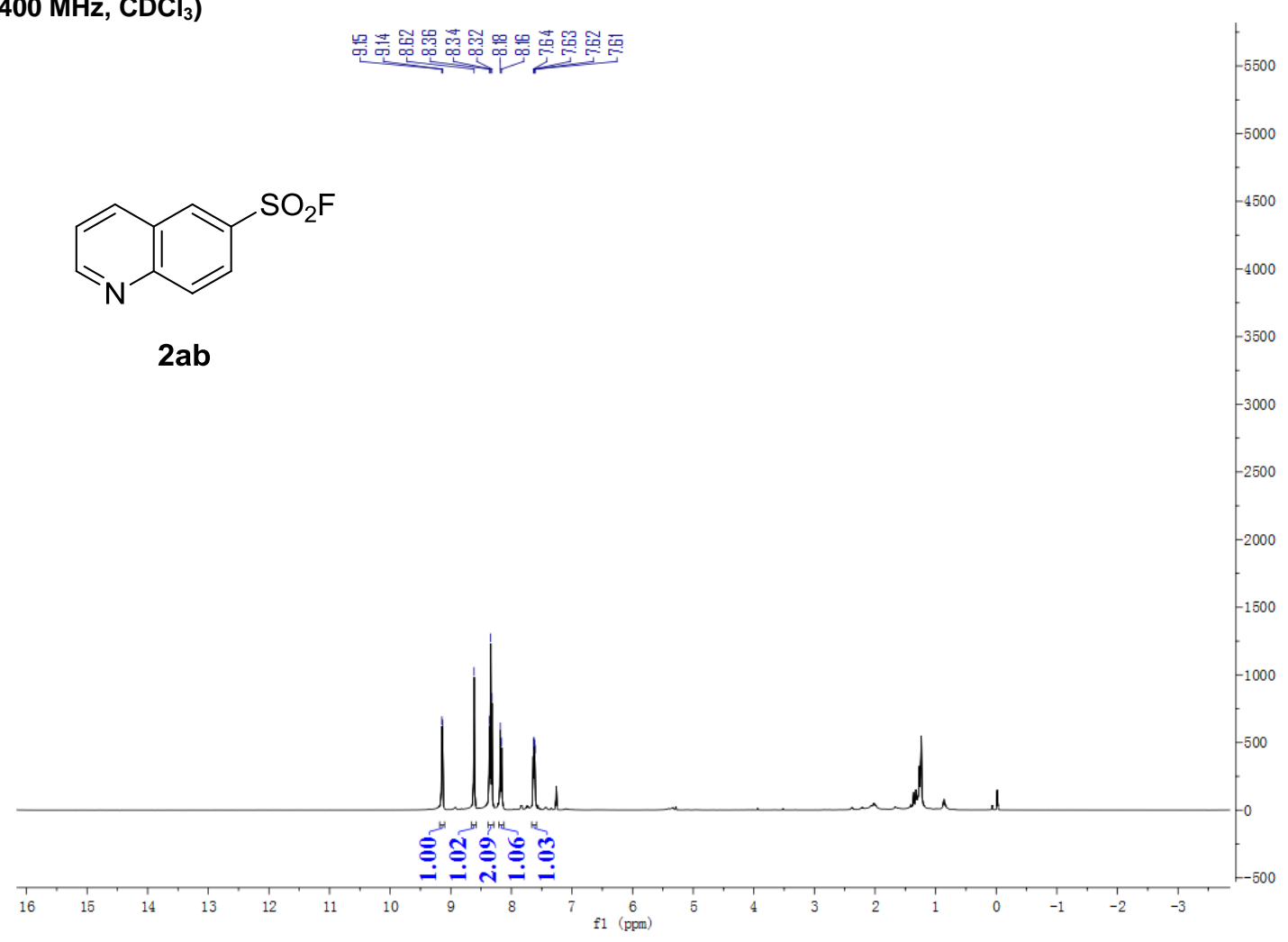


${ }^{13} \mathrm{C}$ NMR (126 MHz, $\mathrm{CDCl}_{3}$ )

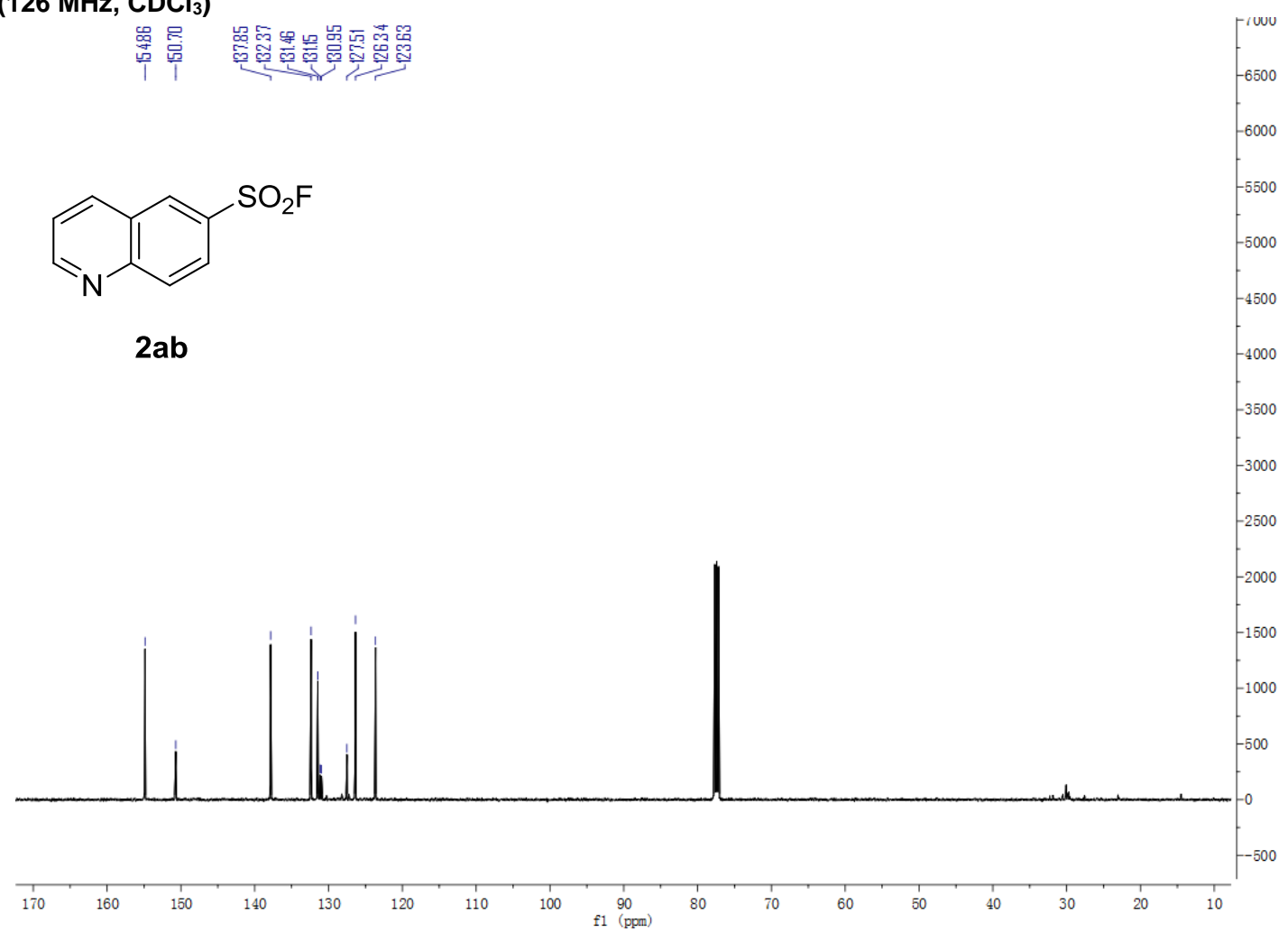

$\left.{ }^{19} \mathrm{~F} \mathrm{NMR} \mathrm{(376} \mathrm{MHz,} \mathrm{CDCl}_{3}\right)$

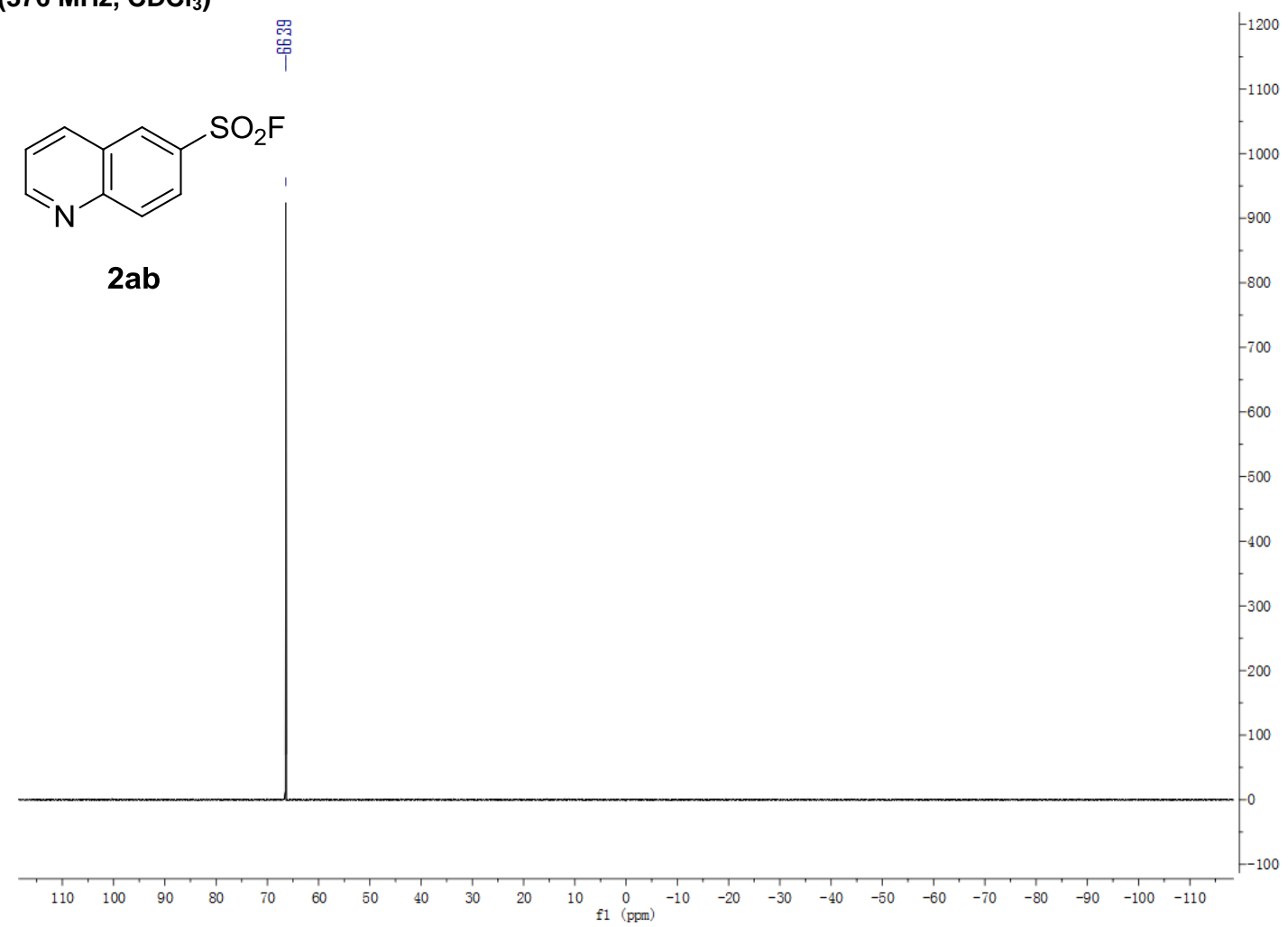


${ }^{1} \mathrm{H}$ NMR (400 MHz, $\mathrm{CDCl}_{3}$ )

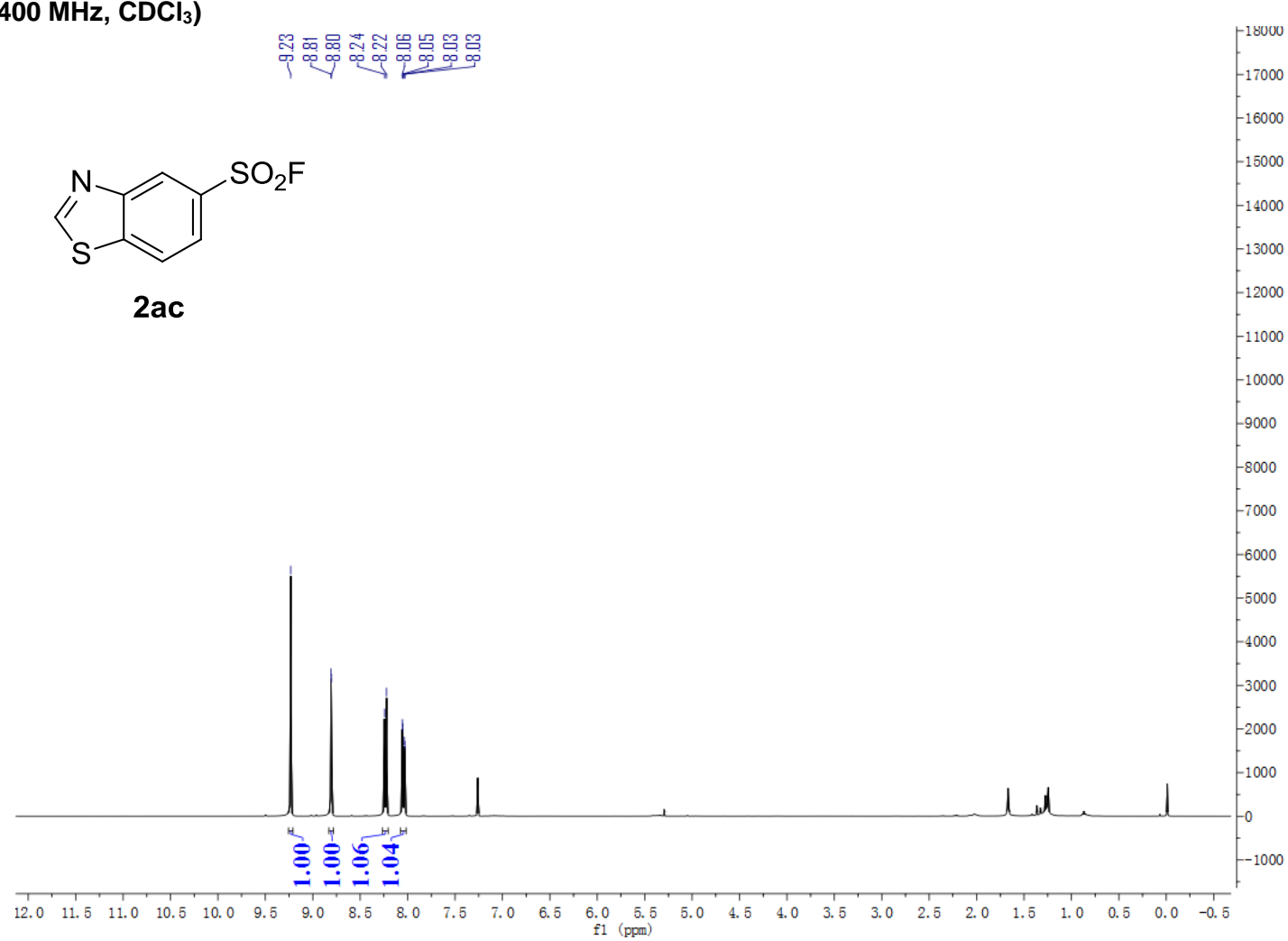

${ }^{13} \mathrm{C}$ NMR (101 MHz, $\mathrm{CDCl}_{3}$ )

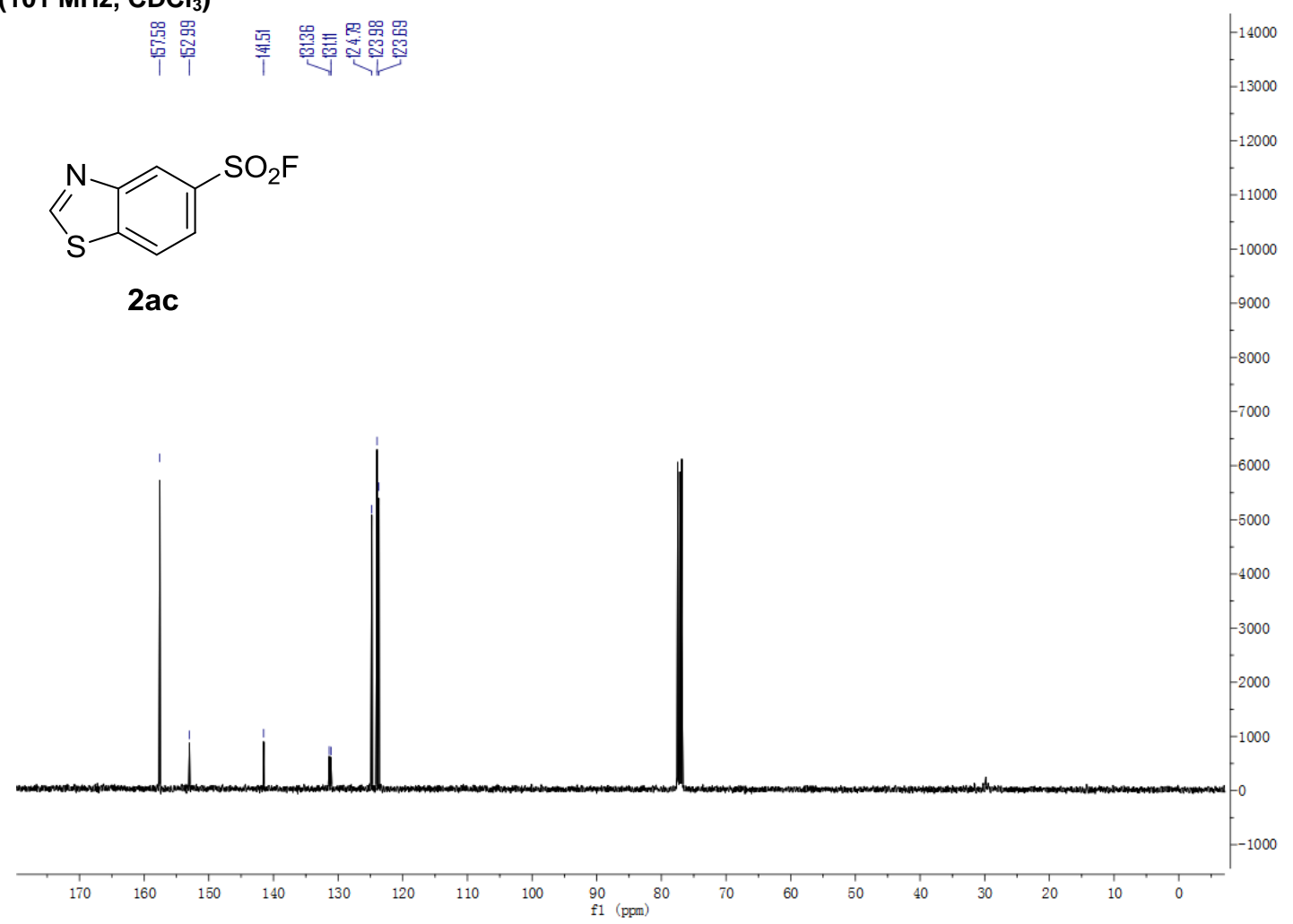


${ }^{19} \mathrm{~F}$ NMR (376 MHz, $\mathrm{CDCl}_{3}$ )

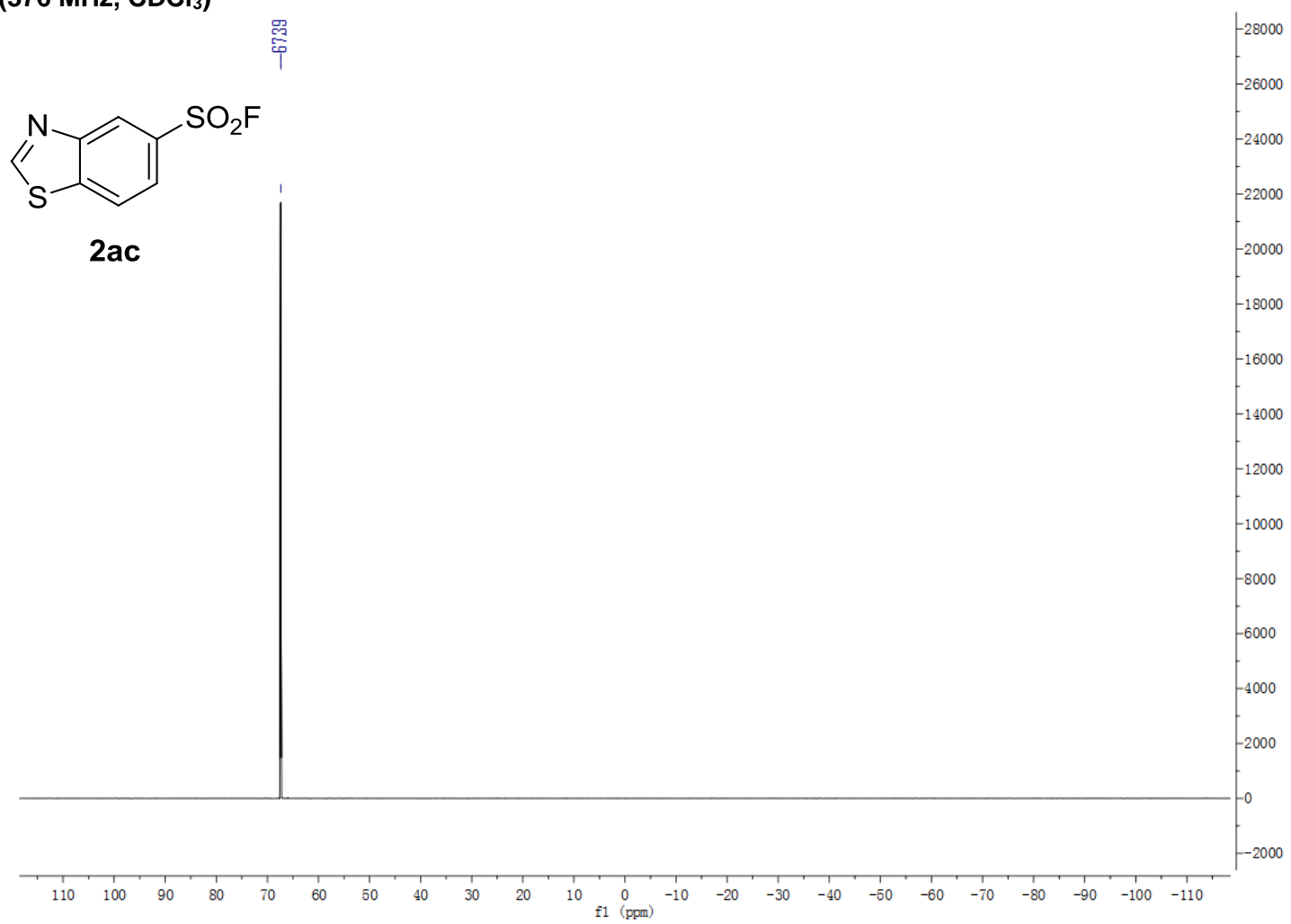

${ }^{1} \mathrm{H}$ NMR (400 MHz, $\mathrm{CDCl}_{3}$ )

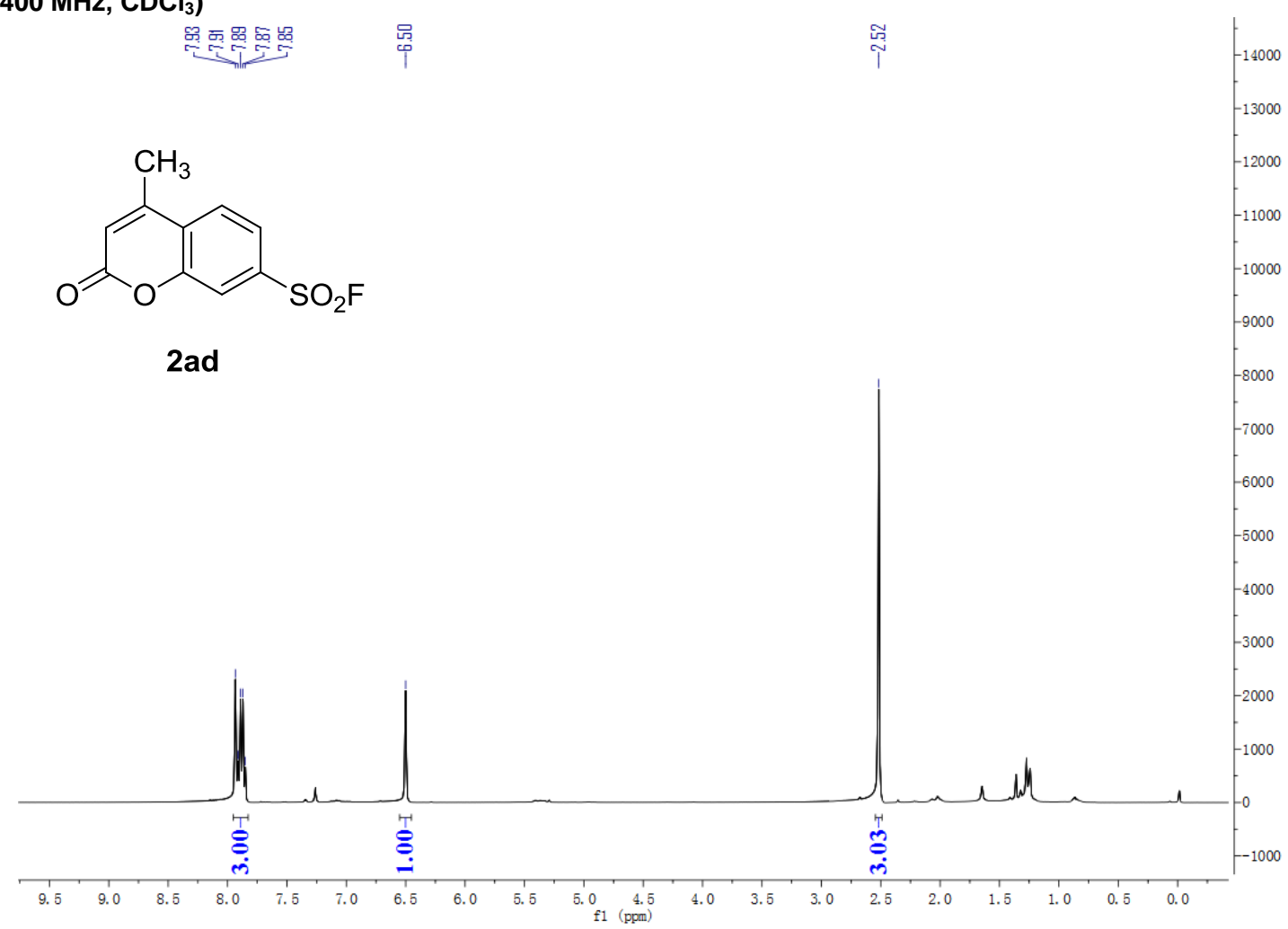


${ }^{13} \mathrm{C}$ NMR (101 MHz, CDCl $)$

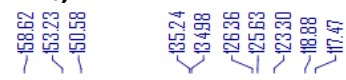<smiles>Cc1cc(=O)oc2cc(S(=O)(=O)OF)ccc12</smiles>

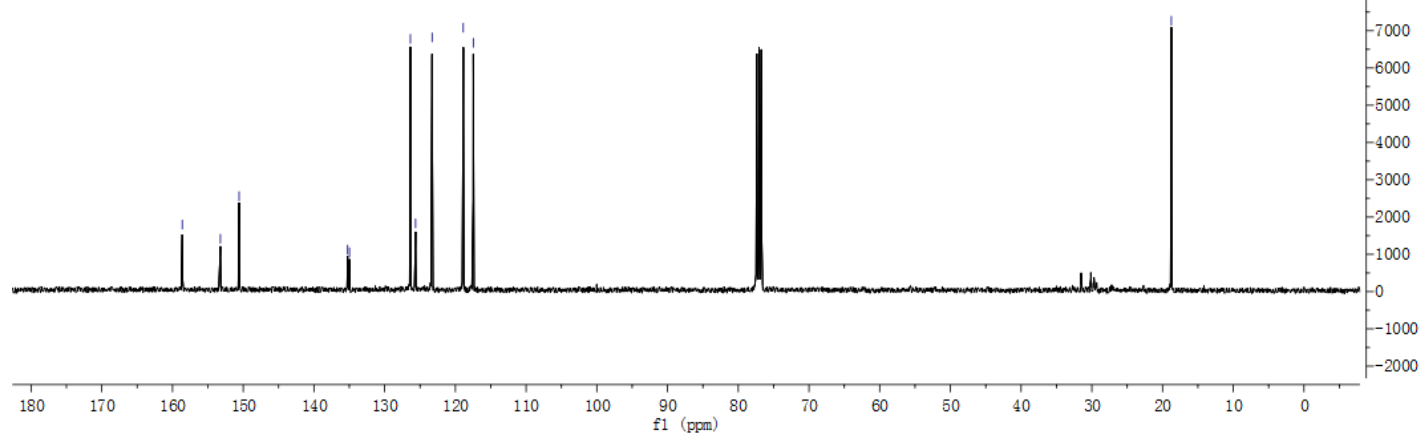

${ }^{19} \mathrm{~F}$ NMR (376 MHz, $\mathrm{CDCl}_{3}$ )

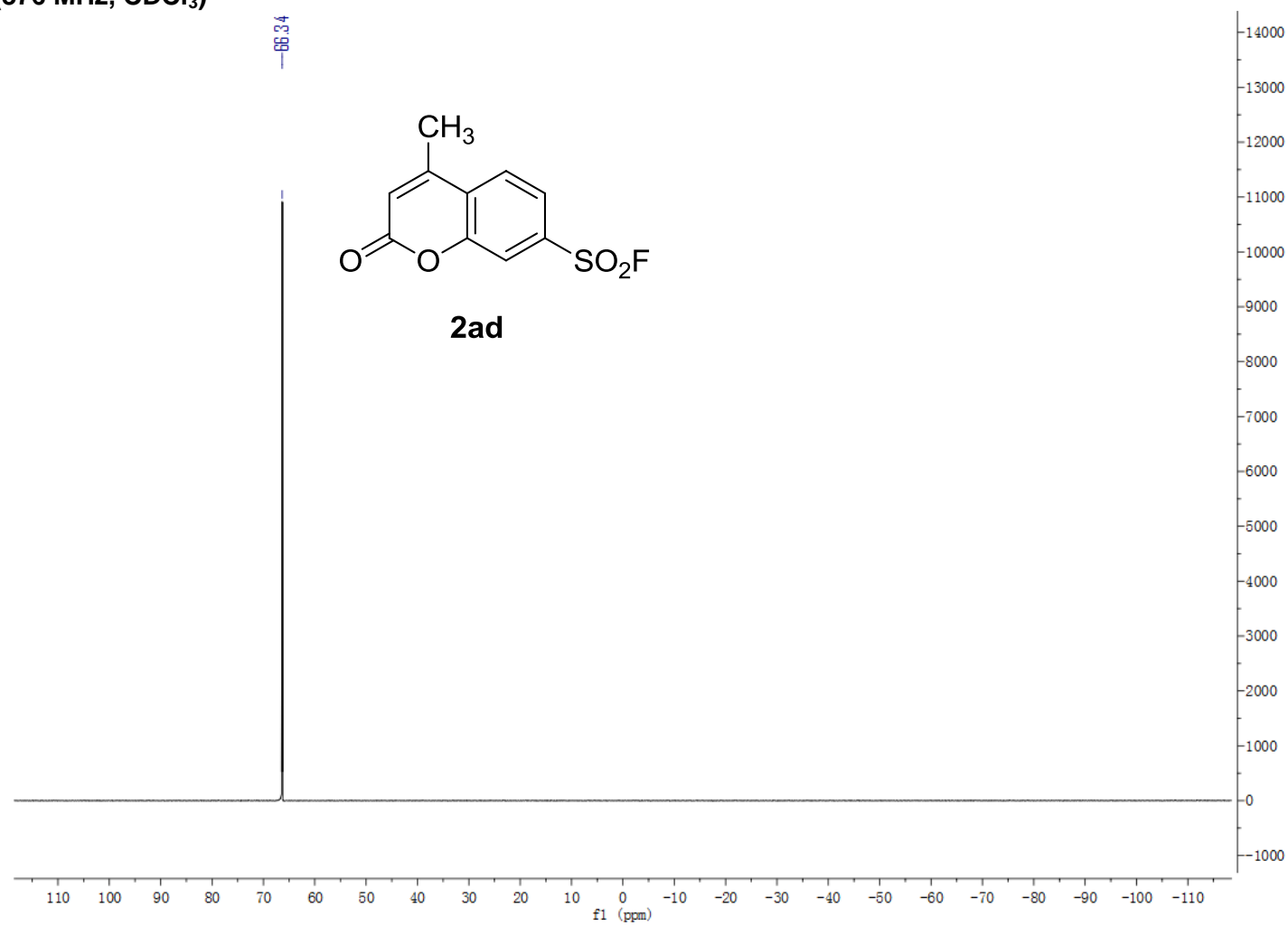


${ }^{1} \mathrm{H} \mathrm{NMR}\left(400 \mathrm{MHz}, \mathrm{CDCl}_{3}\right)$

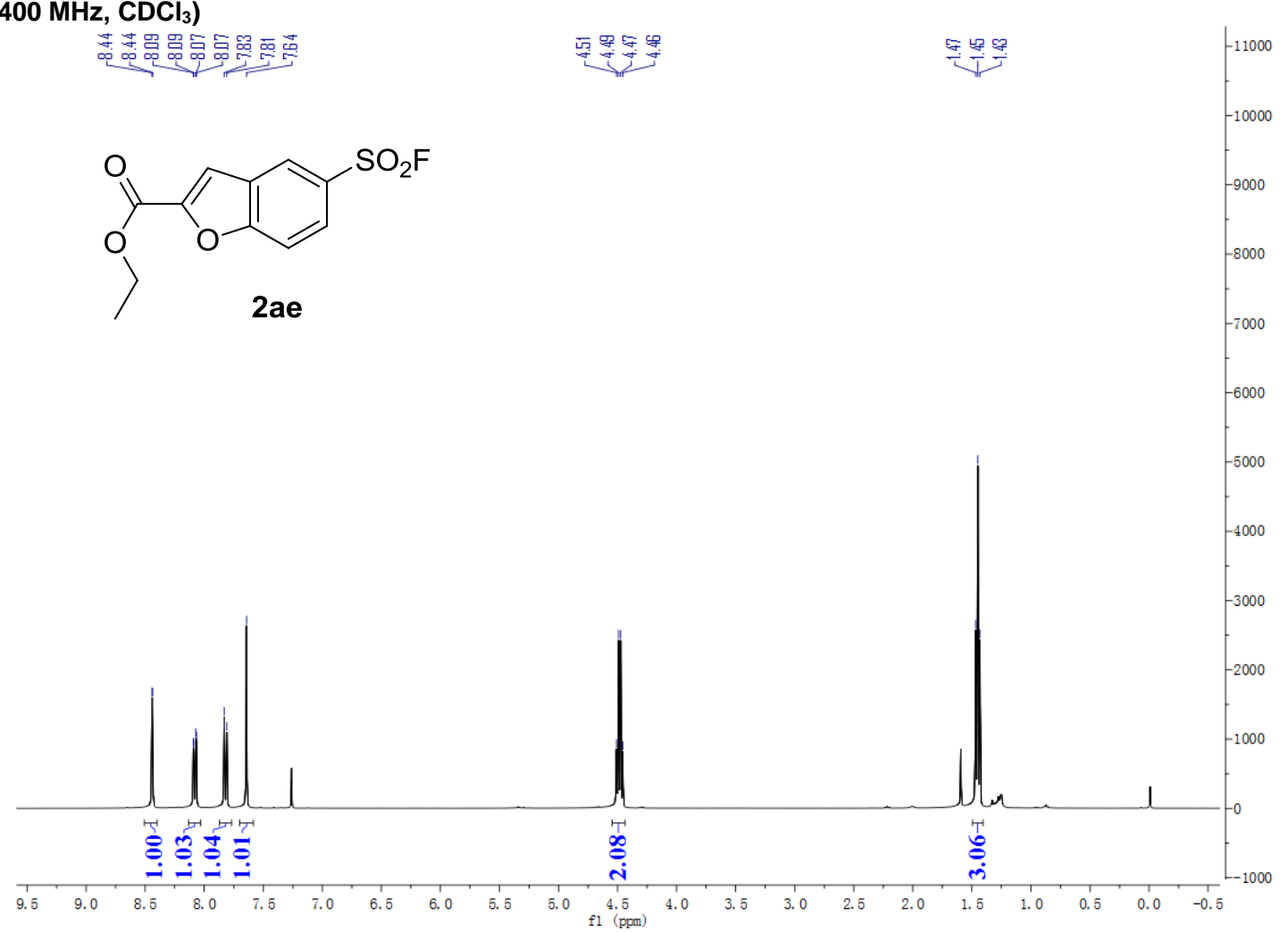

${ }^{13} \mathrm{C}$ NMR (101 MHz, $\mathrm{CDCl}_{3}$ )

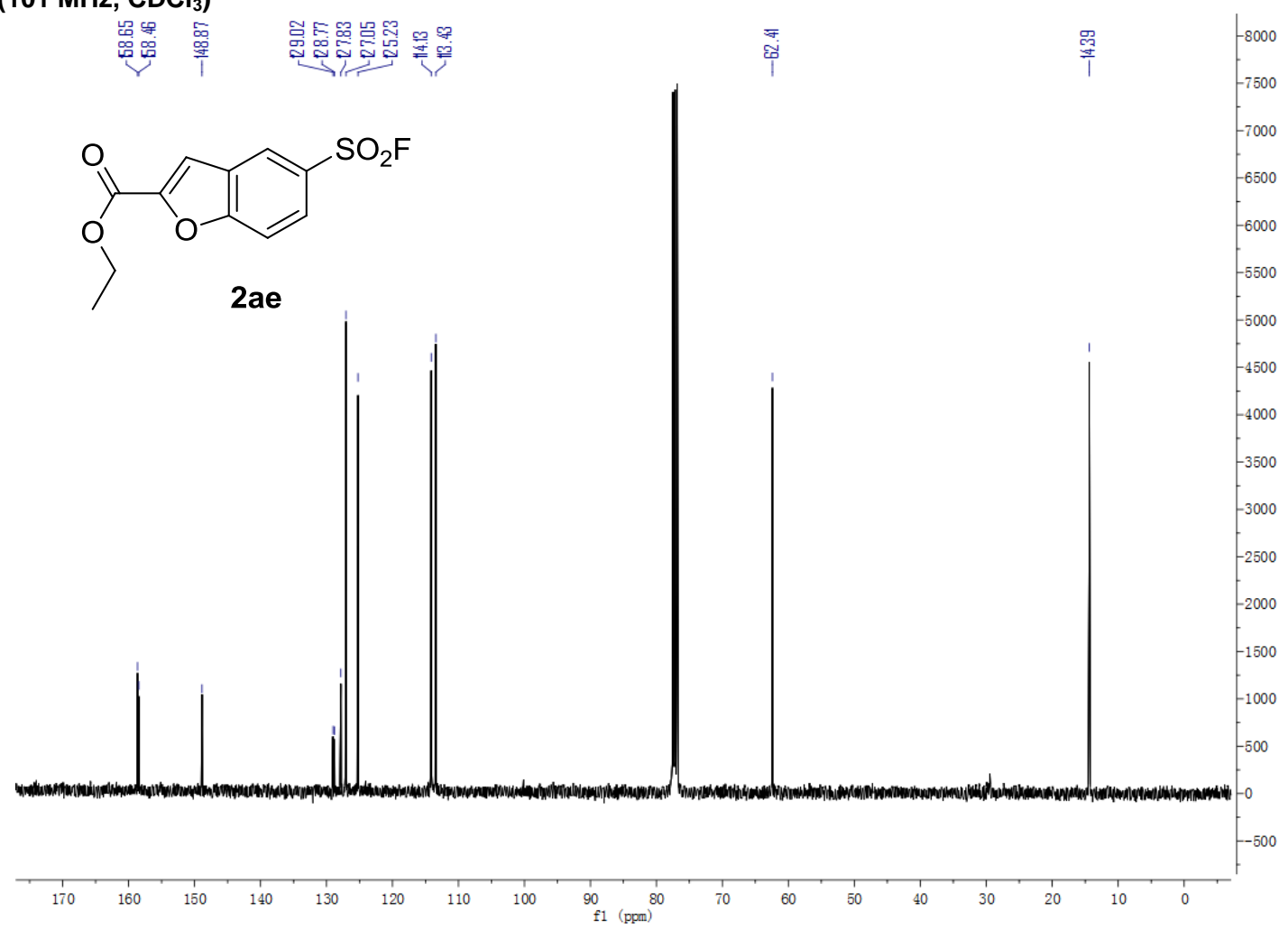


${ }^{19} \mathrm{~F} \mathrm{NMR} \mathrm{(376} \mathrm{MHz,} \mathrm{CDCl}_{3}$ )

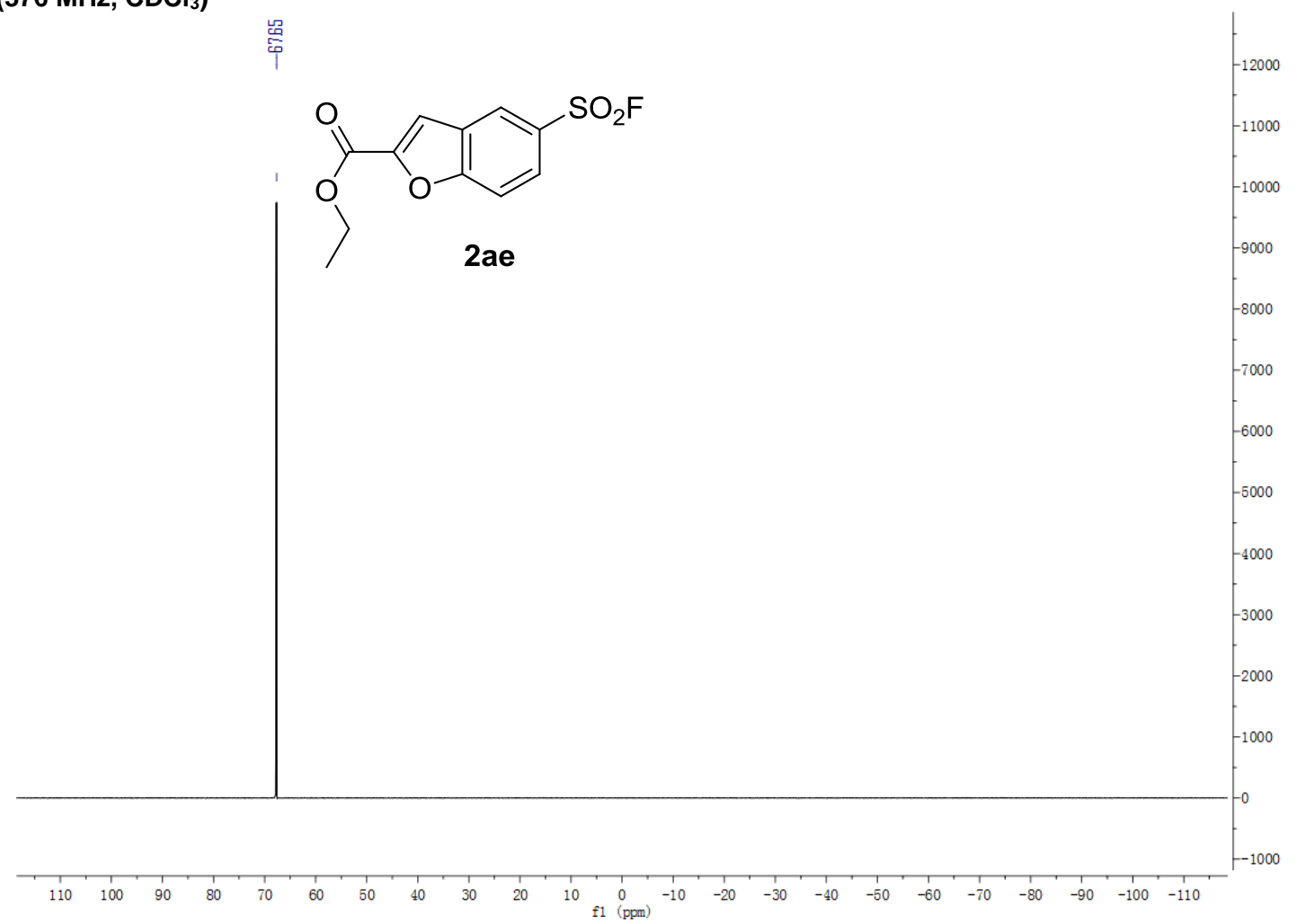

${ }^{1} \mathrm{H}$ NMR (400 MHz, DMSO-d ${ }_{6}$ )

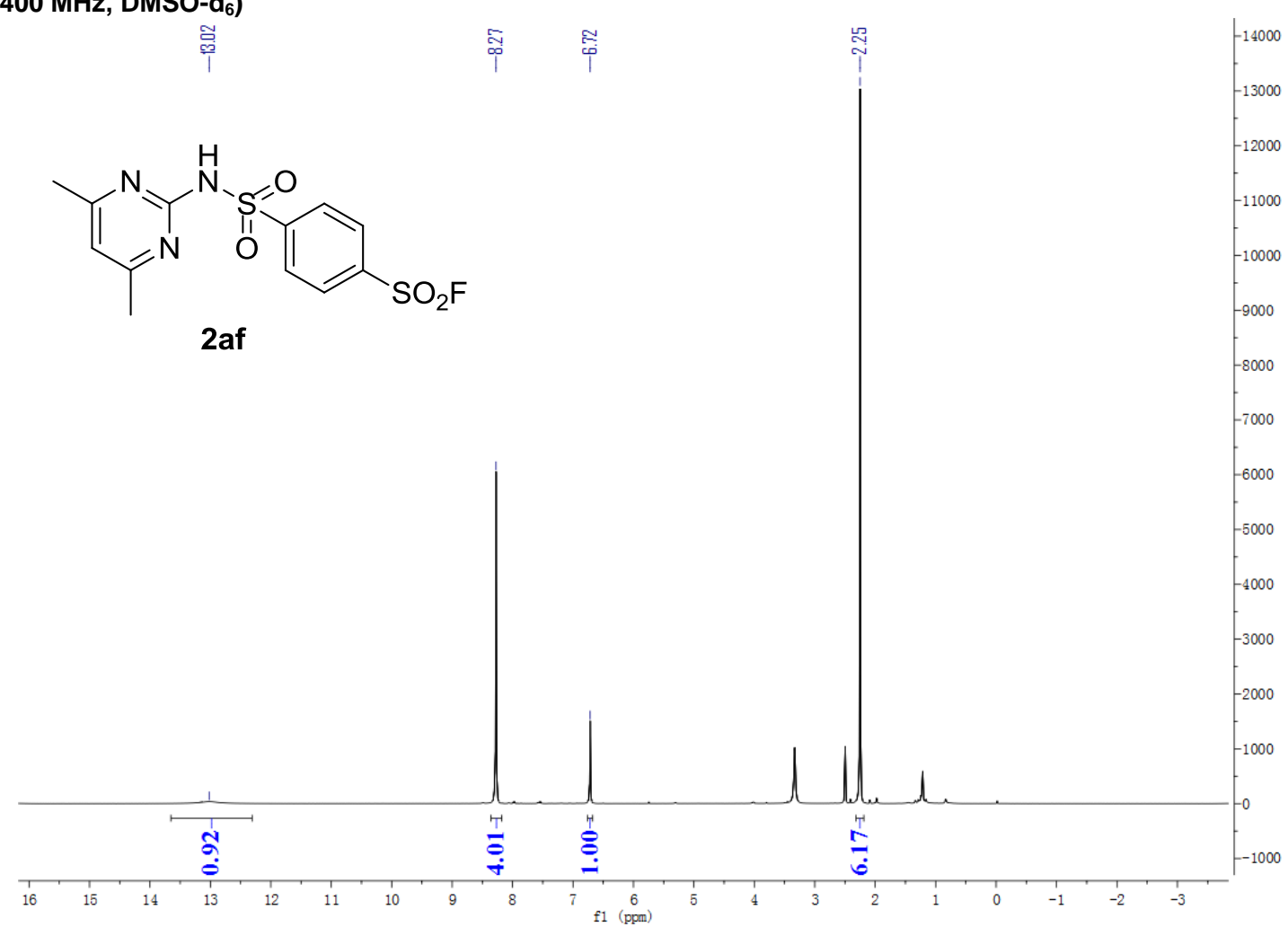


${ }^{13} \mathrm{C}$ NMR (126 MHz, DMSO-d 6 )

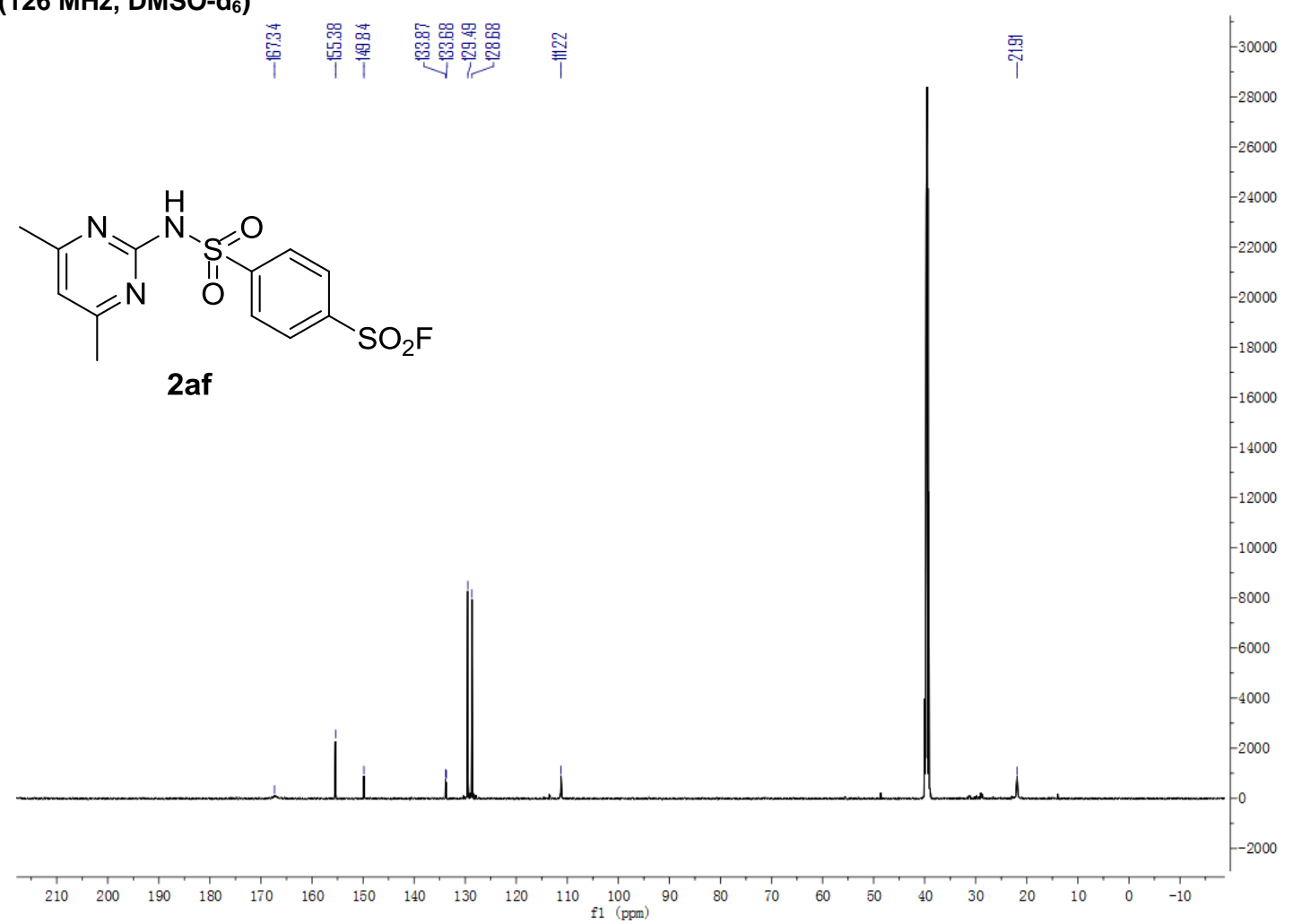

${ }^{19}$ F NMR (376 MHz, DMSO-d )

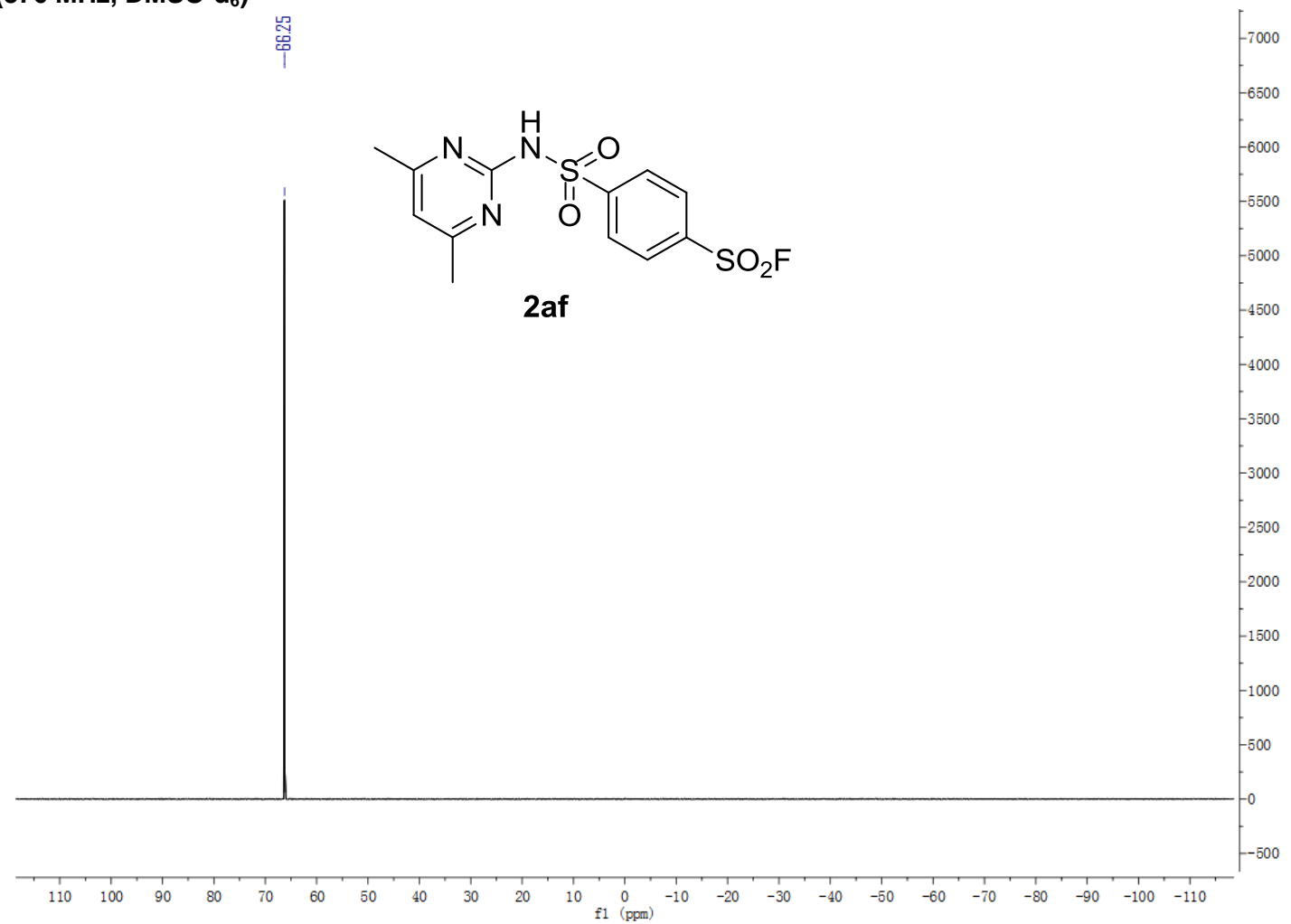




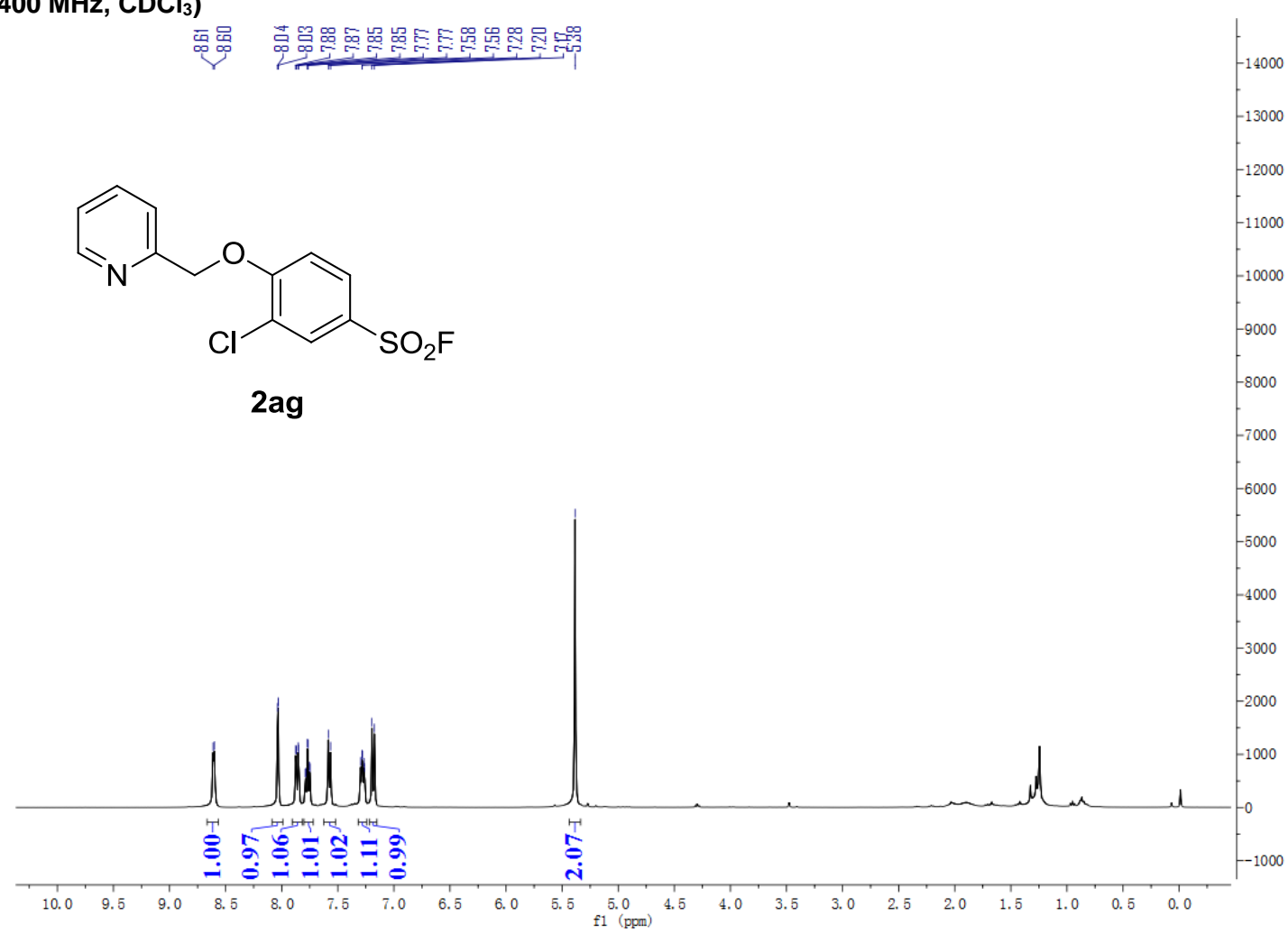

${ }^{13} \mathrm{C}$ NMR (101 MHz, CDCl $)$

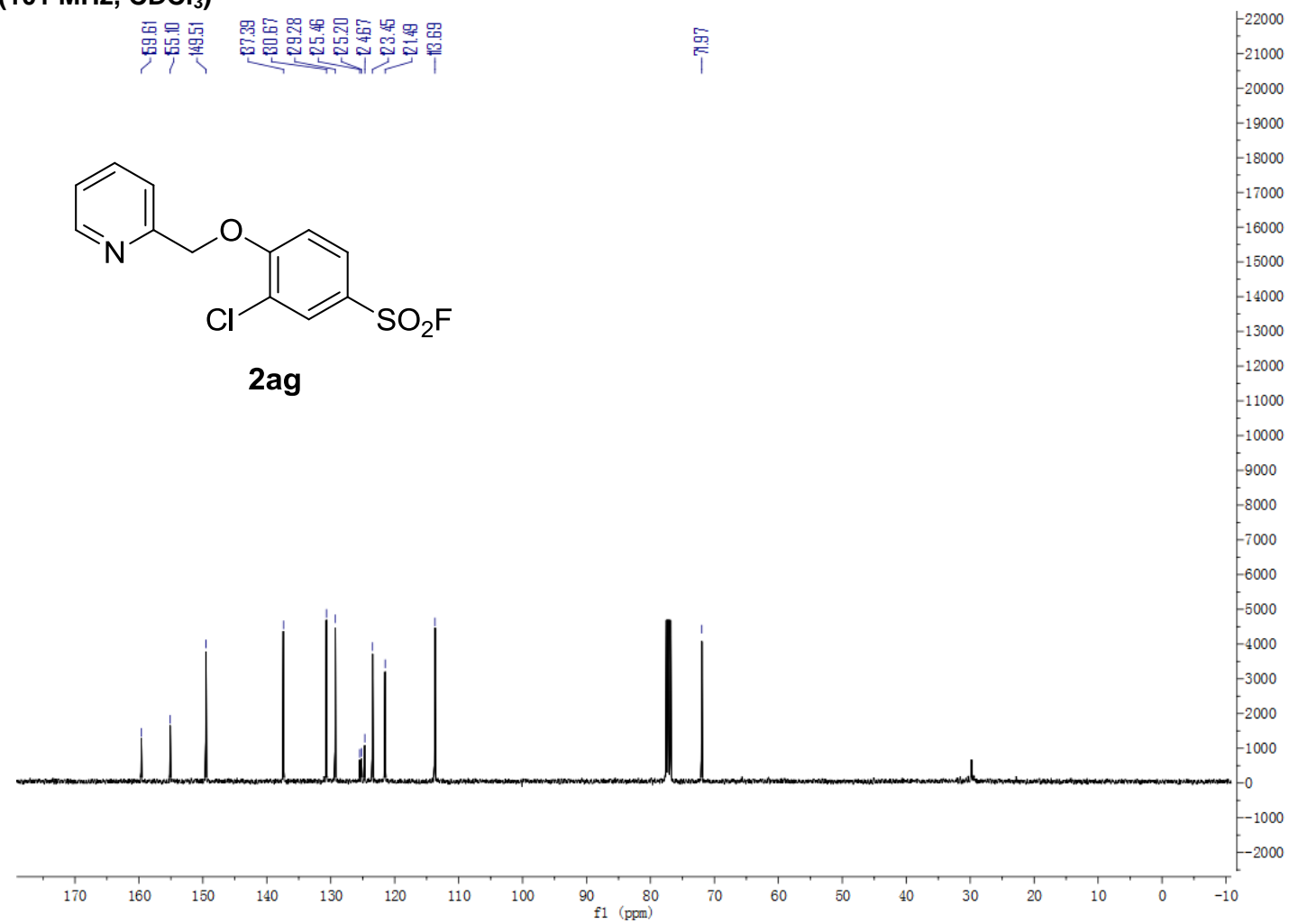


${ }^{19} \mathrm{~F}$ NMR (376 MHz, $\mathrm{CDCl}_{3}$ )

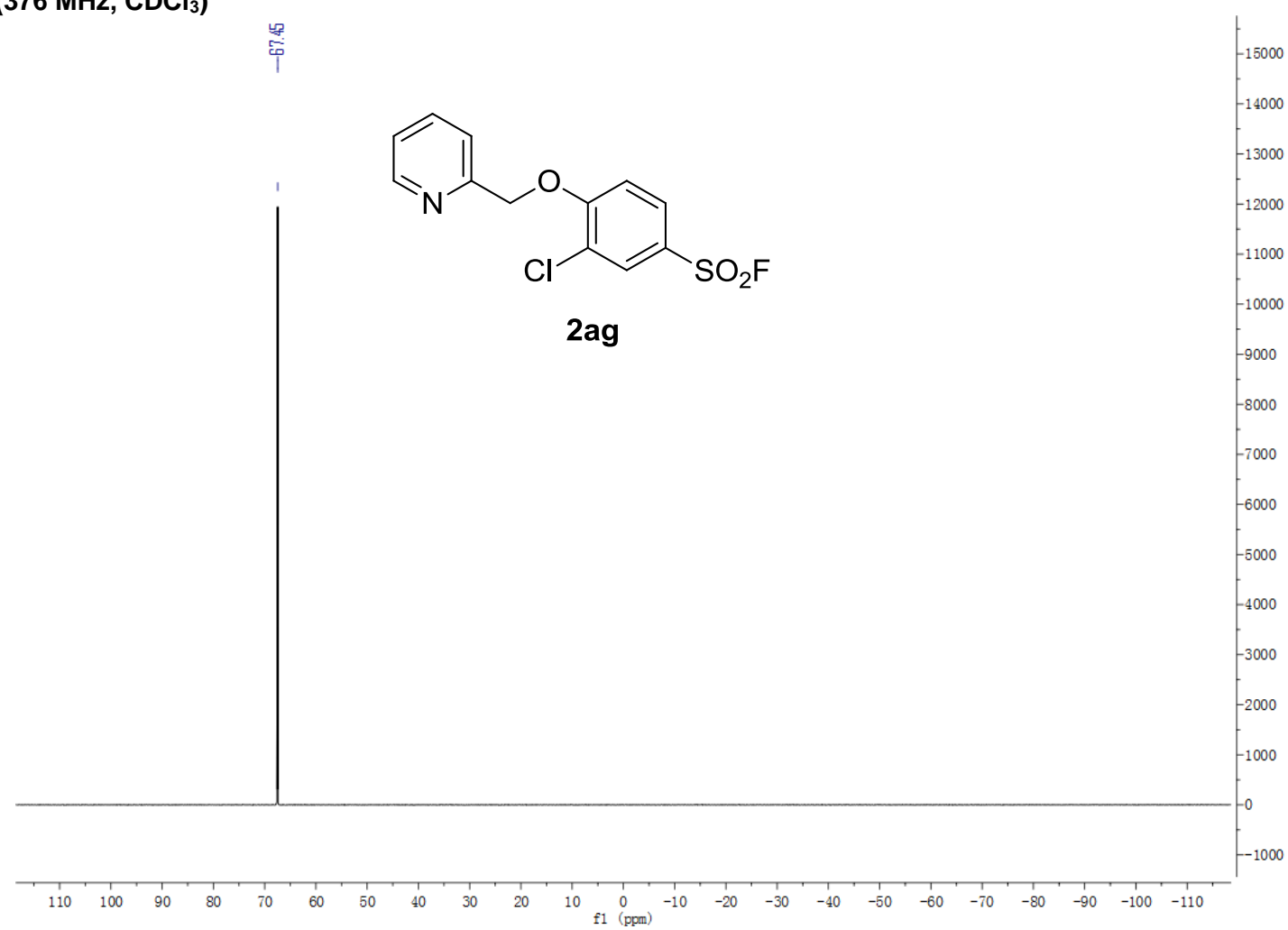

${ }^{1} \mathrm{H}$ NMR $\left(400 \mathrm{MHz}, \mathrm{CDCl}_{3}\right)$

血哭品器

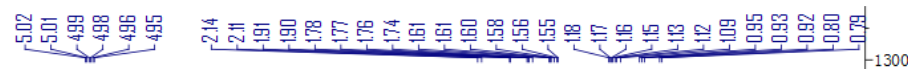<smiles>COS(=O)(=O)c1ccc(C(=O)O[C@@H]2C[C@H](C)CCC2C(C)C)cc1</smiles>

2ah

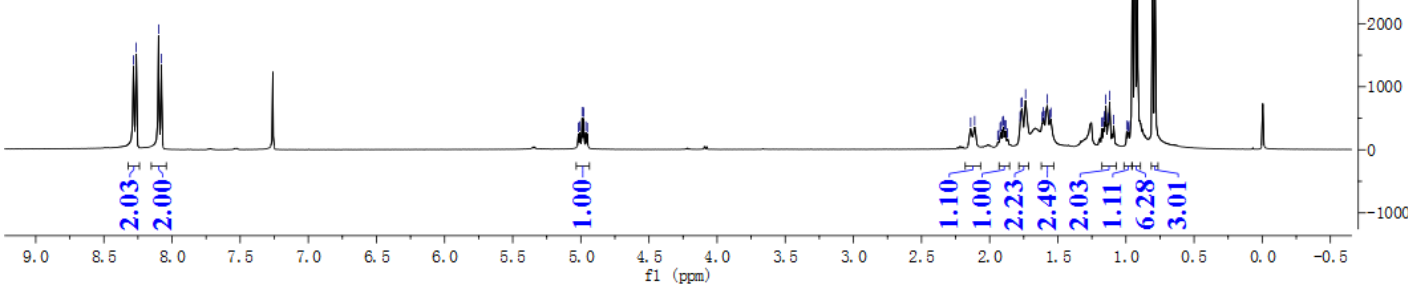


${ }^{13} \mathrm{C} \mathrm{NMR} \mathrm{(101} \mathrm{MHz,} \mathrm{CDCl}_{3}$ )

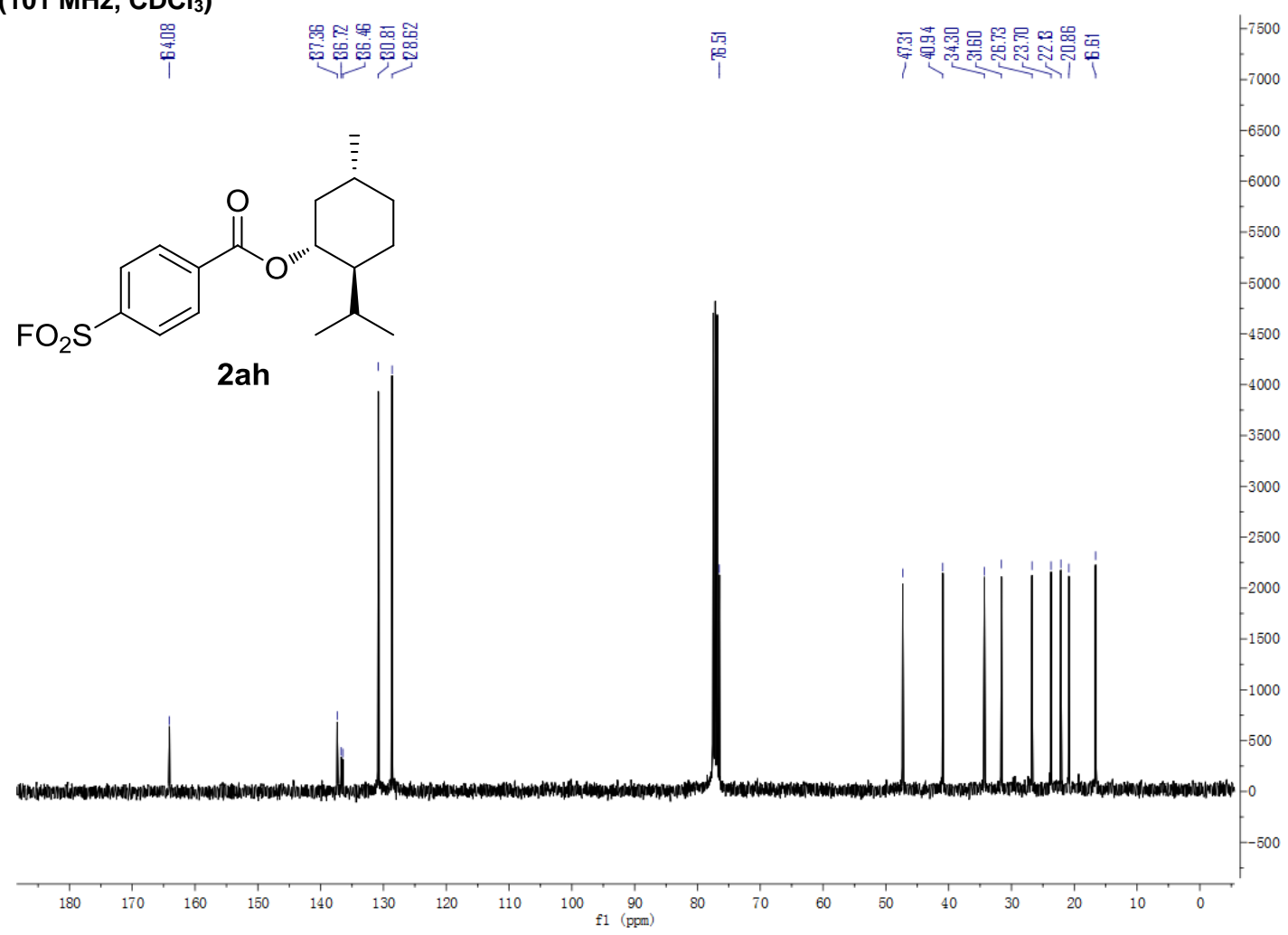

${ }^{19} \mathrm{~F}$ NMR (376 MHz, $\mathrm{CDCl}_{3}$ )

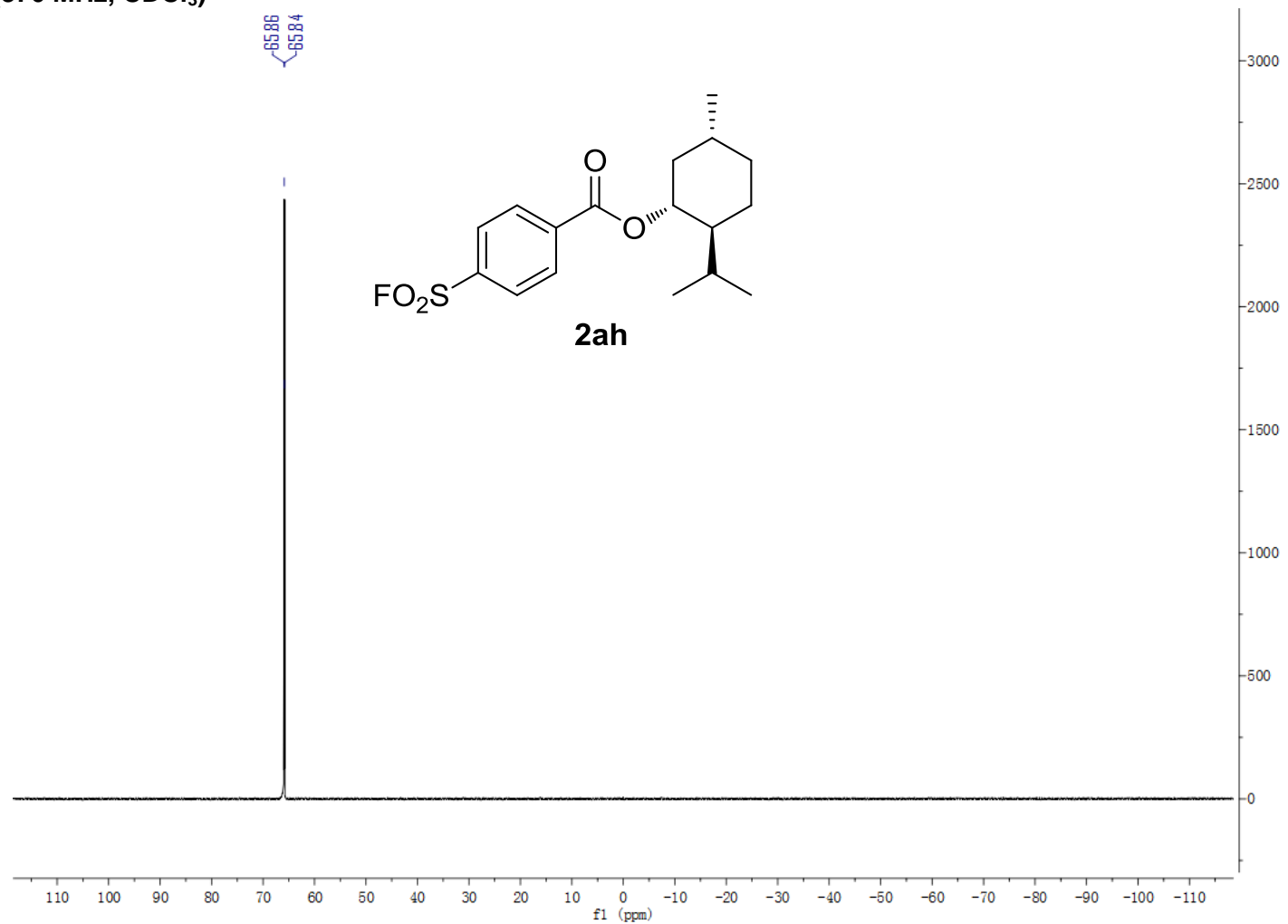


${ }^{1} \mathrm{H}$ NMR $\left(400 \mathrm{MHz}, \mathrm{CDCl}_{3}\right)$

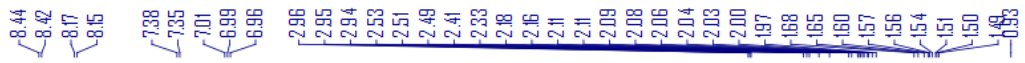<smiles>CC12CCC3c4ccc(OC(=O)c5ccc(S(=O)(=O)O)cc5)cc4CC[C@H]3[C@@H]1CCC2=O</smiles>

2ai

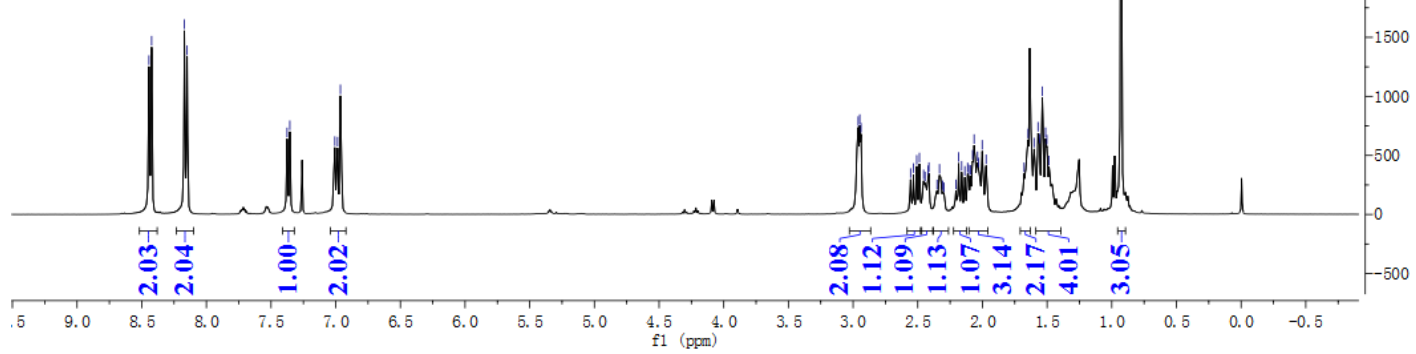

${ }^{13} \mathrm{C}$ NMR (101 MHz, $\mathrm{CDCl}_{3}$ )

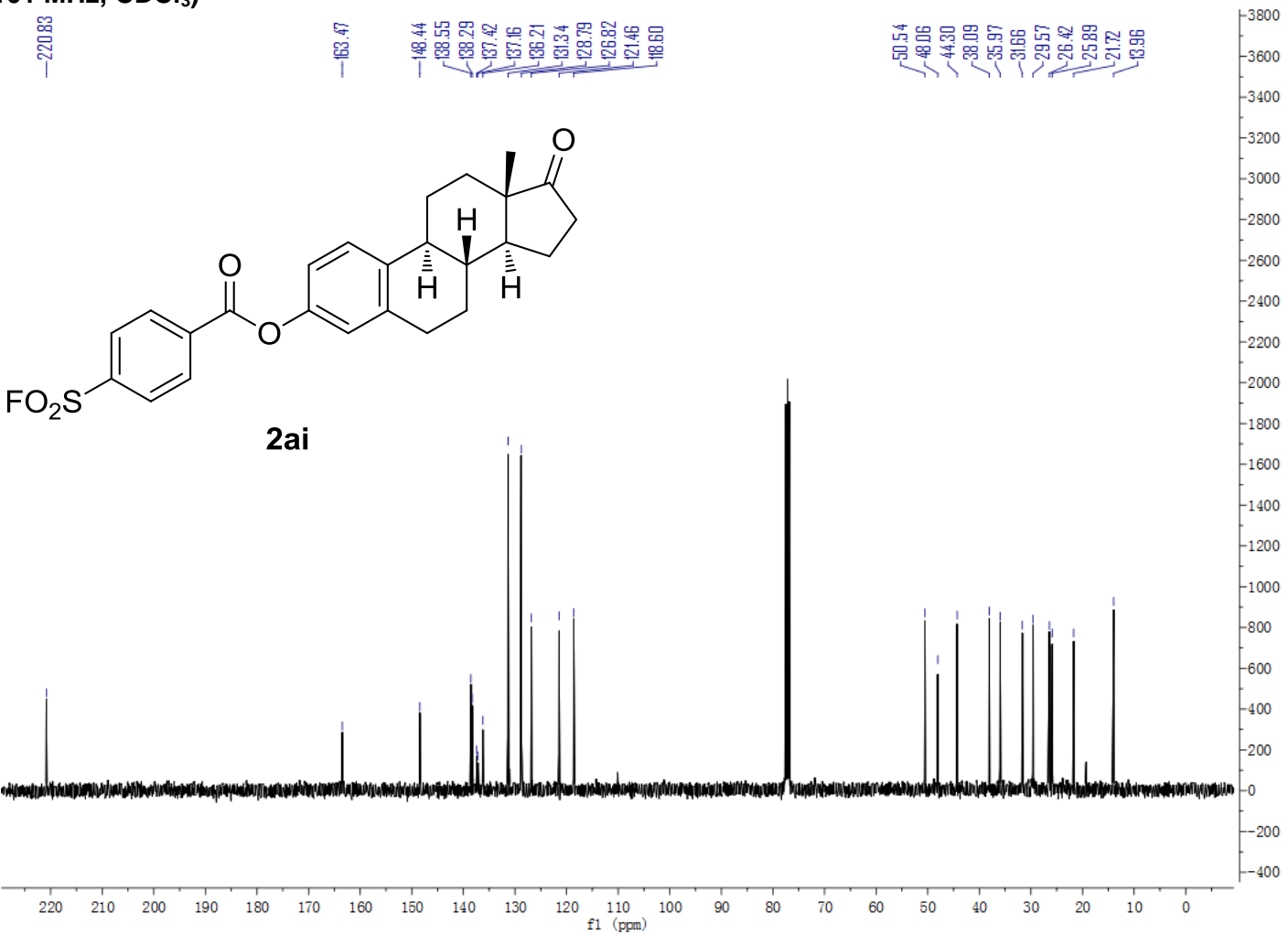


${ }^{19} \mathrm{~F}$ NMR (376 MHz, $\mathrm{CDCl}_{3}$ )

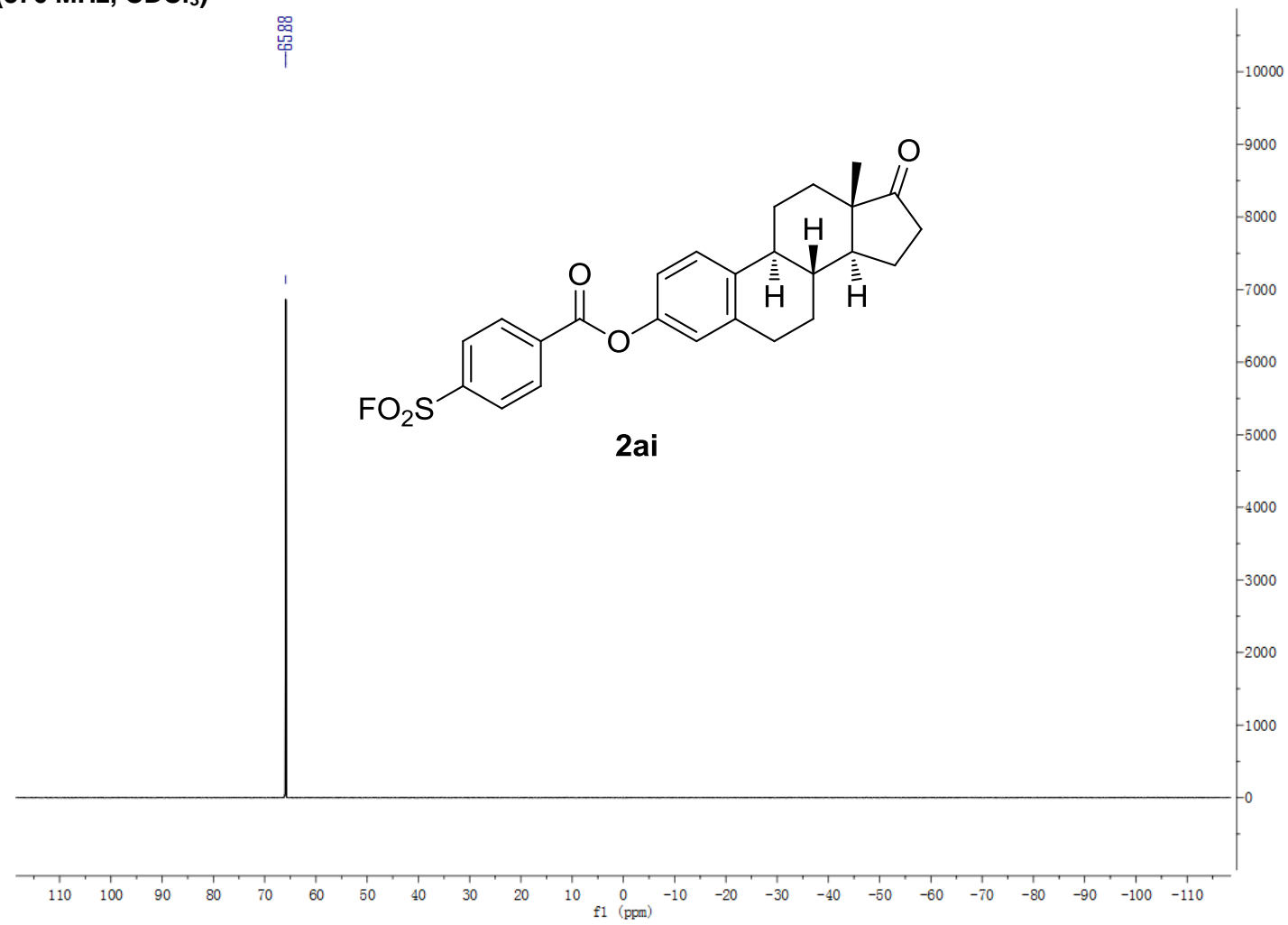

${ }^{1} \mathrm{H}$ NMR $\left(400 \mathrm{MHz}, \mathrm{CDCl}_{3}\right)$

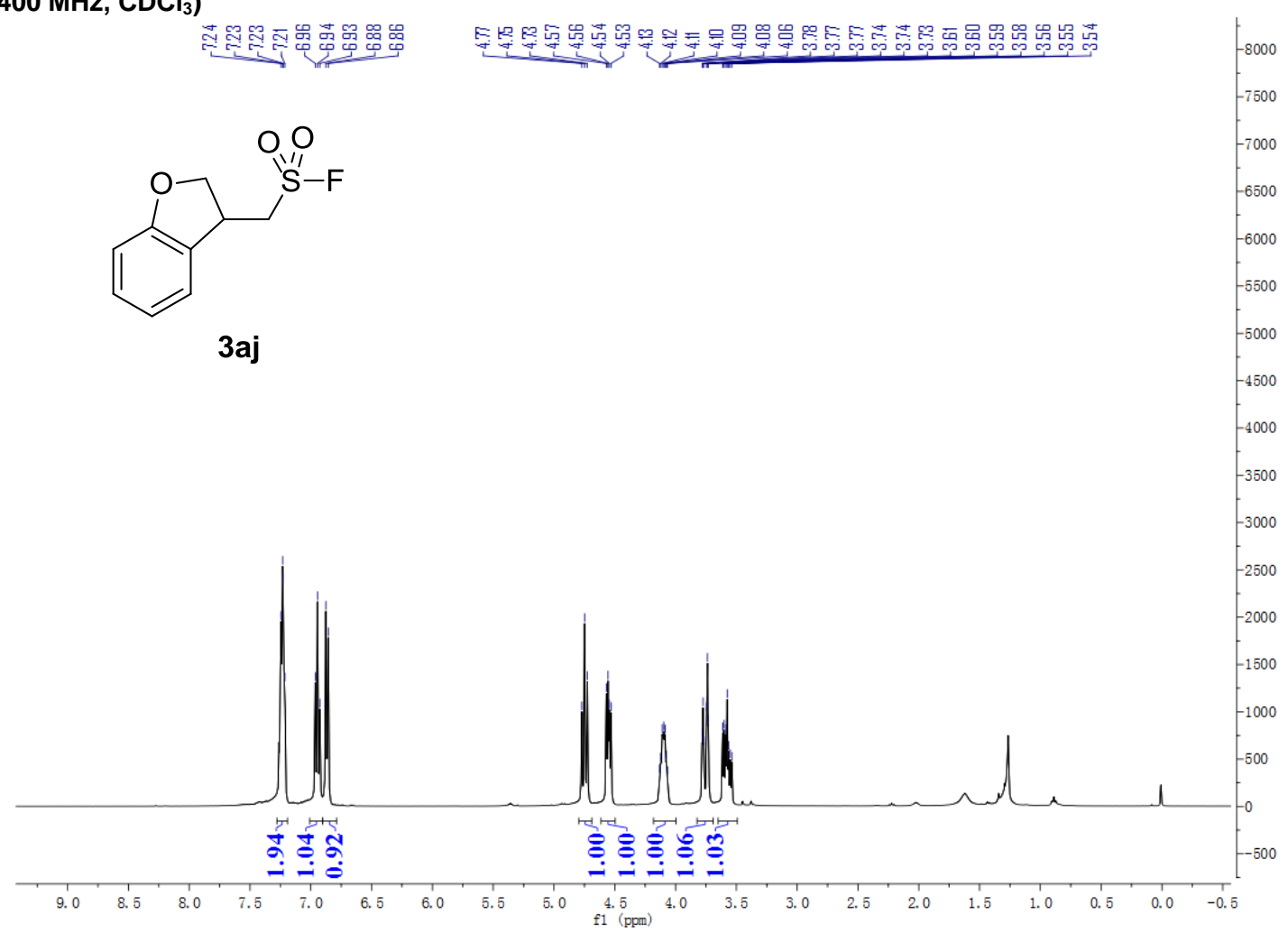




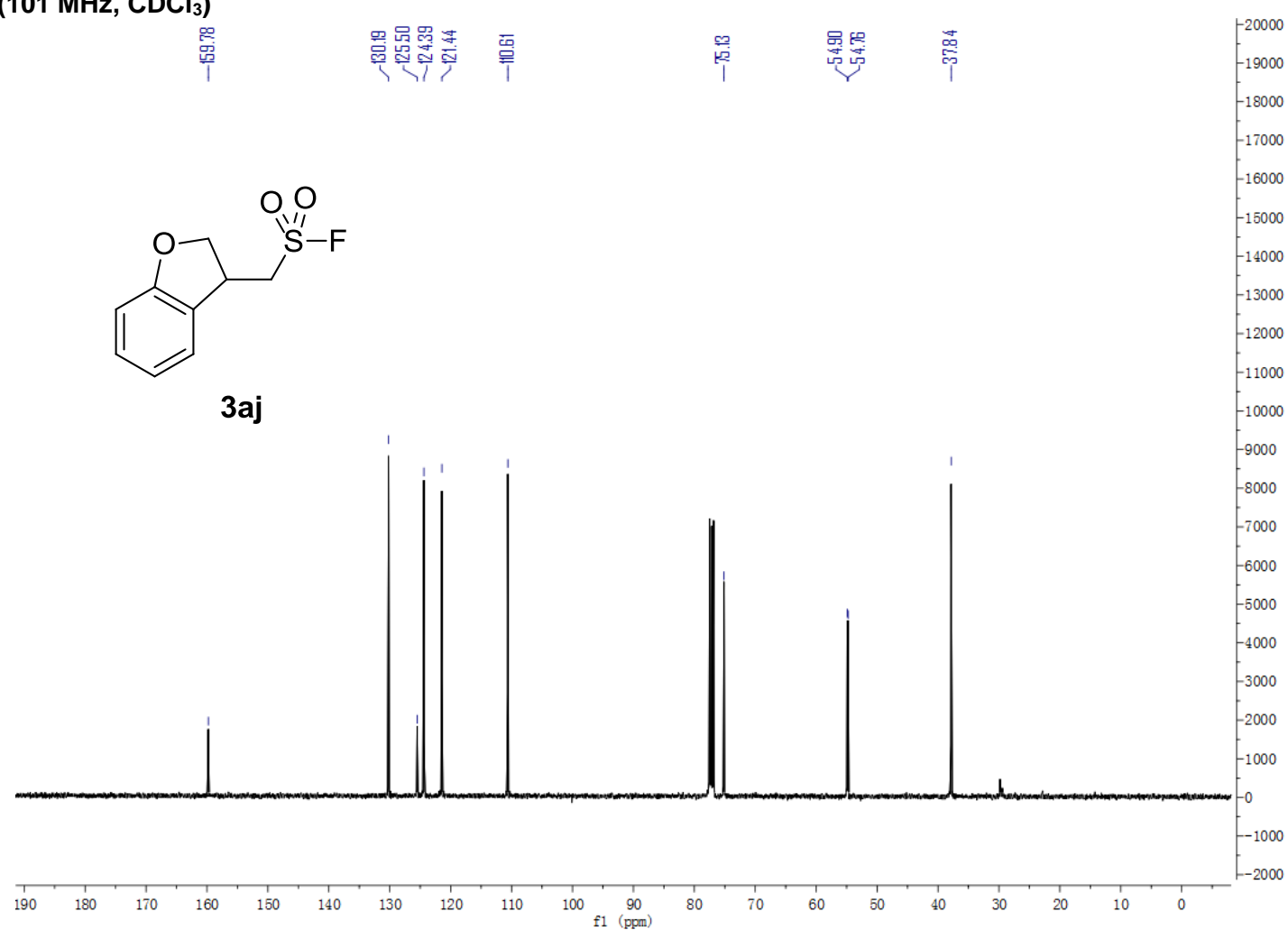

${ }^{19} \mathrm{~F} \mathrm{NMR} \mathrm{(376} \mathrm{MHz,} \mathrm{CDCl}_{3}$ )

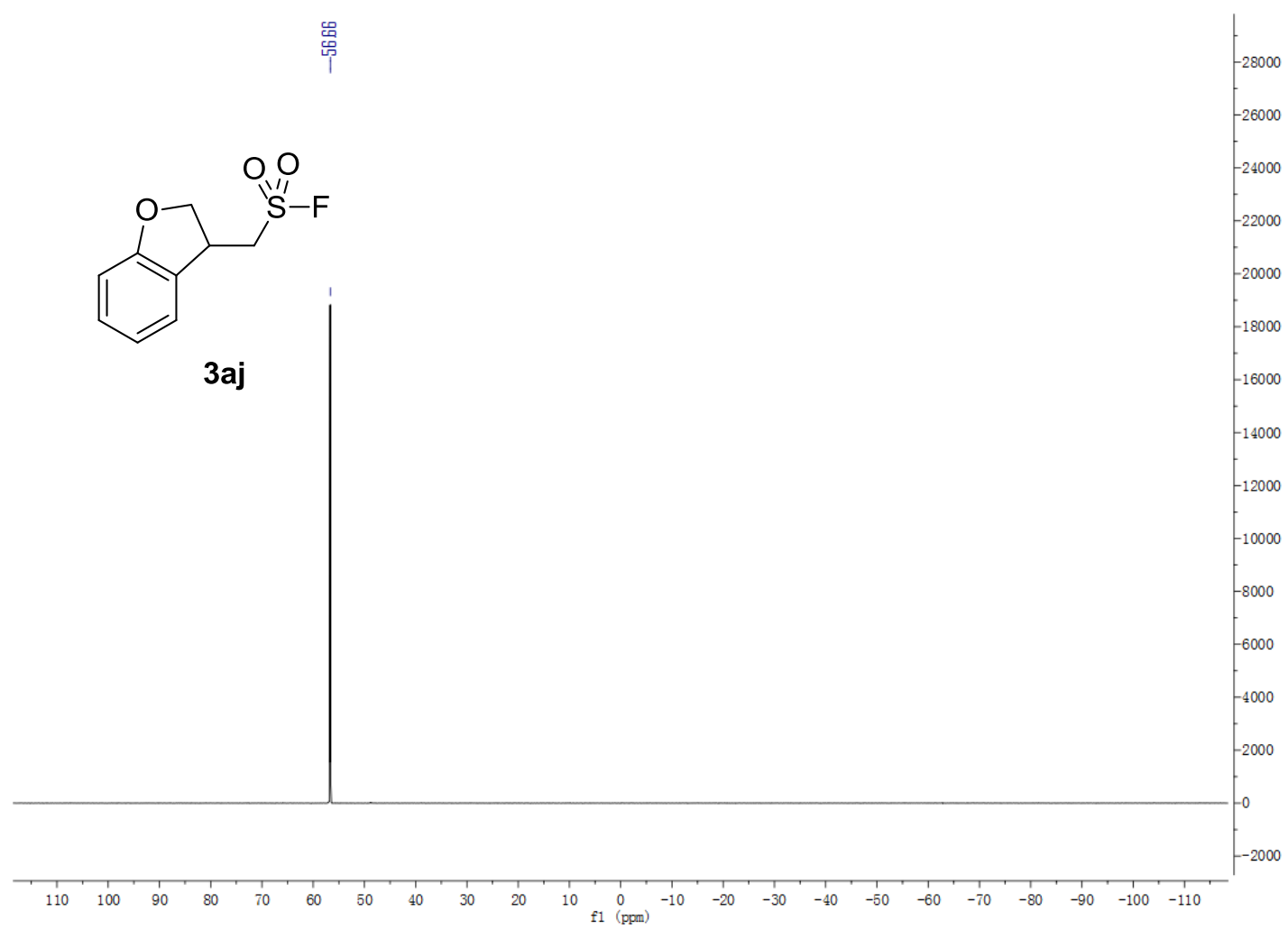


${ }^{1} \mathrm{H}$ NMR (400 MHz, $\mathrm{CDCl}_{3}$ )

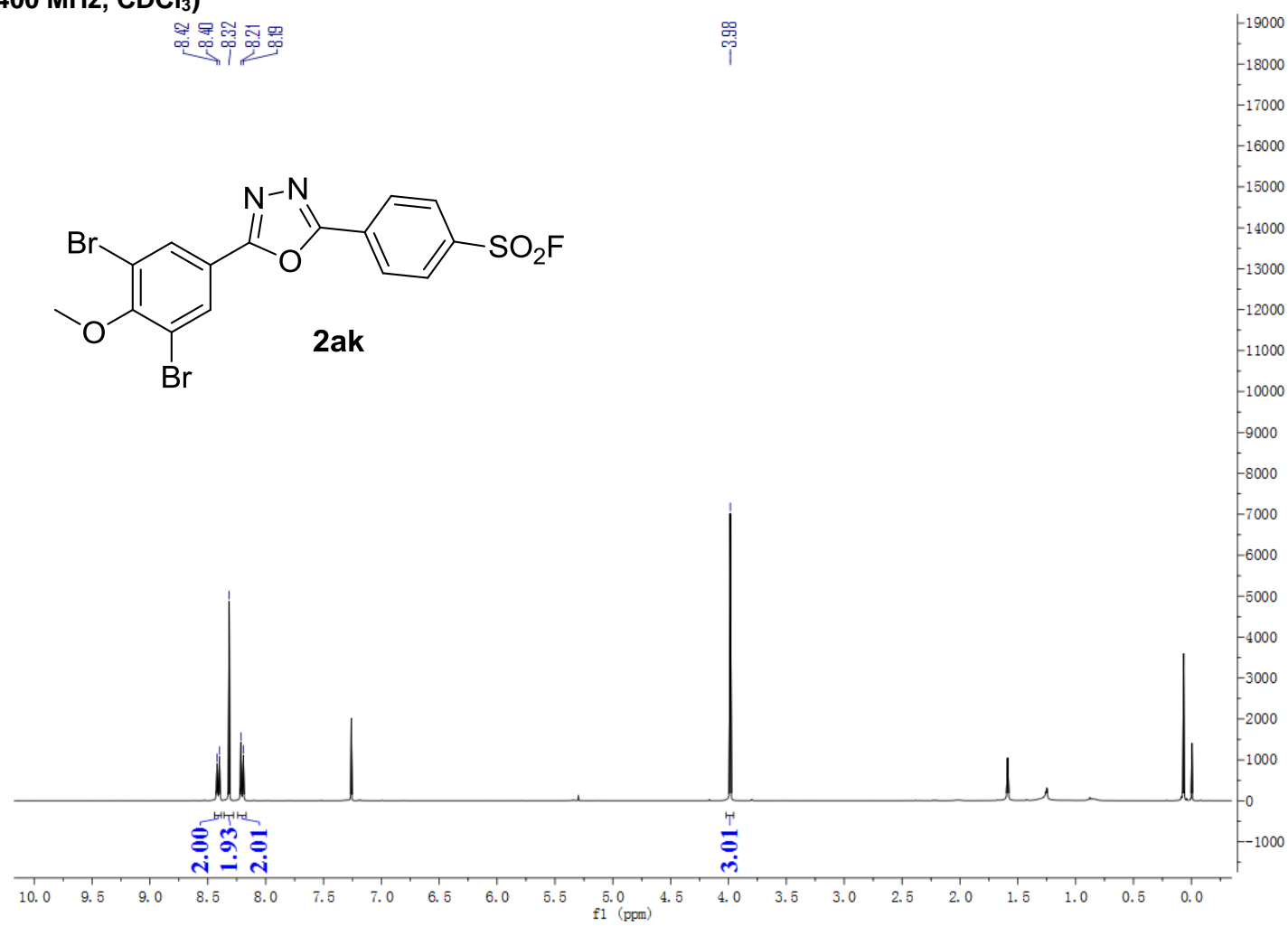

${ }^{13} \mathrm{C}$ NMR (101 MHz, $\mathrm{CDCl}_{3}$ )

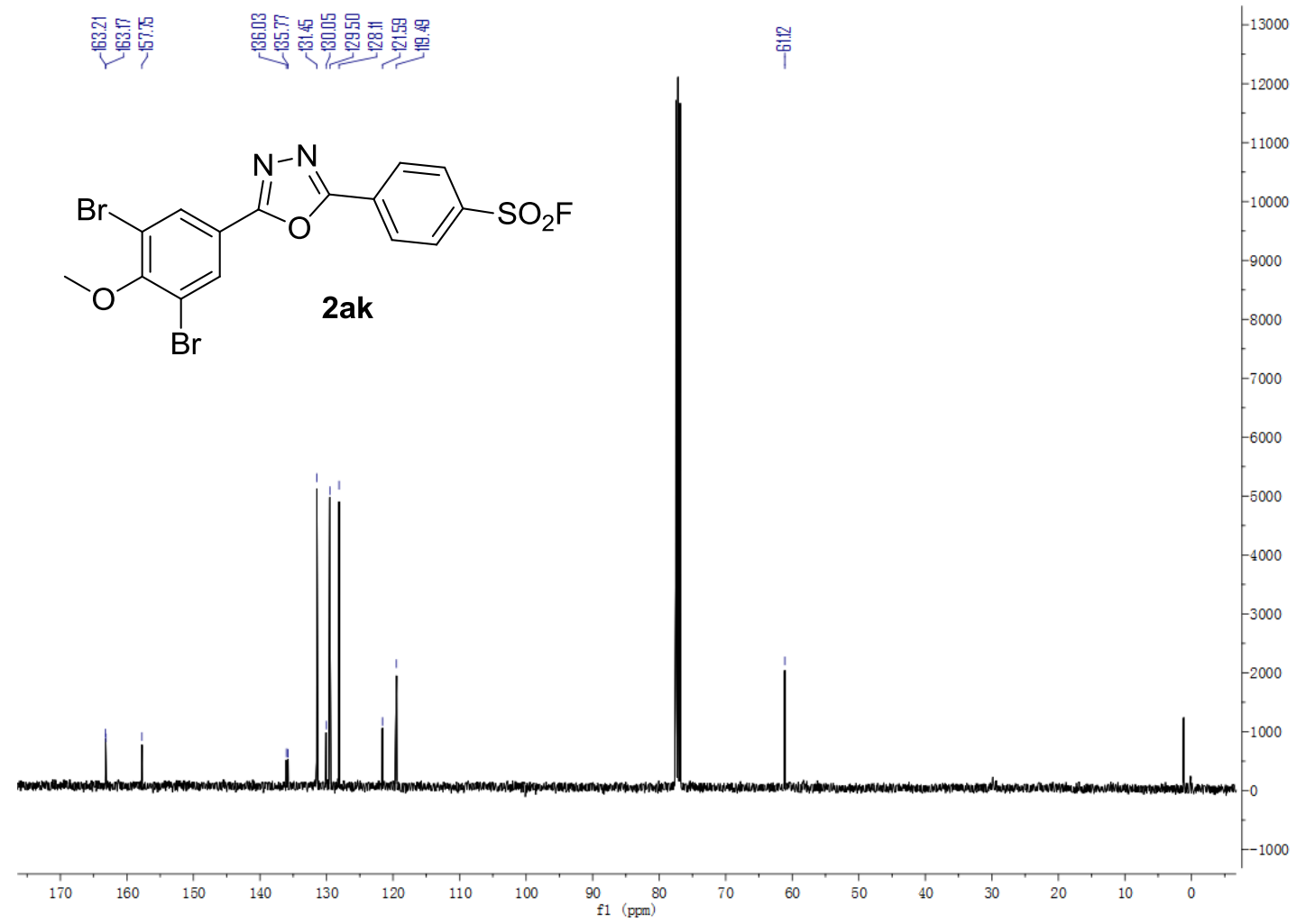


$\left.{ }^{19} \mathrm{~F} \mathrm{NMR} \mathrm{(376} \mathrm{MHz,} \mathrm{CDCl}_{3}\right)$

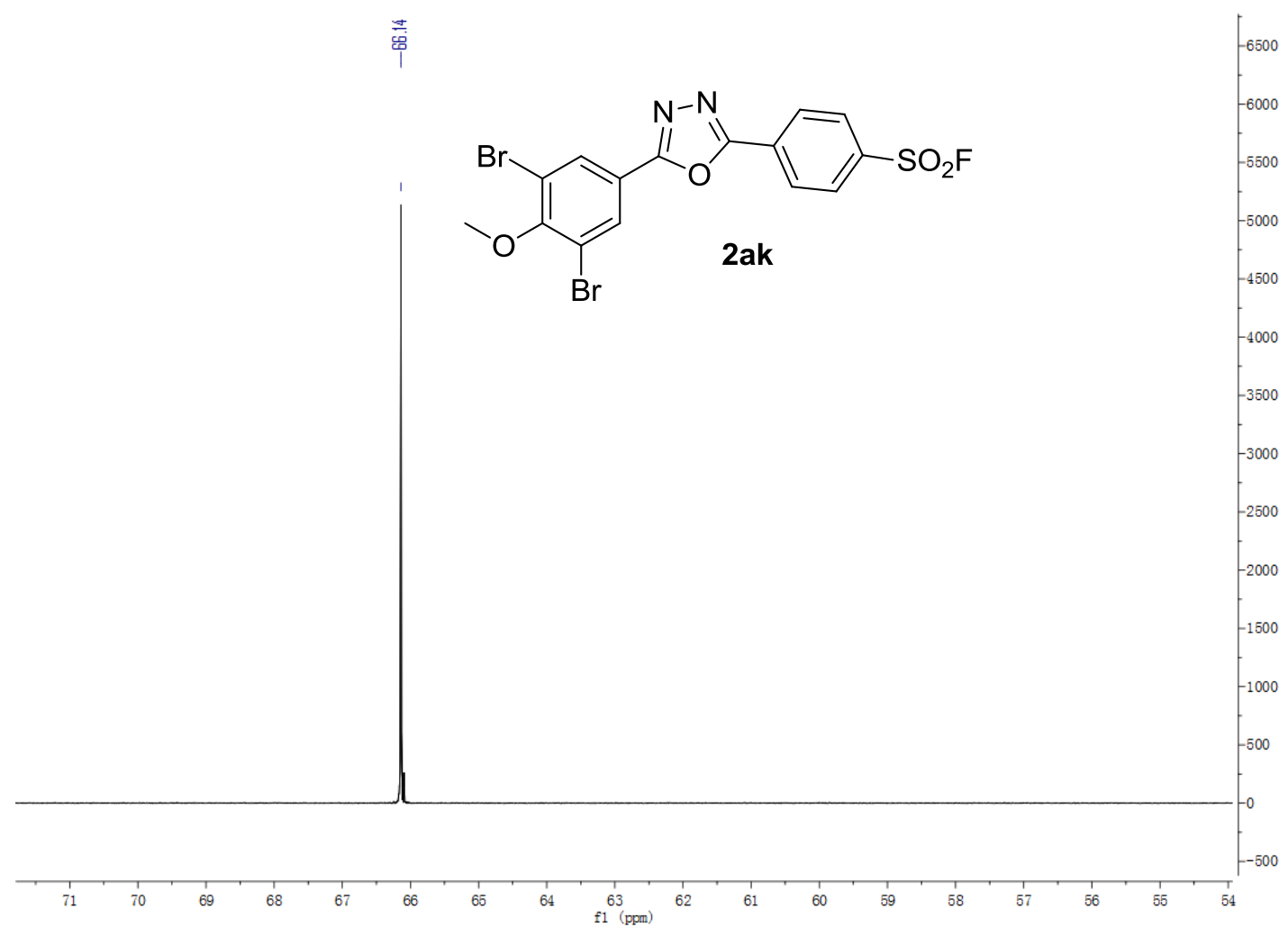


<smiles>Cc1ccc(S(=O)(=O)C=C(c2ccccc2)c2ccccc2)cc1</smiles>

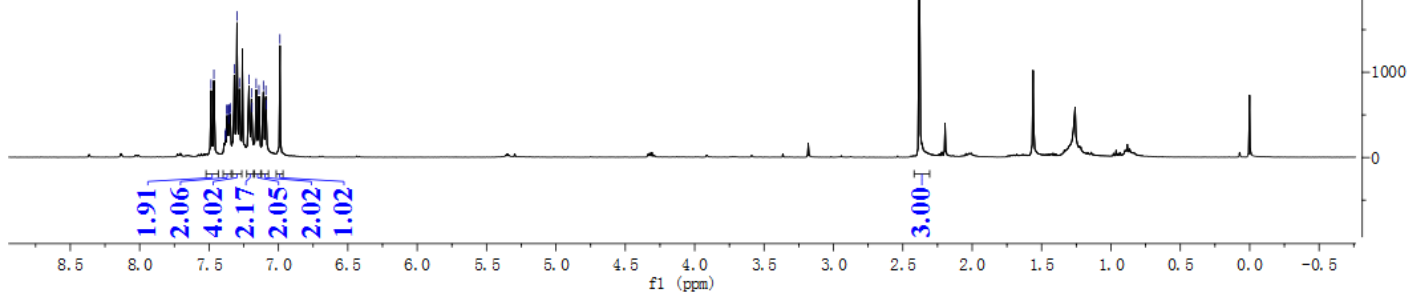

${ }^{13} \mathrm{C}$ NMR (101 MHz, $\mathrm{CDCl}_{3}$ )

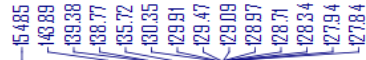

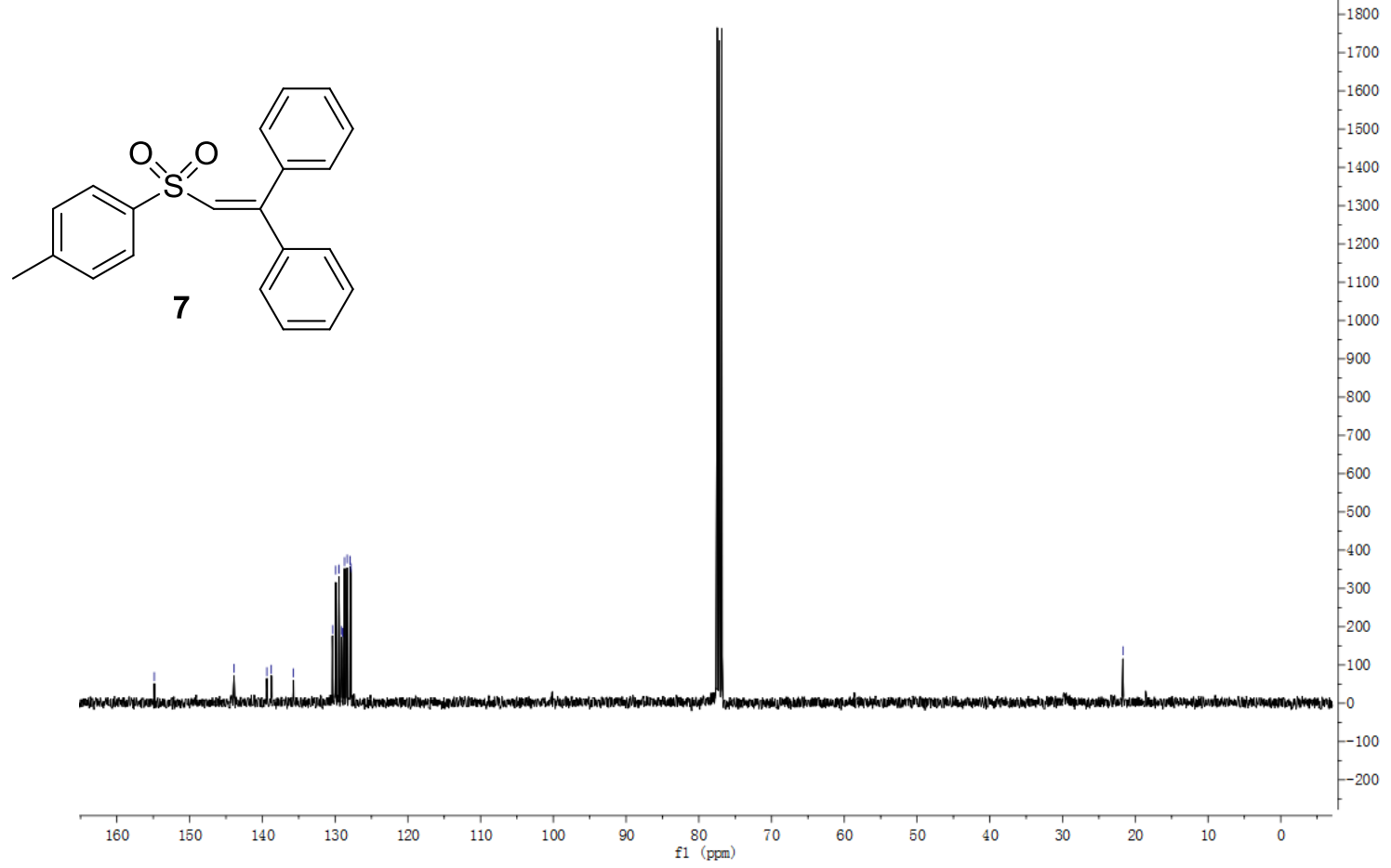


${ }^{1} \mathrm{H}$ NMR (400 MHz, $\left.\mathrm{CDCl}_{3}\right)$

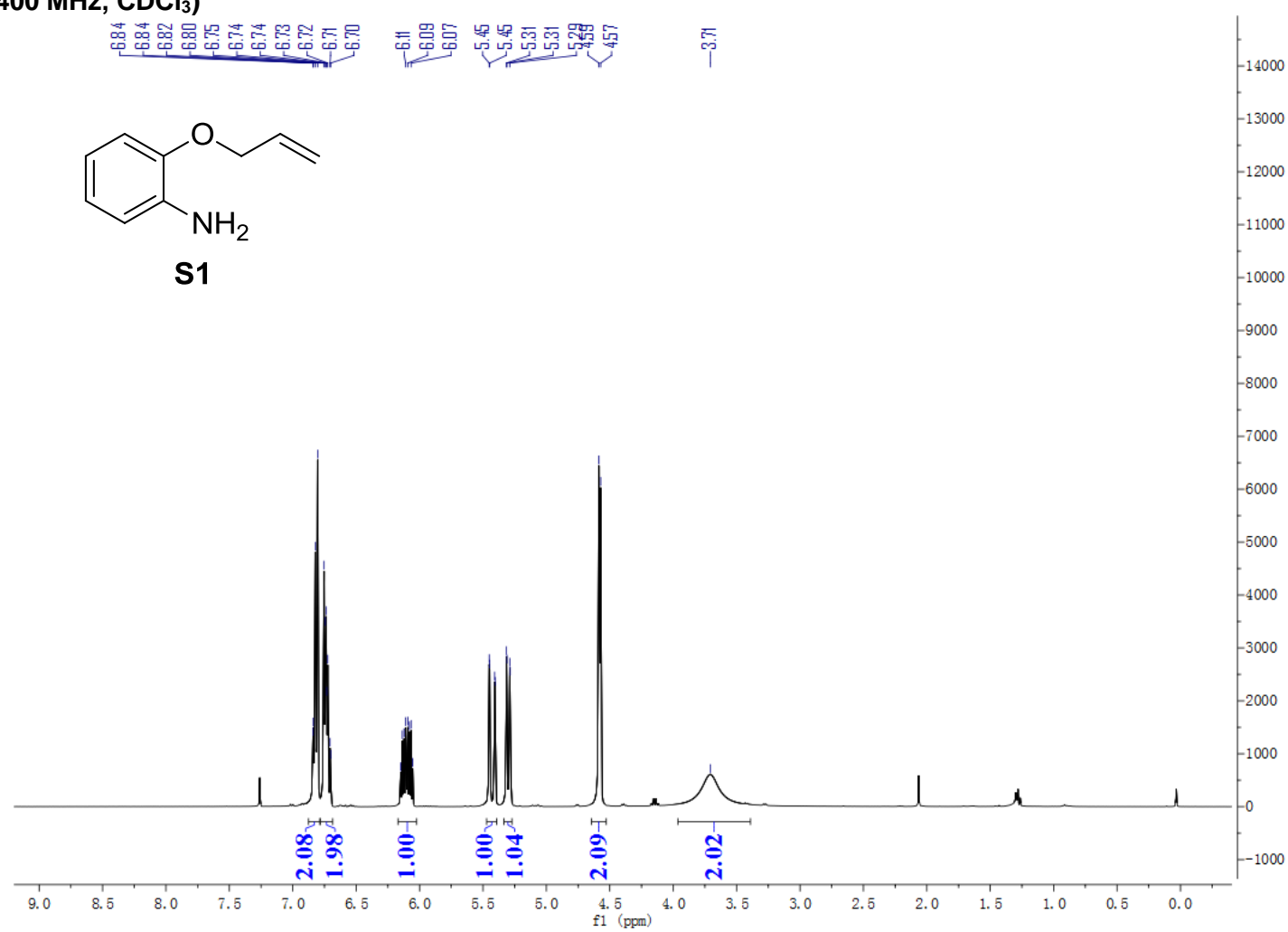

${ }^{13} \mathrm{C}$ NMR (126 MHz, $\mathrm{CDCl}_{3}$ )

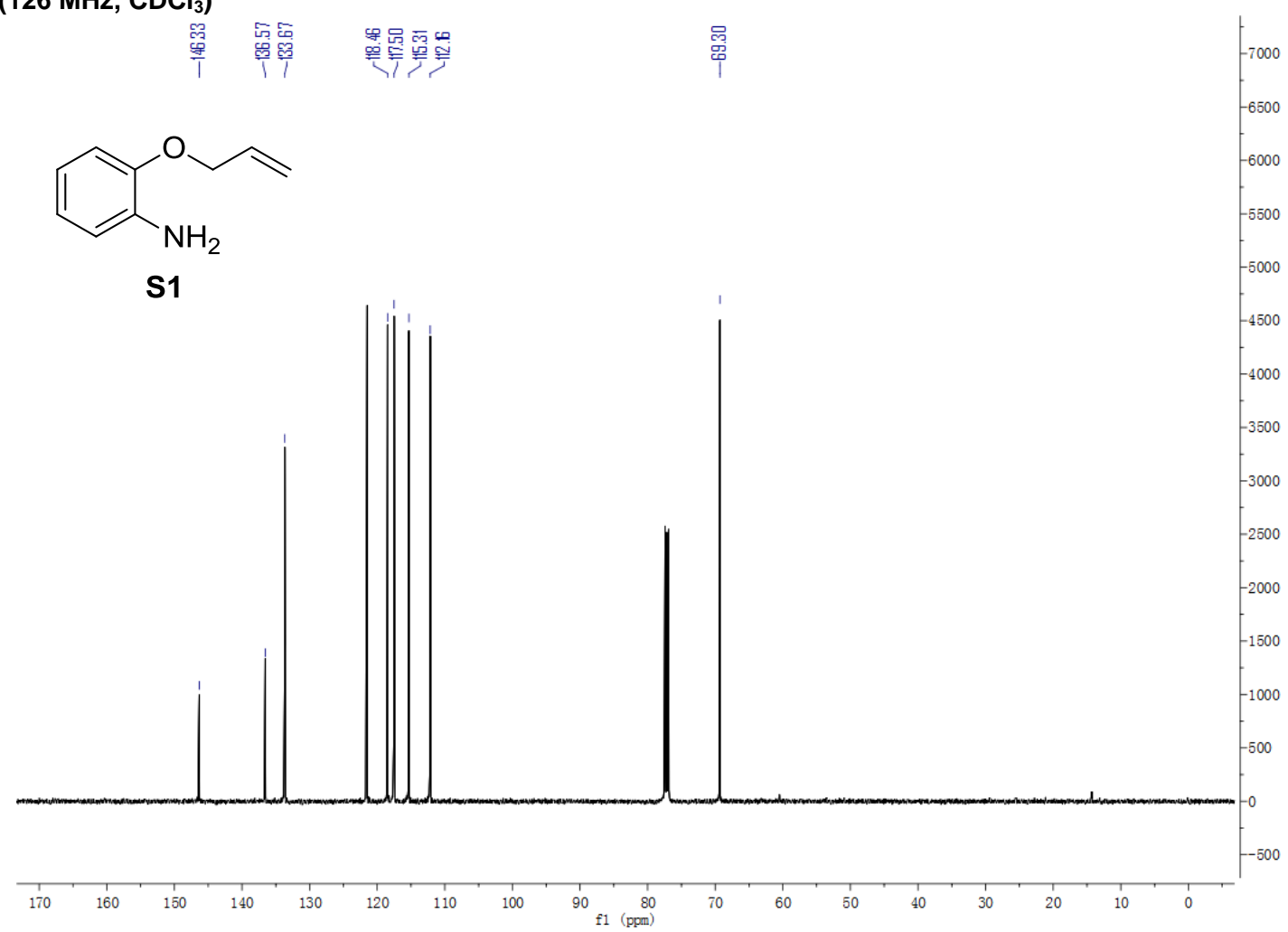


<smiles>CC(C)[C@H]1CC[C@@H](C)C[C@H]1OC(=O)c1ccc([N+](=O)[O-])cc1</smiles>

4
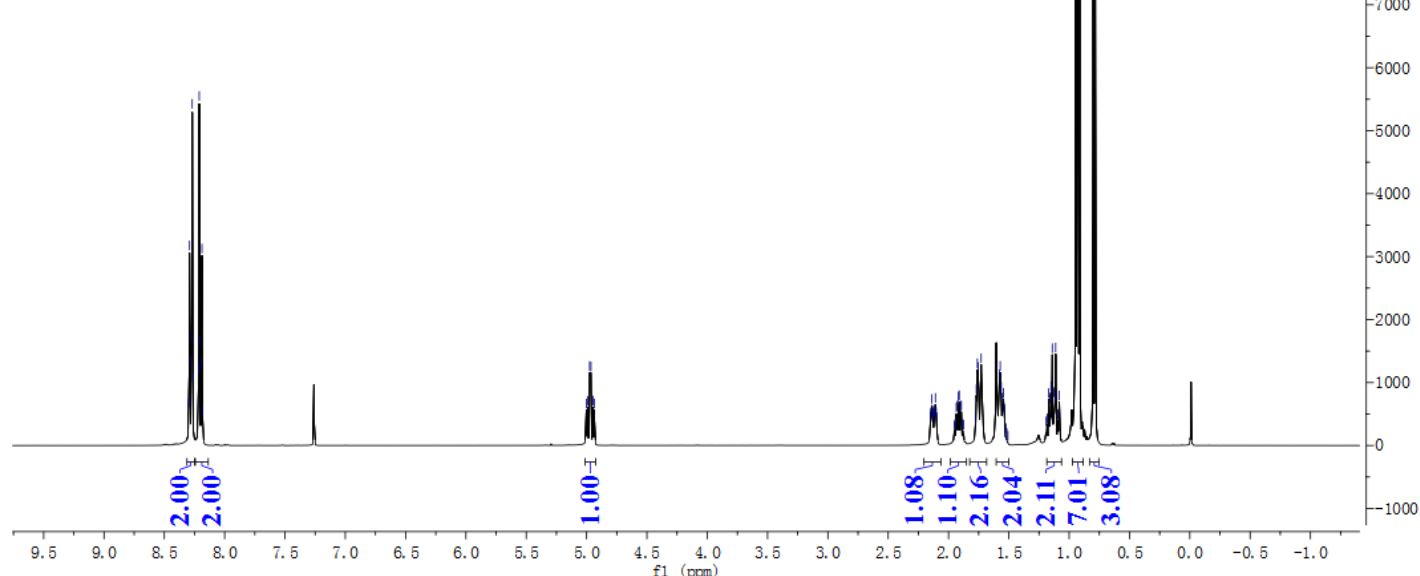

${ }^{13} \mathrm{C}$ NMR (101 MHz, $\mathrm{CDCl}_{3}$ )

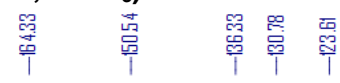

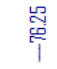

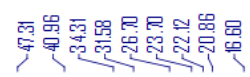

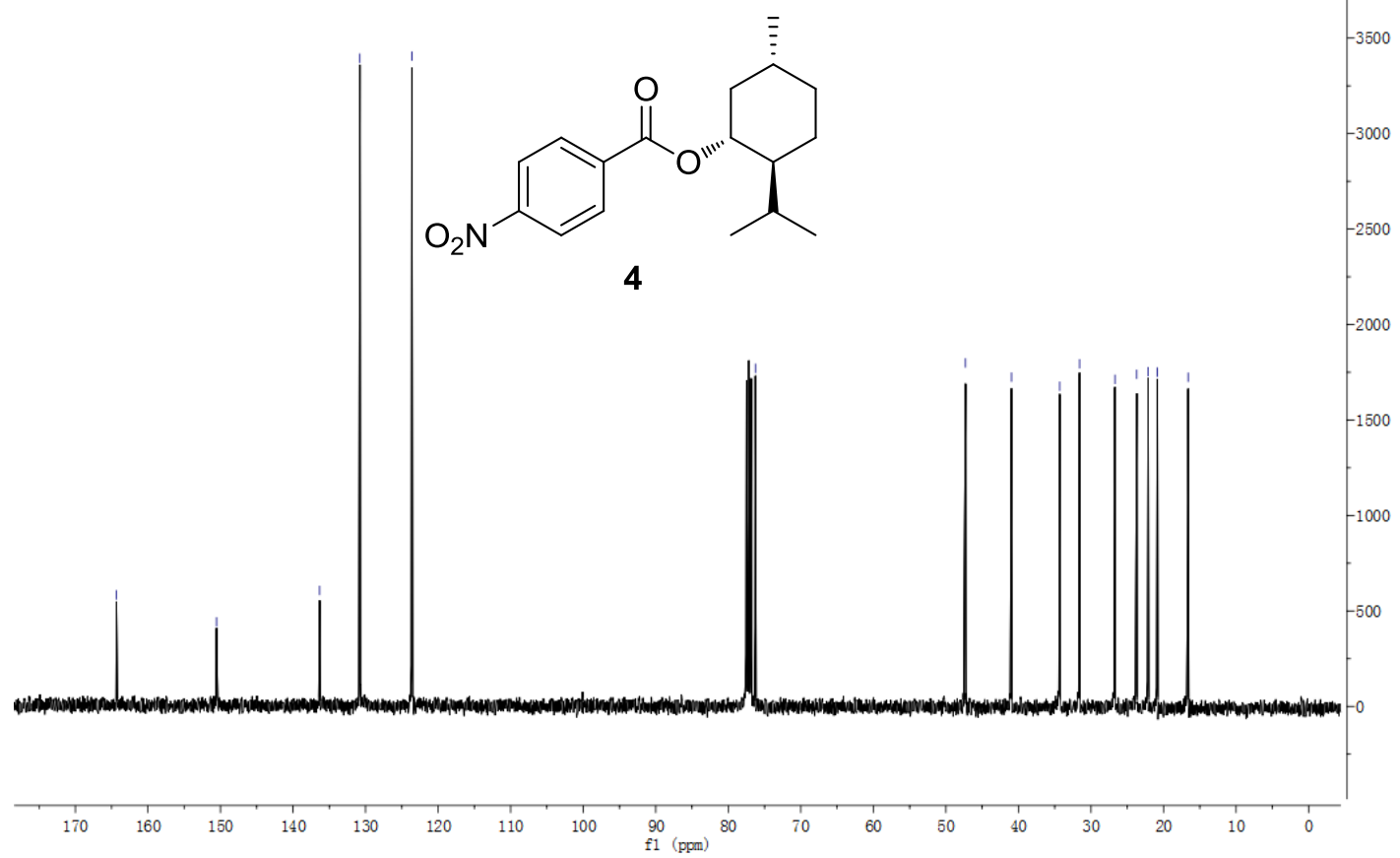




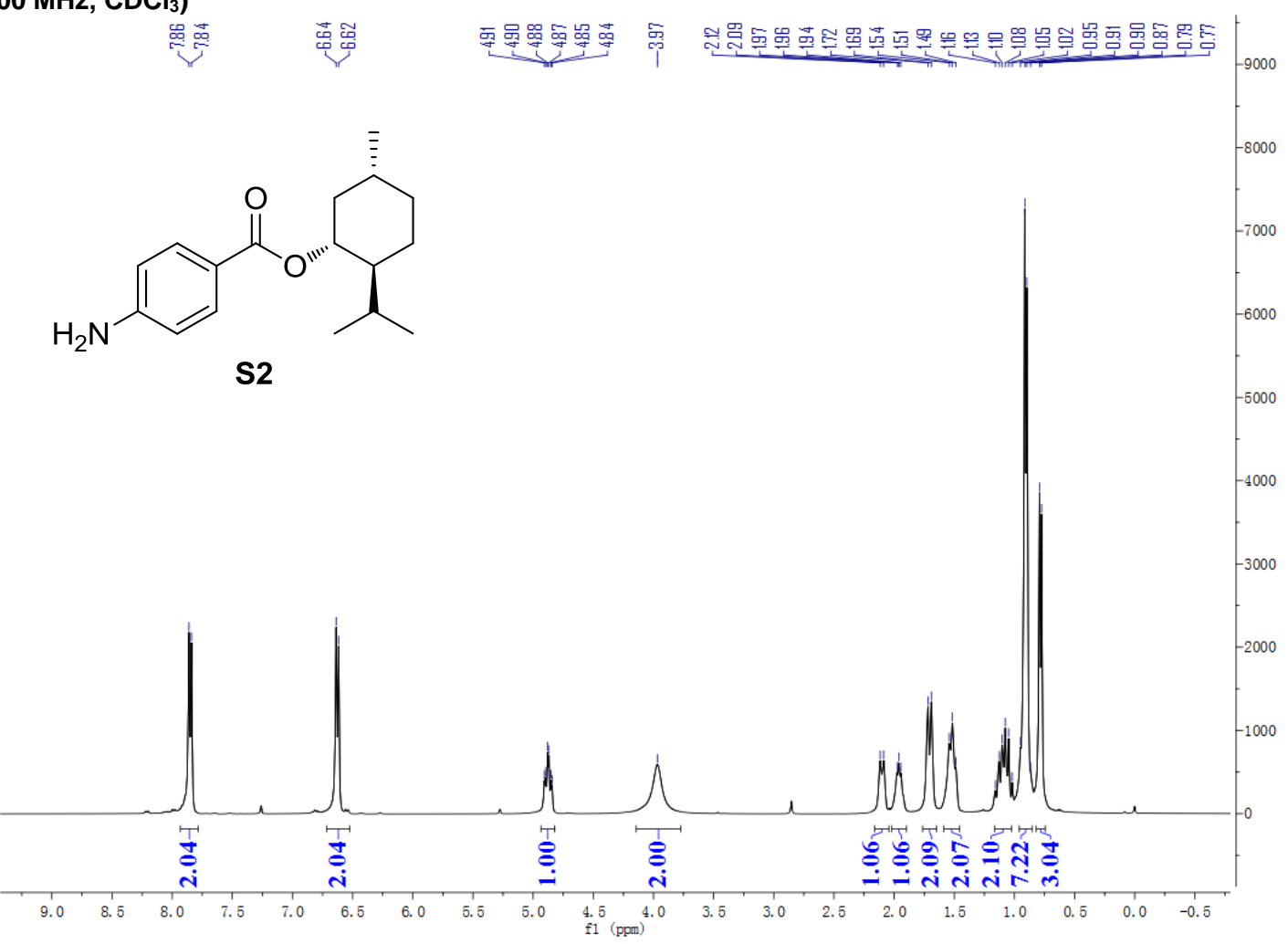

${ }^{13} \mathrm{C}$ NMR (101 MHz, $\mathrm{CDCl}_{3}$ )

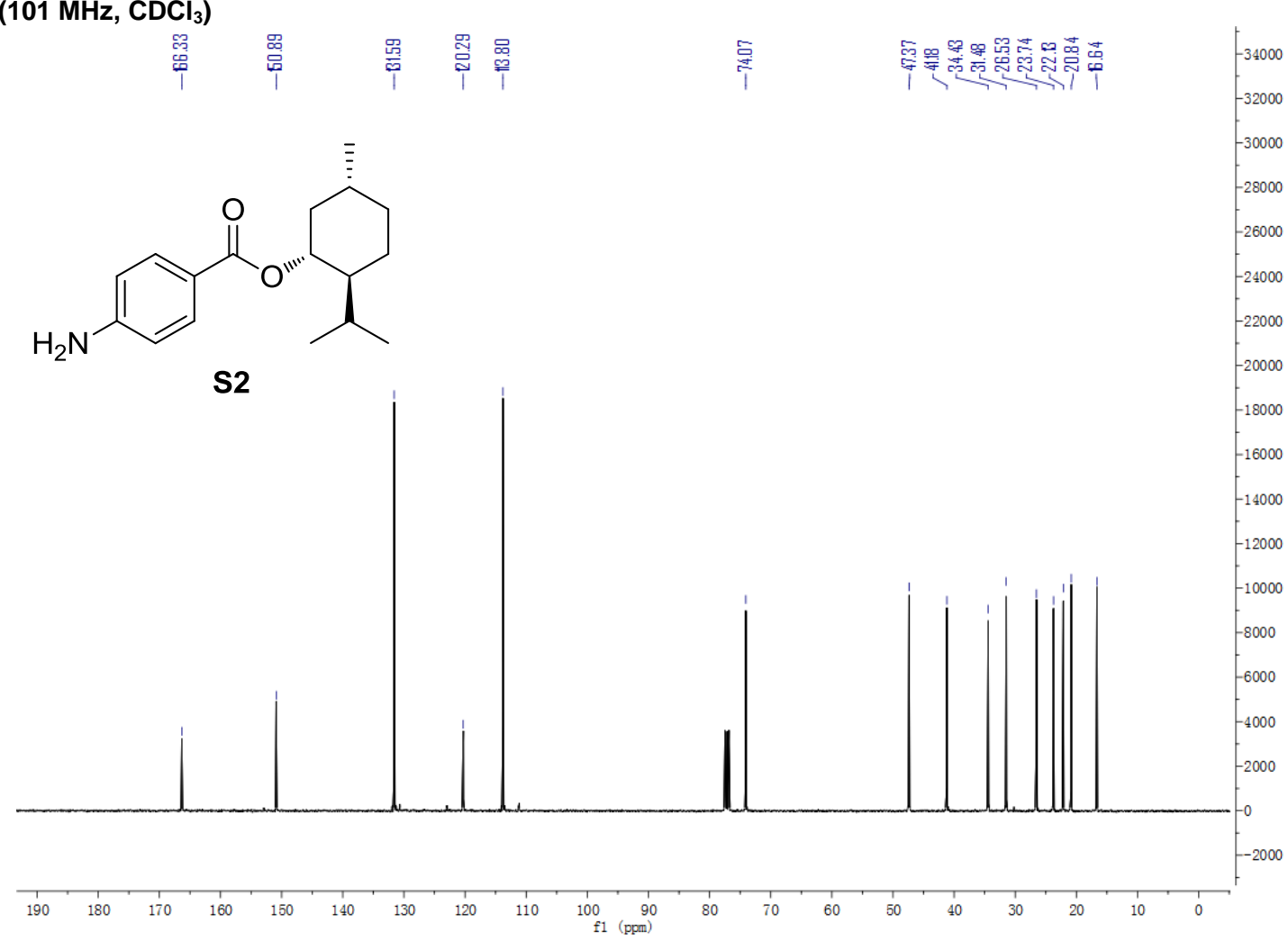




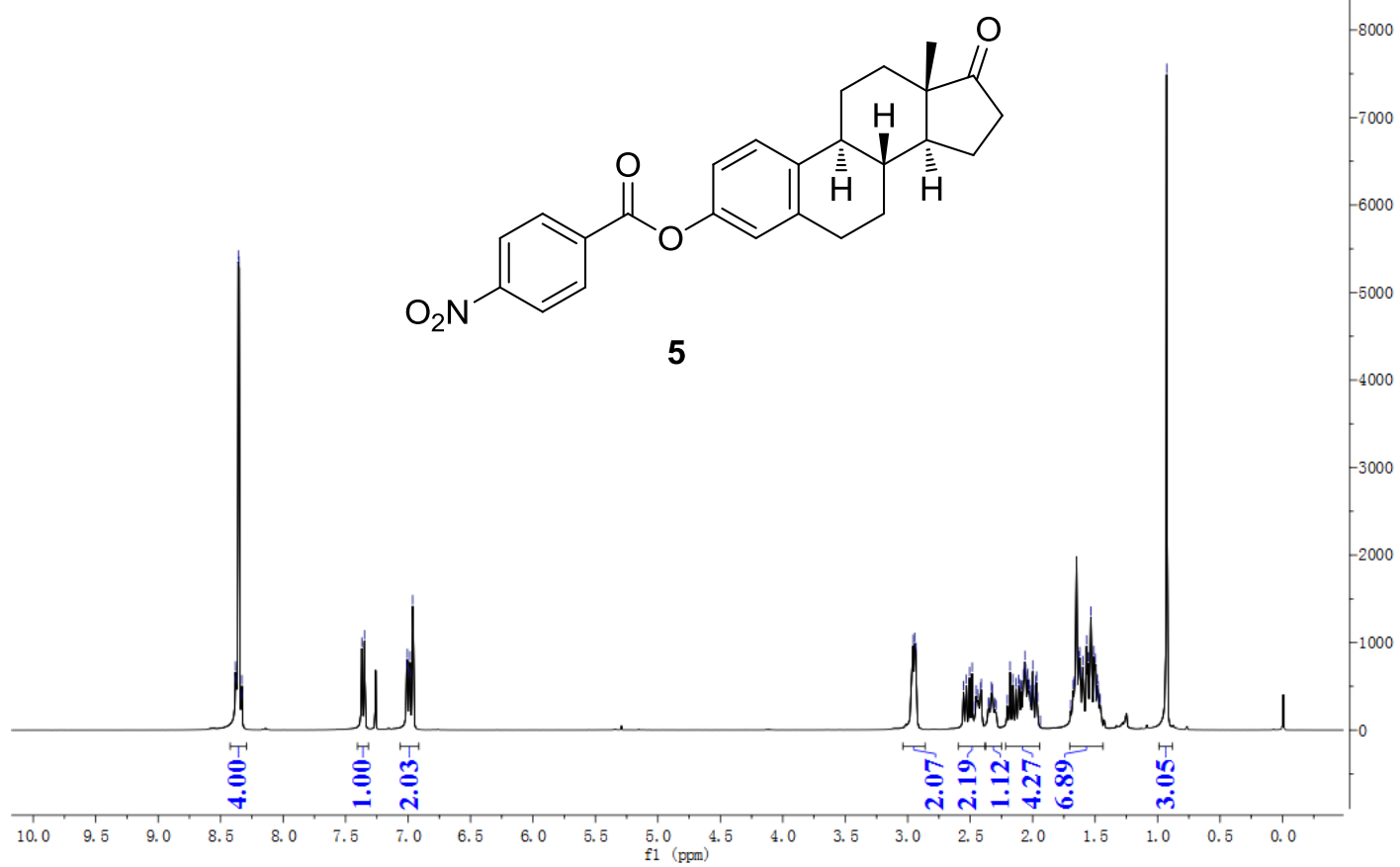

${ }^{13} \mathrm{C}$ NMR (101 $\left.\mathrm{MHz}, \mathrm{CDCl}_{3}\right)$

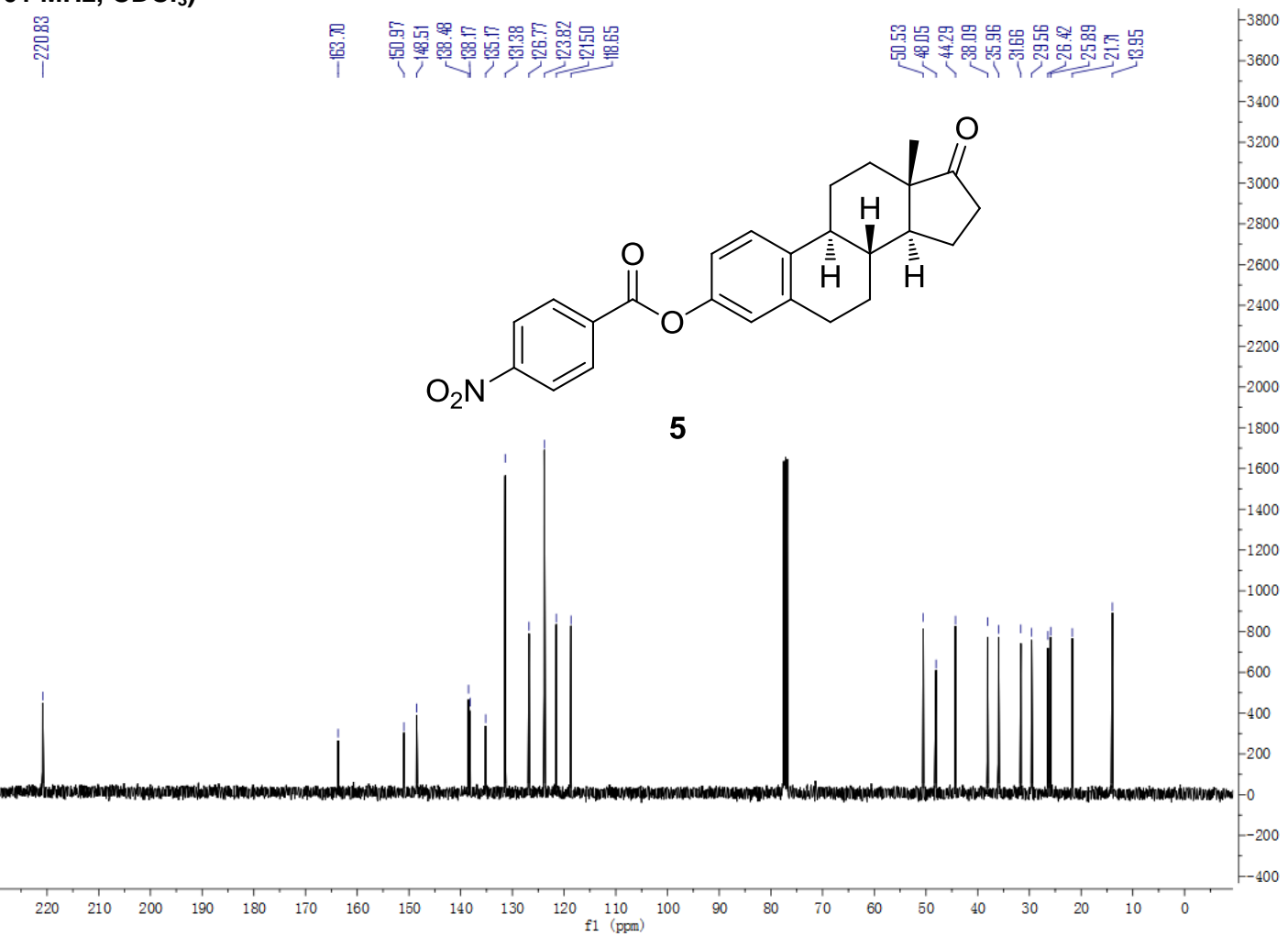


<smiles>C[C@]12CC[C@@H]3c4ccc(OC(=O)c5ccc(N)cc5)cc4CC[C@H]3[C@H]1CCC2=O</smiles>

S3
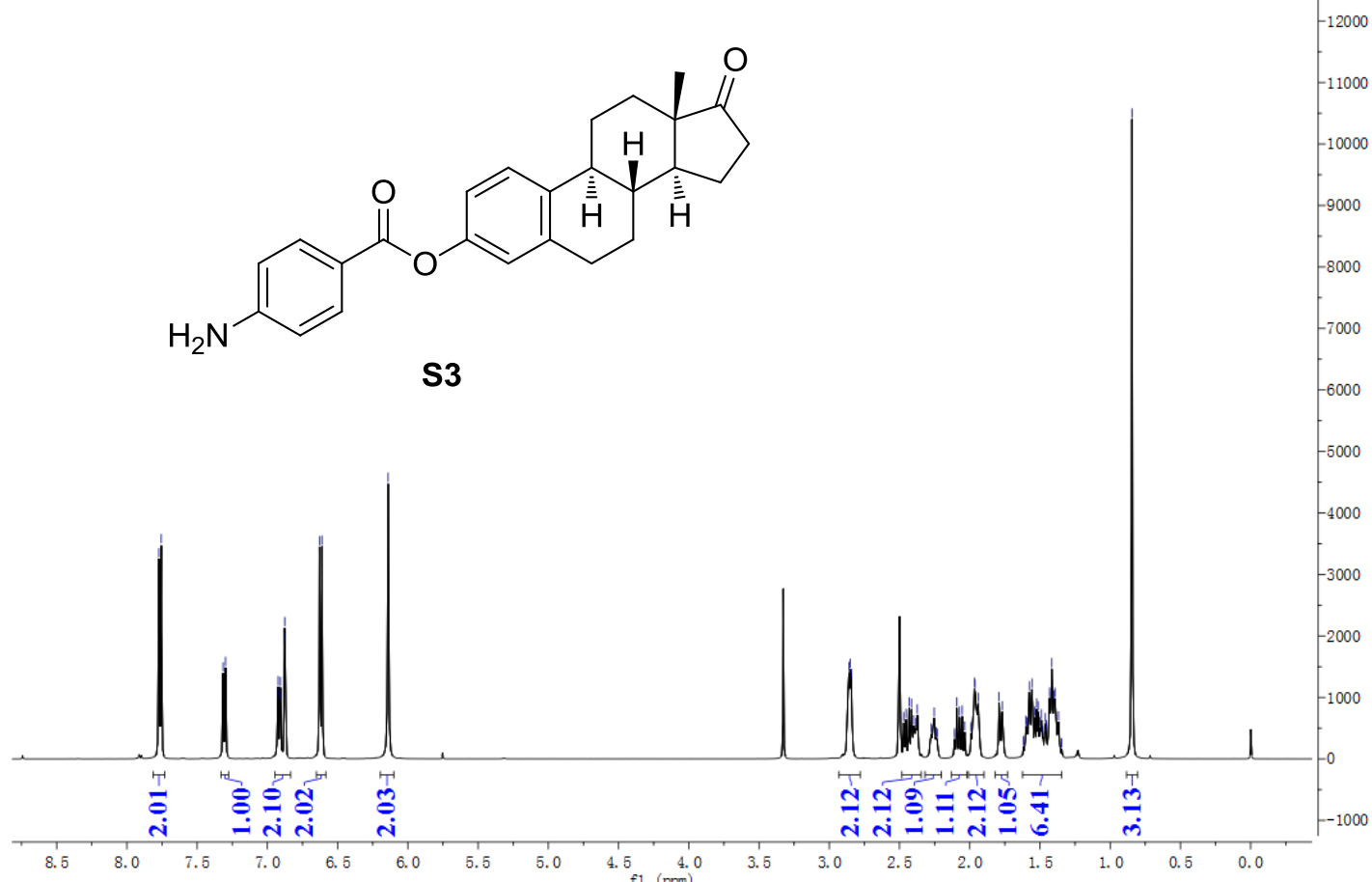

${ }^{13} \mathrm{C}$ NMR (101 MHz, DMSO-d 6 )

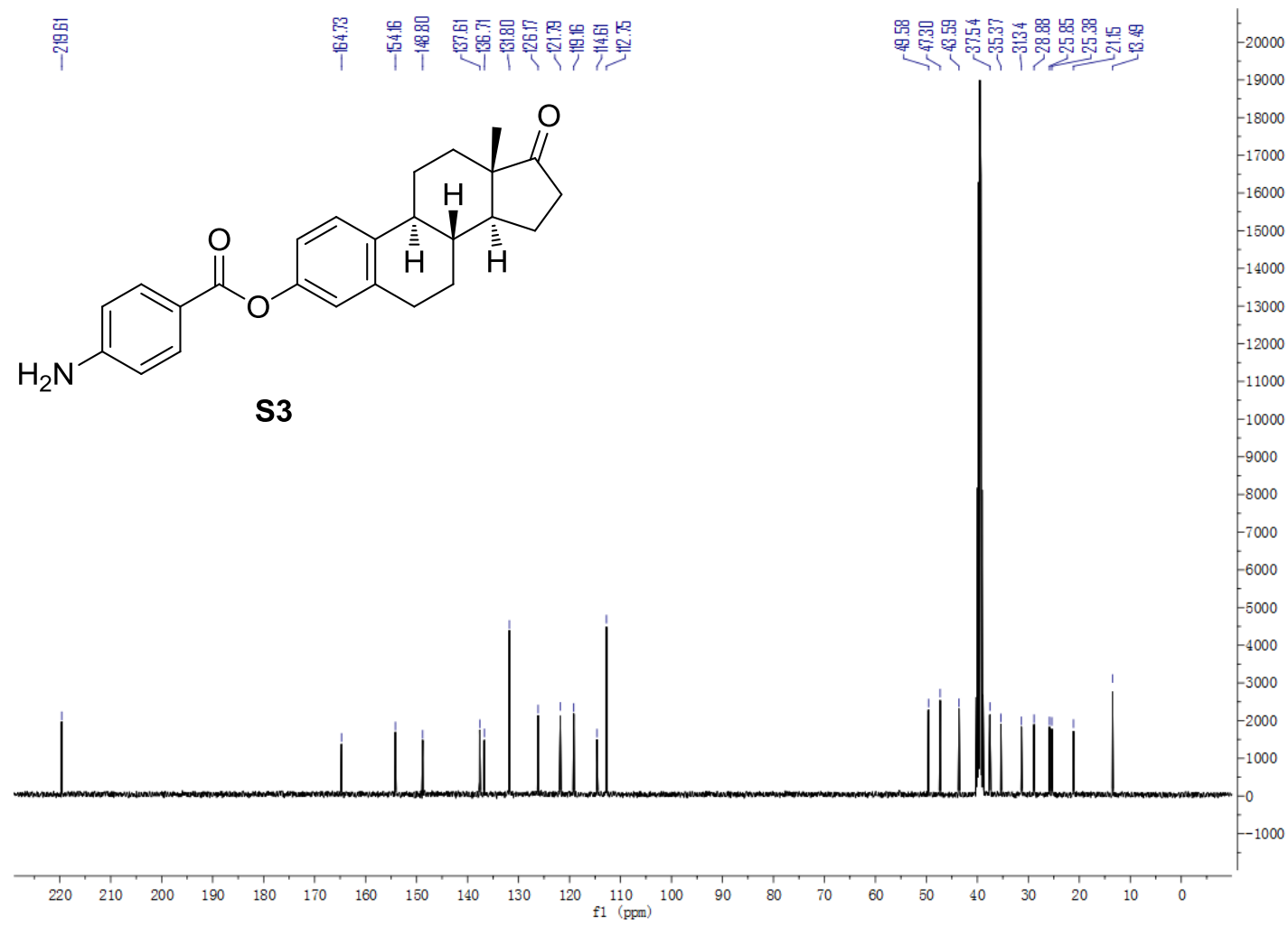


${ }^{1} \mathrm{H}$ NMR (400 MHz, DMSO-d ) $^{2}$

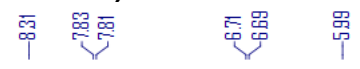

㗊<smiles>COc1c(Br)cc(-c2nnc(-c3ccc(N)cc3)o2)cc1Br</smiles>

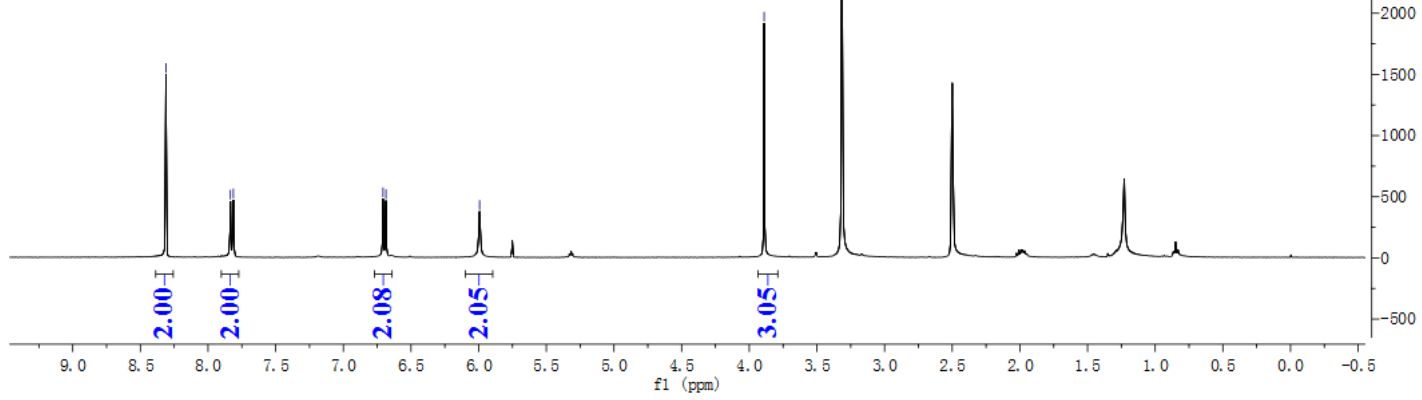

${ }^{13} \mathrm{C}$ NMR (126 MHz, DMSO-d 6 )

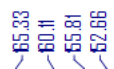

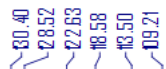<smiles>COc1c(Br)cc(-c2nnc(-c3ccc(N)cc3)o2)cc1Br</smiles>

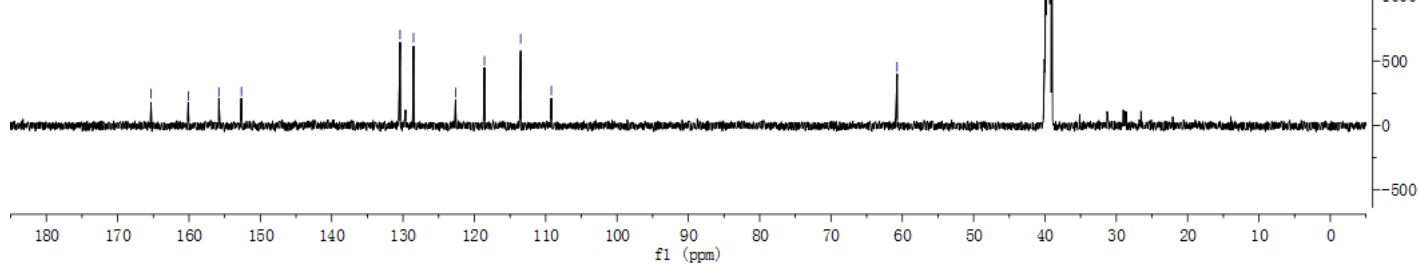

